\title{
RADIATORS FOR AIRCRAFT ENGINES
}

\author{
By S. R. Parsons and D. R. Harper 3d
}

\section{ABSTRACT}

The characteristics which determine the value of the radiator in discharging its functions are considered in detail. Measurements of air flow through the core, of head resistance, of cooling power, and of geometrical characteristics are described and an exposition given of the relations between these and the conditions under which a radiator operates and its characteristics of form and construction. The work was based on special laboratory investigations, including laboratory tests of over roo types of radiator core. A detailed record of the performance of these cores is included in the paper.

\section{CONTENTS}

I. Introduction

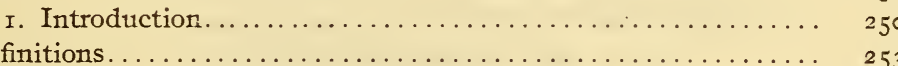

2. Conditions under which the radiator works.......... 253

3. Characteristics that identify the radiator core.......... 253

4. Properties that define the performance of a radiator........ 255

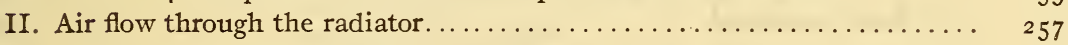

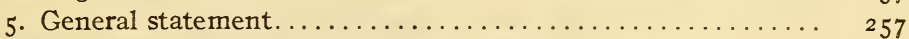

A. Experimental method of measuring air flow and computation.... $\quad 259$

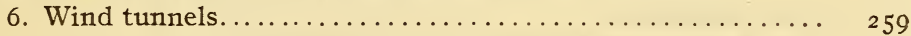

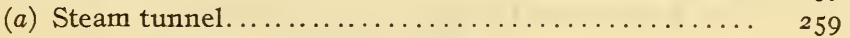

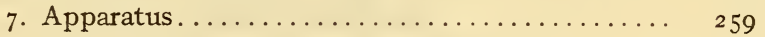

8. Calibrations....................... ${ }_{26} 60$

9. Observations.......................... 262

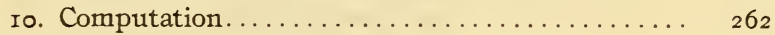

(b) Reduced pressure tunnel.................. ${ }_{2} 64$

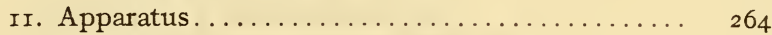

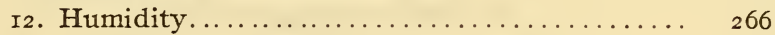

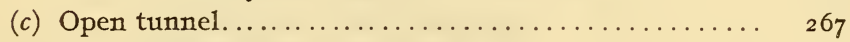

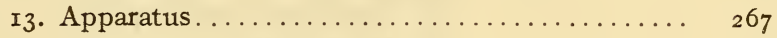

I4. Method of measurement............. ${ }_{2} 67$

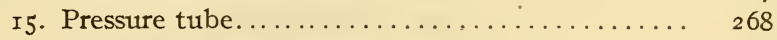

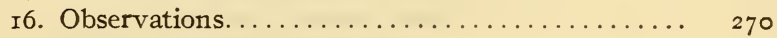

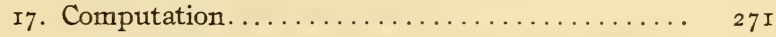

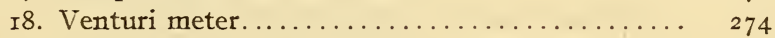

B. Air flow for unobstructed positions of radiator. . . . . . . . 275

I9. Speed and density.................. 275

20. Empirical equation for air flow constant...... 276

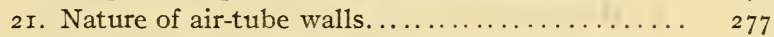

22. Yaw............................ 278 
II. Air flow through the radiator-Continued

C. Air flow for obstructed and slip-stream positions. .............

23. Nose of the fusela

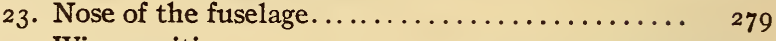

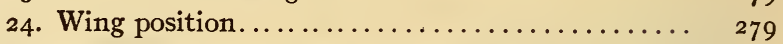

25. Slip-stream positions. ................... 280

III. Head resistance and power absorbed...................... $28 \mathrm{r}$

26. General statement. ..................... $28 \mathrm{r}$

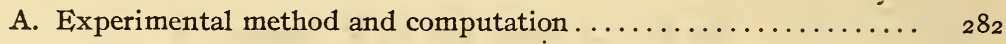

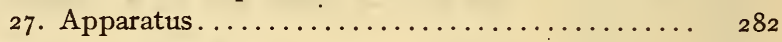

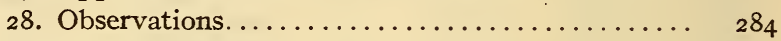

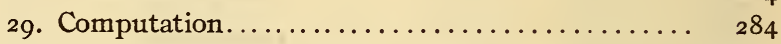

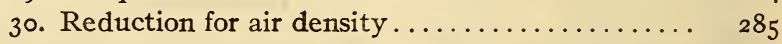

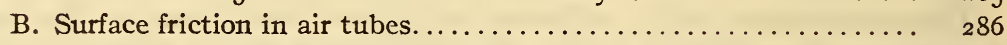

3r. Radiators not similar to long tubes......... 286

32. Comparison of pressure gradients in radiators with values for long tubes............. 287

33. Condition of surface................ 288

34. Effect of heat in the radiator............ 290

C. Head resistance of the core ......................... 29 I

35. Flying speed and air density............ $29 \mathrm{I}$

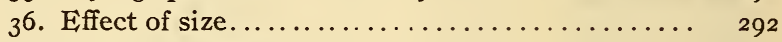

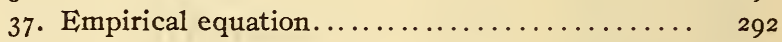

38. Effects of design of core................ 293

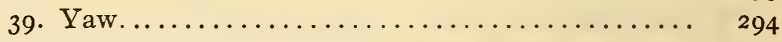

D. Head resistance chargeable to the radiator................. 294

40. Unobstructed positions............... 294

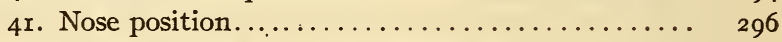

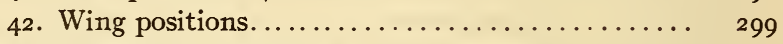

43. Slip-stream positions................. 300

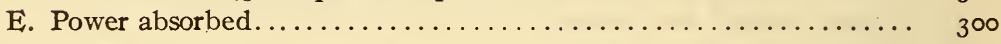

44. Power absorbed by the core............ 300

45. Power absorbed chargeable to the radiator..... 302

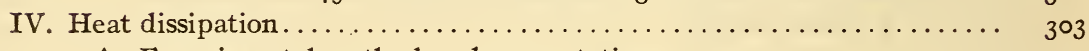

A. Experimental method and computation ............... $3 \circ 3$

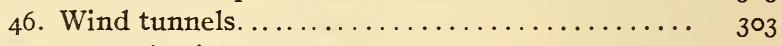

(a) Steam tunnel using steam.................... $3 \circ 3$

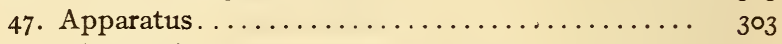

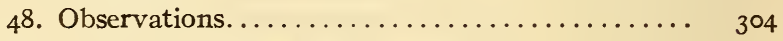

49. Computation of air flow............... 306

50. Computation of heat transfer............. 306

(b) Reduced-pressure tunnel.................. 308

51. Apparatus-air system.............. 308

52. Apparatus-water system............. 309

53. Observations............................. 3 I0

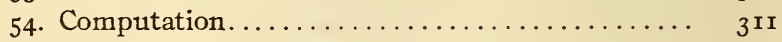

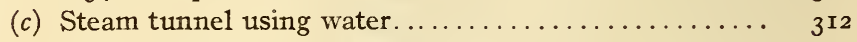

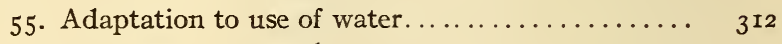

B. Heat transfer, water or steam to metal....................... $3^{\text {I2 }}$

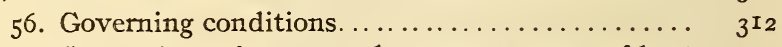

57. Comparison of steam and water as sources of heat

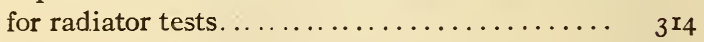

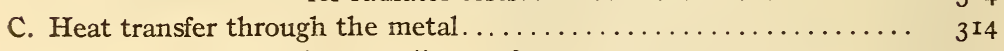

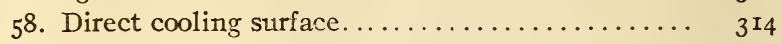

59. Indirect cooling surface............ $3^{15}$ 
IV. Heat dissipation-Continued

D. Heat transfer, metal to air $\ldots \ldots \ldots \ldots \ldots \ldots \ldots$

6o. Temperature difference.................. $3{ }_{3}{ }_{5}$

6r. Mass flow of air...................... 316

62. Empirical equation for variation of heat dissipation with air flow......................

63. Equation for variation of heat dissipation with

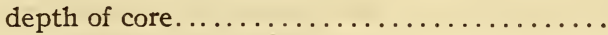

64. Relation between heat dissipation and surface friction........................ 320

65. Amount of cooling surface.............. 322

66 . Condition of cooling surface............ 323

67 . Indirect cooling surface.............. 327

68. Effectiveness of indirect cooling surface...... 328

69. Computation of heat dissipation for surface partly direct and partly indirect......................... 328

V. Effects of geometrical characteristics on performance............ 330

A. Effects of features of core design on performance........... 330

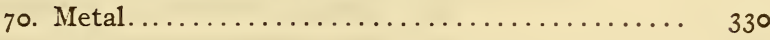

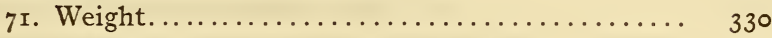

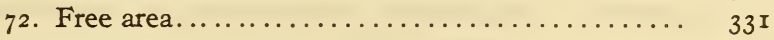

73. Amount of cooling surface................... 33 I

74. Condition of cooling surface............ 33 I

75. Proportions of fins for given effectiveness...... 332

76. Depth of radiator.................. 333

77. Form and dimensions of water passages....... 339

78. Form and dimensions of air passages........ 339

B. Special types of radiators. ....................... 34 I

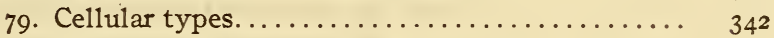

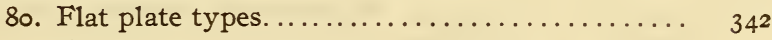

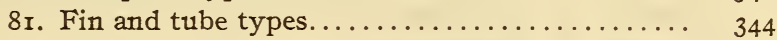

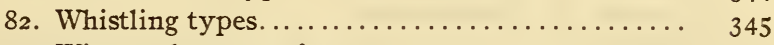

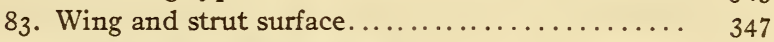

84. Stream-line casing for radiator........................ 348

C. Methods of measuring geometrical characteristics.......... 350

85. Measuring dimensions of the water tubes....... 350

86. Measuring dimensions of the air tubes........ $35^{\mathrm{I}}$

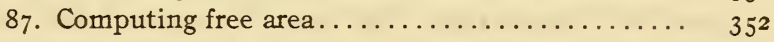

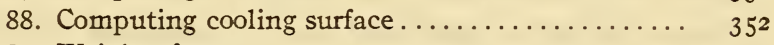

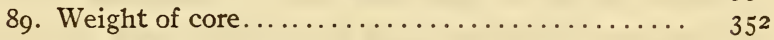

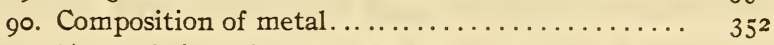

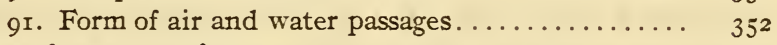

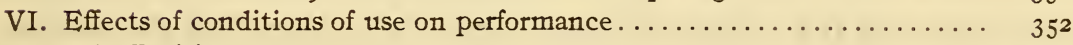

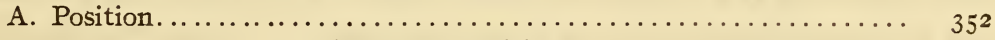

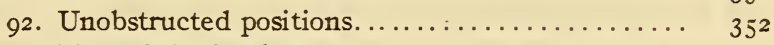

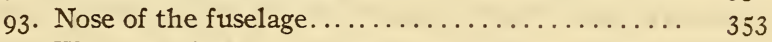

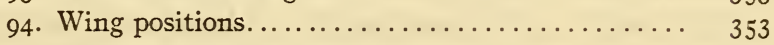

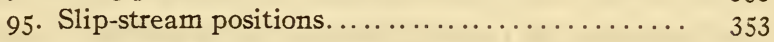

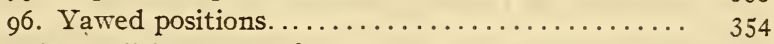

B. Effects of altitude conditions on performance............ 354

97. Atmospheric conditions at altitudes........ 354

98. Equations of performance.............. 356

99. Effects of altitude conditions on properties..... 357

I00. Relative frontal area required at altitudes..... $35^{8}$ 
VI. Effects of conditions of use on performance-Continued

C. Methods of shuttering

ror. Detachable sections.

I02. Retractable types.

I03. Side-type radiators.

ro4. Window-blind shutters.

dow-blind shutters..................

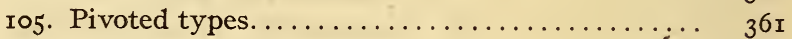

ro6. Regulation of water flow.............. 36 I

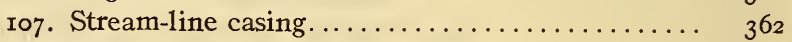

VII. General tables and plots for II typical radiator cores. . . . . . . 362 108. Geometrical characteristics............. 362

ro9. Physical properties of radiators.......... 372

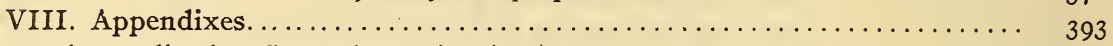

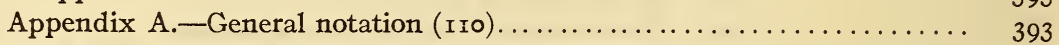

Appendix B.-Turbulence in air tubes................... 393

III. Experimental methods used ........... 393

II2. Pressure gradients................. 394

II3. Cooling coefficients of radiators.......... 394

Ir4. Cooling of wires in an air stream.......... 395

II5. Temperature gradients in air tubes......... 397

Appendix C.-Reduction for air density in computation of air-flow con-

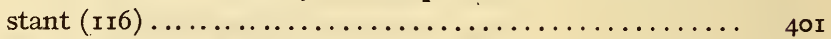

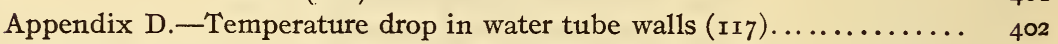

Appendix E.-Choice of temperature difference to be used in expressing results of heat dissipation measurements (II8)...... 402

Appendix F.-Derivation of equation for effectiveness of indirect cooling surface.

II9. Statement of problem. . . . . . . 404

I20. Fin in thermal contact on both sides............ 404

I2 I. Fin in thermal contact on one side only...... 406

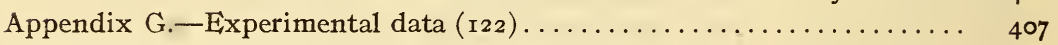

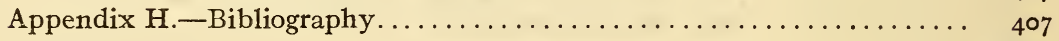

IX. Index . . . . . . . .

\section{INTRODUCTION}

I. INTRODUCTION.-The function of a radiator for an aircraft engine is to maintain the temperature of the water in the cylinder jackets within a specified range, and the first requirement of the radiator is that it shall dissipate heat at a suitable rate. The necessary rate varies for a given engine and radiator as the power developed by the engine changes, while the capacity of the radiator for dissipating heat varies with the speed of flight, position on the aircraft, and the density and temperature of the air. The speed of the craft, and consequently the rate at which air passes through the radiator, is much greater for level flight at full power than for climb at maximum rate. Atmospheric temperatures vary widely between summer and winter and between low and high altitudes, and the density of the air falls off rapidly with altitude, being about one-half as great at 20 ooo feet as at sea level. In general, 
unless a supercharging engine is used, ${ }^{1}$ the most severe conditions for the radiator are found when climbing at maximum rate near the ground in hot weather, when the engine is developing maximum power and both the speed of flight and the temperature difference between the air and the water in the radiator are low. Accordingly, the radiator is usually designed for this condition and provided with some means of shuttering to control its cooling capacity when under less severe conditions. But while capacity for dissipating heat is the first requirement, it is by no means the only one to be considered, for the radiator causes adverse effects on the aircraft which can not be overlooked. It adds somewhat to the weight of the structure, and it adds considerably to the air resistance and consequently to the power required to drive the craft. The conditions most favorable to heat transfer are, in general, such as to cause high air resistance, and the problems of design involve reconciling the antagonistic requirements of high heat transfer and low resistance.

Other factors, which must be considered by the designer but which will not be treated in detail in this paper, include such practical points as strength, ease of repair, and cost of production, and such adverse effects as obstruction of the pilot's view, modifications in the structure of the craft necessary to accommodate the radiator, and, in military machines, liability to injury from hostile fire.

The laws of surface friction for air flow and of heat transfer in long smooth tubes are fairly well known, but the aircraft radiator, which is usually a group of short and sometimes irregular tubes, presents a problem rather different from that of single long tubes, on account of end conditions. The points to be considered in the study of radiators may be grouped about three quantities-flow of air through the core of the radiator, head resistance, and rate of dissipation of heat.

The air flow evidently depends upon speed of flight; density of the air; geometrical form of the radiator; the position in which it is mounted and the effects of other parts of the aircraft, particularly the propeller; nature of the cooling surfaces; and effects of turbulence devices, if used. Head resistance depends upon all the factors mentioned for air flow, and may further be considered in two aspects: As the actual force on the radiator and as head

I If a supercharging engine is used, the most severe conditions for the radiator may be found at high altitudes. 
resistance "chargeable to the radiator," which is the excess of resistance of the whole craft over what it might have been if no radiator had been required. Heat transfer evidently takes place in three stages - from water to metal, through the metal, and from metal to air-the third stage being the one that requires most careful consideration. The rate of heat transfer depends upon the rates and turbulence of water flow and air flow, temperature differences, nature of the cooling surfaces, and the geometrical form of the radiator.

From the above statements it will be seen that the performance of a radiator is determined by external conditions under which it operates and by its geometrical form and physical construction, and can be represented by what may be called its properties, such as air flow through the core, head resistance, and heat dissipation. The general excellence of a radiator will be denoted by a property called "figure of merit," which will be defined as the ratio ${ }^{2}$ of the rate of dissipation of heat to the power absorbed in overcoming the head resistance and sustaining the weight.

The purpose of this report is to show the relations between the conditions under which the radiator operates, its characteristics of form and construction, and the properties that describe its performance, together with a detailed description of the experimental work on which the conclusions are based. The limitations of such a treatise in its immediate application, without any intervening step, to certain problems of design is the obvious one imposed by the impossibility of predicting for each possible case in actual practice the conditions which will determine the air flow. All of the work discussed in this paper and the results of the measurements recorded can be applied, provided only that the air flow through the core be known. The work of this investigation has been extended one step more, namely, to obtain results in terms of flying speed when the radiator is mounted in an unobstructed position. But for obstructed positions it is clear that the problem multiplies indefinitely, because there will be as many cases as there are differences of combination of position and shape of all the parts of an airplane in the path of the air stream through the radiator. Thus, it has proved impossible to do more for the reader who is interested in the performance of a nose radiator (the most common American position) than to set forth the relations to which reference is made at the beginning of this paragraph, leaving

\footnotetext{
${ }^{2}$ Both expressed in the same unit of power.
} 
to him their application when he has secured a suitable measurement or estimate of the airflow characteristic of the particular nose installation with which he is concerned. The physical quantities used in the report have the following significance:

\section{A. DEFINITIONS}

2. CONDITIONS UNDER Which THE RAdiator WORKS.-Flying speed will be taken as the speed of the aircraft relative to the air through which it is flying. Speed of flight relative to the earth would be meaningless in considering the radiator unless wind speeds were known. Unless otherwise stated, flying speed will be expressed in miles per hour (or meters per second).

Density of air will be expressed in pounds per cubic foot (or ${ }^{3}$ $\left.\mathrm{kg} / \mathrm{m}^{3}\right)$.

Temperature differences between the air and the water in the radiator will be taken, for reasons explained below, as the difference in degrees between the average of the temperatures of the water on entering and leaving the radiator and the temperature of the air at entrance.

Altitude above sea level will be expressed in feet (or meters), and estimated atmospheric conditions at altitudes will be taken from Figs. 46-47, which are based on data given by W. R. Gregg in the Monthly Weather Review, January, I9I8, for four stations in the United States.

The positions in which a radiator may be mounted on the aircraft will be divided into three classes-unobstructed, obstructed, and slip-stream positions.

Unobstructed positions are those in which the flow of air through and around the radiator is practically unaffected by other parts of the structure.

Obstructed positions are those in which the flow of air through the radiator is reduced by the effects of other parts of the structure.

Slip-stream positions are those in which the blast from the propeller blows over the radiator. They include, therefore, both positions which, but for the propeller, would be unobstructed and those which even in the absence of the propeller would be obstructed.

3. Characteristics That Identify the Radiator Core.The core of the radiator is that part through which air passes, as distinguished from the water tanks or headers. In order to elim-

${ }^{8}$ The same as $\mathrm{g} / \mathrm{1000} \mathrm{cm}^{8}$. 
inate size of radiator from the comparisons between types, all characteristics will be reduced to unit frontal area of core. ${ }^{4}$

Depth of core is the linear dimension in the direction of air flow and will be expressed in feet or in inches (or $\mathrm{cm}$ ).

Length of core is the linear dimension in the general direction of water flow and will be expressed in feet (or $\mathrm{cm}$ ).

Width of core is measured perpendicular to both length and depth and will be expressed in feet (or $\mathrm{cm}$ ).

Direct cooling surface includes all surfaces backed by flowing water and from which heat is dissipated directly to the air. Cooling surface, whether direct or indirect, is usually expressed in square feet per square foot (or $\mathrm{m}^{2}$ per $\mathrm{m}^{2}$ ) of frontal area of core. In this form it is a ratio independent of the unit of area used, and in this paper it will be so expressed as a ratio.

Indirect cooling surface will be defined as any cooling surface not backed by flowing water. It may be formed by "fins" projecting into the air passages or by the walls of the water tubes at points where pockets are formed, so that the water is more or less stagnant. The important difference between direct and indirect cooling surface lies in the fact that as heat passes from the water to the air it is conducted in the case of direct cooling surface only through the thickness of the metal, while in the case of indirect cooling surface it must be conducted along the metal for a distance much greater than its thickness. Since this conduction is possible only when the temperature of the metal falls off with increasing distance from the source of heat, indirect cooling surface must have a lower mean temperature than direct surface adjacent to it.

Effectiveness of indirect cooling surface will be defined as the ratio of its rate of dissipation of heat to that of an equal area of direct surface under the same conditions of air flow, the direct surface being at a temperature equal to that of the parts of the indirect surface that are adjacent to its source of heat.

Free area is the ratio of the average total area of the cross section of the air passages to the frontal area of the core.

Weight of the core, either empty or including the contained water, will be expressed in pounds per square foot (or $\mathrm{kg} / \mathrm{m}^{2}$ ) of frontal area.

Water content is the weight of water contained in the core and will be expressed in pounds per square foot (or $\mathrm{kg} / \mathrm{m}^{2}$ ) of frontal area.

${ }^{4}$ Quantities so expressed may evidently be reduced to unit cooling surface if desired by dividing by the number of units of cooling surface in a section of core of unit frontal area. 
Hydraulic radius of a water tube or of an air tube is the quotient of its cross sectional area by the perimeter of such a section and will be expressed in feet or inches (or $\mathrm{cm}$ ).

4. Properties That Define the Performance of a RadiATOR.-The properties that indicate the performance of a radiator will be reduced to unit frontal area of core, and will be defined as follows:

Air flow or mass flow of air is the quantity rate of passage of air through the core and will be expressed in pounds per second per square foot $\left(\right.$ or $\mathrm{kg} / \mathrm{sec} / \mathrm{m}^{2}$ ) of frontal area.

Air flow constant of a radiator or core is, for unobstructed positions, the fractional part of the air directly approaching the radiator or core that passes through it or unity minus the fraction deflected around it. For example, if the air has such a speed relative to the radiator as would deliver ro pounds of air per second for each square foot of cross sectional area of the stream, but only 7 pounds per second pass through each square foot of core, while 3 pounds out of every Io are deflected around it, then the air flow constant is 0.7 . For obstructed and slip-stream positions the air flow constant is equal to the number of pounds of air flowing per second through a square foot of frontal area of core, divided by the product of the flying speed in feet per second and the density of the air in pounds per cubic foot. ${ }^{3}$ The air flow constant is usually less than unity, although in slip-stream positions it may exceed that value.

The air flow constant is the factor $m$ of the equation

$$
M=m \rho S^{\prime},
$$

where $M=$ air flow, $1 \mathrm{~b} . / \mathrm{sec} . / \mathrm{ft}^{2}{ }^{2}$ (or $\mathrm{kg} / \mathrm{sec} . / \mathrm{m}^{2}$ ).

$$
\begin{aligned}
& m=\text { air flow constant, } \\
& \rho=\text { air density, } 1 \mathrm{~b} . / \mathrm{ft} .^{3} \text { (or } \mathrm{kg} / \mathrm{m}^{3} \text { ), } \\
& S^{\prime}=\text { flying speed, } \mathrm{ft} . / \mathrm{sec} \text { ( or } \mathrm{m} / \mathrm{sec} \text {.). }
\end{aligned}
$$

Head resistance of the core is the resistance that it offers to passage through the air and is equal to the force required to push it through the air at constant speed. Head resistance will be expressed in pounds per square foot $\left(\mathrm{or} \mathrm{kg} / \mathrm{m}^{2}\right.$ ) of frontal area.

Head resistance constant will be defined as the factor $c^{\prime}$ of the approximate equation

$$
R^{\prime}=c^{\prime} \rho S^{\prime 2}
$$

where $R^{\prime}=$ head resistance in poundals per square foot (or dynes per $\left.\mathrm{cm}^{2}\right)$,

\footnotetext{
sAny other consistent set of units would servc equally well.
} 
$\rho=$ air density in pounds per cubic foot $\left(\right.$ or $\left.\mathrm{g} / \mathrm{cm}^{3}\right)$,

$S^{\prime}=$ speed relative to the $\operatorname{air}^{6}$ in feet per second (or $\mathrm{cm} / \mathrm{sec}$.).

Throughout this report head resistance has been reduced to an air density of 0.0750 pounds per cubic foot $\left(\mathrm{r}^{\prime} .204 \mathrm{~kg} / \mathrm{m}^{3}\right)$, and in most cases it will be convenient to use a constant called the "head resistance factor," which will be defined as the factor $c$ of the approximate equation

where

$$
R=c S^{2} \text {, }
$$

$R=$ head resistance in pounds per square foot (or $\mathrm{kg} / \mathrm{m}^{2}$ ) frontal area at an air density of 0.0750 pound per cubic foot $\left(\mathrm{r} .204 \mathrm{~kg} / \mathrm{m}^{3}\right)$,

$c=$ head resistance factor,

$S=$ speed relative to the air, in miles per hour (or $\mathrm{m} / \mathrm{sec}$.). It is evident that the numerical value of the "head resistance factor" will depend upon the units used.

Head resistance chargeable to the radiator is the difference between the resistance of the entire aircraft with its radiator (under given external conditions, such as speed and air density) and the resistance that it might have had if it could have been designed without a radiator. This includes the resistance of the core, the resistance of headers and exposed connecting pipes, and any increase in total resistance caused by mutual effects of the radiator and its surroundings, or by changes in the design of the structure necessitated by the presence of the radiator. For example, with engines of high power cooled by a radiator in the nose of the fuselage, the radiator may need to be so large in order to cool the engine as to require enlargement of the fuselage to contain it. When expressed numerically, head resistance chargeable to the radiator will be expressed in the same units as head resistance of the core, namely, pounds per square foot (or $\mathrm{kg} / \mathrm{m}^{2}$ ) of frontal area of core.

Power absorbed by the core is the power used in overcoming its head resistance and sustaining its weight. It will be expressed in horsepower per square foot (or $\mathrm{kw} / \mathrm{m}^{2}$ ) of frontal area.

Power absorbed chargeable to the radiator corresponds to head resistance chargeable to the radiator and is the difference between the power required to drive the aircraft under given external conditions, such as speed and air density, and the power that would have been needed if it could have been designed and operated

\footnotetext{
"Speed of the radiator relative to the air, which is the same as "flying speed" if the radiator is in an unobstructed position.
} 
without a radiator. When it is expressed numerically, the unit used will be horsepower per square foot (or $\mathrm{kw} / \mathrm{m}^{2}$ ) of frontal area of core.

Heat dissipation will be used as the term for rate of dissipation of heat by the radiator. Unless otherwise stated, it will be expressed in horsepower per square foot (or $\mathrm{kw} / \mathrm{m}^{2}$ ) of frontal area of core, per $100^{\circ} \mathrm{F}$ (or $50^{\circ} \mathrm{C}$ ) difference between the average of the temperatures of the water on entering and leaving the radiator and the temperature of the entering air.

Figure of merit is the ratio, when both are expressed in the same unit of power, of the heat dissipation to the power absorbed. The figure of merit of the core will then be the ratio of the heat dissipation to the power absorbed by the core, while the figure of merit of the radiator as a whole (which may or may not be equal to that of the core) will be the ratio of the heat dissipation to the power absorbed chargeable to the radiator: ${ }^{7}$

\section{AIR FLOW THROUGH THE RADIATOR}

5. General Statement.-The heat dissipated by a radiator is taken up by streams of air flowing through its tubes, and both the quantity of air delivered by the streams and their condition of turbulence are important factors in the dissipation of heat. Since air transmits heat only slowly by conduction, but principally by convection, the amount of heat taken from the metal surface depends very greatly on the number of different molecules of air that come in contact with the metal, and the most rapid transfer of heat requires a considerable scouring of the surface, while a layer of stagnant air acts as an effective insulator. But the collision of molecules of air with those of the metal, imparting heat to the air because of the molecular motion in the hot metal, also tends, because of the mass motion, to drag the air along with the radiator instead of allowing it to pass through the tubes; and while turbulence in the air streams facilitates heat transfer, it also increases surface friction, and thereby head resistance.

It is well known that at points not too near the ends in long tubes with smooth walls air flow is of two kinds, depending upon the relations between diameter of the tube and the speed, viscosity, and density of the air. At low speeds the flow is practically along

7 This figure of merit must not be confused with the "figure of merit" used by the British, which refers to an arbitrarily chosen radiator as a standard, nor with that used by the French, which is the metric horsepower absorbed while dissipating heat at the rate of 1000 kilogram calories per minute. If the number $8 \mathrm{r} .0$ is divided by the French figure of merit, the quotient will be the figure of merit as used in this country for a temperature difference between air and water (as defined above) of $100^{\circ} \mathrm{F}$. 
stream lines parallel to the walls of the tubes and is called "viscous" or "stream-line" flow, while at higher speeds in the same tube the flow is broken up into vortices and is called "turbulent" flow. In viscous flow the surface friction or resistance to flow is due principally to the viscosity of the air and is roughly proportional to the first power of the speed, while in turbulent flow viscosity is of less importance than density, and the resistance is roughly proportional to the square of the speed. The shortness of the tubes of radiators and the irregular and broken forms often employed make it unsafe to apply the theory of long tubes except with considerable caution; but the qualitative ideas of viscous and turbulent flow are very helpful in visualizing the flow in the radiators, and it may be expected that in a cluster of tubes such as a radiator conditions corresponding to long tubes may be found much nearer the ends than in a single tube. Unless some kind of a mouthpiece is provided air coming over the edges of a single tube enters from many directions, and the same is true to some extent of radiator tubes that are near the edges of the section; but the air that enters tubes near the center of the section is confined by that entering the other tubes and is fairly well directed even before it reaches the tubes.

An investigation of the character of flow in the air tubes of the radiators indicated that turbulence exists, even in tubes with straight, smooth walls, and that in different types of radiator the turbulence varies considerably in nature or in degree. The experimental evidence was obtained from pressure gradients, surface cooling coefficients of radiators, cooling of wires in air streams, and temperature gradients across the air passages. Details of the method used and the results obtained are given in Appendix B.

The amount of air passing through the radiator tubes, which throughout this report will be understood to mean a mass flow rather than linear speed, is governed by the difference in pressure between the front and rear faces of the radiator, the speed of flight, the density of the air, and the geometrical characteristics of the tubes, and especially the ends of the tubes, which may have a considerable effect. In addition to these factors other parts of the structure in the vicinity of the radiator may greatly reduce the air flow by offering obstructions to the stream either in front of or behind the radiator. The effect of obstructions behind the radiator is often underestimated by the novice. 


\section{A. EXPERIMENTAL METROD OF MEASURING AIR FLOW AND COMPU- TATION}

6. WIND TUNNELS.-Measurements of air speed were made by means of pitot tubes, each tube being compared with at least one other that was known to give readings correct within the limit of experimental errors. The wind tunnels in which the tests were made will be described in detail below, in the chapters on head resistance and heat transfer, while in this section only enough description will be given to explain the measurement of air flow. Three tunnels were used-one of square cross section, 8 inches $(20.3 \mathrm{~cm})$ on a side, the entire tunnel inclosed in a steel tank, another of square cross section, 8 inches on a side, but not inclosed, and a third of octagonal section, 54 inches $(\mathrm{I} 37 \mathrm{~cm})$ between parallel sides. The steel tank could be closed and the air partially exhausted from it for tests at reduced pressure, and the (8-inch) tunnel inclosed in it will be called, for convenience in brief reference, the "reduced-pressure tunnel." In the" uninclosed 8-inch tunnel superheated steam was provided as a source of heat for the calorimetric tests, and this tunnel will be called the "steam tunnel." Reference to the 54 -inch tunnel will be made either by that name or as the "open tunnel," since the radiator section does not fill it, as it does the others, and the condition is similar to mounting in the open air.

\section{(a) STEAM TUNNEL}

7. APPARATUS.-Fig. I shows the form of section of radiator used for tests.in the reduced pressure and steam tunnels. The core is 8 inches $(20.3 \mathrm{~cm})$ square, so that it just fills the air channel, while the water tanks or headers are outside of the air stream, and the mass flow of air through the core can be determined by measuring the flow past any section of the tunnel. ${ }^{8}$ This measurement was made by means of a "pitot grid" and a piezometer ring, connected to a differential pressure gage, and indicated in Fig. 3. The pitot grid consisted of 16 dynamic pressure openings arranged at equal intervals in a plane, as shown in Fig. 4, and all connected to a single tube leading to the gage. The piezometer ring was constructed as follows: Sheet brass was inlaid in the walls of the tunnel for a distance of $25 \mathrm{~cm}$ (Io inches) before and behind the radiator in order to furnish a smooth surface. Square copper tubes were soldered to the outside of the brass sheets,

${ }^{8}$ If the linear speed of the air in the core of the radiator is desired, it can evidently be computed from the mass flow, the density, and the "free area" of the core. 
around the perimeter of the section at which the dynamic openings of the grid were placed, which was $15 \mathrm{~cm}$ ( 6 inches) in front of the front face of the radiator, and holes about $0.5 \mathrm{~mm}(0.02$ inch) in diameter were drilled from the outside through the tube

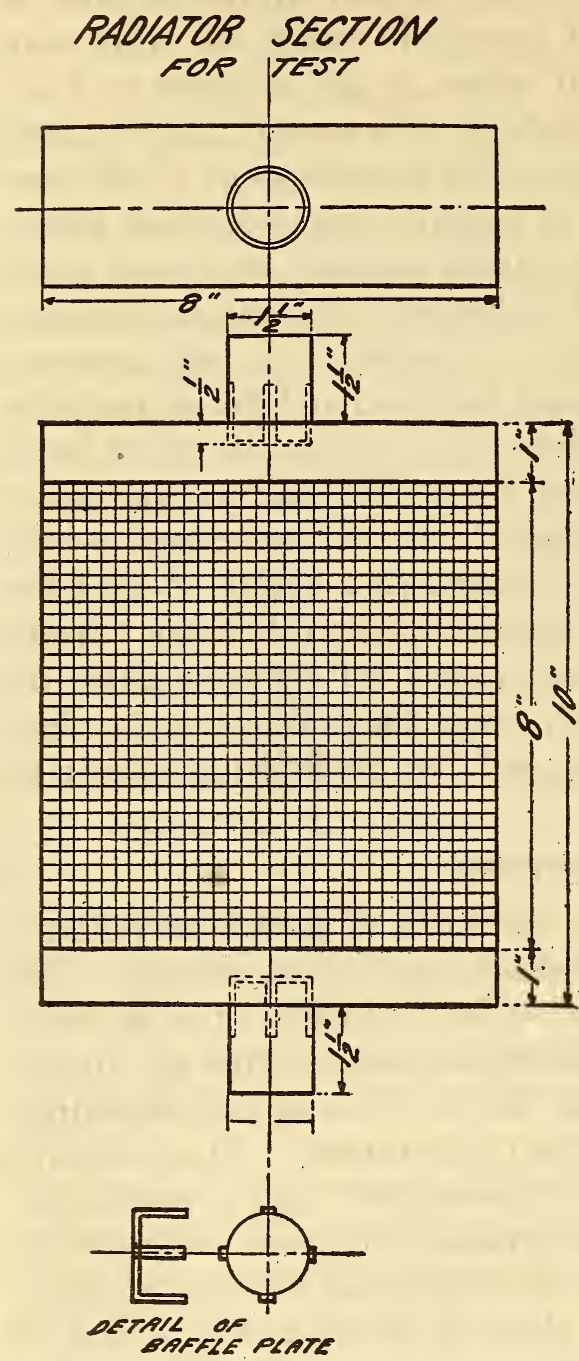

FIG. I.-Radiator section prepared for calorimetric test. and the sheet at intervals of I. $3 \mathrm{~cm}$ (0.5 inch) around the channel. The burr was scraped off from the inner surface, the holes cleaned and smoothed with a hand drill, and then the holes in the outer side of the copper tube were closed with solder. Similar piezometer rings were placed at sections $2.5 \mathrm{~cm}$ (I inch) in front of and 2.5 and $15 \mathrm{~cm}$ (I and 6 inches) behind the radiator for the measurement of differences in static pressure in the air stream passing through the radiator.

This arrangement is the one used for measuring air flow during calorimetric tests. For the work with a secondary velocity gage, denominated the "pressure tube" (art. I5), an additional section of tunnel was inserted, bringing the dynamic pressure openings of the front pitot grid $36 \mathrm{~cm}$ (I4 inches) from the face of the radiator.

8. Calibratrons.-The pitot grid and its piezometer ring were calibrated, and the distribution of speed across the channel was determined by comparison with a movable pitot-static tube placed at different positions over the section. Simultaneous readings were taken of the movable pitot-static tube and the grid. The mean speed in the channel was computed with the aid of Simpson's rule from the readings of the movable tube and was compared 
Technologic Papers of the Bureau of Standards, Vol. 16

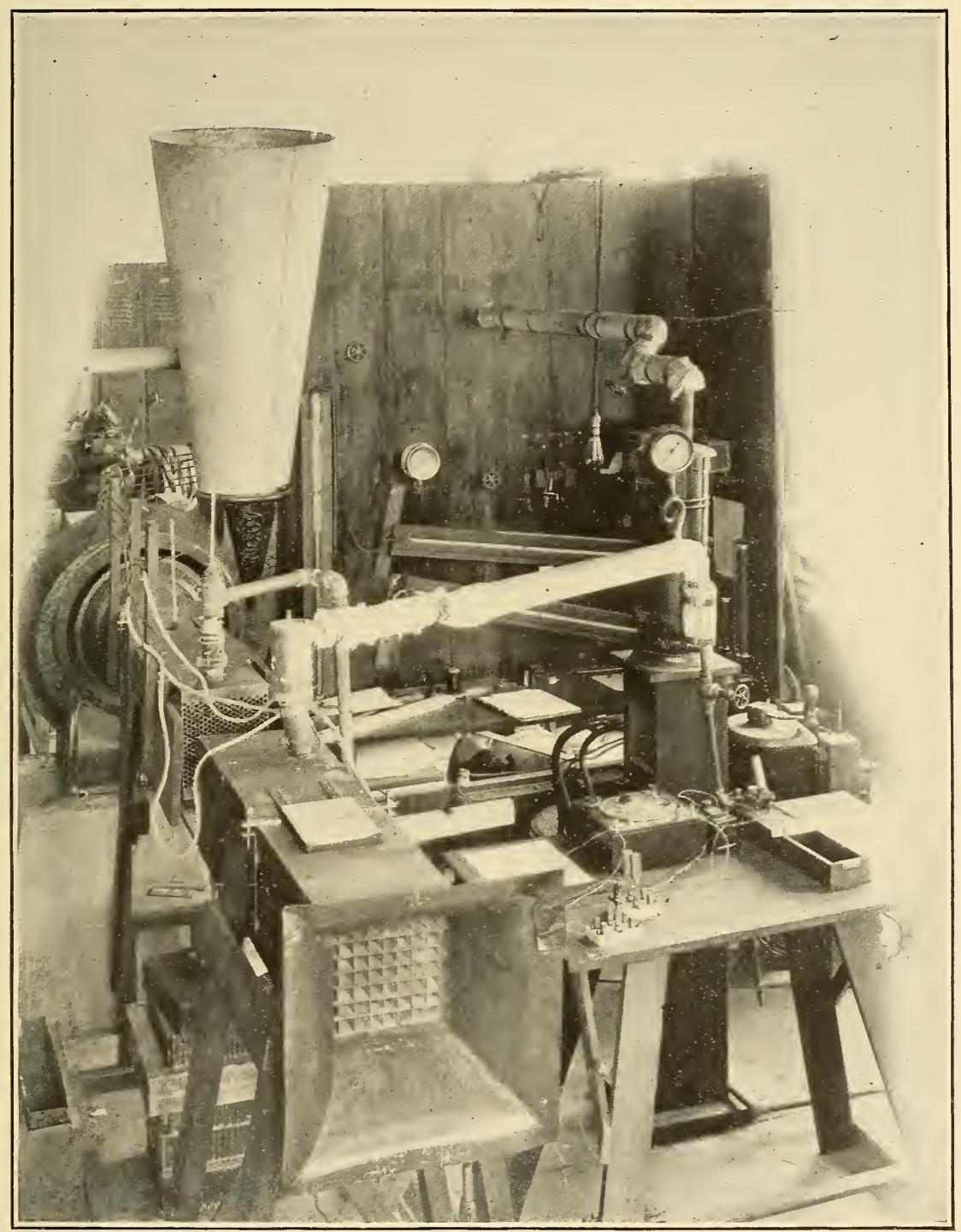

Fig. 2.- Small wind tunnel $20 \mathrm{~cm}(8 \mathrm{in}$.) square and accessory apparatus for calorimetric tests of radiator cores

Test core with hexagonal cells is shown at the opening near the center of the tunnel. Hot water or steam connections furnish the heat supply 

with the readings of the grid and piezometer ring. 'The steam tunnel was used in two forms. When first built, air was pushed through it by a blower, but after a time it became necessary to move the apparatus to another building, and it was then rebuilt as a suction tunnel. The explorations in the first tunnel showed that at seven different speeds covering the range of experimental use the indications of the grid were within about I.5 per cent of the mean air speed, the errors being greatest at the lower speeds, at which the readings were least reliable. In the rebuilt tunnel the exploration was less satisfactory, for the movable pitot tube was only about $12 \mathrm{~cm}$ (5 inches) behind the grid, and its readings were

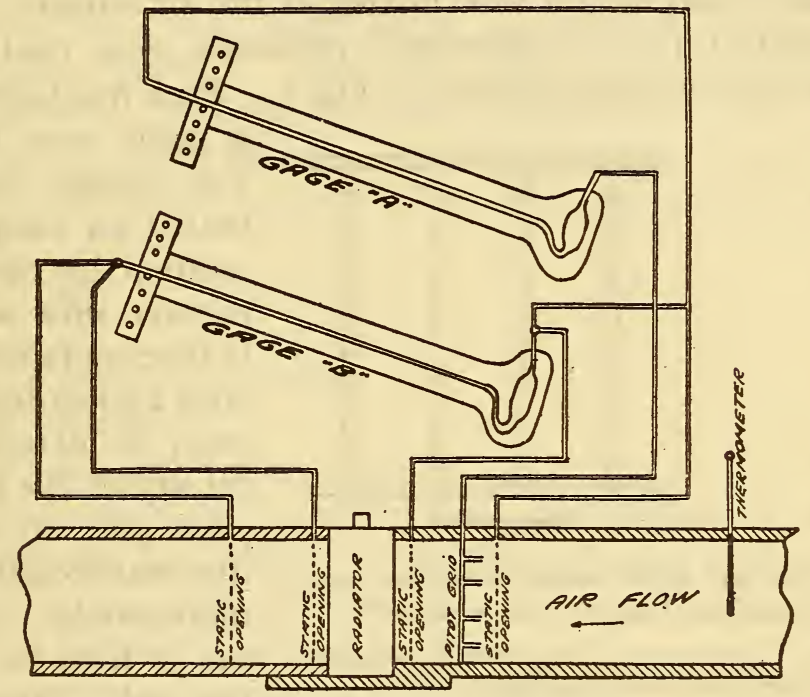

FIG. 3.-Schematic diagram of calorimetric wind tunnel (" steam tunnel"'), $20 \mathrm{~cm}$ ( 8 in.) square

Used for heat transfer measurements at wind speeds up to 30 meters per second ( 68 miles per hour)

affected by the crossbars of the latter, but since the same grid was used and the conditions for uniform velocity distribution appeared better than in the first tunnel it seemed unnecessary to make an exploration at some other section, which would have involved cutting an opening in the wall. The correctness of the readings of the pitot grid and piezometer ring was established by the agreement between values of heat dissipation computed from the rate of flow and rise in temperature of the air and from the rate of flow and fall in temperature of the water during regular calorimetric tests.

A further indication of the straightness of flow in the channel was obtained by the use of ammonium chloride smoke introduced $75013^{\circ}-22-2$ 
into the tunnel through a glass tube about $8 \mathrm{~mm}\left(\frac{5}{16}\right.$ inch) in diameter, which projected through the bell mouth of the tunnel to a few centimeters beyond the straightening honeycomb at its entrance. On looking into the tunnel, either down stream through the mouth or across the stream through a window in the top, the smoke was seen to follow a straight course down the stream, with very little spreading for some distance. When a radiator was placed in the tunnel, the smoke was found deposited over a certain area of the face, which was fairly well defined, rather than shading off very gradually. At a distance of I meter from the mouth of the smoke tube the areas ranged from 4 to 9 per cent of the cross section of the tunnel, indicating a slow mixing of the air stream.

9. OBSERVATIONS.-Differential pressures were read on two inclined water gauges indicated in Fig. 3. Each was hinged about
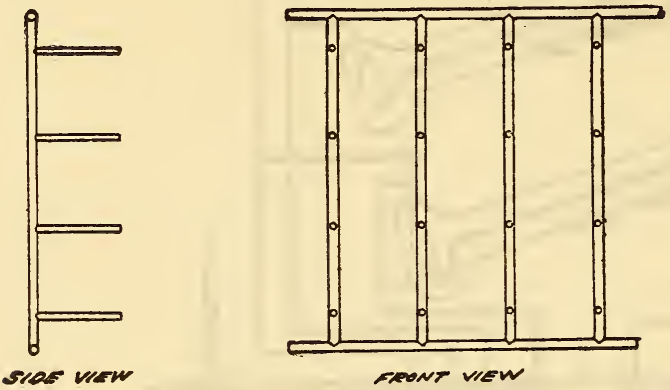

FIG. 4.-Pitot grid for the measurement of air velocity in the small wind tunnel ("steam tunnel")

Transmits to a water gage the average dynamic pressure at 16 points in the cross section $20 \mathrm{~cm}(8 \mathrm{in}$.) square a point near the bulb. The gauges were calibrated for each angular position by direct comparison with a vertical $U$-tube containing a light oil of known density. In order to determine the density of the air at the pitot grid, its temperature was measured on a thermometer about 60 $\mathrm{cm}$ (2 feet) in front of the grid. Pressure was

determined by the reading of a barometer in the room, corrected by the amount of the rise or fall of pressure between the room and the piezometer ring (measured on one of the inclined gauges by connecting one side to the ring and leaving the other side open), and approximate relative humidity was read on a recording hygrometer. The errors in humidity were sometimes as high as Io or I 5 per cent, but the effect of humidity enters the computations only as a very minor correction, and even errors of I $_{5}$ per cent in humidity measurement may safely be neglected.

ro. Computation.-For the computation of air flow the equation of the pitot tube

was put in the form

$$
v=\sqrt{2 g h}
$$

$$
M=c \sqrt{x \rho},
$$


where $\quad v=$ linear air speed,

$g=$ acceleration of gravity,

$h=$ "head" of air (units of length),

$\rho=$ density of air,

$M=$ air flow, ${ }^{9}$

$x=$ reading on the gage,

$c=\mathrm{a}$ conversion factor, depending upon the units used and the calibration of the gage.

The density of the air was computed by either of two methods. In the first method the pressure and temperature were converted to pounds per square inch and degrees Fahrenheit, and density was taken from a chart given by W. C. Rowse, ${ }^{10}$ which includes correction for humidity. This chart could not be used, however, for low pressures, and the following method for use with the reduced pressure tunnel was also used with the steam tunnel.

The density of dry air may evidently be obtained from the equation

$$
\rho_{\mathrm{d}}=\frac{b p}{T}
$$

where $\rho_{\mathrm{d}}=$ density of dry air,

$p=$ pressure,

$T=$ absolute temperature,

$b=a$ constant, depending upon the units used.

Also considering any constant temperature $T$, let

$p=$ atmospheric pressure,

$p_{\mathrm{d}}=$ partial pressure of air (dry)

$p_{\mathrm{v}}=$ partial pressure of water vapor,

$p_{\mathrm{s}}=$ pressure of saturated water vapor at temperature $T$,

$\rho=$ density of mixture,

$\rho_{\mathrm{d}}=$ density of dry air at pressure $p$, and temperature $T$,

$\rho_{\mathrm{a}}=$ density of dry air at pressure $p_{\mathrm{d}}$ and temperature $T$,

$\rho_{\mathrm{v}}=$ density of vapor at $p_{\mathrm{v}}$ and $T$,

$\rho_{\mathrm{s}}=$ density of saturated vapor at temperature $T$,

$r=$ relative humidity,

$c=$ correction for humidity such that,

$\rho=\rho_{\mathrm{d}}+c$.

From Dalton's law

$$
p=p_{\mathrm{d}}+p_{\mathrm{v}}
$$

${ }^{9}$ It is important to bear in mind that, unless otherwise stated, air flow has in every case been reduced to unit time and unit frontal area of core.

10 Transactions, Amer. Soc. of Mech. Engs., I913, p. 690. 
and from the definitions of density and relative humidity

$$
\rho=\rho_{\mathrm{a}}+r \rho_{\mathrm{s}},
$$

while by Boyle's law

$$
\rho_{\mathrm{a}}=\rho_{\mathrm{d}} \frac{p_{\mathrm{d}}}{p}
$$

also

$$
p_{\mathrm{v}}=r p_{\mathrm{s}} \text {. }
$$

Substituting equations (4) in (3) and (5) in (2),

$$
\begin{gathered}
\rho=\rho_{\mathrm{d}} \frac{p_{\mathrm{d}}}{p}+r \rho_{\mathrm{s}}, \\
p=p_{\mathrm{d}}+r p_{\mathrm{s}} .
\end{gathered}
$$

Equations (I), (6), and ( 7 ) contain the three unknown quantities $\rho, p_{\mathrm{d}}$, and $c$. Solving for $c$,

$$
c=r\left(\rho_{\mathrm{s}}-\rho_{\mathrm{d}} \frac{p_{\mathrm{s}}}{p}\right)
$$

The quantity in parentheses in equation (8) is a function of the temperature only, and for convenience in computation a chart (Fig. 5) was made showing this quantity as a function of the temperature.

(b) REDUCED PRESSURE TUNNEL

II. Apparatus.-Figure 6 shows a diagram of the reduced pressure tunnel, which, in the sections adjoining the radiator, was similar to the steam tunnel. At first a Thomas meter was used to measure the air flow, but the velocity distribution was poor, and at high air speeds the meter gave large errors and was finally discarded. In its place a pitot grid and piezometer rings were used, similar to those described above for the steam tunnel. With the addition of honeycombs to straighten the flow, the pitot grid and its piezometer ring were found by an exploration similar to that made in the steam tunnel to indicate speed correctly within I. 5 per cent at all speeds used.

For the measurement of pressure a barometer was connected directly to the piezometer ring corresponding to the pitot grid. Temperature of the air in the channel was measured by an electric resistance thermometer of No. 40 nickel wire (diameter $0.08 \mathrm{~mm}$, 3 mils) inclosed in $0.5 \mathrm{~mm}$ ( 0.02 inch) copper tube and constructed in the form shown in Fig. 7. The thermometer and the Wheatstone bridge used to read it were calibrated by the appropriate sections of the Bureau, and the measurement of temperature could be made to $0.2^{\circ} \mathrm{C}$. 


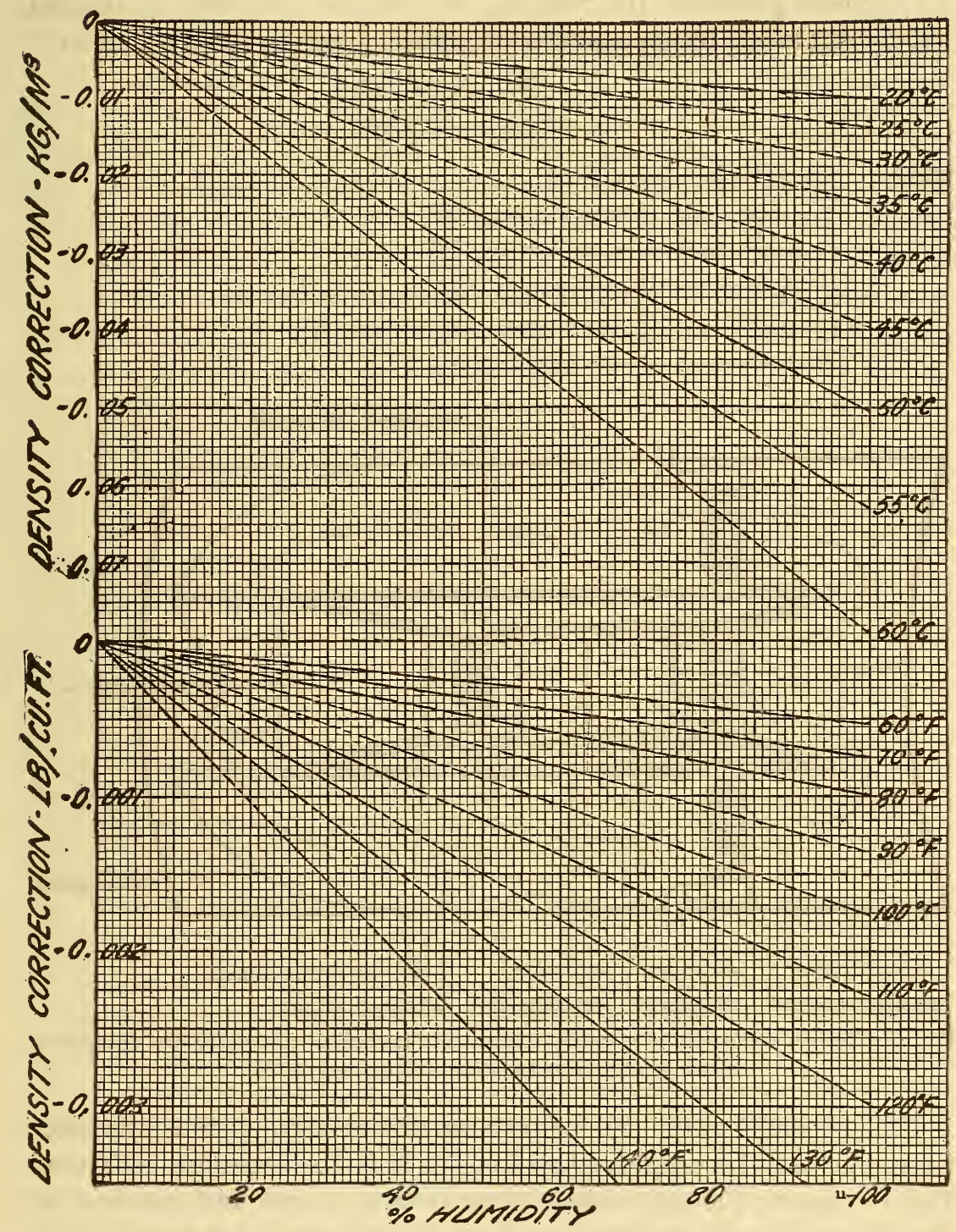

FIG. 5.-Correction term to be added (negatively) to the density of dry air at $760 \mathrm{~mm}$ barometric pressure and given temperature in order to obtain the density of humid air at various humidities 
I2. HumIDITY.--Relative humidity was determined from the readings of a wet-and-dry bulb hygrometer inside of the tank containing the tunnel by means of an ordinary chart for the purpose. At reduced pressures the readings of the chart required correction which was made by means of an equation given by W. H. Carrier. ${ }^{11}$

$$
r=\frac{e^{\prime}}{e_{2}}-\frac{\left(P-e^{\prime}\right)\left(t-t^{\prime}\right)}{(2800-\mathrm{T} \cdot 3 t) e_{2}}
$$

where $r=$ relative humidity,

$t=$ dry bulb temperature, ${ }^{\circ} \mathrm{F}$,

$t^{\prime}=$ wet bulb temperature, ${ }^{\circ} \mathrm{F}$,

$e^{\prime}=$ pressure of saturated vapor at $t^{\prime}{ }^{\circ} \mathrm{F}$,

$e_{2}=$ pressure of saturated vapor at $t{ }^{\circ} \mathrm{F}$,

$P=$ pressure of air and vapor.

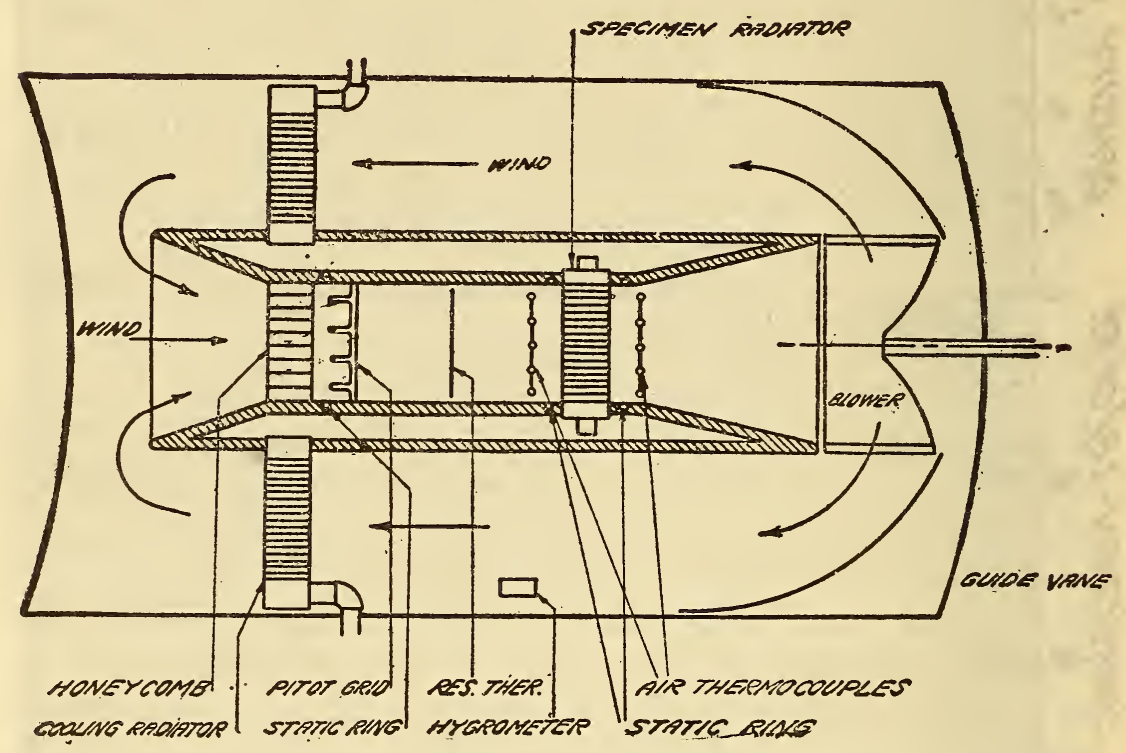

FIG. 6.-Schematic diagram of reduced pressure wind tunnel for measuring heat dissipation under conditions simulating high altitude flight

The pressures enter the formula as ratios only, so that any unit whatever may be employed so long as it it the same for all three. The remaining factors, temperature terms, are not independent of the choice of units, the 2800 and I.3 being correct for the Fahrenheit scale. The formula was converted into units convenient for the present use, and the factor

$$
\frac{i-t^{\prime}}{(2800-I .3 t) e_{2}}
$$

II Transactions, Amer. Soc. of Mech. Engs., I9II, p. rozo. 
was plotted as a function of $t$ and $\left(t-t^{\prime}\right)$ for convenience in computation. Having determined pressure, temperature, and humidity, the density of the air was computed as described for the steam tunnel.

(c) OPEN TUNNEL

I3. ApPaRATUS.-The measurement of air flow in the 54-inch $(\mathrm{I} 37 \mathrm{~cm}$ ) open wind tunnel presented quite a different problem from that of the 8-inch closed tunnels. In this tunnel the problem was to determine the flow through the core of the radiator in terms of the linear speed of the air stream in which it was placed.

The wind tunnel is illustrated in Figs. 8 and 9. Explorations across the working section showed that when the channel was unobstructed the velocity was uniform to about I per cent at all points not nearer than $20 \mathrm{~cm}$ ( 8 inches) to the wall. The speed of the air stream was measured by a pitot-static tube mounted in the center of the channel at a point about $180 \mathrm{~cm}$ ( 6 feet) in front of the radiator. The mounting of the pitot tube was a hollow brass strut of cross section about $22 \mathrm{~mm}$ $(7 / 8$ inch) in length and $9 \mathrm{~mm}$ $(3 / 8$ inch $)$ in maximum width, rising from the floor of the tunnel and containing the tubes that led to the differential pressure gage. The radiator was mounted on a support rising through the floor of the tunnel, described in detail in the chapter (III) on head resistance (art. 27).

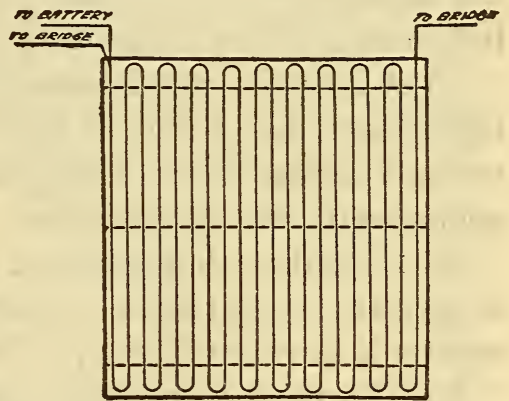

Fig. 7.-Resistance thermometer (electric) for measurement of air temperature in wind tunnel

Nickel wire, $0.08 \mathrm{~mm}$ in diameter, is incased in a copper tube, wound as shown to integrate temperature over the whole cross sectional area of the wind tunnel

To test whether the conditions truly represent a speed in the open air, silk threads were attached to a fine vertical wire strung about $15 \mathrm{~cm}$ ( 6 inches) in front of a large-size section of high head resistance, $\mathrm{G}-3,40.6 \mathrm{~cm}$ ( $\mathrm{I} 6$ inches) square. The threads showed a considerable curvature of the stream lines around and close to the section, but no appreciable curvature within $20 \mathrm{~cm}$ ( 8 inches) of the tunnel wall. It was assumed, therefore, that the effect of the radiator on the air stream was confined well within the section of the tunnel, and that open-air conditions were well represented.

I 4. METHOD OF MEASUREMENT.-The measurement of air flow through the core presented some difficulties, and a number of methods were tried before one was found that gave results at all reliable. 
(a) An attempt was made to measure the flow with a small pitot-static tube behind the core and in positions ranging from well within the cell to a few inches behind the core, but it soon became evident that this method was not practicable because of the turbulence of the air encountered.

(b) Threads were attached to the rear face of the radiator to define the stream, in the hope that the air passing through the core could be followed to a position where the flow would not be too turbulent for the use of a pitot-static tube, and that in such a position it would be possible to measure the speed and the area of cross-section of the air that had passed through the core. It was found, however, that even aside from the difficulty introduced by the tendency of the pull on the threads to straighten them, so that they would not follow the lines of the stream, the flapping of the threads was too great, and consequently their position too indefinite to allow of measurement of the area included by them.

(c) A hot-wire anemometer was tried and gave some promise of fair results, but before it had been thoroughly tested, a simpler method seemed to be giving very fair results, and the use of the anemometer was discontinued.

(d) A method that was used in a large number of cases employed a specially constructed venturi meter, of which a longitudinal section is shown in Fig. II. Its use is described below.

(e) The most satisfactory method used was based on a small tube, which will be called the "pressure tube," stretched through the air passages of the radiators, with a small static pressure opening near its center. This has been used on all cores that present straight air passiges, while the air venturi is still the most satisfactory instrument hat has been used on cores through which the pressure tube can no: be passed.

15. PRESSURE TUBE. - The pressure tube was used in both the steam tunnel (8-inch, $20.3 \mathrm{~cm}$ ) and the open tunnel (54-inch, I37 cm), and its purpose was to furnish a basis of comparison between observations in the two tunnels. Its use is based on the assumption that if air flows through the radiator at a definite rate certain definite pressure differences will be set up in the air passages, whether the radiator is in the steam tunnel, where the flow of air through it is measured, or in the open tunnel, where the speed measured corresponds to speed of flight. In other words, the assumption is made that pressure drop between any two points in an air tube, due to the air current flowing in that tube, is a definite function of the current strength, although not necessarily 


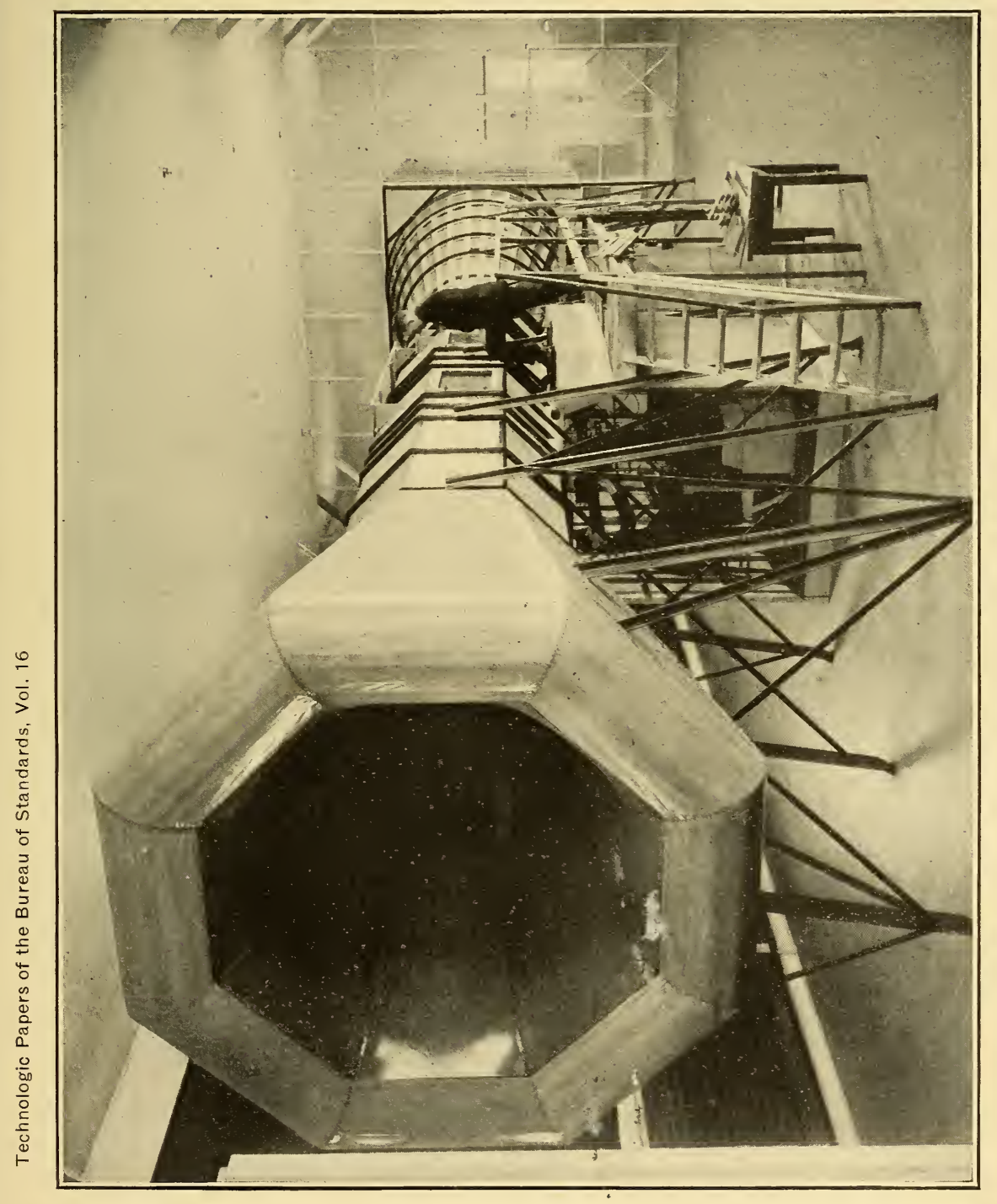

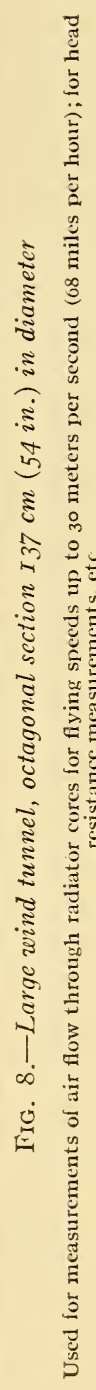



a known function and is single valued, so that whenever a given pressure drop is obtained it is because the same current is flowing in the tube. Operating on this principle, the pressure tube will identify in the core of the radiator when mounted in the open tunnel an air flow of which the numerical value is ascertainable from absolute measurements in the closed (steam) tunnel. This air flow in the core can then be compared with the air flow in the open tunnel at a distance from the radiator and the relation obtained between flying speed and air flow in the core.

The pressure tube was of steel, about I $\mathrm{mm}$ (0.04 inch) outside diameter, 6I cm (24 inches) long, and, as stated above, contained an opening near the center, the plane of this opening being parallel to the air flow, so as to measure static pressure without any com-

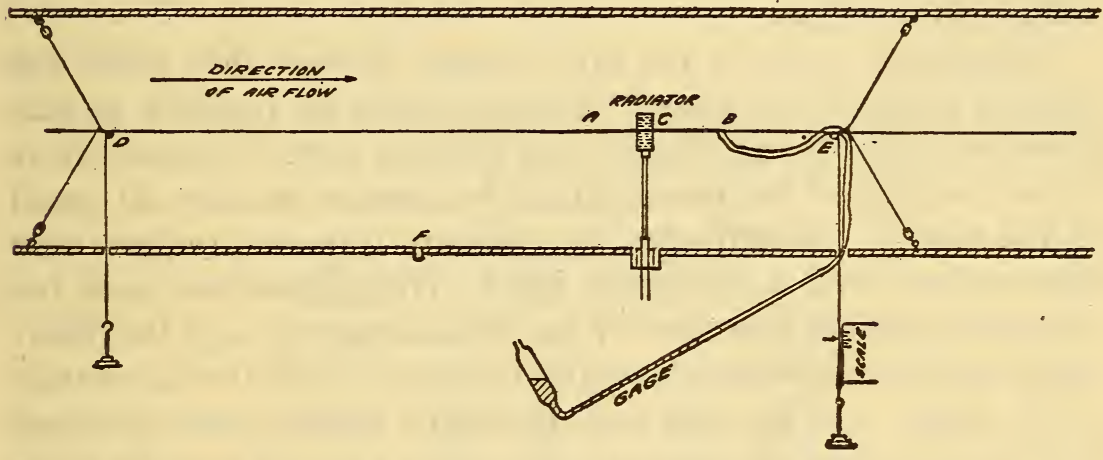

FIG. 9.-Schematic digram of the method of mounting the "pressure tube". through a radiator cell in the large open wind tunnel

ponent of velocity head. The forward (upstream) end was closed and the rear end was connected to larger tubes leading to one side of an inclined water gauge. The other side of the gage was left open to the pressure in the room.

The manner of mounting the pressure tube in the 54 -inch wind tunnel is indicated in Fig. 9. The tube $A B$ with the static opening at $C$, was strung through one of the air passages of the radiator and suported by fine wires of hard-drawn brass running over pulleys $D$ and $E$, 3.I cm (I.2.inches) in diameter and held taut by weights. As the tube was moved forward or backward the position of the static pressure opening was indicated by a pointer on the wire traveling over a scale $S$. The pulleys $D$ and $E$ were supported by fine wires, and turnbuckles near the tunnel walls provided a convenient means of adjusting their position. The only important detail in which the mounting in the steam tunnel 
differed from that in the open tunnel was that instead of using wires to support the pulleys $D$ and $E$, cross bars of brass $9.5 \mathrm{~mm}$ $(3 / 8$ inch) in diameter were used to support grooved rings $19 \mathrm{~mm}$ $(3 / 4$ inch $)$ in diameter over which the wires ran. Before taking readings in either tunnel the tube was set so that either the static pressure opening or a point at a known distance from it was at one face of the radiator and the corresponding position of the pointer was noted.

I6. OBSERVATIONS. - The method of observation was to obtain some desired air speed in the wind tunnel and holding that speed as nearly constant as possible, to measure its value and read simultaneousiy the differential pressure on the gage connected to the pressure tube with the opening $C$ in different positions, covering a range from somewhat in front of the front face to a short distance behind the rear face.

Preliminary work in the open tunnel showed that when the forward pulley $D$ was placed far enough from the radiator, so that it did not affect the readings of the pressure tube, it caused errors in the readings of the pitot-static tube used to measure air speed in the tunnel. Accordingly, the pressure tube and pulleys were removed and with a radiator in place a comparison was made between the speed as indicated by the pitot-static tube and the differential pressure between the static opening $F$ and the air outside of the tunnel. In the later tests this static opening was connected both to the gage of the pressure tube and to a second gage the other end of which was open to the air, the pitot-static tube was removed from the tunnel, and speed was computed from the readings of the second gage. Readings with the pressure tube in different cells of the radiators showed that the air flow through the cells increased slightly ( $\mathrm{I}$ per cent) near free edges over that at the center of the section and decreases somewhat (4 per cent) near a side to which a header is attached. These variations are so slight, however, and effect such a small portion of the section that for practical purposes the flow may be regarded as uniform over the face. Slight differences in adjacent tubes, however, make it necessary in most radiators to use the same air cell for comparison in both the steam and open tunnels. The pressure tube was used in the center of an air cell, and it was found by trial that readings could be duplicated if the tube was centered with reasonable care.

Three quantities are measured-position of the opening $C$ with reference to the radiator, air speed, and pressure difference. The 
position is probably correct to about $0.2 \mathrm{~mm}$ (o.or inch) and the air speed is probably within 2 per cent. The greatest error in the pressure difference is probably due to the fact that on account of the shortness of the steam tunnel the forward crossbar is only $77 \mathrm{~cm}$ (3o inches) in front of the radiator and may have some effect on the velocity distribution over the face. The total error, however, is believed to be within 7 per cent, while comparisons between different radiators are probably good within 2 per cent.

The assumption in article I5 that pressure differences within the air tubes are the same in either tunnel for a given flow of air through the tubes is supported by the following facts: (I) In cores that have smooth, round air cells (Figs. 145 and I46) a smooth constant gradient is set up about 2 or $3 \mathrm{~cm}$ from the front face and continues to a point I or $2 \mathrm{~cm}$ from the rear face. (2) This gradient is the same at the same velocity in cores of different depths, as Table I shows.

TABLE 1.-Pressure Gradients as Functions of Depth of Radiator Air Tubes

\begin{tabular}{|r|r|r|r|r|}
\hline \multicolumn{2}{|c|}{ Tube diameter } & \multicolumn{2}{|c|}{ Radiator depth } & \multicolumn{1}{|c|}{$\begin{array}{r}\text { Pressure } \\
\text { gradient }\end{array}$} \\
\cline { 1 - 4 } $\mathrm{cm}$ & Inches & \multicolumn{1}{c|}{$\mathrm{cm}$} & Inches & \\
\hline & & & & \\
\hline .51 & 0.35 & 12.7 & 5 & 0.66 \\
.91 & .36 & 20 & 8 & .67 \\
.91 & .36 & 25 & 10 & .66 \\
.76 & .30 & 10 & 4 & .84 \\
.74 & .29 & 20 & 8 & .85 \\
\hline
\end{tabular}

Both these facts indicate that the gradient set up does not depend on conditions outside the radiator tubes or, in other words, that the end effects die out very speedily.

i 7. Computation. - It was found that to a first approximation the observed pressure differences were proportional to the square of the air flow, and this relation was used to correct for fluctuations in the speed during a test.

The direct result of one set of observations was a curve such as that of Fig. 10, in which pressure readings are plotted against positions in the air tube. If all these curves had been straight lines through the center portion of the radiators, their slopes could have been determined and used to measure the air flow. But since many were quite irregular the following more general method was used. 
Selecting from Fig. Io a part of the curve $A B C$ well within the radiator, such that $B$ is midway between $A$ and $C$ (along the $X$-axis), let

$$
\begin{aligned}
M & =\text { air flow through the whole core, } \\
x & =\text { distance of any point from rear face, } \\
x_{0} & =\text { abscissa of } A, \\
x_{1} & =\text { abscissa of } B, \\
x_{2} & =\text { abscissa of } C, \\
p & =\text { pressure at any point, } \\
m & =\text { air flow constant } \\
M_{0} & =\text { unobstructed air flow in open tunnel, } \\
\rho & =\text { air density, } \\
S & =\text { air speed in open tunnel. }
\end{aligned}
$$

By "unobstructed air flow," $M_{\mathrm{o}}$, is meant the mass of air that would flow in unit time across unit sectional area of the open tunnel if the radiator were not there. Also, let the mean pressure between $A$ and $B$ and between $B$ and $C$ be represented by $P_{1}$ and $P_{2}$, defined by the equations

$$
\begin{aligned}
& P_{1}=\frac{\int_{A}^{B} p d x}{x_{1}-x_{0}} \\
& P_{2}=\frac{\int_{B}^{C} p d x}{x_{2}-x_{1}}
\end{aligned}
$$

and let

$$
Q=P_{2}-P_{1} \text {. }
$$

The air flow constant $m$ is by definition

$$
m=\frac{M}{M_{0}}
$$

For long straight tubes $Q$ varies over a considerable range ${ }^{13}$ as $\rho^{n-1} S^{n}$, and the same relation has been found to be true in the short tubes of the radiators. A number of radiators were used in the steam tunnel at three or more speeds, and the power of the air flow to which the pressure difference $Q$ was proportional is shown in Table 2. The exponent computed from the Lees formula for long tubes of such diameters and at such speeds as are found in radiators was I.73, and that value has been used throughout the computations of air flow constant.

12 Lees, proceedings, Royal Soc. of London, A-9I, I9I4, P. 46. 
TABLE 2.-Exponent of Air Flow to Which Pressure Drop in the Air Tubes is Proportional

\begin{tabular}{|c|c|c|c|c|c|}
\hline $\begin{array}{c}\text { Radi- } \\
\text { ator No. }\end{array}$ & Type & $\begin{array}{l}\text { Expo- } \\
\text { nent n }\end{array}$ & $\begin{array}{l}\text { Radi- } \\
\text { ator No. }\end{array}$ & Type & $\begin{array}{l}\text { Expo- } \\
\text { nent n }\end{array}$ \\
\hline$A-7 \ldots$ & Square cell.............. & 1.6 & B-17... & Hezagonal cell. ............... & 1.7 \\
\hline$A-9 \ldots$. & .....do............................ & 1.7 & $\mathrm{C}-2 \ldots$ & Pseudo circular cell. . . . . . . . . . & 1.7 \\
\hline$A-14 \ldots$ & Square cell, irregular............ & 1.7 & $\mathrm{C}-12 \ldots$ & True circular cell ............... & 1.8 \\
\hline A-19... & Square cell, walls swaged....... & 1.8 & $\mathrm{C}-13 .$. & 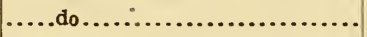 & 1.7 \\
\hline A-29... & Square cell.................. & 1.8 & D-1.... & Irregular. ....................... & 1.6 \\
\hline$A-31 \ldots$ & 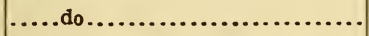 & 1.9 & D-2 .... & .....do..................... & 1.9 \\
\hline $\mathbf{B}-3 \ldots$ & Hexagonal-pseudo cellular.... & 1.8 & D-3... & .....do...... & 1.5 \\
\hline $\mathbf{B}-13 \ldots$ & 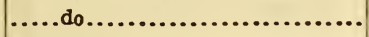 & 1.6 & & $\cdot$ & \\
\hline
\end{tabular}

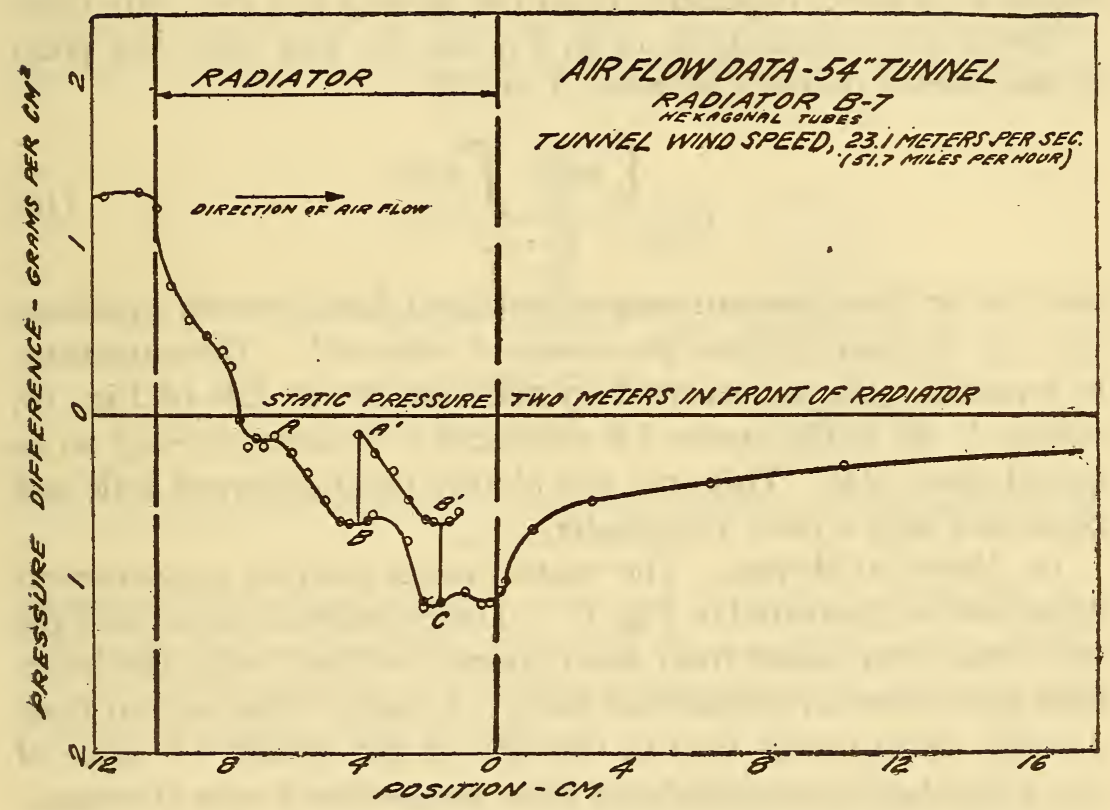

Fig. I0.-Pressure drop in the air tube of a radiator with irregular shaped cells

The displaced segment of the curve $A^{\prime} B^{\prime}$ is for measurement of air flow under conditions where the pressure gradient is not linear with distance along the tube axis. Method explained in Art. I 7

The pressure difference $Q$ may be expressed by the equation

$$
Q=b^{\prime} \rho^{\mathrm{n}-1} S^{\mathrm{n}}
$$

which, since

$$
M=\rho S
$$

may be written

$$
Q=b^{\prime} \frac{M^{\mathrm{n}}}{\rho} .
$$

But the density was nearly the same in both tunnels, and the small differences existing were negligible in the result. ${ }^{13}$ The

18 See Appendix C. 
density was accordingly combined with the constant $b^{\prime}$ and equation (6) written

$$
Q=b M^{\mathrm{n}}
$$

In the closed tunnel $Q$ and $M$ were measured at three or more speeds and a mean value of $b$ obtained. In the open tunnel

$$
M=\left(\frac{Q}{b}\right)^{\frac{1}{n}}
$$

and

$$
M_{\mathrm{o}}=c S \text {, }
$$

where $c=a$ factor depending upon the density and the units used.

Now, by the definitions of $Q, P_{1}$, and $P_{2}$, and since the point $B$ was chosen midway between $A$ and $\mathrm{C}$,

$$
Q=\frac{\int_{B}^{C} p d x-\int_{A}^{B} p d x}{x_{1}-x_{0}}
$$

and the air flow constant may be obtained directly from equations (3), (9), (8), and (Io) and the observed value of $b$. The numerator of equation (Io) is measured by the area $A^{\prime} B^{\prime} C B$ of Fig. Io, where $A^{\prime} B^{\prime}$ is the curve $A B$ displaced a distance $\left(x_{1}-x_{0}\right)$ so as to fall above $A B$. This area was plotted on an enlarged scale and measured with a polar planimeter.

I8. VENTURI METER.-The venturi meter used for measurement of air flow is illustrated in Fig. I I. The cylindrical throat and the two cones were made from sheet copper soldered, with the joints filed into curves of stream-line form. A copper tube led out from a single small needle hole in the side of the throat. A piece of sheet iron bent into cylindrical form was soldered over the cones, extending from in front of the front cone nearly to the rear end and covering the central contracted portion which otherwise would cause considerable resistance to the air stream. A second needle hole in front of the front cone was connected to a small tube which ran back along the outer surface, turning up where it reached the tube coming from the throat. The instrument was $25 \mathrm{~cm}$ (Io inches) long, with a mouth $3.5 \mathrm{~cm}$ (I $3 / 8$ ) inches in diameter. Three soft iron wires soldered to the sides of the instrument were passed through cells of the radiator and bent over in front to hold it in place on the rear face. The pressure difference was measured on an inclined water gage.

The meter was attached to the rear face of a radiator placed in the steam tunnel and a relation found between the pressure differ- 
ence reading of the meter and the air flow through the core. It was then used similarly on the same radiator in the open tunnel and a relation found between its pressure difference and the tunnel speed (corresponding to flying speed). From these two relations air flow in the core was determined in terms of flying speed, on the assumption that the venturi pressure difference was the same in both tunnels for a given air flow through the core. The method evidently amounts to a calibration of the meter with each radiator on which it was used, but was necessary because the meter readings in the steam tunnel varied with different radiators for the same air flow.

In a few cases the air flow constant was determined both with the venturi meter and with the pressure tube, and a fair agreement

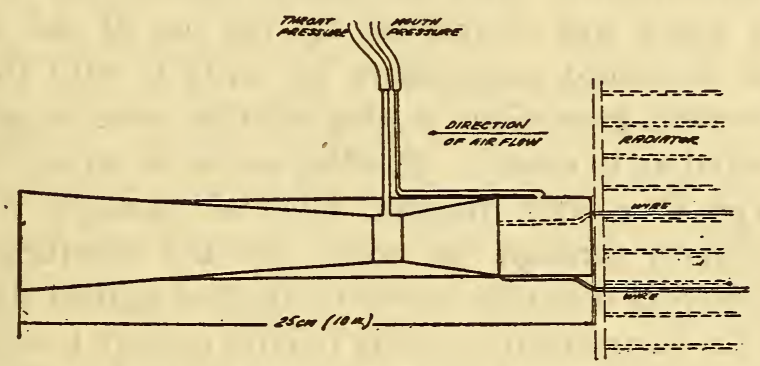

FIG. II.-Venturi meter for comparison of air flows through the core of a radiator under various conditions

between the two instruments is indicated in Table 3. Throughout this paper the constant determined by the use of the pressure tube has been used wherever possible for computations, since that seems to be more reliable than the other.

TABLE 3.-Air Flow Constants by Venturi Meter and by Pressure Tube

\begin{tabular}{|c|c|c|c|c|c|}
\hline Radiator No. & By venturi & $\begin{array}{l}\text { By pressure } \\
\text { tube }\end{array}$ & Radiator No. & By venturi & $\begin{array}{l}\text { By pressure } \\
\text { tube }\end{array}$ \\
\hline$A-6 \ldots . . . . . . . . .$. & 0.59 & 0.53 & B-8................ & 0.52 & 0.44 \\
\hline$A-13 \ldots \ldots, \ldots, \ldots$ & .55 & .57 & $C-1 \ldots . . . . . . .$. & .45 & .43 \\
\hline$A-21 . . . . . . . . . . . .$. & .51 & .48 & c-4...................... & .62 & .50 \\
\hline$\dot{A}-23 . . . . . . . . . . . . . . . .$. & .51 & .49 & C-7...................... & .60 & .61 \\
\hline B-1....................... & .71 & .68 & & & \\
\hline
\end{tabular}

B. AIR FLOW FOR UNOBSTRUCTED POSITIONS OF RADIATOR

I9. SPEed And Density.-With the possible exception of certain types of radiator mentioned in the next paragraph, the air flow through the core is proportional to the speed of flight and to the density of the air when unaffected by other parts of the craft 
on which the radiator is mounted; $i$. e., when the radiator is in an unobstructed position. This relation may be expressed by the equation

where

$$
M=m \rho S^{\prime},
$$

$$
\begin{aligned}
M & =\text { air flow, mass per unit time per unit frontal area, } \\
\rho & =\text { density, } \\
S^{\prime} & =\text { flying speed } \\
m & =\text { "air flow constant." }
\end{aligned}
$$

If consistent units are chosen for $M, \rho$, and $\mathrm{S}^{\prime}$, the constant $m$ will be dimensionless and is, as stated above (art. 4), the fractional part of the air directly approaching the core that passes through it, or unity minus the fraction deflected around it.

In the experimental work the linear relation between air flow and flying speed was established by the use of the air venturi meter, and it seemed unnecessary to verify it with the pressure tube. Apparent exceptions to this relation were found for cores so constructed as to cause a whistling sound in an air stream and for one type, $\mathrm{G}-4$, with irregular sided air passages which carry the air obliquely through the core. For the whistling types the venturi gave very irregular curves of air flow against flying speed, and while the instrument is hardly reliable enough to warrant very much confidence in the curves, it is probable that some irregularity exists, because the curves of head resistance against flying speed also show unusual form (art. 38). For the type $\mathrm{G}-4$ the venturi gave a linear relation over the range 8 to $33 \mathrm{~m}$ per sec ( 15 to 75 miles per hour), but the line if produced would have indicated zero air flow at about $3.5 \mathrm{~m}$ per sec ( 8 miles per hour). But little confidence should be placed in the measurement of air flow in this type or in the whistling types.

20. Empirical EQUATION FOR AIr Flow Constant.-The air flow constant $m$ is a function of the geometrical form of the radiator and of the nature of the cooling surfaces, and for the more common types ranges from 0.4 to 0.7 and has been found as low as 0.3. Its values for about 80 types of core are given in Table 21 . The air flow constant may be expected to be a function of the "free area," the depth, and the hydraulic radius of the air tubes. Since $m$ is dimensionless, the depth and the hydraulic radius, each having the dimension of a length, must enter the function as a ratio, and $m$ may be of the form

$$
m=f\left(a, \frac{r}{x}\right)
$$


Also

$m=\mathrm{o}$ when $a=\mathrm{o}$, and when $r=\mathrm{o}$

and

$m=\mathrm{I}$ when $a=\mathrm{I}$ and $x=\mathrm{o}$

where

$a=$ free area of radiator,

$x=$ depth of radiator,

$r=$ hydraulic radius of air tubes.

These conditions are satisfied and the experimental values fairly well represented by the empirical equation

$$
m=a\left(\mathrm{I}-\mathrm{IO}^{-\frac{\mathrm{k}}{\mathrm{x}}}\right)
$$

where $k$ is a constant. If the constant $k$ is computed from measurements on a particular type of core, the equation may be used for a good estimate of air flow through a similar type of different depth, and a fair estimate may be made for any ordinary type of cellular core by using a value of 44 for $k$. The degree to which this value represents actual values is indicated in Table 4 , which compares observed air flow constants with those computed by means of the equation using $k=44$. The value of $k$ depends to some extent upon the form of the air tubes, including the ends of the tubes, and upon the smoothness of the surface, but does not vary very widely for the different types tested. The equation is of proper form to represent such conditions as that the air flow increases with increase in free area and in hydraulic radius of air passages, and decreases with increase in depth, which adds to the frictional resistance.

21. Nature of Air-Tube Walls.-The air flow is, of course, greater when the cooling surfaces are smooth than when they are of the same dimensions but rough, though the surfaces commonly found in well-made radiators do not differ enough in smoothness to show any marked effect. The section $\mathrm{C}-8$, circular tubes with hexagonal ends, as received from the manufacturer had very rough surfaces, and its air flow was estimated from measurements with the venturi meter both as received and after the tubes had been somewhat smoothed. The difference in nature of the surfaces resulted in an increase of 5 per cent in the air flow, but this increase is greater than would ordinarily be obtained by polishing the tubes of a radiator, because the original surface was unusually rough. For large air flow the walls of the air passages should be not only smooth but straight. Projections, indentations, and holes in the walls cause additional resistance and thereby reduce the air flow. 
TABLE 4.-Comparison of Observed Air Flow Constants With Values Computed from Empirical Relation $m=a\left(1-10^{-44} \mathrm{r} / \mathrm{x}\right)$

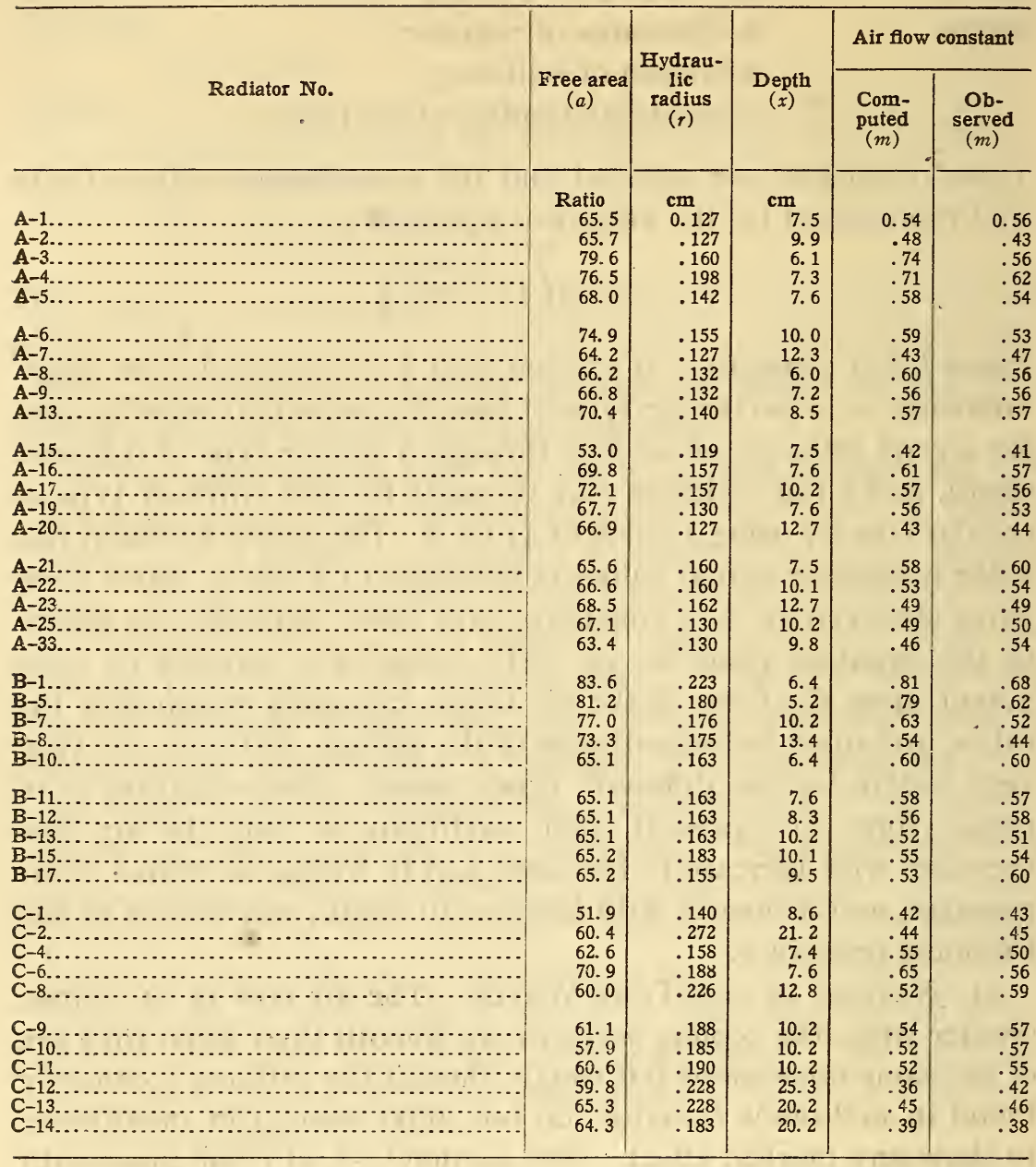

22. YAW.-The effect of yawing the radiator or turning it so that the air strikes it at some other angle than normal to its face is to decrease the flow of air. No quantitative relation between angle of yaw and decrease in air flow can be given, however, because of the lack of reliable measurements, and the effect is doubtless considerably greater with some types of core than with others.

\section{AIR FLOW FOR OBSTRUCTED AND SLIP-STREAM POSITIONS}

Only a very limited study has been made of obstructed and slip-stream positions because of experimental difficulties and the lack of facilities for tests on full-size airplanes, and the results of 
the experimental work for such positions must be regarded as qualitative only.

23. NOSE OF THE FUSELAGE.-In order to study the properties of a radiator mounted in the nose of a fuselage, a rough model of a fuselage was constructed. The model (illustrated in Figs. I2 and $\mathrm{r} 3$ ) was about $1.5 \mathrm{~m}$ (6o inches) long, $25 \mathrm{~cm}$ (Io inches) wide, and $33 \mathrm{~cm}$ ( 13 inches) high, and was made to accommodate a $20 \mathrm{~cm}$ (8 inch) square section of core in the front, which could also be covered by a detachable stream-line nose. Two rectangular openings, each about 3.8 by $16.5 \mathrm{~cm}$ ( $1 \mathrm{r} / 2$ by $6 \mathrm{I} / 2$ inches), were cut in each side of the body, a little behind the position of the radiator, and were fitted with adjustable sliding doors, which allowed some control of the amount of air passing through the radiator. The model was mounted in the 54-inch wind tunnel in the same manner as a radiator, and the air venturi meter was attached to the radiator inside of the body. The values obtained for the air flow constant were as follows, the normal air flow constant (for unobstructed positions) being given for comparison:

TABLE 5.-Estimated Air Flow Constants, Radiator in Nose of Fuselage

[The figures given are those obtained in measurements made with a model shaped like a fuselage but much smaller and without engine, etc.]

\begin{tabular}{|c|c|c|c|c|}
\hline $\begin{array}{c}\text { Radiator } \\
\text { No. }\end{array}$ & + & $\begin{array}{l}\text { Vents } \\
\text { closed }\end{array}$ & $\begin{array}{l}\text { Vents } \\
\text { open }\end{array}$ & $\begin{array}{l}\text { Unob- } \\
\text { structed } \\
\text { position }\end{array}$ \\
\hline A-23.. & Square cell................. & 0.15 & 0.36 & 0.49 \\
\hline$B-1 \ldots .$. & Hezagonal, pseudocellu lar..... & .15 & .38 & .68 \\
\hline$G-3 \ldots \ldots$ & 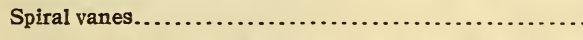 & .15 & .32 & .37 \\
\hline
\end{tabular}

The great reduction in air flow constant furnishes a good illustration of the effect of obstruction in the air stream behind the radiator. Although in actual airplanes louvres on the sides of the fuselage may tend toward an increase in air flow, the model contained nothing corresponding to the engine which offers a considerable obstruction to air flow in actual installations.

It may be stated that, in general, the air flow through a radiator in the nose of a fuselage is much less than for the same type of core in an unobstructed position, and it appears likely that for common types of core mounted in the nose the air flow constant falls between 0.1 and 0.4 , or perhaps 0.5 , depending upon the type of core, the obstructions behind the radiator, and the facilities provided for letting the air out of the fuselage.

24. Wing Positron.-While the air flow through a radiator in the nose of a fuselage is low, it is probably much lower through 
a radiator mounted in the wing, so that its faces conform approximately to the upper and lower surfaces of the wing. For a rough estimate of air flow a half-size section of wing was used, 66 by $107 \mathrm{~cm}$ (26 inches wide by. 42 inches deep), of the U. S. Navy X type, with the fabric cut away to accommodate a section of radiator. The radiator core was 25 by $4 \mathrm{I} \mathrm{cm}$ (ro by 16 inches), with $8 \mathrm{~mm}$ ( $\frac{5}{16}$ inch) square tubes $8.5 \mathrm{~cm}$ ( $31 / 2$ inches) long, sloping at an angle of $45^{\circ}$ upward and to the rear. The wing and radiator were mounted in the 54-inch wind tunnel in such a way that the angle of attack could be set at will.

Because of the change in direction of the air stream both on entering the radiator and on emerging from it the usual methods could not be used for measuring air flow and an estimate was made by the following method: One of the air tubes near the center of the core was plugged with putty, and two small copper tubes with dynamic pressure openings were placed inside of the plugged tube, facing the plug from opposite sides of the core. Readings were taken of the pressure difference between these dynamic openings and of the speed of the stream in the tunnel, and in order to show the meaning of the readings an ordinary square. cell radiator of the same diameter and length of cell was used in a similar manner in the steam tunnel, where the air flow through it could be measured. By this means a relation was found between the air flow and the pressure difference obtained by the opposed dynamic pressure openings in the plugged tube. The estimated air flow constants obtained by this method were as follows:

TABLE 6.-Estimated Air Flow Constants in Wing Radiator

\begin{tabular}{|r|r|}
\hline Angle of attack & $\begin{array}{r}\text { Air flow } \\
\text { constant }\end{array}$ \\
\hline $2^{\circ} 15^{\prime} \ldots \ldots \ldots \ldots . \ldots$ & 0.16 \\
$4^{\circ} \ldots \ldots \ldots \ldots \ldots$ & .18 \\
$8^{\circ} 15^{\prime} \ldots \ldots \ldots \ldots$ & .26 \\
\hline
\end{tabular}

For unobst ructed position, constant $=0.55$

25. Slip-Stream Positions.-If the blast from the propeller blows over the radiator, the air flow is increased by an amount that depends upon the distance from the propeller, the position along a radius of the slip stream, and the "slip coefficient" of the propeller. Many designers assume that the relative speed of the air is greater in the slip stream than out of it by a definite amount, and the figures assumed for the increase range from io to 25 per cent, but such an assumption can not be generally true. 

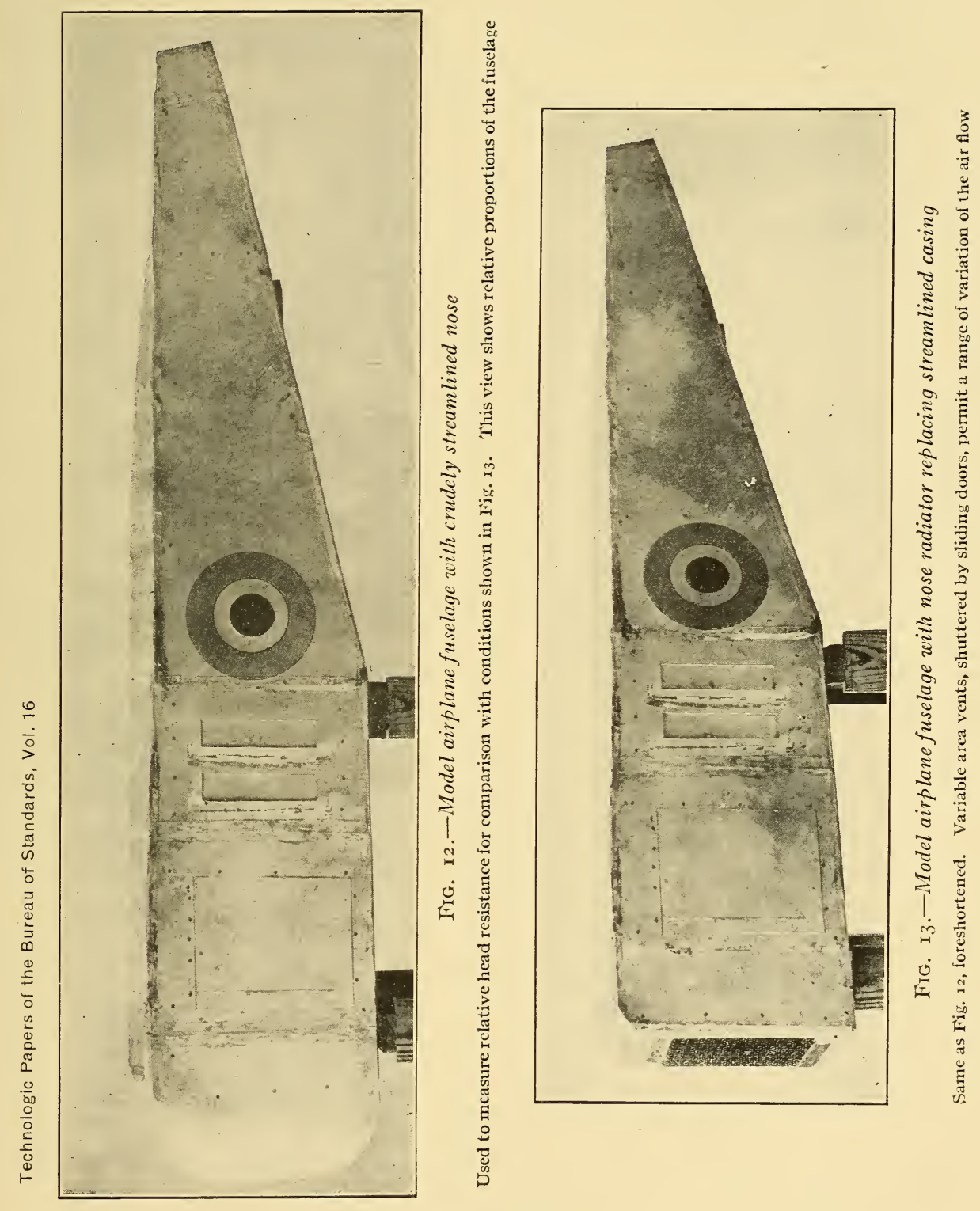

If, however, the relative air speed is, for example, 20 per cent greater in a certain part of the slip stream than out of it, the air flow through a radiator in such a position will not be 20 per cent greater than it would be but for the effect of the propeller because of the swirl in the slip stream, which makes the air strike the radiator at an angle with its face, and is equivalent to yawing the radiator or turning it through an angle. ${ }^{14}$

\section{HEAD RESISTANCE AND POWER ABSORBED}

26. General Statement.-The head resistance of a radiator is closely related to the air flow through it, for the factors which cause high resistance also cause the air flow to be low. The general trend of the relation between head resistance and air flow is indicated by the line on Fig. 14, which shows head resistance factor in terms of air flow constant. The scattering of the points is probablydue largely to differences in form of the ends of the air tubes.

Since some air passes through the radiator and some is deflected around it, the effect of flow through tubes is combined with the effect of a solid body in an air stream, and the head resistance is accordingly made up

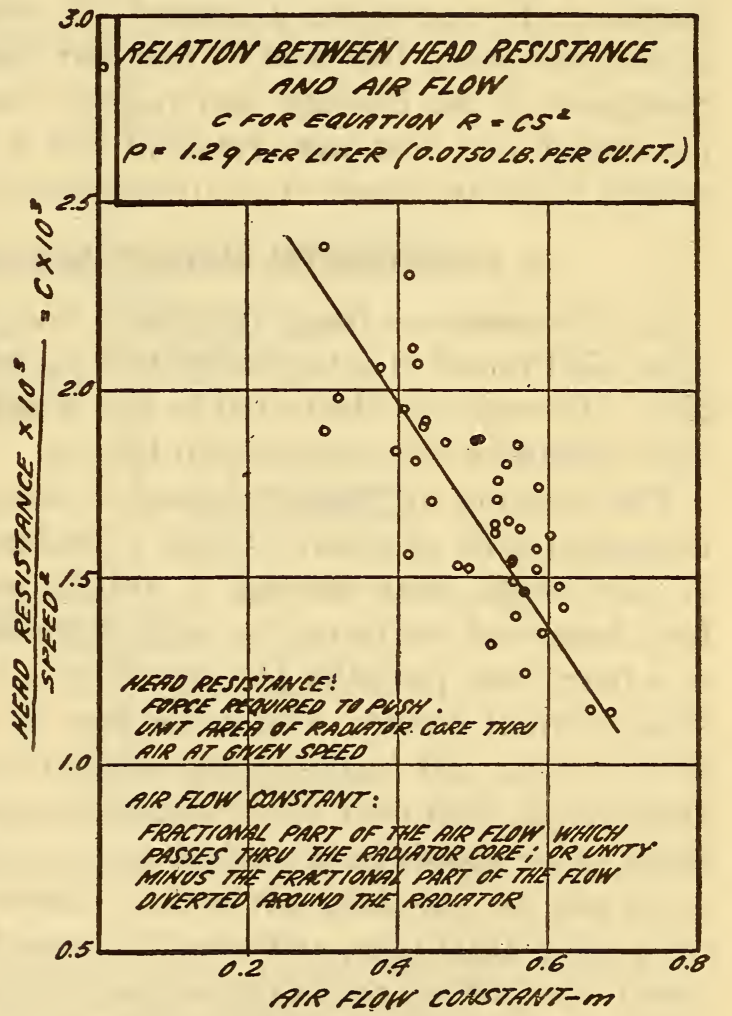

FIG. I4.-Relation between head resistance of a section of radiator core and air flow through the core

Shows the larger head resistance for radiators of design such as to pass but a small fraction of the air stream through the core, deflecting the major fraction around the radiator of two parts, one due to actual surface friction on the walls of the air passages and the other to the action of the radiator in dis-

\footnotetext{
${ }^{14}$ For a discussion of effects of the slip stream the reader is referred to Technical Report No. $7 x$ of the National Advisory Committee for Aeronautics, "Slip-Stream Corrections in Performance Computation."
} 
turbing the flow of air. The second part corresponds to the resistance of solid bodies and is usually accompanied by eddies in the air stream. For convenience this kind of resistance will be called "eddy resistance." Eddy resistance is found not only at the entrance and exit of the air passages, but at any points where there are projections, indentations, or holes, in the air-tube walls.

If the radiator is in an obstructed position, it may so modify the flow of air around other parts of the craft on which it is mounted as to increase the total resistance of the craft by an amount considerably greater than its own resistance when in an unobstructed position. For example, if instead of using a fuselage with a stream-line nose, the nose is flat and contains a radiator, the resistance of the fuselage and radiator may be greater than if the stream-line nose were retained and a radiator of equivalent cooling capacity placed in an unobstructed position.

\section{A. EXPERIMENTAL, METHOD AND COMPUTATION}

27. Apparatus.-Head resistance was measured in the large open wind tunnel of octagonal section 54 inches $(137 \mathrm{~cm})$ in diameter. The tunnel is illustrated in Fig. 8 and the balance on which head resistance was measured in Fig. I5.

The entering air passes through a honeycomb of sheet-metal hexagonal cells of about $75 \mathrm{~mm}$ (3 inches) diameter and $30 \mathrm{~cm}$ (I foot) depth, then through a straight section about $7.6 \mathrm{~m} \mathrm{(25}$ feet) long, and out through a conical diffuser, at the end of which is a four-blade propeller fan driven by a $55 \mathrm{kw}$ ( 75 horsepower) direct-current motor. The motor may be operated on either I 20 or 240 volts, and further speed control is obtained by means of rheostats in both field and armature circuits, giving a continuous range of air speeds from $7 \mathrm{~m}$ per sec. ( 5 miles per hour) to about $40 \mathrm{~m}$ per sec. (90 miles per hour). The air speed was measured by a pitot-static tube, and when the tunnel was unobstructed the speed was uniform across the section to about I per cent.

The balance on which head resistance was measured is illustrated diagrammatically in Fig. 15. It consists essentially of a bell crank suspended by thin flexible steel strips (safety razor blades). An adjustable counterpoise balances the weight of the horizontal arm, while heavy weights just above the damping vane lower the center of gravity enough to insure stability when using a heavy section for test. The vertical arm supporting the section is extensible and is fitted at the top with a detachable brass block, 
which is screwed to a plate not more than $3 \mathrm{~mm}$ ( $1 / 8$ inch) thick, soldered to the lower side of the radiator.

The balance measures the amount of the head resistance, and in order to compute the actual force exerted on the radiator it is necessary to know the ratio of the distances from the fulcrum to the point on the horizontal arm where the weights are hung and to the center of pressure on the radiator. Since it was not convenient to measure these distances with accuracy, their ratio was checked by a calibration of the balance, made by applying known

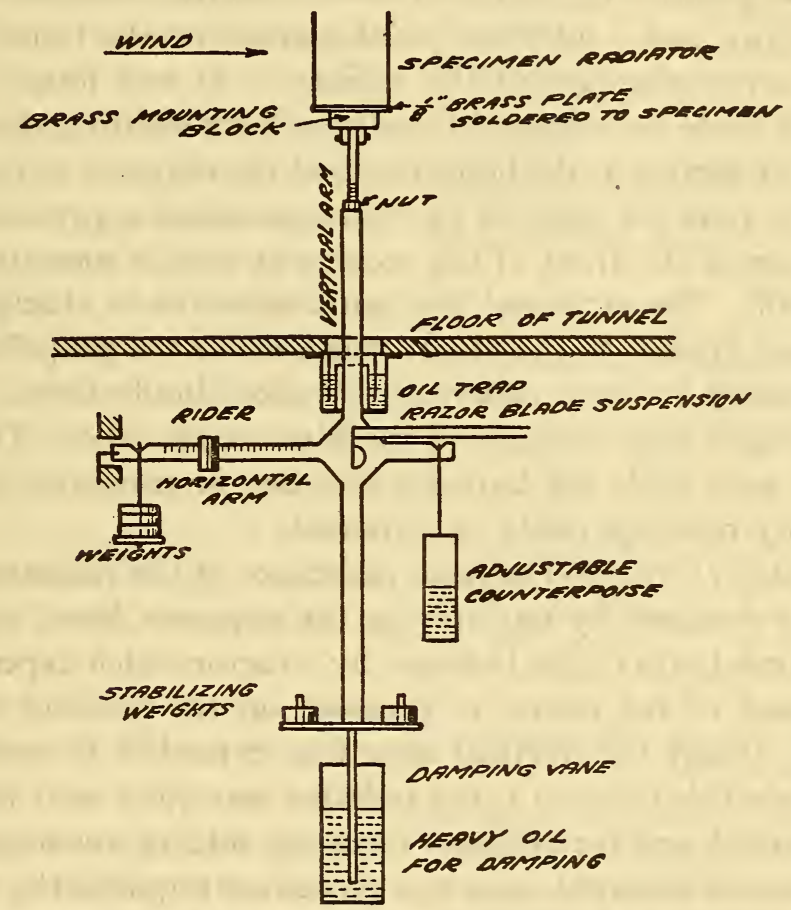

FIG. 15.-Aerodynamic balance for measurement of head resistance of a section of radiator core mounted in large wind tunnel

horizontal forces to the vertical arm. Readings were taken with the vertical arm extended to three different lengths.

Preliminary investigations showed that ordinary radiators as manufactured are sufficiently uniform that the center of pressure of a section of core may be assumed to lie at the geometrical center, and that the resistance is the same with either face presented to the wind. (This is not true of certain special types whose front and rear faces are not similar.) As stated above (art. 13), the disturbance of the air stream by the radiator seemed 
to be confined well within the section of the tunnel, so that openair conditions were well represented.

28. OBSERVATIONS. - The method of taking readings was as follows: The radiator (bare core, without headers) was mounted on the vertical arm of the balance and the distance measured from its center to a fixed point on the arm. The distance from this point to the fulcrum was determined by the calibration mentioned above. To determine the position when the radiator was normal to the air stream (no yaw), a section was set at various angles, the position of minimum head resistance assumed to represent zero yaw and a reference point marked on the tunnel wall by sighting across the face of the radiator. It was found that the alignment could be obtained equally well by sighting through the tubes of the section at the honeycomb at the entrance of the tunnel. For yawed runs the angle of yaw was measured approximately by sighting across the front of the section at a scale mounted on the tunnel wall. The air speed was quite sensitive to changes in the line voltage applied to the motor which drove the propeller, and it was necessary for two observers to take simultaneous readings on the balance and the gage of the pitot-static tube. There was, however, very little lag between the two instruments, and very satisfactory readings could be obtained.

29. Computation.-The head resistance of the radiator and the support is obtained by multiplying the apparent force, as read on the horizontal arm of the balance, by a factor which depends upon the distance of the center of pressure on the radiator from the fulcrum. When the vertical arm was extended to such length which made this factor 0.5 , the radiator was quite near the center of the channel, and for ordinary tests this setting was used. With test sections of different sizes this procedure required the exposure of different lengths of the supporting arm to the air stream.

To correct for the resistance of the supporting arm, its resistance was measured at various speeds when extended to three different lengths, and a quantity corresponding to the length used was subtracted from the apparent resistance of the radiator as read on the balance. Because of the mutual effect of the radiator and its support the resistance of the combination is probably not exactly equal to the sum of the resistance of the parts when alone and the procedure doubtless introduces a small error. Since, however, the correction for the supporting arm was usually but 7 to 12 per cent of the resistance of the radiator, the errors in this 
correction are probably well within the limit set by other conditions in the experimental work.

Head resistance was expressed in pounds and reduced to I square foot frontal area and to a "standard" air density of 0.0750 pounds per cubic foot $\left(\mathrm{I} .204 \mathrm{~kg} / \mathrm{m}^{3}\right)$. For this reduction it was assumed, in accordance with aerodynamic practice and the results of observations on pressure drop through radiator sections in the closed tunnels, that head resistance is proportional to density. The tests showed that the resistance varies roughly as the square of the speed, and within the range of sizes used the resistance per unit frontal area (which is the meaning of the term "resistance" as used throughout this report) is not appreciably affected by size or shape. The departure of the resistance from variation as the square of the speed is indicated in Table 8 (art. 35). The effect of size and shape is shown in Fig. I6. The sizes used ranged from $20 \mathrm{~cm}$ by $20 \mathrm{~cm}$ ( 8 by 8 inches) to $40 \mathrm{~cm}$ by $40 \mathrm{~cm}$ ( 16 by I 6 inches) and $30 \mathrm{~cm}$ by $60 \mathrm{~cm}$ (12 by 24 inches).

30. Reduction for AIR DenSITY.-The reduction of head resistance to the value it would have in air of standard density may be accomplished by merely substituting standard for actual

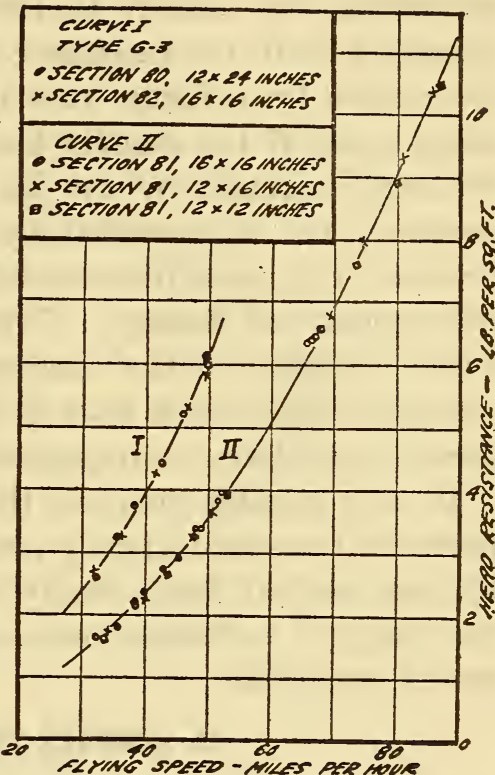

FIG. I6.-Head resistance per unit area of sections of core differing in shape and size but otherwise exactly alike. No variation with size discernible density in the computation of air speed, because a change in density has similar effects upon the resistance of the radiator and upon the pressure difference impressed on the pitot tube used to measure the air speed. If

$R=$ head resistance per unit area,

$S=$ speed of the air stream,

$\rho=$ air density,

$x=$ differential pressure of the pitot tube, $a, b, b^{\prime}=$ constants, 
the resistance is given by an equation of the form

$$
R=a \rho S^{2}
$$

and the equation of the pitot tube

may be put in the similar form

$$
S=b^{\prime} \sqrt{\frac{x}{\rho}}
$$

$$
x=b \rho S^{2} .
$$

From these equations it will be evident that if during the observations the density is, for example, 2 per cent greater than standard, both the resistance $R$ and the pressure difference $x$ will be greater by a factor 1.02 than they would have been for the same speed if the density had been standard; but while the resistance is greater by the factor I.02, the speed computed from equation (2) is increased by a factor $\sqrt{ } \mathrm{I} .02$, and the relation between the quantities actually measured, namely, $R$ and $x$, is independent of density. Thus, the use of standard rather than actual density for the computation of speed, results in giving a value for speed such that if the density had been standard this speed would have corresponded to the observed resistance.

If, as is usually the case, the resistance is proportional to some power of the speed slightly less than 2 , a small error is introduced by this method, but a study of its effects has shown that within the range of variations encountered in work with radiators the error is negligible.

\section{B. SURFACE FRICTION IN AIR TUBES}

31. Radiators Not Similar to Long Tubes. - The laws of surface friction in long circular tubes have been studied by many investigators, and the formula of $\mathrm{C}$. H. Lees ${ }^{{ }^{15}}$ represents the facts over a very wide range of diameters of tubes and of speed, density, and viscosity of fluid, but only for a middle part of the tube, so far from the ends that end effects may be neglected. Of the exact behavior of air at the ends of the tubes, however, and of the energy transformations involved at the ends much less is known. The minimum distance from either end of a tube at which many investigators would have confidence in measurements of static pressure is considerably greater than the total length of ordinary radiator tubes, and the laws of flow in long tubes can be applied to radiators only with great caution. Nevertheless, it should be borne in mind that in a cluster of tubes such as a radiator conditions correspond-

${ }^{16}$ Proceedings, Royal Soc. of London, A-9r, I914, p. 46. 
ing to long tubes may be found much nearer the ends than in a single tube. The very divergent flow lines characteristic of entrance and exit for a single tube are doubtless found true for tubes near the edge of a radiator, but except near the edges it is likely that the mutual interactions of the multitude of streams must necessarily result in more nearly parallel flow lines even before entering the tubes. A further caution in applying deductions from flow in long, circular tubes is to bear in mind that while some radiators are constructed with circular air tubes of fairly smooth walls others have square, hexagonal, elliptical, and irregular shaped cells, while the "fin and tube" types can not be said to have air tubes, and many of the so-called cellular types do not have true air tubes but merely "passages" more or less cut up by projecting strips of metal, known as "fins." Variations in surface friction are also indicated by differences in kind or degree of turbulence in different radiators (Appendix B).

Figs. 138 to 152 , showing pressure in the air tubes as indicated by the pressure tube used in measuring air flow, give some indication of the effects of surface friction and of the relations between surface friction and total head resistance; i. e., surface friction plus "eddy resistances" at the faces of the radiator. The plots show clearly the contraction of the jets of air on entering the tubes and their expansion after leaving the radiator, and the differences in form of the curves give some qualitative indication of the behavior of the air at the ends of the tubes, though measurement of static pressure is difficult under such conditions and great significance should not be attached to the exact form of the curves drawn.

32. Comparison of Pressure Gradients in Radiators With VALUES FOR LONG TUBES. - In view of the preceding statements, it is hardly to be expected that a simple relation would be found between the surface friction in ordinary radiator tubes, their dimensions, and the speed and other properties of the air; nor is it surprising that in the case of types with circular cells, in which a pressure gradient may be measured by the use of the pressure tube, this gradient should not agree closely with that computed by means of the Lees ${ }^{16}$ formula. The gradients meas-

\footnotetext{
16 The Lees formula is as follows, absolute units in any consistent system being used throughout: 
ured in five radiators of circular tube types were found to exceed those computed by the formula for similar conditions by the amounts shown in Table 7 .

TABLE 7.-Excess of Observed Over Computed Pressure Gradients in Air Tubes

\begin{tabular}{|c|c|c|}
\hline t & Radiator No. & Excess \\
\hline & $\ldots$ & Per cent \\
\hline$C-11 .$. & 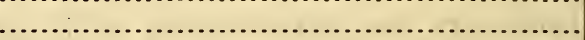 & $\begin{array}{r}0 \\
19\end{array}$ \\
\hline$C-12 \ldots$ & & 30 \\
\hline$C-13 \ldots$ & & 31 \\
\hline$C-14 \ldots$ & & 1 \\
\hline
\end{tabular}

But, although the observed values of surface friction were higher than those computed by the formula, the powers of the air flow to which the friction is proportional agree fairly well with the formula, as is shown by Table I (art. I7).

33. Condition of Surface.-The effect of roughness of surface is to increase surface friction, but the surfaces usually found in radiators do not differ enough in roughness to show any very marked effect. This effect was studied by two methods-by measuring the pressure gradient in a single brass tube with different conditions of surface, and by comparison of the gradients obtained with the pressure tube in two radiators whose air tubes were of nearly the same form and dimensions but with quite different conditions of surface.

The single tube was $105 \mathrm{~cm}$ (4I.3 inches) in length, with an inside diameter of $0.95 \mathrm{~cm}(3 / \mathrm{s}$ inch). Small holes were drilled in it at $10 \mathrm{~cm}$ intervals, beginning $5 \mathrm{~cm}$ from each end, and tubes were attached to give static pressure at each of these I I positions. Since it was necessary to remove the burr from the inside of the tube after the holes were drilled, the original surface was not used, and the first measurements were made with the tube somewhat polished. Other measurements were made with the surface oiled lightly, smoked lightly, and roughened somewhat. The air flow was measured by a special Thomas meter made for the purpose. Although it was not calibrated for lack of convenient apparatus, the meter was made with some care, and was doubtless good at least for comparative purposes. Pressure gradients were obtained by plotting the pressures read at the II static-pressure holes against their respective positions and were expressed in grams per square centimeter per centimeter length of tube. The 
observations on the smoothed and oiled surfaces were very consistent, but when the tube was smoked or roughened they were less consistent, probably because of effects of the smoking and roughening on the static-pressure openings. The errors due to these irregularities, however, do not exceed 3 per cent. Corrections for the effect of changing density of the air were of the order of I per cent and were neglected.

No differences in pressure gradient obtained with the different surfaces were found to be outside the probable limit of experimental error, with the possible exception of the smoked surface, which would probably have shown a greater difference if the tube had been more thoroughly smoked. The length and small diameter of the tube made roughening the surface somewhat inconvenient, and the pressure gradient would, without doubt, have been considerably increased had the surface been made considerably more rough, this being indicated by the results of measurements in a tube of a radiator. The results obtained are shown in Fig. I7.

The two radiators used for comparison were the sections $\mathrm{C}-9$ and $\mathrm{C}-\mathrm{ro}$, which were very nearly alike except that the tubes of $\mathrm{C}$-ro were very rough,

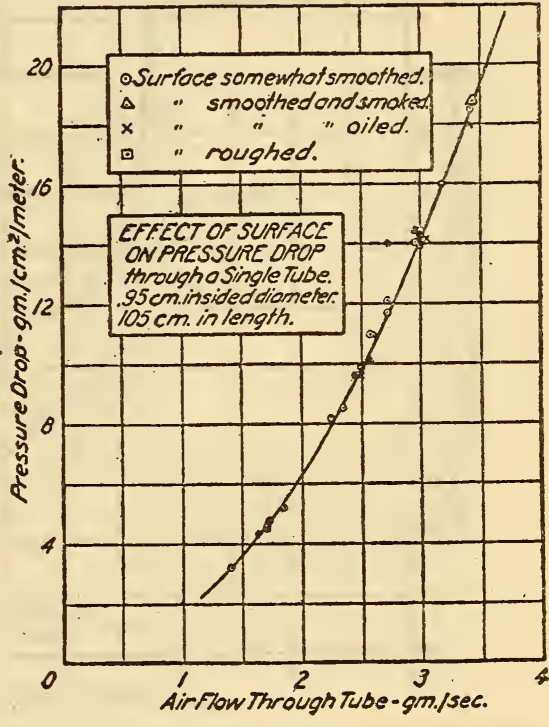

Fig. 17.-Effect of condition of surface of an air tube on surface friction.

Four different conditions of surface with all other factors constant show no significant differences in pressure gradient

while those of $\mathrm{C}-9$, though originally in the same condition, had been somewhat smoothed. Observations were made at three rates of air flow, two of which are shown in Fig. I8, and the gradient in the rough tube was found to be greater than that in the smoother tube by 22 per cent. When reduced, however, to the same linear speed through the tubes (by means of the ratio of the "free areas" of the cores and the assumption that the pressure gradient varies as the 1.7 power of the speed) the difference becomes 12 per cent. ${ }^{17}$

\footnotetext{
17 While for some types of core there is considerable variation between individual air tubes, these types show very good uniformity, and this figure is probably good for the core as a whole.
} 
34. EFFECT OF HEAT IN THE RAdIATOR.-The surface friction in the air tubes also depends upon the temperature difference between the air and the radiator. The pressure gradient in the type $\mathrm{C}-9$ was measured both when the radiator was cold and when heat was supplied, as in calorimetric tests, and the gradient was found to be about i 5 per cent higher when hot than for the same air flow when cold, with a mean temperature difference of $6 \mathrm{I}^{\circ} \mathrm{C}\left(\mathrm{IIO}^{\circ} \mathrm{F}\right)$ between the water in the radiator and the entering air. This comparison is shown for three speeds in Fig. I9.

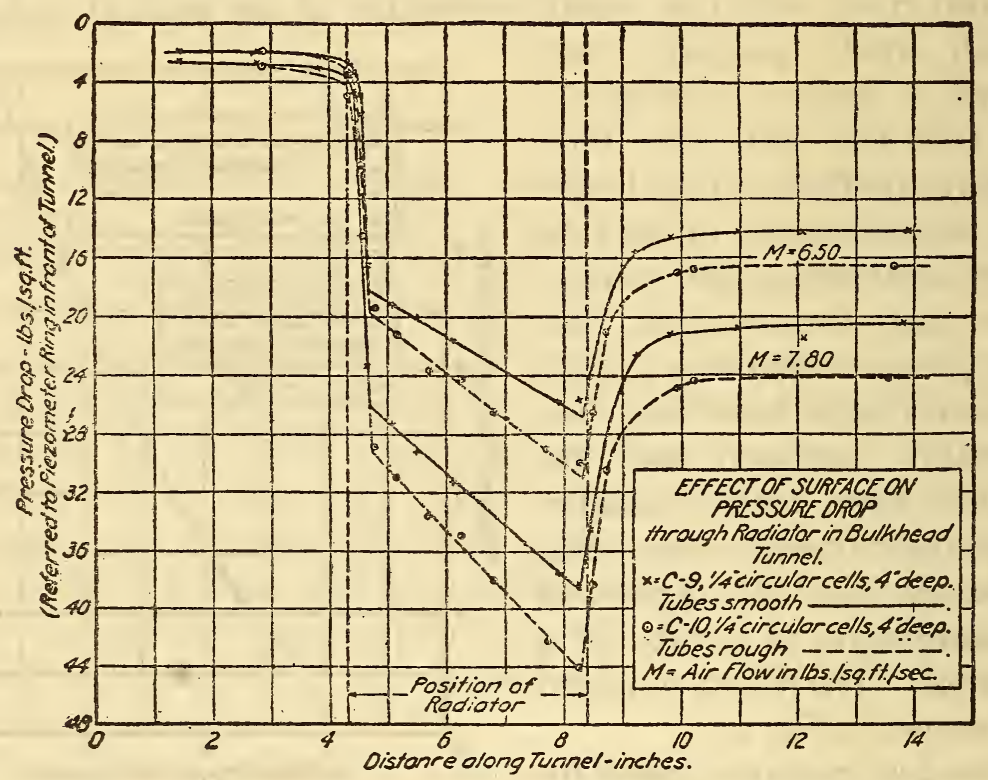

TIG. I8.-Effect of roughness of surface of the air tube of a radiator on surface friction

Two radiator cores similar in other respects show a difference of $x_{2}$ per cent in the pressure gradients for the same air flow

The statement has been made by other investigators that increase in head resistance due to temperature difference can be computed from the increase in momentum of the air as it is heated, and consequently made less dense; and it may be well to point out that while such reasoning may apply to pressure gradient for a given rate of mass flow through the radiator, there is some question about its applicability to head resistance at a given flying speed. The expansion of the air on being heated in the radiator tube tends to do two things, picturing the air stream moving and radiator stationary - to push the air out from the rear face at a higher velocity than it had at entrance, and to develop a back pressure, acting against the pressure that drives it through the tube. 
This back pressure tends to retard and reduce the air flow, and by so doing to decrease the surface friction and consequently the head resistance. On the other hand, a decrease in air flow through the radiator requires that a greater part of the approaching air shall be deflected around it, and this condition tends to increase the head resistance. Until more experimental evidence is available the question of the effect of temperature difference on head resistance must be left open, although no doubt exists as to the effect on that portion of head resistance comprised in the surface friction.

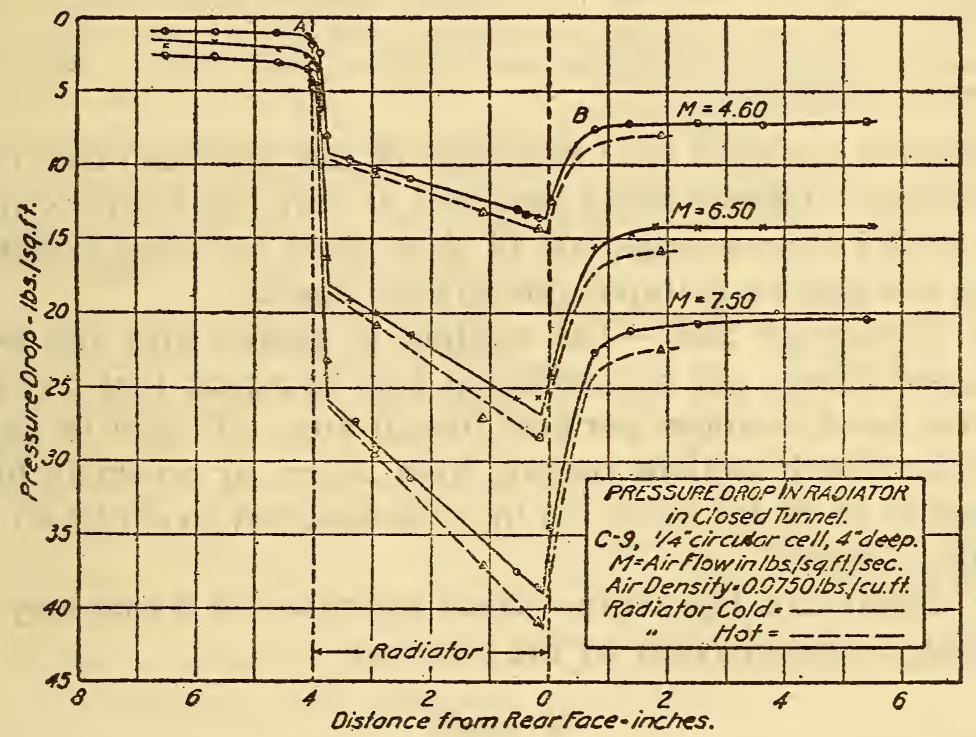

FIG. I9.-Effect of heating the metal in the walls of a radiator air tube on surface friction

For a given rate of air flow the same radiator tube was found to offer I $_{5}$ per cent more surface friction when heated $6 \mathrm{I}^{\circ} \mathrm{C}\left(110^{\circ} \mathrm{F}\right)$; the pressure gradient is $\mathrm{I}_{5}$ per cent larger

\section{HEAD RESISTANCE OF THE CORE}

35. Flying SPEEd ANd Air Density.-Head resistance of the core, including both surface friction in the tubes and "eddy resistance," is proportional to the density of the air and nearly to the square of the flying speed and over a considerable range of size is also proportional to frontal area. The relation to density is in general use by aeronautical engineers, and although an indirect verification was obtained by measurements of pressure drop made in the reduced pressure tunnel, it seems unnecessary to include a proof in this discussion. The variation with speed was determined for 30 sections of radiator core, and the powers of the speed to which the head resistances were proportional are shown in Table 8. 
TABLE 8.-Variation of Head Resistance of Core With Flying Speed

\begin{tabular}{|c|c|c|c|c|c|}
\hline Radiator No. & $\begin{array}{c}\text { Power of } \\
\text { speed }\end{array}$ & Radiator No. & $\begin{array}{c}\text { Power of } \\
\text { speed }\end{array}$ & Radiator No. & $\begin{array}{l}\text { Power of } \\
\text { speed }\end{array}$ \\
\hline$A-7 \ldots$. & 1.96 & $\mathrm{~A}-30 \ldots \ldots$ & 1.95 & $C-16 \ldots$ & 1.97 \\
\hline$A-8 \ldots \ldots \ldots . .$. & 1.94 & $A-31 \ldots \ldots \ldots \ldots$ & 1.93 & $\mathrm{D}-1 \ldots \ldots \ldots \ldots$ & 1.94 \\
\hline A-13............... & 1.96 & $A-32 \ldots . . . \ldots$. & 1.90 & $\mathrm{D}-2 \ldots \ldots \ldots \ldots$ & 1.98 \\
\hline $\mathrm{A}-15 \ldots . . . . .$. & 1.94 & B-18.......... & 2.00 & $D-3 \ldots \ldots$ & 1.98 \\
\hline$A-23 \ldots \ldots \ldots$ & 1.98 & B-19................ & 1.95 & $D-4 \ldots \ldots$ & 1.93 \\
\hline$A-25 \ldots \ldots .$. & 2.05 & B-20........ & 1.99 & $E-4 \ldots \ldots$. & 2.02 \\
\hline$A-26 \ldots . . . .$. & 1.98 & $\mathbf{B}-21 \ldots \ldots$ & 1.98 & F-6... & 2.03 \\
\hline$A-27 \ldots \ldots . .$. & 1.96 & C-2........ & 1.96 & G-1... & 1.99 \\
\hline A-28.................. & 1.94 & C-2a............. & 1.95 & G-3............. & 1.88 \\
\hline A-29................... & 1.90 & C-15............. & 1.96 & $\mathrm{G}-4 \ldots \ldots \ldots \ldots$ & 1.84 \\
\hline
\end{tabular}

Mean of above 30 values, 1.954 .

In many instances head resistance of core sections other than those listed in Table 8 was measured at only one speed, or over only a very narrow range, and in those cases the mean exponent I.954 was used for extrapolation to other speeds.

36. EFFECT OF SizE.-The relation to frontal area has been discussed above, and it is sufficient here to repeat that no variation of head resistance per unit frontal area with size or shape was found with sections ranging from $20 \mathrm{~cm}$ by $20 \mathrm{~cm}$ ( 8 by 8 inches) to $40 \mathrm{~cm}$ by $40 \mathrm{~cm}$ ( 16 by $\mathrm{I} 6$ inches) and $30 \mathrm{~cm}$ by $60 \mathrm{~cm}$ (I 2 by 24 inches).

37. EMPIRICAL EqUation.-Head resistance of a core may be computed approximately by the equation

where

$$
\mathrm{R}=c_{1} \rho S^{2}
$$

$R=$ head resistance, force per unit frontal area,

$\rho=$ air density,

$S=$ flying speed,

$c_{1}=\mathrm{a}$ constant for each type of core.

If $R$ is expressed in pounds per square foot, $\rho$ in pounds per cubic foot, and $S$ in miles per hour, $c_{1}$ ranges for cellular radiators with straight-sided air passages, from 0.013 for cores with very great free area to $0.03 \mathrm{I}$ for cores with very small free area; for fin-and-tube types, unless very open, it exceeds 0.027 ; and for irregular types with turbulence vanes it may run as high as 0.035 . If $R$ is expressed in $\mathrm{kg} / \mathrm{m}^{2}, \rho$ in $\mathrm{kg} / \mathrm{m}^{3}$, and $S$ in $\mathrm{m}$ per sec, the corresponding values of $c_{1}$ are, respectively, $0.020,0.047,0.04 \mathrm{I}$, and 0.054 . 
38. EFFECTS OF DESIGN OF CORE.-The factor $c_{1}$ is a function of the form and dimensions of the core and of the condition of the cooling surfaces, but the form of the function has not been determined. The features of design that cause air flow to be low are almost, if not quite, identical with those which cause high head resistance. Head resistance increases with decrease of free area and with increase of depth, although the increase with depth is not rapid. The eddy resistances at the front and rear faces are so great that if the sides of the air passages are straight and fairly smooth an increase of an inch or two in their length does not increase the total resistance very greatly. The effect of smoothness of tube surface on head resistance is evidently in the same direction as the effect on surface friction in the tubes, but less pronounced because the surface friction constitutes only a part of the resistance. Polishing the surfaces may reduce the resistance slightly, but no very marked effect will be produced without a considerable change in the character of the surface. Head resistance of a core of the type $\mathrm{C}-8$, with headers (water boxes, top and bottom), was measured both before and after the tubes had been smoothed. The differ-

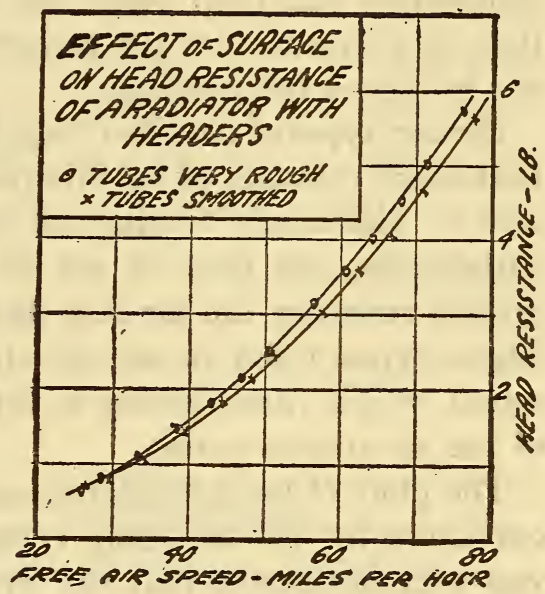

FIG. 20.-Effect of roughness of surface of air tubes of a radiator on head resistance of the radiator

With unusual extremes of roughness a difference of 7 per cent of the resistance of the radiator, or to per cent of the resistance of the core, was found ence in resistance is shown in Fig. 20. The core of the section used was $20 \mathrm{~cm}$ ( 8 inches) square, and at $27 \mathrm{~m}$ per sec (6o miles per hour) the difference in resistance is seen by the plot to be about 0.24 pound. The resistance of an 8-inch section without headers is, from Table 2 I, 2.53 pounds at this speed, or the change in resistance due to smoothing the tubes was nearly ro per cent. This difference is greater than would ordinarily be obtained by polishing the tubes of a radiator because the surface was unusually rough. Head resistance is increased considerably by projections, indentations, holes, or discontinuities of any kind in the walls of the air passages, since they introduce eddy resistance into the passages themselves. The types B-I 9 and B-20 illustrate the effect of holes in the tube $75013^{\circ}-22-4$ 
walls, the head resistance constant of the perforated type B-I9 being 13 per cent greater than that of the unperforated type, although the latter is one-half inch deeper. Except in perforations and in depth the types are similar, the dimensions and form matching B-I (Fig. 7I). Fin-and-tube types show particularly high head resistance, often partly because of their small free area, but even in the more open types the eddy resistances set up at each water tube make the total resistance very high. Spiral vanes, or other devices for increasing turbulence of the air, increase the head resistance greatly, and in every case where a reliable comparison has been made the increase has been found greater than is warranted by the increase in heat dissipation except for use in obstructed positions.

Certain types of core $\mathrm{E}-\mathrm{I}$ to $5, \mathrm{E}-9$, and $\mathrm{E}$-1o are so constructed that short columns of air vibrate across the direction of air flow, and by alternately forcing air into the fast moving stream and withdrawing air from it act as a very effective drag upon it, greatly reducing the air flow and increasing the head resistance. These types when in an air stream produce a shrill whistling sound, which varies widely in intensity and in pitch as the speed of the air stream varies.

The plots of head resistance against flying speed show irregular curvature for the whistling types, and in taking observations it was very noticeable that the irregularities occurred at speeds at which the tone of the whistle changed suddenly.

39. $\mathrm{YAW}_{\mathrm{AW}}$ - - The effect of yawing the radiator, or turning it so that the air strikes it at some other angle than normal to its face, is, in general, to increase the resistance for angles of yaw (angle between direction of the stream and the normal to the face) up to at least 30 or $40^{\circ}$. Fig. 2 I shows typical curves of resistance in terms of angle of yaw. The types $\mathrm{A}-\mathrm{I} 3$ and $\mathrm{C}-\mathrm{I} 5$ are representative of ordinary cellular radiators, while the others are unusual types of core.

\section{HEAD RESISTANCE CHARGEABLE TO THE RADIATOR}

Head resistance chargeable to the radiator includes surface friction, eddy resistance at the radiator, and every effect of the radiator and aircraft upon each other that makes the total resistance of the craft greater than it would need to be if it could be designed without a radiator.

40. Unobstructed Positions.-For unobstructed positions the head resistance of the core may be regarded as equal to the head 

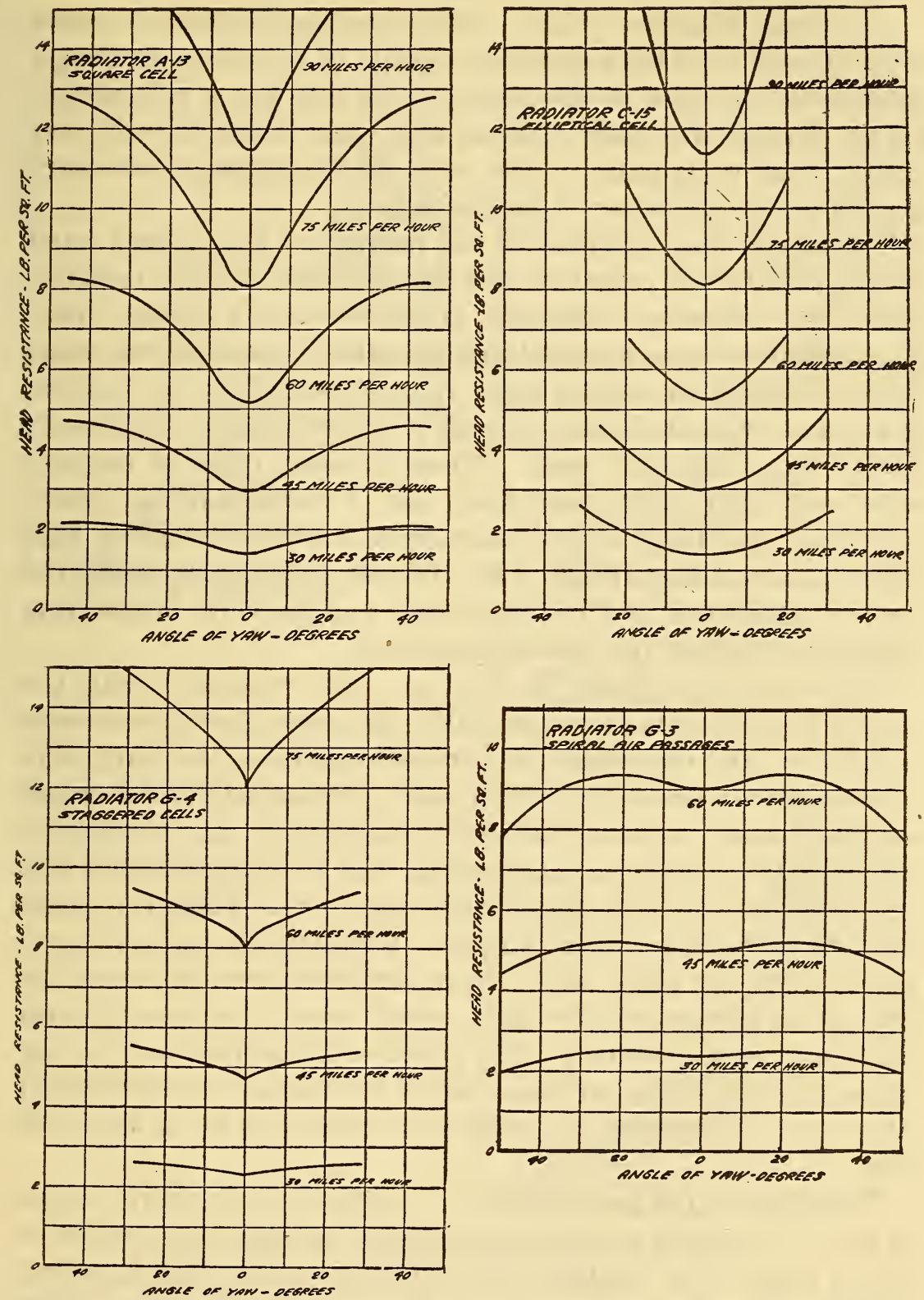

FIG. 2I.-Effect of yaw on head resistance of a section of radiator core Upper charts are for cells of common types, lower ones for unusual types of core 
resistance chargeable to the radiator, except for the comparatively small resistance of the tanks or headers and connecting pipes.

4I. Nose Position.-The model fuselage described above (art. 23) was used for a qualitative study of the effect of using a radiator in the nose of the fuselage. It was not a true model, for no attempt was made to build it to scale, but it showed very clearly that a radiator in the nose of the fuselage adds very greatly to the resistance of the fuselage.

The model was mounted on the balance of the 54 -inch wind tunnel, and its head resistance was measured like that of a radiator, under the following conditions: (I) Nose crudely stream lined; (2) stream-line nose removed and a radiator placed in the nose, but covered with a sheet of paper to exclude air flow; (3) radiator in the nose; with air flowing through it, and with side vents closed, partly open, and wide open. Three different types of radiator were used-B-I, very open; $A-23$, one of the best of the square cell types; and $\mathrm{G}-3$, a very compact type characterized by high heat transfer at low air flow. No attempt was made to design the best possible nose, and the form used was made by intersecting cylindrical surfaces but served its purpose.

The results are shown in Fig. 22. The resistance with the stream-line nose was about one-half of that with the flat nose with no air flow, and the resistance increased rapidly as the vents were opened and air allowed to flow through. Reasons for this increase are easily seen. The air that enters the radiator does not have a clear, straight path out of the fuselage, but is greatly retarded and must find its way out through the sides, ${ }^{18}$ after which it is again brought up to speed by the stream. Furthermore, any air escaping through the sides of the body interferes seriously with the flow of the stream past the body, which might otherwise conform fairly well to stream lines. The addition of louvres over the side openings to direct the air backward as it came out into the stream was found to decrease the resistance somewhat for a given air flow.

The effect of the nose radiator is, perhaps, most clearly shown in Fig. 23, in which a comparison is made between the installation in the nose of the fuselage and a radiator of the same type and equivalent cooling capacity in an unobstructed position. The curves are computed for each of the three radiators used, and for flying speeds of 60 and 90 miles per hour ( 27 and $40 \mathrm{~m}$ per second),

18 This statement applies to the wind tunnel. In actual flight the air is picked up by the fuselage as by a scoop and acquires a speed approaching that of the plane before making its escape. 

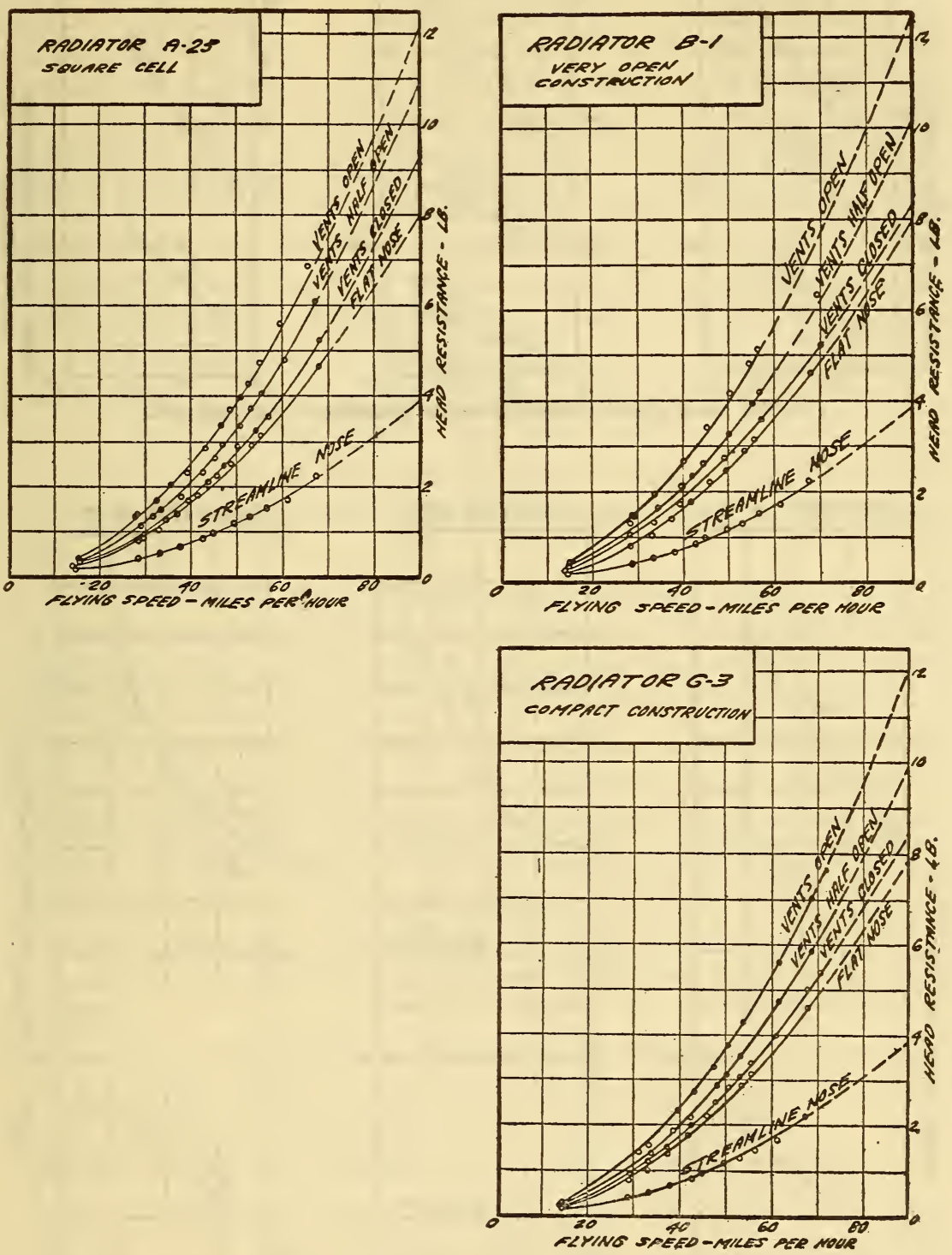

FIG. 22.- Head resistance of model fuselage with nose radiators

The differences in total head resistance are not large, notwithstanding great differences in cell structure and in heat transfer properties of the several radiator cores illustrated. Note great increase in total head resistance of fuselage with any nose radiator over the value with a stream-line nose 

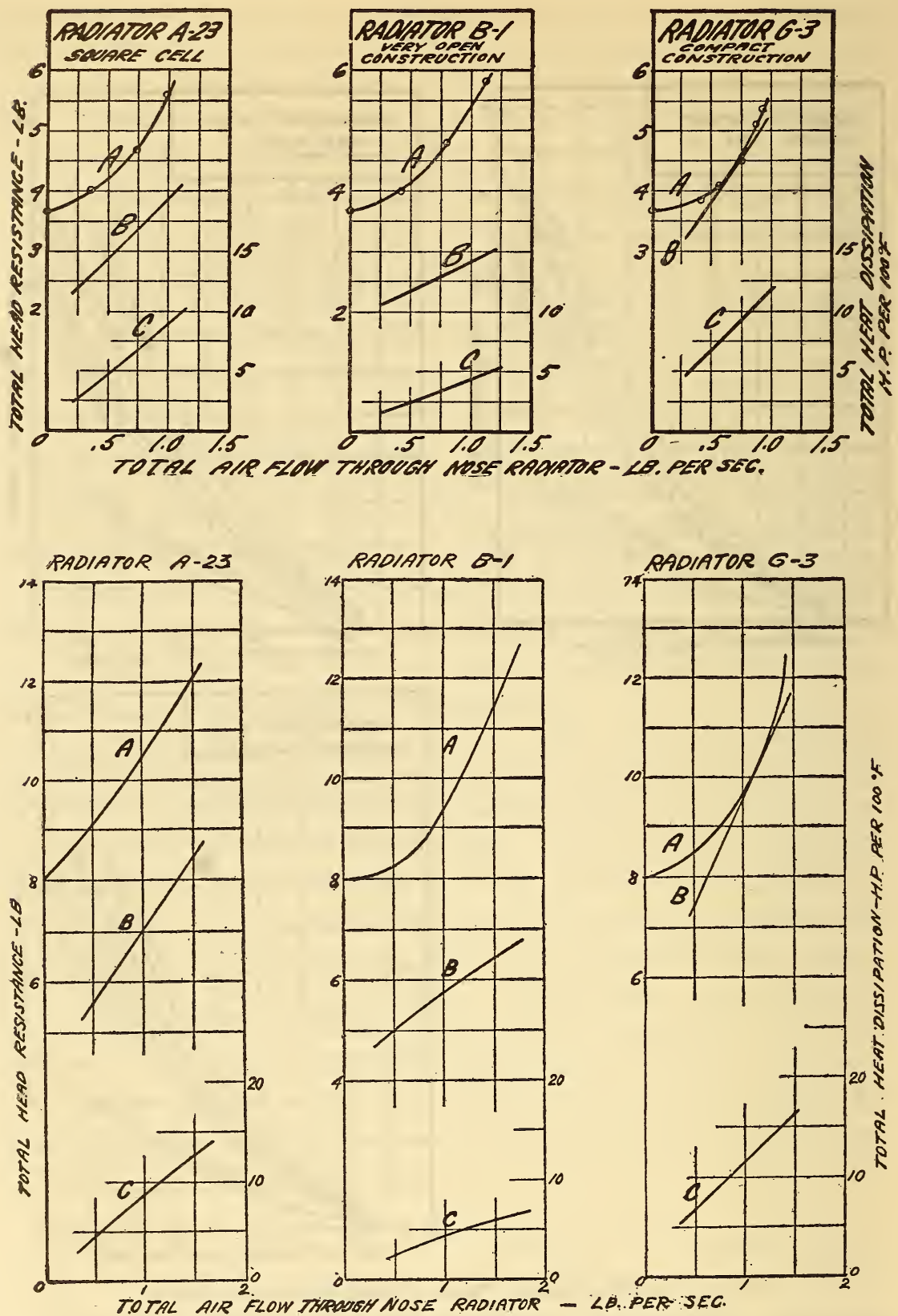

Fig. 23.-Comparative value of the head resistance offered by a fuselage with radiator mounted in nose, and same fuselage, stream lined, plus a radiator of equal cooling effectiveness and similar construction, mounted in unobstructed position

The latter arrangement is shown to be decidedly superior for the three types of core tested at the wind speeds of 60 and 90 miles per hour (26.8 and 40.2 meters per second). Symbols A, B, C, explained on p. 299. 
showing resistances in terms of the amount of air flowing through the nose radiator. Curve $A$ is in each case the resistance of the model fuselage with its nose radiator, while curve $B$ shows on the same scale the sum of the resistance of the fuselage when fitted with the stream-line nose and the resistance of a section of the same kind of core in an unobstructed position of such size as to give the same rate of heat dissipation. Lest the curves might seem to indicate that the three types of radiator are about equally good in the nose position, but greatly different in unobstructed positions, a third curve $C$ is added, showing heat dissipation for each type in terms of air flow through the core, and a comparison of heat dissipation shows that a much larger radiator of the type B-I would be needed to cool a given engine than of the type G-3. In fact, if such a type as B-I were used in the nose of the fuselage to cool an engine of fairly high power, so large a radiator would probably be required as to necessitate enlargement of the fuselage to contain it.

The curves should not be interpreted to mean that the type G-3 is good for the nose position, but rather that if a radiator is to be taken from an unobstructed position and placed in the nose of the fuselage this type would show less ill effect than the others. It has very high head resistance in unobstructed positions. If, in spite of the great increase in head resistance of the assembly, the designer insists upon mounting the radiator in a nose position he should choose a type that will give maximum heat transfer with very low air flow, even at the expense of high head resistance of the core, since any considerable air flow through the core will increase to a marked degree the head resistance chargeable to the radiator.

42. Wing Positions. - A limited study of the effect of a radiator on the properties of a wing was made by the use of the half-size section of wing described above (art. 24). Both the "lift" and "drift" of the wing section were measured before the fabric had been cut away for the radiator and after the radiator had been inserted. The measurements were made in the 54-inch wind tunnel on the balance described above (art. 27), which was suspended on an arm of a second balance, so that vertical forces ("lift") could be measured as well as "drift." With the object of determining approximately the center of pressure on the wing, the balance arm supporting it was extended to two different positions. The computations involved taking the differences between quan- 
tities so nearly equal that the differences were but little greater than the limits of experimental error. The results were naturally very erratic, requiring considerable fairing to give smooth curves, and must be regarded as only qualitative, since errors of 8 per cent in lift and 25 per cent in drift are quite probable. Four angles of incidence were used, and Fig. 24 shows that the radiator may cause a decrease in drift at certain positions and an increase at others, while it decreases the lift at all angles.

43. Slip-Stream Positions.-If the radiator is in the propeller slip stream, the head resistance chargeable to it is probably approximately equal to what it would be if the propeller could be removed and the craft driven by other means at such a speed as would give the same speed of air past the radiator. If this is true, an assumption that the slip-stream speed is, for example, 20 per cent greater than the speed of flight would mean an increase of 44 per cent in head resistance over the value corresponding to the flying speed, since the resistance varies as the square of the speed. A slight further increase is caused by the swirl of the slip stream, which is similar to the effect of yawing the radiator. On the other hand, the effect of slip stream for positions such as the nose of the fuselage, which would be obstructed without the propeller, is that the virtual yaw of the slip stream tends to decrease the air flow through the core and may to a small extent compensate for the increased slip-stream speed by reducing the head resistance chargeable to the radiator, since for such positions high air flow is accompanied by high head resistance.

\section{E. POWER ABSORBED}

As stated above, the power absorbed by a radiator may be divided into two parts, due to head resistance and to weight; and, as in the case of head resistance, the power absorbed by the core is only a part of that chargeable to the radiator. ${ }^{19}$

44. Power Absorbeid by THE CORE. - The power absorbed in overcoming the head resistance of the core is equal to the product of the head resistance by the flying speed and by a conversion factor depending upon the units used, and since the head resistance is approximately proportional to the square of the speed this fraction of the power absorbed is approximately proportional to the cube of the speed. The weight is supported by a vertical component of the air pressure on the wing, called the "lift,"

\footnotetext{
${ }^{19}$ Since in this report power absorbed is reduced to unit frontal area, the statements apply to power
} absorbed by a particular radiator rather than to the power absorbed in cooling a given engine. 

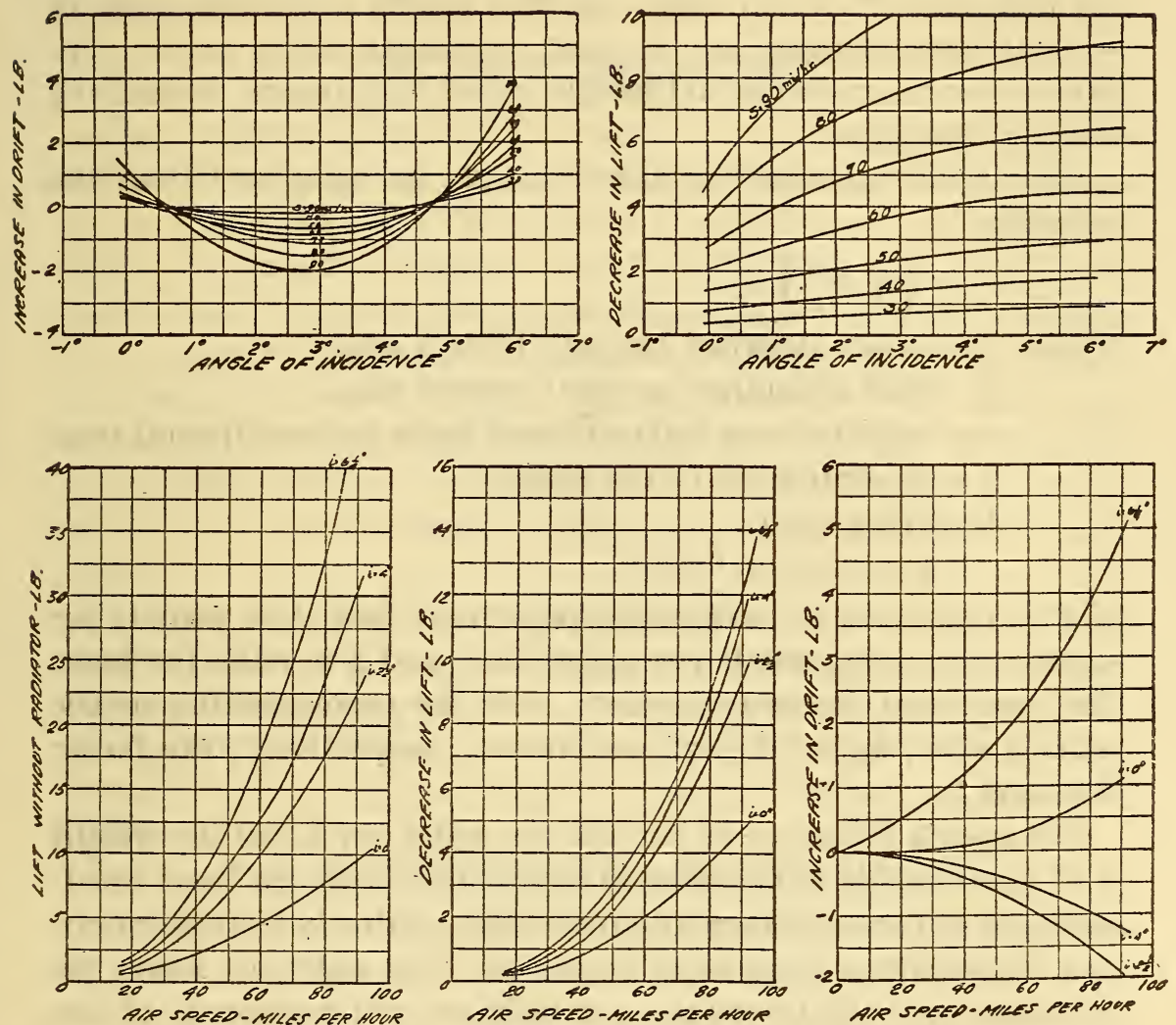

Fig. 24.-Effect of a radiator in wing section upon the aerodynamic forces acting on the wing

Upper curves show that for all speeds there is a decrease in lift, regardless of angle of incidence, $i$, but that drift may be either increased or decreased by the presence of the radiator, depending on angle of incidence. Lower curves show same data plotted with air speed, $s$, as abscissas. (A radiator is a partial "hole" in the wing, permitting restricted passage of air, thus breaking down the pressure difference which it is the function of the wing to preserve, and from which the lifting force on the plane is obtained) 
which is accompanied by a corresponding horizontal component, or "drift." The ratio of "lift" to "drift" varies for different types of wing and for different angles of attack, but if it is known for the particular craft on which a radiator is mounted the weight may be reduced to an equivalent head resistance by dividing by this ratio of lift to drift. In this report a lift-drift ratio of 5.4 has been assumed as one that represents rather severe conditions for the radiator and assigns equal importance to climbing and to level flight.

The power absorbed by the core may be computed from the equation

$$
P=c\left(R+\frac{w}{r}\right) S
$$

where $P=$ power absorbed per unit frontal area,

$R=$ head resistance per unit frontal area,

$w=$ weight of core and contained water per unit frontal area,

$r=$ lift-drift ratio of the plane,

$S=$ flying speed,

$c=$ a conversion factor.

If $P$ is expressed in horsepower per square foot, $R$ in pounds per square foot, $w$ in pounds per square foot, and $S$ in miles per hour, the conversion factor is 0.00267 . For the corresponding metric units $\mathrm{kw} / \mathrm{m}^{2}, \mathrm{~kg} / \mathrm{m}^{2}, \mathrm{~kg} / \mathrm{m}^{2}$, and $\mathrm{m} / \mathrm{sec}$., respectively, the factor is 0.0098 .

For speeds below $30 \mathrm{~m}$ per sec. (60 miles per hour) the weight is of considerable importance in comparison with the head resistance, but as the speed increases it becomes relatively less important, and for speeds as high as $45 \mathrm{~m}$ per sec. (roo miles per hour) the difference in weight between one radiator and another is of but small importance.

45. Power Absorbed Chargeable to the Radiator.-Equation (I) (art. 44), for power absorbed by the core, is also applicable to power absorbed chargeable to the radiator, if head resistance chargeable to the radiator is substituted for head resistance of the core, but the equation is of limited use because of the difficulty of obtaining the value of head resistance chargeable to the radiator. This equation ignores the power used in driving the water pump, which is usually a very small part of the engine power, and has been omitted from this discussion.

Power absorbed chargeable to the radiator is evidently high for such installations as cause high head resistance chargeable to the radiator, notably the position in the nose of the fuselage 
and slip-stream positions. Since the part of the power absorbed that is due to head resistance alone (neglecting weight) is proportional to the cube of the speed, an assumption of 20 per cent increased air speed in the slip-stream represents an increase of 73 per cent in that part of the power absorbed, while the increase in the part due to weight is only 20 per cent.

\section{HEAT DISSIPATION}

The transfer of heat from water to air takes place in three stages-from water to metal, through the metal, and from metal to air. In each stage the transfer is proportional to the appropriate temperature difference, and in the first and third it depends upon the speed and turbulence of flow. The heat transfer is not proportional to the amount of cooling surface provided in the radiator for two reasons: The air is warmed and the water cooled appreciably in passing through the radiator, so that the temperature difference between them is less at the rear face than at the front, and indirect cooling surface is less effective than direct.

It seems reasonable to assume that the transfer of heat from water through the metal to the air is proportional to the difference in temperature between the water and the air, and as an experimental verification of the assumption was found in a French report ${ }^{20}$ it was adopted without further experimental study.

\section{A. EXPERIMENTAL METHOD AND COMPUTATION}

46. WIND TUNNELS.-The "steam" tunnel partially described in article 7 (Figs. 2, 3) as first built was equipped for using superheated steam as a source of heat, hence its name. As rebuilt later, hot water was used for calorimetric measurements in place of steam, and parts of the equipment were borrowed from the "reduced pressure" wind tunnel. The wind tunnel equipment will accordingly be described under three subtitles-steam tunnel using steam, reduced pressure tunnel (using water), and steam tunnel using water.

(a) STEAM TUNNEL USING STEAM

47. Apparatus.-In the steam tunnel as first built air was pushed through the channel by a 36 -inch (9I cm) Sturtevant blower driven by a 220-volt direct-current shunt motor rated at Io h. p. $(7.5 \mathrm{kw})$. By the use of rheostats in both field and arma-

${ }^{2}$ Bulletin de la Section Technique de l'Aeronautique Militaire, August, I9I8, p. $2 \%$ 
ture circuits and by throttling the intake of the fan air speeds from 4.5 to $45 \mathrm{~m}$ per sec. (Io to roo miles per hour) could be obtained in the working section, which was square, $20.3 \mathrm{~cm}$ (8 inches) inside measurement. The tunnel was built in two sections to allow insertion of the radiator, the outlet section being supported by a frame on casters. The parts adjacent to the radiator were inlaid with sheet brass and equipped with pitot grid and piezometer rings connected to pressure gages, as described above (art. 7), and indicated in Fig. 3. Extra boards on the sides and bottom of the tunnel facilitated alignment of the two sections and the radiator.

The steam connections are shown in Fig. 25. Steam from the high-pressure mains was passed through a controlling valve, where its pressure was reduced to about $0.7 \mathrm{~kg} / \mathrm{cm}^{2}$ (Io pounds per square inch); a $U$-shaped pipe at the bottom of which a valve partly open allowed most of the water to drain out; a coil, where it was heated to from Iro to $150^{\circ} \mathrm{C}\left(230\right.$ to $\left.300^{\circ} \mathrm{F}\right)$; a plug cock, where its pressure fell to approximately atmospheric; a separator, where its temperature was measured with a mercury thermometer; the radiator, where most of it was condensed; and another separator from which the condensed water dropped into a weighing tank.

In preliminary work a thermometer was inserted in the lower header of the radiator, but the water temperature was found to vary so slightly from the temperature of condensation that the heat involved in the cooling of the water in the radiator could be neglected. Magnesia pipe covering was used to reduce heat losses from the steam pipes, and the sides of the radiator were lagged with cork board $1.3 \mathrm{~cm}$ ( $1 / 2$ inch) thick. The headers were sometimes covered with hair felt, but their surface was so small and the air flow past them so low that errors due to loss of heat from the headers were neglected.

48. OBSERVATIONS.- The drain valves in the steam line were opened, the water drained off, and the blower for the air stream in the wind tunnel started. When the water was out of the line and the steam flowing well, the gas heater was lighted, the blower adjusted to give the desired speed, and the valves and the heater adjusted so that steam showed faintly at the lower separator. After steady conditions of temperature and steam flow had been attained one observer set the balance slightly ahead of the actual weight and started a stop watch when the pointer of the balance crossed the zero line. Then weights of from I to ro pounds, depending upon the probable rate of condensation, were added to 
the balance. One observer recorded steam temperatures and adjusted the valves when necessary to maintain steady flow, while the other observer read the differential pressure gages and the temperature of the air at entrance to the radiator. About a minute before the assigned weight of steam was condensed a bell warned the observers; who prepared to note the time at which the pointer of the balance again crossed the zero line. The time

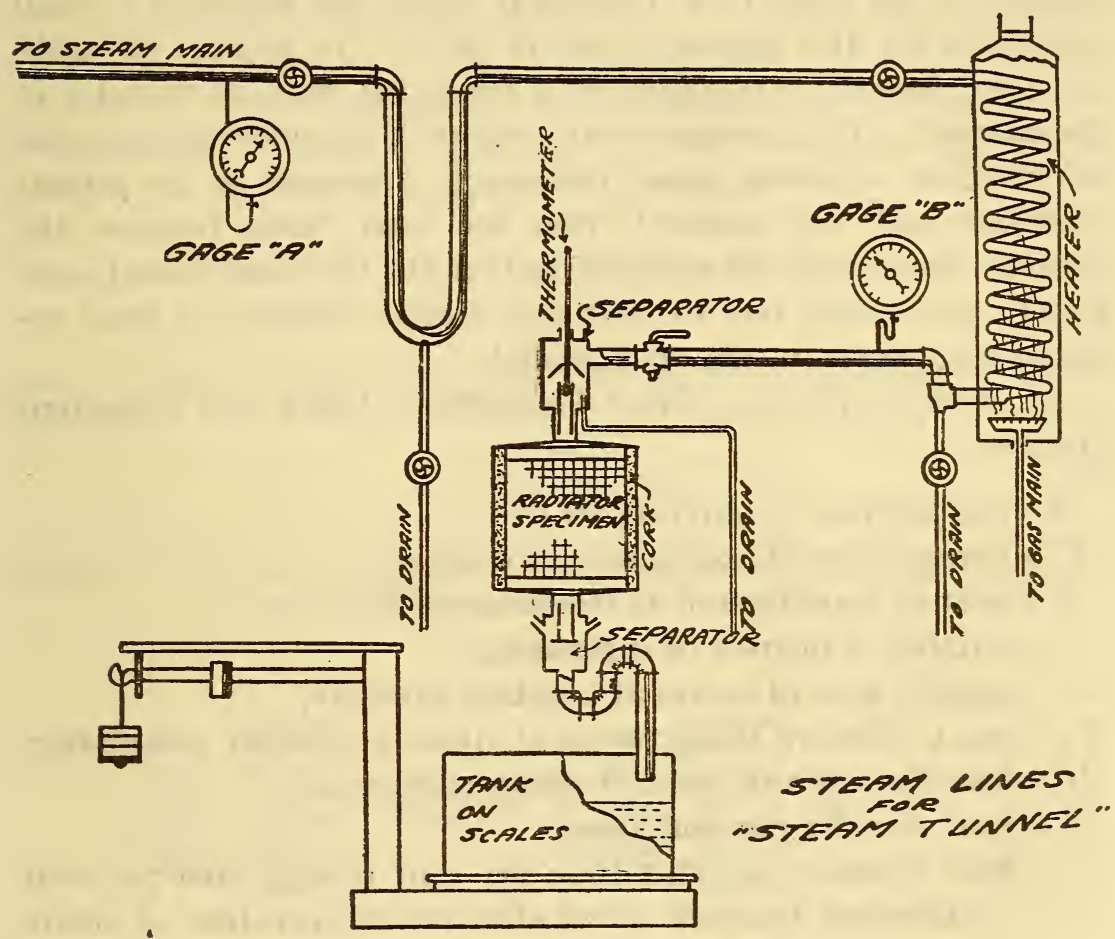

FIG. 25.-Diagram of steam connections for calorimetric measurement of radiators in small wind tunnel

The heat delivered to the air stream per unit time was measured by weighing steam condensed in the radiator water tubes

interval varied between three and six minutes and could easily be measured to one second. The balance readings were within o.or pound ( $5 \mathrm{~g})$. At each air speed two or three runs were made for a check.

The observed quantities included (I) differential pressure between the pitot grid and its piezometer ring, (2) differential pressure between piezometer rings before and behind the radiator, (3) temperature of the air in front of the radiator (read on a mercury thermometer), (4) temperature of steam at entrance to radi- 
ator, (5) weight of steam condensed, (6) time of condensation, (7) barometric pressure in the room, (8) relative humidity, (9) differential pressure between the channel in front of the radiator and outside air.

49. Computation of Arr Flow.-Air flow was computed as explained in article ro. Pressure drop in the air stream in passing through the radiator was computed by multiplying the reading of the gage by a conversion factor and applying a small correction for the pressure drop in $36 \mathrm{~cm}$ ( 14 inches) of unobstructed channel, determined by a blank run with no radiator in the channel. This measurement, originally intended to give some information regarding head resistance, appeared to be almost valueless and was omitted from the later tests, because the pressure drop when the radiator section fills the wind tunnel completely was found not to bear any simple relation to head resistance measured in the open tunnel.

5o. Computation of Heat Transfer.-Using any consistent units, let

$\theta=$ temperature of entering air,

$T_{\mathrm{c}}=$ temperature of condensation of steam,

$L=$ heat of vaporization at temperature $T_{\mathrm{c}}$,

$s=$ number of degrees of superheat,

$C_{\mathrm{p}}=$ specific heat of steam at constant pressure,

$T_{\mathrm{m}}=$ mean effective temperature of steam and water in radiator,

$H_{\mathrm{s}}=$ heat lost by unit mass of steam condensed,

$H_{1}=$ heat transfer per unit time,

$H_{2}=$ heat transfer per unit time per unit frontal area per unit difference between mean effective temperature of steam and water and temperature of entering air,

$w=$ weight of steam condensed,

$\tau=$ time of condensation,

$c, c^{\prime}=$ conversion factors, depending upon units used.

Now

$$
H_{\mathrm{s}}=L+C_{\mathrm{p}} s .
$$

In computing the mean effective temperature of the steam and water the temperatures were weighted according to the amount of heat lost at each temperature, so that $T_{\mathrm{m}}$ was defined as

$$
T_{\mathrm{m}}=\frac{\left(T_{\mathrm{c}}+\frac{s}{2}\right) C_{\mathrm{p}} s+T_{\mathrm{c}} L}{C_{\mathrm{p}} s+L} .
$$


With the aid of equation (I) this may be reduced to

$$
T_{\mathrm{m}}=T_{\mathrm{c}}+\frac{C_{\mathrm{p}}}{2 H_{\mathrm{s}}} s^{2}
$$

The term $\frac{C_{\mathrm{p}}}{2 H_{\mathrm{s}}}$ represents a small correction and is a function of the superheat $s$, since the range of condensation temperatures is so small that the heat of vaporization may be regarded as constant. For convenience in computation Table 9 of values of this correction for different values of $s$ was computed from steam-table data. Similarly, the heat lost $H_{\mathrm{s}}$ was tabulated from different amounts of superheat. The rate of transfer of heat from the steam or water to the air, in units of power, is

$$
H_{1}=c \frac{H_{\mathrm{s}} w}{\tau}
$$

and the rate of transfer per unit time per unit temperature differ ence is

$$
H_{2}=c^{\prime} \frac{H_{\mathrm{s}} w}{\tau\left(T_{\mathrm{m}}-\theta\right)} \cdot
$$

TABLE 9.-Properties of Superheated Steam Required for Computing Results of

\begin{tabular}{|c|c|c|c|c|c|c|c|}
\hline \multirow{3}{*}{$\begin{array}{c}\text { Super- } \\
\text { heat } \\
\text { above } \\
\text { tempera- } \\
\text { ture of } \\
\text { conden- } \\
\text { sation } \\
\\
{ }^{\circ} \mathrm{C} \\
5\end{array}$} & \multirow{3}{*}{$\begin{array}{c}\text { Effective } \\
\text { tempera- } \\
\text { ture cor- } \\
\text { rection } \\
\text { above } \\
\text { tempera- } \\
\text { ture of } \\
\text { conden- } \\
\text { sation } \\
\\
{ }^{\circ} \mathrm{C} \\
0.01\end{array}$} & \multicolumn{2}{|c|}{$\begin{array}{l}\text { Heat per unit } \\
\text { mass of steam } \\
\text { condensed }\end{array}$} & \multirow{3}{*}{$\begin{array}{c}\begin{array}{c}\text { Super- } \\
\text { heat } \\
\text { above } \\
\text { tempera- } \\
\text { ture of } \\
\text { conden- } \\
\text { sation }\end{array} \\
{ }^{\circ} \mathbf{F}\end{array}$} & \multirow{3}{*}{$\begin{array}{c}\text { Effective } \\
\text { tempera- } \\
\text { ture cor- } \\
\text { rection } \\
\text { above } \\
\text { tempera- } \\
\text { ture of } \\
\text { conden- } \\
\text { sation } \\
\\
\\
\text { oF } \\
0.02\end{array}$} & \multicolumn{2}{|c|}{$\begin{array}{l}\text { Heat per unit } \\
\text { mass of steam } \\
\text { condensed }\end{array}$} \\
\hline & & & hp. sec. & & & & hp. sec. \\
\hline & & $\begin{array}{r}\mathrm{g} \mathrm{cal} / \mathrm{g} \\
541.2\end{array}$ & $\begin{array}{l}\text { lb. } \\
1377\end{array}$ & & & $\begin{array}{c}\text { Btu/lb. } \\
974.7\end{array}$ & $\begin{array}{l}\text { Ib. } \\
1378\end{array}$ \\
\hline 10 & .04 & 543.6 & 1383 & 20 & .10 & 979.4 & 1385 \\
\hline 15 & .10 & 545.9 & 1389 & 30 & .22 & 984.1 & 1391 \\
\hline 20 & .17 & 584.3 & 1395 & 40 & . 38 & 988.8 & 1398 \\
\hline 25 & .27 & 550.6 & 1401 & 50 & .59 & 993.5 & 1405 \\
\hline 30 & .38 & 553.0 & 1407 & 60 & .85 & 998.2 & 1411 \\
\hline 35 & .52 & 555.3 & 1413 & 70 & 1.15 & 1002.9 & 1418 \\
\hline 40 & .67 & 557.7 & 1419 & 80 & 1.49 & 1007.6 & 1424 \\
\hline 45 & .85 & 560.0 & 1425 & 90 & 1.88 & 1012.2 & 1431 \\
\hline 50 & 1.05 & 562.4 & 1431 & 100 & 2. 31 & 1016.9 & 1438 \\
\hline 60 & 1.49 & 567.1 & 1443 & 125 & 3.56 & 1028.5 & 1454 \\
\hline 70 & 2.02 & 571.8 & 1455 & 150 & 5.07 & 1040.3 & 1471 \\
\hline 80 & 2.61 & 576.5 & 1467 & 175 & 6.81 & 1051.9 & 1487 \\
\hline & & & & 200 & 8.82 & 1063. 7 & 1504 \\
\hline
\end{tabular}
Radiator Tests Using Steam as a Source of Heat

[Pressure $=15$ lbs./in. $\left.{ }^{2}\right]$ 
The temperature difference $\left(T_{\mathrm{m}}-\theta\right)$ was chosen instead of a difference between mean steam and water temperature and mean air temperature merely for convenience. The justification of this choice is given in Appendix E.

(b) REDUCED PRESSURE TUNNEL

51. Apparatus-Air System.-The construction of the reduced pressure wind tunnel is shown diagrammatically in Fig. 6. Both the tunnel and the fan used for drawing air through it were inclosed in a steel tank about $1.5 \mathrm{~m}$ ( 5 feet) long and $90 \mathrm{~cm}$ (3 feet) in diameter, sufficiently tight to allow maintaining pressures as low as 30 to $40 \mathrm{~cm}$ ( 12 to 16 inches) of mercury. The fan was driven by a direct-current shunt motor rated at $5.5 \mathrm{kw}$ ( 7.5 horsepower), which could be run on either I Io or 220 volts, and for further speed control was provided with rheostats in both field and armature circuits. A $38 \mathrm{~cm}$ ( 15 inch) Sirocco fan was used.

At the entrance to the tunnel a honeycomb of sheet-metal cells $2.5 \mathrm{~cm}$ ( $\mathrm{I}$ inch) square and $7.5 \mathrm{~cm}$ (3 inches) deep served to break up vortices and straighten the air flow. Following this in order came: (I) Pitot grid and piezometer ring for measuring air speed, (2) electric resistance thermometer for measuring air temperature, (3) thermocouple grid containing one set of junctions for measuring the rise in temperature of the air in passing through the radiator, (4) piezometer ring for measuring the drop in pressure in the air stream in passing through the radiator, (5) the radiator section, (6) third piezometer ring, (7) thermocouple grid containing the second set of junctions for measuring rise in air temperature, (8) fan, (9) conical guide vanes to deflect the air around the end of the tunnel and back around the outside of it, (Io) wet-and-dry bulb hygrometer (in the return air stream), (I ) cold-water radiator for cooling to original temperature the heated air during its return to the entrance of the tunnel.

The electric resistance thermometer for the measurement of air temperature at entrance to the radiator is described above (art. II) and shown in Fig. 7. The thermocouple grids for the calorimetric measurement of rise in air temperature contained 25 copper constantan couples, with junctions equally spaced over the channel. An attempt was made to measure the rise in air temperature by the use of $200-0 h m$ resistance thermometers of fine insulated nickel wire strung over a frame of iron wire, but the varying strains in the fine wire caused by the air stream resulted in such changes in resistance as to introduce large errors. 
The thermocouples were calibrated, and the calibration expressed in the form

or

$$
E=a \theta+b \theta^{2}
$$

$$
\frac{d E}{d \theta}=a+2 b \theta
$$

where $E=$ electromotive force of the couples, with cold junction at $\mathrm{o}^{\circ} \mathrm{C}$.

$$
\begin{aligned}
\theta & =\text { temperature in degrees centigrade. } \\
a, b, & =\text { constants. }
\end{aligned}
$$

This form of calibration was used throughout the work on radiators, the constants being determined for each spool of wire used.

52. Apparatus-Water System.-The source of heat for calorimetric measurements in the reduced pressure wind tunnel was hot

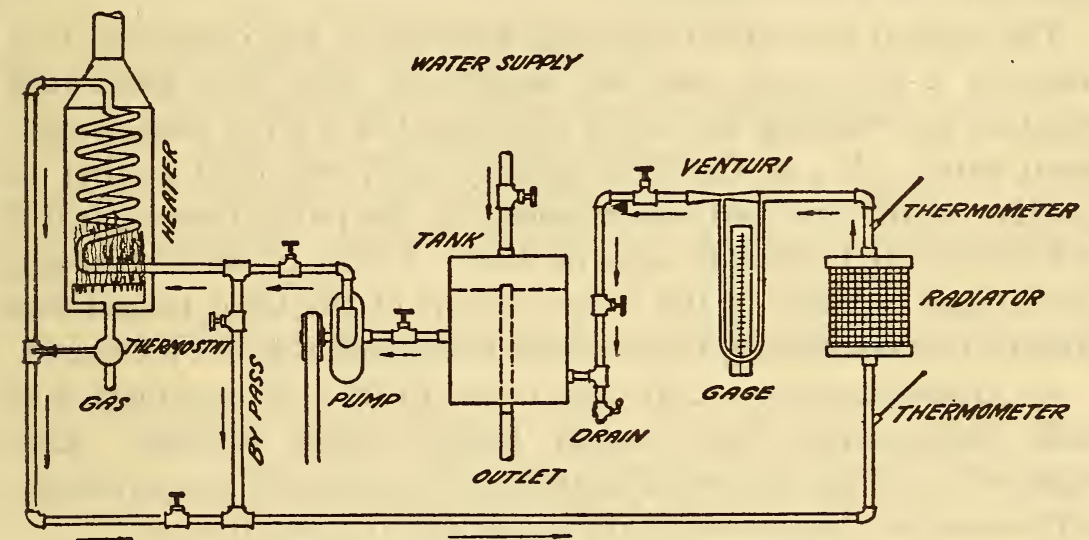

FIG. 26.-Diagram of water system for calorimetric measurements of radiators in both the reduced pressure wind tunnel and the "steam tunnel" as rebuilt, superseding steam by hot water as the source of heat

water. The flow of water was produced by a centrifugal pump driven by a direct-current shunt motor running on 220 volts and rated at $750 \mathrm{w}$ (I horsepower). A diagram of the piping system is shown in Fig. 26. The resistance of the coil of the gas heater was large in comparison with that of the remainder of the line, and a by-pass was provided for obtaining the higher rates of flow. A thermostat in the heater served to maintain approximately constant water temperature at the entrance to the radiator. After the heater, there followed in order: (I) Mercury thermometer in the water line used when necessary to adjust the thermostat, (2) one set of thermoelectric junctions for measuring drop in temperature of the water in passing through the radiator, (3) radiator 
section, (4) second set of junctions of the thermocouples, (5) venturi meter for measuring the rate of water flow, (6) surge tank.

For the measurement of the drop in temperature of the water 2 I thermocouples, with the similar junctions about $\mathrm{I} .3 \mathrm{~cm}(\mathrm{I} / 2$ inch) apart, incased in a copper tube in the form of a spiral coil, were placed in the pipes at inlet and outlet of the radiator. Previous work showed that thermocouples in the center of the water boxes did not give a satisfactory average temperature. In addition to the use of these thermocouples differentially to measure accurately the fall of temperature of the water, a connection to a reference junction in an oil bath whose temperature was measured to o. $\mathrm{I}^{\circ} \mathrm{C}$ by a calibrated mercury thermometer measured approximately the temperature of the water. Thermal electromotive forces were measured on a potentiometer with accessories such as to give a sensitivity of ro microvolts.

The venturi meter for measuring water flow was connected to a mercury $U$-tube gage and the meter and gage were calibrated together by weighing the water discharged in a given time at constant rates. A $3 \mathrm{~m}$ (Io foot) gravity head was used at first to produce water flow, but was replaced by the pump because it did not furnish high enough rates of flow. A flow of water through the cooling radiator in the return circuit of the wind tunnel was obtained by connecting to the water mains and was not measured.

53. OBSERVATIONS.--Care was taken to let the machinery run until temperatures were steady before taking readings. One observer read the electrical instruments measuring temperatures, while another observer read the gages and the hygrometer.

The quantities observed included the following: (I) Differential pressure between the pitot grid and its piezometer ring, (2) differential pressure between piezometer rings before and behind the radiator, (3) temperatures of wet and dry bulb hygrometer, (4) barometer pressure at the piezometer ring corresponding to the pitot grid, (5) temperature of the barometer, (6) differential, pressure of the venturi gage, (7) temperature of the venturi gage (8) resistance of the resistance thermometer, (9) e. m. f. of thermocouples for rise in air temperature, (ro) e. m. f. of thermocouples for drop in water temperature, (II) e. m. f. of reference couple for water temperature, (I2) temperature of the oil bath.

The complete calorimetric test of a section included the following sets of runs: (I) Atmospheric pressure, constant water flow, varying air flows; (2) constant air flow (mass flow), constant 
water flow, varying pressures, and air speeds; (3) constant pressure, constant air flow, varying water flow.

54. Computation.-Air flow was computed as explained in art. Io. Water flow was obtained directly from the readings of the venturi meter and a conversion factor depending upon the calibration constants and the units used. Heat dissipation was computed by two methods--heat received by the air and heat lost by the water- and the two measurements being independent furnished a good check on the observations. Temperatures were determined from the potentiometer readings for the thermocouples and their calibrations.

Using any consistent units, let

$M=$ air flow in mass per unit time per unit frontal area,

$C_{\mathrm{p}}=$ specific heat of air at constant pressure,

$W=$ water flow in mass per unit time,

$\theta=$ temperature of entering air,

$\theta_{2}=$ temperature of air on leaving the radiator,

$\theta^{\prime}=$ temperature of entering water,

$\theta^{\prime}{ }_{2}=$ temperature of water on leaving the radiator,

$\theta^{\prime}{ }_{m}=$ mean water temperature (taken as arithmetical mean),

$H_{1}=$ heat dissipation (water to air) per unit time per unit frontal area,

$\mathrm{H}_{2}=$ heat dissipation per unit time per unit frontal area per unit difference between mean water temperature and temperature of entering air,

$c_{1}, c_{2}, c_{3}, c_{4}=$ conversion factors, depending upon units used.

Heat received by the air is given in the two forms by the equations

$$
\begin{gathered}
H_{1}=c_{1} M C_{\mathrm{p}}\left(\theta_{2}-\theta\right) \\
H_{2}=c_{2} M C_{\mathrm{p}} \frac{\left(\theta_{2}-\theta\right)}{\left(\theta_{\mathrm{m}}^{\prime}-\theta\right)} .
\end{gathered}
$$

Heat lost by the water is given by

$$
\begin{aligned}
& H_{1}=c_{3} W\left(\theta_{2}{ }^{\prime}-\theta^{\prime}\right) \\
& H_{2}=c_{4} W \frac{\left(\theta_{2}{ }^{\prime}-\theta^{\prime}\right)}{\left(\theta^{\prime}{ }_{m}-\theta\right)} .
\end{aligned}
$$

The quantities which enter both expressions for $H_{2}$ are all given directly by the experimental data except $c_{2}, c_{4}, C_{\mathrm{p}}$, which are readily ascertained, and the quantity $\theta^{\prime}{ }_{m}$ which comes indirectly from the observed data by very simple arithmetic. 
(c) STEAM TUNNEL USING WATER

55. Adaptation to USE OF WATER.-The rebuilt steam tunnel differed from the one first built (described in art. 47), mainly in using the blower to draw air through the channel instead of pushing it through and in being equipped for the use of hot water as a source of heat. Water circulation was obtained by piping to this tunnel the water system of the reduced-pressure tunnel (art. $5^{2}$, Fig. 26). In the air system of the tunnel a bell mouth and a honeycomb at the intake were added to straighten the flow. Fig. 2 is a photograph of this wind tunnel as rebuilt, with the intake section moved back to show the mounting of the radiator.

Mercury thermometers in the tunnel before and behind the radiator and in the water line at inlet and outlet furnished a rough check on the measurement of heat dissipation. The temperature drop in the water was too small to be measured with accuracy by the thermometers, and the readings of the thermometer beyond the radiator were, in general, not reliable for anything better than a rough check. The governing computation of heat dissipation was accordingly made from the air flow and the rise in air temperature as indicated by the thermocouple grids, which were those used in the reduced-pressure tunnel (art. $5 \mathrm{I}$ ).

\section{B. HEAT TRANSFER, WATER OR STEAM TO METAL}

56. Governing Conditions. - The rate of transfer of heat from the circulating water to the metal walls of the water passages depends upon the temperature difference between water and metal, upon the rate of flow of water, and upon the degree of turbulence in the stream. The third factor depends upon the second, and the heat transfer per degree mean temperature difference is considerably less at very low rates of flow than at high rates. As the rate of flow is increased the corresponding increase in heat transfer per degree becomes less and less, and at the high rates of flow ordinarily used in aeronautic practice differences in the rate of flow have only neglible effects upon the transfer per degree.

Data showing in detail the effect of changes in rate of water flow are given in the curves of Appendix G (Figs. 128-137). The conclusion drawn from these data is that for all types of core commonly used any water flow exceeding 3 gallons per minute per inch depth of core (I foot wide) gives substantially the same heat dissipating performance of the core. With a flow so small as 2 gallons per minute per inch depth of core the heat dissipation is, in general, not lowered seriously, but below this rate of water flow 
the effect rapidly becomes more marked, as indicated by the curves to which reference is made. The numerical values relating to the conclusions stated above are exhibited in Table ro for many types of core. The column headed "Maximum heat dissipation" was obtained by estimating the positions of asymptotes to the curves forming Figs. I28-I37.

TABLE 10.-Relation of Heat Dissipation to Rate of Flow of Water

\begin{tabular}{|c|c|c|c|c|c|c|}
\hline \multirow{2}{*}{ Radi tor No. } & \multirow{2}{*}{$\begin{array}{l}\text { Depth of } \\
\text { core }\end{array}$} & \multirow{2}{*}{$\begin{array}{l}\text { Air flow } \\
\text { per unit } \\
\text { frontal } \\
\text { area }\end{array}$} & \multirow{2}{*}{$\begin{array}{l}\text { Maximum } \\
\text { heat } \\
\text { dissipation }\end{array}$} & \multicolumn{2}{|c|}{$\begin{array}{l}\text { Water filow of } 3 \text { gal- } \\
\text { lons per minute per } \\
\text { inch depth of core }\end{array}$} & \multirow{2}{*}{$\begin{array}{l}\text { (Water } \\
\text { flow of } \\
2 \text { gallons, } \\
\text { etc.) } \\
\text { Deficiency } \\
\text { from } \\
\text { maximum }\end{array}$} \\
\hline & & & & $\begin{array}{l}\text { Heat dis- } \\
\text { sipation }\end{array}$ & $\begin{array}{c}\text { Deficiency } \\
\text { from } \\
\text { maximum }\end{array}$ & \\
\hline A-23.. & $\begin{array}{c}\text { Inches } \\
5\end{array}$ & \begin{tabular}{r}
\multicolumn{1}{c}{ Lbs. } \\
$\mathrm{ft}^{2}$ \& sec. \\
2.04 \\
2.95 \\
4.10 \\
4.45
\end{tabular} & \begin{tabular}{|r|} 
hp. \\
ft. $.^{2} \times 100^{\circ} \mathbf{F}$ \\
19.0 \\
26.5 \\
33.7 \\
35.8
\end{tabular} & \begin{tabular}{r}
\multicolumn{1}{c|}{ hp. } \\
ft. $^{2} \times 100^{\circ} \mathrm{F}$ \\
18.5 \\
25.9 \\
33.0 \\
35.3
\end{tabular} & $\begin{array}{r}\text { Per cent } \\
3 \\
2 \\
2 \\
2\end{array}$ & $\begin{array}{r}\text { Per cent } \\
3 \\
4 \\
4 \\
2\end{array}$ \\
\hline B-3............. & 3 & $\begin{array}{l}2.63 \\
\text { 3. } 82 \\
5.12 \\
5.58\end{array}$ & $\begin{array}{l}12.7 \\
16.8 \\
20.4 \\
22.6\end{array}$ & \begin{tabular}{l|l|}
12.2 & \\
16.1 & 1 \\
19.8 \\
21.8
\end{tabular} & $\begin{array}{l}4 \\
4 \\
3 \\
4\end{array}$ & $\begin{array}{r}5 \\
8 \\
7 \\
10\end{array}$ \\
\hline B-5........... & 2 & $\begin{array}{l}\text { 2. } 42 \\
\text { 3. } 54 \\
4.89 \\
\text { 5. } 33\end{array}$ & $\begin{array}{l}11.6 \\
14.8 \\
17.6 \\
18.6\end{array}$ & $\begin{array}{l}11.2 \\
13.9 \\
16.7 \\
17.6\end{array}$ & $\begin{array}{l}4 \\
6 \\
5 \\
6\end{array}$ & $\begin{array}{r}6 \\
10 \\
8 \\
8\end{array}$ \\
\hline B-8.... & 5.3 & $\begin{array}{l}1.94 \\
2.86 \\
3.98\end{array}$ & $\begin{array}{l}\text { 18. } 0 \\
22.3 \\
28.8\end{array}$ & $\begin{array}{l}17.4 \\
21.8 \\
28.0\end{array}$ & $\begin{array}{l}3 \\
3 \\
3\end{array}$ & $\begin{array}{l}8 \\
6 \\
7\end{array}$ \\
\hline B-9........................ & 3. 5 & $\begin{array}{l}2.10 \\
\text { 3. } 12 \\
4.30 \\
4.80\end{array}$ & $\begin{array}{l}14.5 \\
20.3 \\
25.2 \\
29.0\end{array}$ & $\begin{array}{l}14.2 \\
19.4 \\
24.4 \\
28.0\end{array}$ & $\begin{array}{l}2 \\
4 \\
3 \\
3\end{array}$ & $\begin{array}{r}5 \\
10 \\
6 \\
7\end{array}$ \\
\hline $\begin{array}{c}\mathrm{C}-6 \ldots \\
,\end{array}$ & 3 & $\begin{array}{l}\text { 2. } 20 \\
3.38 \\
4.61 \\
5.07\end{array}$ & $\begin{array}{l}15.1 \\
20.7 \\
26.4 \\
28.2\end{array}$ & $\begin{array}{l}14.6 \\
20.2 \\
24.8 \\
26.7\end{array}$ & $\begin{array}{l}3 \\
3 \\
6 \\
6\end{array}$ & $\begin{array}{r}5 \\
4 \\
9 \\
10\end{array}$ \\
\hline D-1...... & 2. 7 & $\begin{array}{l}\text { 2. } 26 \\
\text { 3. } 45 \\
4.52 \\
\text { 5. } 00\end{array}$ & $\begin{array}{l}13.8 \\
18.2 \\
22.0 \\
23.8\end{array}$ & $\begin{array}{l}13.0 \\
17.2 \\
20.6 \\
22.4\end{array}$ & $\begin{array}{l}6 \\
6 \\
6 \\
6\end{array}$ & $\begin{array}{r}9 \\
8 \\
10 \\
9\end{array}$ \\
\hline F-5................. & 3.5 & $\begin{array}{l}1.60 \\
2.30 \\
3.18 \\
3.54\end{array}$ & $\begin{array}{l}15.7 \\
19.3 \\
23.4 \\
24.5\end{array}$ & $\begin{array}{l}15.0 \\
18.2 \\
22.1 \\
23.0\end{array}$ & $\begin{array}{l}4 \\
6 \\
5 \\
6\end{array}$ & $\begin{array}{l}8 \\
8 \\
9 \\
9\end{array}$ \\
\hline G-2.... & 1.7 & $\begin{array}{l}2.06 \\
\text { 3. } 02 \\
4.15 \\
4.52\end{array}$ & $\begin{array}{l}15.8 \\
20.5 \\
23.7 \\
25.5\end{array}$ & $\begin{array}{l}14.5 \\
18.7 \\
22.5 \\
23.6\end{array}$ & $\begin{array}{l}8 \\
9 \\
5 \\
8\end{array}$ & $\begin{array}{l}16 \\
15 \\
12 \\
11\end{array}$ \\
\hline G-4.............. & 4 & $\begin{array}{l}2.05 \\
3.44\end{array}$ & $\begin{array}{l}23.2 \\
33.2\end{array}$ & $\begin{array}{l}22.8 \\
32.6\end{array}$ & $\begin{array}{l}2 \\
2\end{array}$ & $\begin{array}{l}4 \\
4\end{array}$ \\
\hline
\end{tabular}

Accordingly, so long as the water flow is above a certain minimum, it is not of much importance how much above this minimum it is, except for the slight consideration which is merited by the following reason: The temperature of the water entering the radiator (leaving the engine cylinder jackets), rather than the mean 
temperature, must for operating conditions be maintained below a certain maximum, and an increase in rate of water flow may result in an increased heat transfer per degree difference between the metal and the entering water by raising slightly the mean temperature of the water. For example, if the water drops $20^{\circ}$ in temperature during its passage through the radiator at some high rate, doubling that rate would result in a drop of only about $10^{\circ}$, and the mean temperature would be raised $5^{\circ}$ for the same allowable maximum.

57. Comparison of Steam and Water as Sources of Heat FOR RADIATOR TESTS. - When steam is used in the radiator instead of water the heat dissipated to the air stream per degree temperature difference is found to be practically identical with that when water is used if the "mean" temperature of the steam and condensed water in the radiator is computed, as explained in article 50, by weighting each temperature according to the specific heat or heat of vaporization involved at that temperature. The differences obtained with ro types of radiator are shown in Table I I, compared in each case at the same air flow and with a high rate of water flow. Positive differences indicate that the heat dissipation was greater with steam than with water, and differences as high as 3 per cent are within the limit of experimental error. No reason for the larger differences has been discovered.

TABLE 11.-Comparison of Steam and Water as Sources of Heat in Radiators

\begin{tabular}{|c|c|c|c|}
\hline Radiator No. & $\begin{array}{c}\text { Average } \\
\text { differ- } \\
\text { ence in } \\
\text { heat dis- } \\
\text { sipation }\end{array}$ & Radiator No. & $\begin{array}{l}\text { Average } \\
\text { differ- } \\
\text { ence in } \\
\text { heat dis- } \\
\text { sipation }\end{array}$ \\
\hline$A-8, \ldots \ldots \ldots \ldots$ & $\begin{array}{r}\text { Per cent } \\
-2\end{array}$ & $\mathrm{C}-6 . . .$. & $\begin{array}{r}\text { Per cent } \\
+6\end{array}$ \\
\hline A-23................... & -1 & 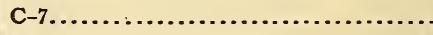 & +4 \\
\hline B-3........... & -3 & 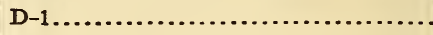 & +4 \\
\hline B-5................ & -5 & 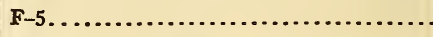 & -1 \\
\hline B-9................. & +2 & 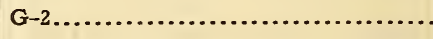 & -2 \\
\hline
\end{tabular}

C. HEAT TRANSFER THROUGH THE METAL

In the conduction of heat through the metal on its way from water to air the distinction between direct and indirect cooling surface is important.

58. Direct Cooling Surface.-For direct cooling surface, where water flows along one side of a thin metal sheet and air along the other side, the flow of heat is practically straight through the sheet, and only a small temperature difference between the 
two sides is needed to deliver heat as rapidly as it can be swept away from the surface by the air. The temperature drop ${ }^{21}$ in the metal is so small in comparison with the difference in temperature between air and water that the thickness and conductivity of the metal are of small importance in heat transfer. The composition and thickness of the metal will therefore be governed by such considerations as strength, weight, liability to corrosion, cost and ease of manufacture.

59. INDIRECT COOLING SURFACE.-For indirect cooling surface thickness and conductivity of the metal are of some importance. If before being dissipated to the air heat is conducted away from the water-tube walls along a "fin" projecting into the air stream, the distance through which much of the heat must be conducted is considerably greater than the thickness of the metal, and the difference in temperature between the tip of a fin and the watertube wall becomes of importance in lowering the mean temperature difference between air and metal. This reduction in temperature difference increases with increase in air speed, since as more heat is taken away from the surface of the fins a greater temperature difference is required to deliver the heat to the surface. The effectiveness of indirect cooling surface is considered below (art. 67).

\section{HEAT TRANSFER, METAL TO AIR}

The transfer of heat from metal to air depends upon temperature difference, speed and density of air, turbulence of air, and amount and character of cooling surface.

6o. Temperature DiFFERENCE. - The heat dissipation from each element of cooling surface is proportional to the difference in temperature between the surface and the air to which it transmits heat. It can be shown (Appendix E), however, that if the heat dissipation of the whole radiator is proportional to the mean temperature difference between air and water it is also proportional ${ }^{22}$ to the difference between the mean water temperature and the temperature of the air at entrance. If the water does not

\footnotetext{
21 Within any limits likely to be encountered in radiators. The magnitude of this temperature drop is consideredin Appendix D.

${ }^{22}$ Attention is directed to the fact that the relationship is proportionality and not identity. Heat dissipation expressed in terms of difference in average temperature of water and entrance temperature of air will obviously require a different number for its expression from the one to be used for a temperature difference obtained from average water and average air temperatures. The difference is not large, but is of such magnitude that the two methods of expression should be distinguished. The first form, which is used generally in this paper, is much the more convenient for intercomparisons of radiator core performance. The second form of expression is, however, necessary if the radiator is to be compared to any other form of heatdissipating device.
} 
change very much in temperature while passing through the radiator, an arithmetical mean of its temperatures on entering and on leaving the core will be sufficiently accurate for the mean water temperature. The difference between this mean and the temperature of the air at entrance is used throughout this report as the temperature difference under which the radiator operates.

6r. MASS FLow of AIR.-Measurements of heat dissipation in the reduced-pressure tunnel, with different speeds and densities of air, showed that for a given temperature difference and given radiator core the rate of heat dissipation is constant for constant mass flow of air, regardless of the particular combination of speed and density that give that air flow. The relation was tested for air densities ranging from atmospheric to a little less than half of atmospheric, corresponding to an altitude of about $7.5 \mathrm{~km}(25000$ feet), and is probably valid for somewhat lower densities. The results of the tests are shown in Table $\mathrm{I} 2$.

TABLE 12.-Relation of Heat Dissipation to Air Density

IMass of air passing through core per unit time maintained constant by changing velocity inversely as density; heat dissipation in horsepower per square foot frontal area for $100^{\circ} \mathrm{F}$ temperature difference between entrance air and water (average); air density in pounds per cubic foot; air flow in pounds per second per square foot frontal area.]

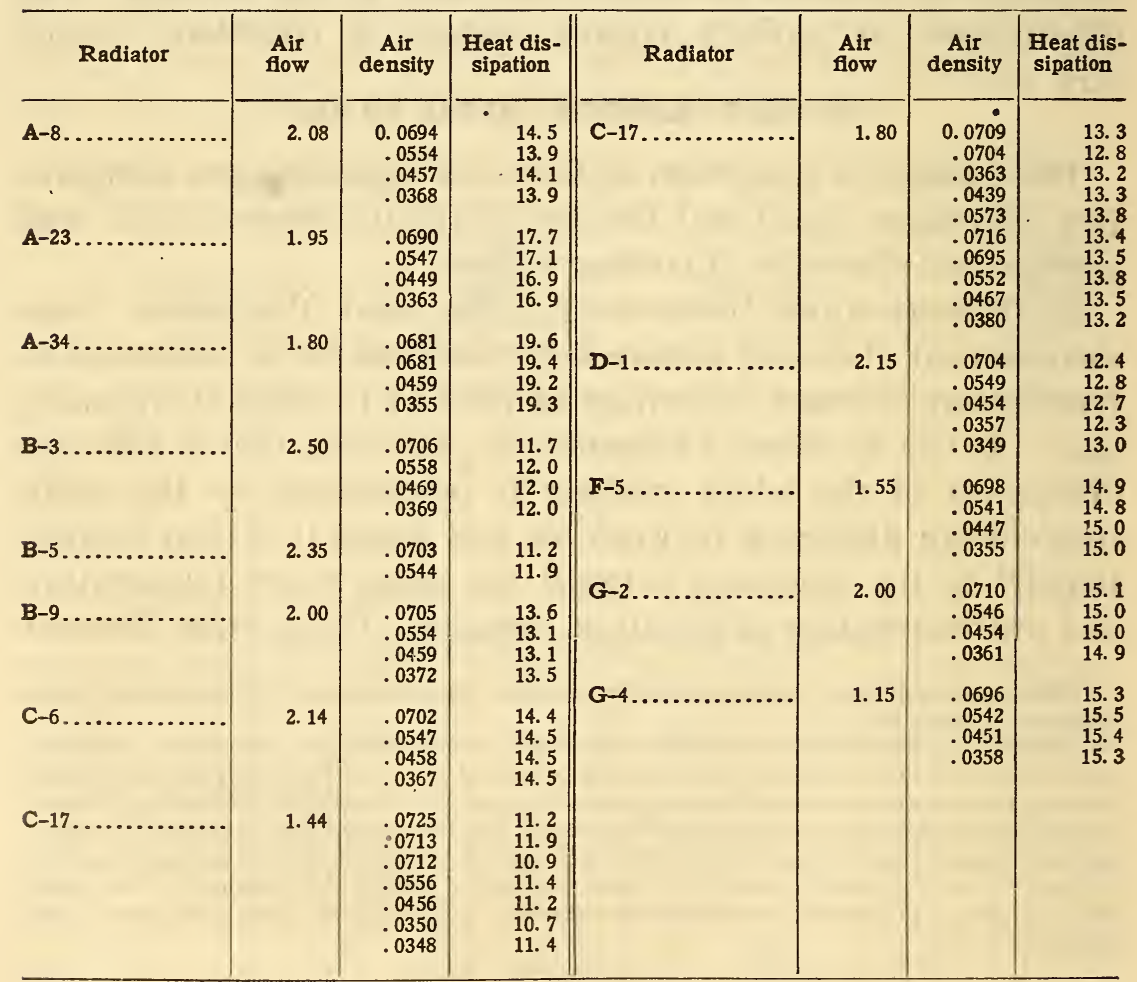


62. EMPIRICAL Equation FOR VARIation of Heat DissipaTION WITH AIR FLOW.-The heat dissipation for any particular type of core may be represented over a considerable range of air flow by an empirical equation with different constants for each type of core:

$$
H=b M^{\mathrm{n}} T
$$

where

$H=$ heat dissipation, metal to air, units of power per unit of frontal area,

$M=$ air flow, mass per unit time per unit frontal area,

$T=$ difference between temperature of the water (as a measure of that of the metal) and temperature of the entering air,

$b, n=$ constants for each type.

Values of $b$ and $n$ are given in Table $2 \mathrm{I}$.

It should be noted that a comparison of the values of $b$ for various types of core construction is worthless without a simultaneous consideration of $n$. The two constants go together in describing core performance, and the one alone is without interpretation.

63. Equation FOR VARIation of Heat Dissipation with DEPTH OF CORE.-An equation which may be used for estimating the heat dissipation of one type of core from that of a similar type of different depth may be derived as follows. Extending the above notation, let

$\theta=$ temperature difference between water and air, both at the same distance along the air tube from the front face, $\theta_{\theta}=$ value of $\theta$ at front face for any air tube,

$C_{\mathrm{p}}=$ specific heat of air at constant pressure,

$p=$ total perimeter of air tubes in unit frontal area around a section perpendicular to the direction of air flow,

$x=$ distance measured from front toward rear of radiator,

$x_{1}=$ depth of core,

$q=$ heat dissipation per unit surface per unit time per unit temperature difference.

Now, if it is assumed ( $\mathrm{I}$ ) that the transfer coefficient $q$ is independent of the distance $x$ from the front face, and (2) that the change in water temperature with depth of core is negligible in comparison with the temperature difference $\theta$, so that the change in $\theta$ in any one air tube may be regarded as due entirely to the 
rise in air temperature, then the heat gained by the air in passing an infinitesimal distance along the tubes may be placed equal to the heat dissipated by the surface past which the air has flowed, giving the equation

$$
-M C_{\mathrm{p}} d \theta=q \theta p d x
$$

which, making use of the fact that $\theta=\theta_{0}$ when $x=0$, integrates to the form

$$
\theta=\theta_{0} e^{-\alpha x}
$$

where $\alpha$ is defined by the relation

$$
\alpha=\frac{q p}{C_{\mathrm{p}} M} .
$$

The total heat transfer is given by the equation

$$
H=\int_{0}^{x_{1}} q \theta p d x=q p \theta_{0} \int_{0}^{x_{1}} e^{-\alpha \mathbf{x}} d x
$$

which integrates to the form

$$
H=\frac{q p \theta_{\mathrm{o}}}{\alpha}\left(1-e^{-\alpha x_{1}}\right)=\theta_{\mathrm{o}} C_{\mathrm{p}} M\left(1-e^{-\alpha x_{1}}\right) .
$$

The assumption that the change in the water temperature with depth of core is negligible in comparison with other factors of the problem is equivalent to replacing $\theta_{0}$ by $T$ when summing the values of $\theta_{0}$ over the whole cluster of tubes and averaging

$$
H=C_{\mathrm{p}} M T\left(1-e^{-\alpha \mathrm{x}_{1}}\right) .
$$

The coefficient $\alpha$ is not a constant, even for a particular radiator, because the surface dissipation coefficient $q$ varies with the air flow; but from a single measurement of heat dissipation a value of $\alpha$ may be computed by equation (7) and may then be used to estimate heat dissipation at the same air flow in a similar type of core of different depth. An indication of the accuracy of this method of estimating heat dissipation may be obtained by comparing the values of the coefficient for types of radiator differing only in depth, and since for similar types $\alpha$ is by definition (equation 4) proportional to the heat dissipation coefficient $q$, comparisons may equally well be made by means of $q$. In Table I3 values of $q$ taken from Table 2 I are shown for groups of radiators that are nearly alike except in depth. Reference to Table 20 will show that the geometrical characteristics. are not in every case quite the same, so that the validity of the equation as a change of 
depth relation is probably a little better than the table indicates. An examination of equation (7) has shown that over the range of values covered a difference of a certain per cent in $\alpha$ (or $q$ ) introduces a difference of very nearly the same per cent in $H$.

TABLE 13.-Comparison of Coefficients q, Depths of Core Varying

\begin{tabular}{|c|c|c|c|c|c|c|c|}
\hline \multirow{2}{*}{$\begin{array}{c}\text { Radiator } \\
\text { No. }\end{array}$} & \multicolumn{2}{|c|}{ Depth } & \multirow{2}{*}{$\begin{array}{c}q \times 1000 \\
\left(\frac{\text { cal. }}{\mathrm{cm}^{2} \times \mathrm{sec} . \times{ }^{\circ} \mathrm{C}}\right)\end{array}$} & \multirow{2}{*}{$\begin{array}{c}\text { Radiator } \\
\text { No. }\end{array}$} & \multicolumn{2}{|c|}{ Depth } & \multirow{2}{*}{$\begin{array}{c}q \times 1000 \\
\left.\frac{\text { cal. }}{\mathrm{m}^{2} \times \text { sec. } \times{ }^{\circ} \mathrm{C}}\right)\end{array}$} \\
\hline & $\mathrm{cm}$ & Inches & & & $\mathrm{cm}$ & Inches & \\
\hline 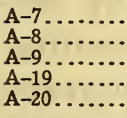 & $\begin{array}{r}12.3 \\
6.0 \\
7.2 \\
7.6 \\
12.7\end{array}$ & $\begin{array}{l}\text { 4. } 9 \\
2.4 \\
2.8 \\
\text { 3. } 0 \\
5.0\end{array}$ & $\begin{array}{l}\text { 3. } 2 \\
\text { 3. } 3 \\
\text { 3. } 6 \\
\text { 3. } 1 \\
\text { 3. } 1\end{array}$ & $\begin{array}{l}\text { B-12...... } \\
\text { B-13...... } \\
\text { B-14 } \\
\text { B-15........ } \\
\text { B-16...... }\end{array}$ & $\begin{array}{r}8.3 \\
10.2 \\
7.6 \\
10.1 \\
10.1\end{array}$ & $\begin{array}{l}\text { 3. } 3 \\
\text { 4. } 0 \\
\text { 3. } 0 \\
4.0 \\
\text { 4. } 0\end{array}$ & $\begin{array}{l}\text { 3. } 4 \\
\text { 3. } \\
\text { 3. } \\
\text { 3. } 1 \\
3.0\end{array}$ \\
\hline $\begin{array}{l}A-21 \ldots \ldots . \\
A-22 \ldots \ldots \\
A-23 \ldots \ldots \\
B-5 \ldots \ldots \\
B-6 \ldots \ldots\end{array}$ & $\begin{array}{r}7.5 \\
10.1 \\
12.7 \\
5.2 \\
8.3\end{array}$ & $\begin{array}{l}\text { 3. } 0 \\
\text { 4. } 0 \\
\text { 5. } 0 \\
\text { 2. } 0 \\
\text { 3. } 3\end{array}$ & $\begin{array}{l}\text { 3. } 0 \\
\text { 3. } 0 \\
2.9 \\
2.9 \\
2.6\end{array}$ & $\begin{array}{l}\mathbf{C}-8 \ldots \ldots \\
\mathbf{C}-12 \ldots \\
\mathbf{C}-13 \ldots \\
\mathrm{C}-9 . \ldots \\
\mathbf{C}-10 \ldots \\
-\end{array}$ & $\begin{array}{l}12.8 \\
25.3 \\
20.3 \\
10.2 \\
10.2\end{array}$ & $\begin{array}{r}5.0 \\
10.0 \\
8.0 \\
4.0 \\
4.0\end{array}$ & $\begin{array}{l}3.1 \\
3.0 \\
3.2 \\
3.1 \\
3.3\end{array}$ \\
\hline 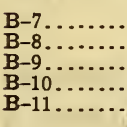 & $\begin{array}{r}10.2 \\
13.4 \\
8.9 \\
6.4 \\
7.6\end{array}$ & $\begin{array}{l}\text { 4. } 0 \\
\text { 5. } 3 \\
\text { 3. } 5 \\
\text { 2. } 5 \\
\text { 3. } 0\end{array}$ & $\begin{array}{l}\text { 2. } 7 \\
2.3 \\
\text { 3. } 0 \\
\text { 3. } 6 \\
\text { 3. } 5\end{array}$ & $\begin{array}{l}\text { C- }-11 \ldots \ldots \\
\text { C-14....... } \\
\text { G-2 } 2 \ldots \ldots \ldots \\
\text { G-3....... }\end{array}$ & $\begin{array}{r}10.2 \\
20.3 \\
4.3 \\
8.6\end{array}$ & $\begin{array}{l}4.0 \\
8.0 \\
1.7 \\
3.4\end{array}$ & $\begin{array}{l}3.4 \\
\text { 3. } \\
4.5 \\
4.5\end{array}$ \\
\hline
\end{tabular}

In using equation (7) as a basis for computing $q$ from calorimetric measurements of radiator cores it is worth while to notice that the equation contains explicitly as a temperature factor the difference between the temperatures of the entrance air and average water. Accordingly, values of $H$ may be taken directly in the form in which they are expressed elsewhere in this paper, namely, for a given temperature difference, usually $100^{\circ} \mathrm{F}$ or $50^{\circ} \mathrm{C}$ between entrance air and average water. When the conditions permit of coordinating average air temperature and average water temperature with respect to the entire cooling surface of a core, the computation of $q$ directly from its definition is much simpler than from equation (7), avoiding the complicated arithmetic of the exponential term. The heat dissipation corresponding to any given air flow furnishes the value of the approximate rise in temperature of the air. For a simple core construction and most of the cooling surface direct a fair approximation is to assume the temperature gradients linear, and that the heat dissipation is given by considering all the metal at the average water temperature and all the air at the average air temperature, both averages being arithmetic means of entrance and exit temperatures. The total heat dissipation divided by the difference in these two average temperatures and by the total cooling surface is the value of $q$. 
Core $\mathrm{C}-\mathrm{I}_{3}$, a circular cell construction with water surrounding every air tube, might be expected to fulfill very closely the assumptions stated above regarding uniformity of temperature and temperature gradients. The value of $q$ computed from the heat dissipation for an air flow corresponding to a speed of $30 \mathrm{~m}$ per $\sec ($ Ioo feet per sec) through the air tube is 3.15 , while the value from the exponent of the integrated equation (7) is 3.18. So nearly perfect a check is to be expected only with most favorable conditions of core construction, but for other cores it is readily possible to ascertain the approximate value of $q$, probably generally within 5 to 15 per cent by the short method of computing.

64. Relation Between Heat Dissipation and Surface FRICTION.-For estimating the heat dissipation entirely from the geometrical characteristics of a core an approximate value of the coefficient $\alpha$ may be obtained, which applies fairly well to types with straight, smooth-walled tubes.

It has been pointed out by Lanchester and Stanton ${ }^{23}$ that an approximate value of the surface heat dissipation coefficient $q$ is given by the relation

where

$$
\text { - } q=\frac{C_{\mathrm{p}} R_{\mathrm{f}}}{v}
$$

$$
\begin{aligned}
R_{\mathrm{f}} & =\text { surface friction force per unit surface, } \\
v & =\text { linear velocity of air in tubes. }
\end{aligned}
$$

Surface friction may be estimated from the formula of C. $\mathrm{H}$. Lees ${ }^{24}$ for smooth, round tubes.

$$
R_{\mathrm{f}}=\rho v^{2}\left[0.0765\left(\frac{\mu}{v \rho D}\right)^{0.35}+0.0009\right]
$$

where

$$
\begin{aligned}
\rho & =\text { density of air } \\
\mu & =\text { viscosity of air, } \\
D & =\text { diameter of tube. }
\end{aligned}
$$

Substituting equation (2) in equation (I) and equation (I) in equation (4) of article 63 , which defines $\alpha$, gives for the exponent $\alpha x_{1}$ of equation (7)

$$
\alpha x_{1}=\frac{p \rho v x_{1}}{M}\left[0.0765\left(\frac{\mu}{v \rho D}\right)^{0.35}+0.0009\right]
$$

${ }^{23}$ Great Britain Committee for Aeronautics Technical Report, I9I2-13, p. 45.

24 See art. 32 . 
The physical quantities enter in a dimensionless connection, hence the equation holds for any consistent units. Making use of the relation that mass flow of air per unit time is the simple function of density and velocitv of the air,

and

$$
M=a \rho v
$$

where

$$
D=4^{r}
$$

$$
\begin{aligned}
& a=\text { free area of core, } \\
& r=\text { hydraulic radius of air tubes, }
\end{aligned}
$$

equation (3) may be put in the form

$$
\alpha x_{1}=\frac{p x_{1}}{a}\left[0.047 \mathrm{I}\left(\frac{a \mu}{r M}\right)^{0.35}+0.0009\right] \text {. }
$$

For surface which is all direct the factor $\frac{p x_{1}}{a}$ may be simplified still further, thus: Since $a$, the free area in unit frontal area, is $A_{1}+A_{2}+\cdots+A_{n}$, the sum of the areas of the individual air tubes, and since area is perimeter times hydraulic radius, $a=p_{1} r+p_{2} r+\cdots+p_{\mathrm{n}} r$, when the tubes are alike and $r$ is the average hydraulic radius. But the sum of $p_{1}+p_{2}+\cdots+p_{n}$, the perimeters of the tubes in unit frontal area, is $p$. Hence, $p=a / r$ and $p / a=\mathrm{I} / r$.

Equation (4) gives the exponent of equation (7) (art. 63) in terms of the geometrical characteristics of the core, the viscosity of the air, and the air flow. By using hydraulic radius rather than diameter of tube the equation is made applicable to square and hexagonal tubes.

Equation (I) with the exponent indicated by equation (4) is applicable to types of core with direct cooling surface only, and when there is any considerable amount of indirect cooling surface a correction may be made in accordance with articles 68 and 69. When so corrected, the agreement between heat dissipation as computed and as actually measured is shown in Table I4. 
TABLE 14.-Comparison of Observed and Computed Heat Dissipation

[Air flow 4 pounds per second per square foot frontal area of core]

\begin{tabular}{|c|c|c|c|c|c|}
\hline \multirow{2}{*}{ Radiator No. } & \multicolumn{2}{|c|}{$\begin{array}{l}\text { Heat dissipation } \\
\left(\frac{\mathrm{hp}}{\mathrm{ft} .^{2} \times 100^{\circ} \mathrm{F}}\right)\end{array}$} & \multirow{2}{*}{ Radiator No. } & \multicolumn{2}{|c|}{$\begin{array}{l}\text { Heat dissipation } \\
\left(\frac{\mathrm{hp}}{\mathrm{ft} .^{2} \times 100^{\circ} \mathrm{F}}\right)\end{array}$} \\
\hline & $\begin{array}{l}\text { Com- } \\
\text { puted }\end{array}$ & Observed & & $\begin{array}{l}\text { Com-- } \\
\text { puted }\end{array}$ & Observed \\
\hline $\begin{array}{l}\text { A }-1 \\
A-2 \\
A-3 \\
A-1 \\
A-4 \\
A-5\end{array}$ & $\begin{array}{r}31 \\
38 \\
21 \\
19 \\
27\end{array}$ & $\begin{array}{l}33 \\
37 \\
20 \\
22 \\
27\end{array}$ & 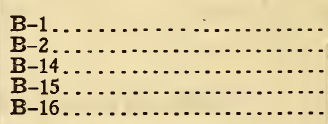 & $\begin{array}{l}15 \\
21 \\
20 \\
26 \\
26\end{array}$ & $\begin{array}{l}15 \\
19 \\
20 \\
25 \\
25.5\end{array}$ \\
\hline 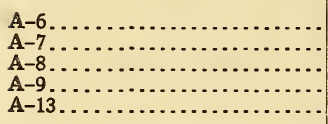 & $\begin{array}{l}33 \\
46 \\
24 \\
29 \\
32\end{array}$ & $\begin{array}{l}31 \\
43 \\
23 \\
30 \\
27\end{array}$ & $\begin{array}{l}\text { C }-1 \ldots \ldots \\
\text { C-4 } \\
\text { C-5 } \\
\text { C-8 } \\
\text { C-9.1. }\end{array}$ & $\begin{array}{l}28 \\
25 \\
30 \\
26 \\
26\end{array}$ & $\begin{array}{l}24 \\
23 \\
29 \\
26 \\
26\end{array}$ \\
\hline 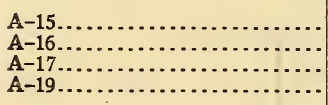 & $\begin{array}{l}44 \\
25 \\
32 \\
30\end{array}$ & $\begin{array}{l}42 \\
23 \\
28 \\
28\end{array}$ & 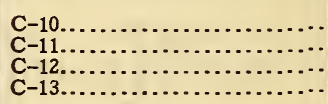 & $\begin{array}{l}26 \\
25 \\
44 \\
39\end{array}$ & $\begin{array}{l}26 \\
26 \\
45 \\
39\end{array}$ \\
\hline $\begin{array}{l}\mathrm{A}-20 \\
\mathrm{~A}-21 \\
\mathrm{~A}-22 \ldots \\
\mathrm{A}-23 \ldots \ldots\end{array}$ & $\begin{array}{l}47 \\
23 \\
30 \\
37\end{array}$ & $\begin{array}{l}43 \\
21 \\
28 \\
33\end{array}$ & 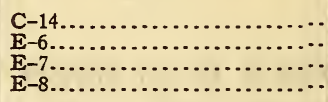 & $\begin{array}{l}50 \\
50 \\
30 \\
21\end{array}$ & $\begin{array}{l}50 \\
49 \\
30 \\
23\end{array}$ \\
\hline
\end{tabular}

Computed values from, $H=C_{\mathrm{p}} M T\left(\mathrm{I}-e^{-\alpha x_{1}}\right)$

$$
\alpha x_{1}=\frac{x_{1}}{r}\left[0.047 \mathrm{I}\left(\frac{a \mu}{r M_{i}}\right)^{0.35}+0.0009\right]
$$

$H=$ heat dissipated (h. p.) per unit frontal area (sq. ft.).

$C_{\mathrm{p}}=$ specific heat of air at constant pressure (h. p. sec per $\mathrm{lb}$. per ${ }^{\circ} \mathrm{F}$.).

$M=$ mass flow of air in pounds per second per unit frontal area. $\mathrm{M}=4$.

$T=$ temperature difference, water and air at entrance. $T=100^{\circ} \mathrm{F}$.

$x_{1}=$ depth of air tubes.

$r=$ hydraulic radius of air tube, measured in same unit of length (any unit).

$\mu=$ viscosity of air, expressed in a unit consistent with $M$ and $r$. $a=$ free area (a ratio, no unit).

Forindirect surface a correction to $x_{1} / r$ enters (see bottom p. $32 \mathrm{I}$ ). 65. Amount of Cooling Surface.-In general, increasing the amount of cooling surface (per unit frontal area) increases the heat dissipation, but if a core is redesigned with greater cooling surface the increase in heat dissipation is not, in general, proportional to the increase in the surface. Because of the rise in temperature of the air in passing through the radiator and the consequent. 
decrease in temperature difference between air and water, surface added by increasing the depth of the core becomes less and less effective in heat dissipation as the depth increases. If surface is added by making the air tubes smaller, the condition of turbulence in the air may be changed, and, in general, the change seems to be such as to cause an increase in the heat dissipation per unit cooling surface. Indirect cooling surface is less effective than direct, because of the drop in temperature along the metal. Spiral vanes are sometimes placed in the air tubes and furnish additional surface which, although entirely indirect, tends to increase the heat dissipation per unit surface by mixing the air stream. Whether additional surface is obtained by increase in depth, by decrease in size of air passages, or by addition of vanes, it adds to air resistance and, although increasing heat dissipation for a given air flow through the core, may decrease the air flow to such an extent that for a given flying speed nothing is gained, while much is lost because of a great increase in head resistance.

Differences either in nature or degree of turbulence of the air are indicated by the varying values of the surface cooling coefficient $q$ of Table $2 \mathrm{r}$. These values are computed from equation (7) (art. 63) for such an air flow as gives a linear speed of $30 \mathrm{~m}$ per sec (roo feet per second) through the air tubes.

A special effect of turbulence in the air is shown in the type $\mathrm{C}-2 \mathrm{~b}$, which has approximately circular air tubes $20 \mathrm{~cm}$ ( 8 inches) long, with spiral vanes placed in one end extending for $5 \mathrm{~cm}(2$ inches) into the tube. When the section is turned so that the vanes are in front, the heat dissipation is about ro per cent greater for a given air flow than when they are in the rear end of the tube. The difference is shown in Fig. 27.

66. Condition of Cooling Surface.-As a general physical relation smoothness of cooling surface and its rate of heat dissipation bear too close a connection to neglect the former in considering its effects on the latter, although, as will be shown below, the differences in smoothness of metal in radiator tubes seem to fall within a range where the effect is not appreciable. A very highly polished surface shows a greater rate of heat transfer to a very high velocity air stream ${ }^{25}$ than does one only ordinarily smooth, and in a tube the polish not only facilitates heat transfer at a given air flow but allows an increase in air flow. Conse-

25 The fact that a high polish facilitates heat transfer at comparatively high rates of air flow past the surface should not be confused with the fact that a high polish is desirable for low heat transfer in cases where the transfer is largely due to radiation, such as the surfaces of thermos bottles. 
quently, in a radiator tube this would result in a further increase in heat dissipation at a given flying speed. Surfaces usually found in radiators, however, do not differ enough in smoothness to show any very marked effect. The sections $\mathrm{C}-8$ and $\mathrm{C}-9$ were tested for heat dissipation both with very rough surfaces and after the surfaces had been somewhat smoothed, but the difference in heat dissipation at constant air flow was within the limit

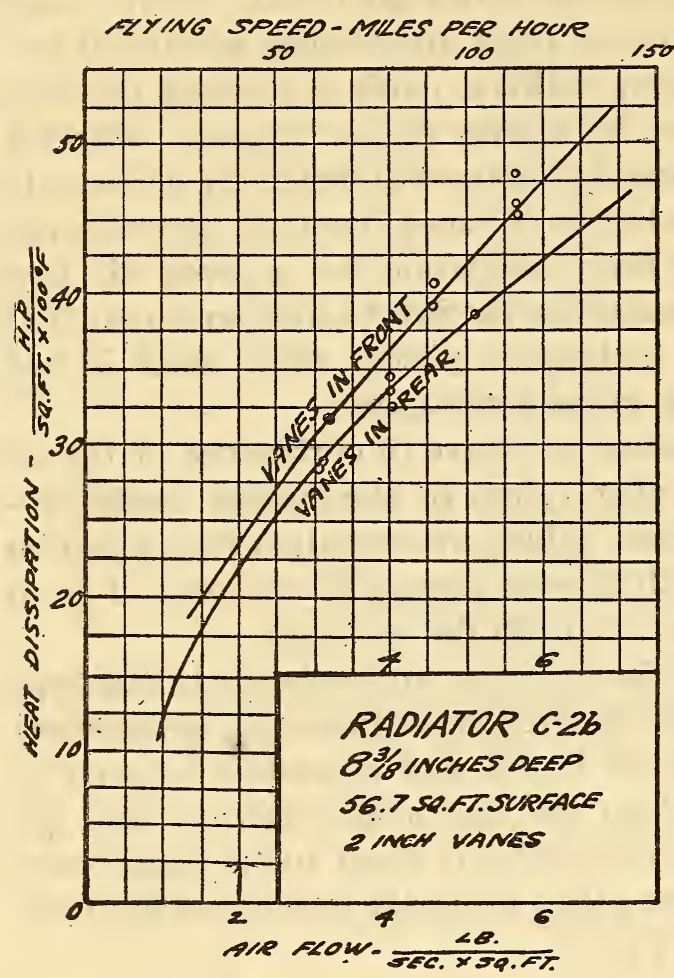

FIG. 27.-Heat dissipation for radiator core provided with spiral vanes near one face to increase the turbulence of the air flow

Considerable difference in turbulence and thus in heat dissipation is found according to whether or not the air stream strikes these spiral vanes immediately upon entering the core

Eight thermocouples were soldered into of experimental error, although the gain in air flow due to smoothing the surface (art. 2I) was enough to increase the heat dissipation about 5 per cent at constant flying speed. Comparisons at constant air flow are shown on Fig. 28.

Cleanness of cooling surface is very important. The accumulation of oil and dust sometimes found in radiator air tubes reduces the heat dissipation very greatly. A more detailed study of the effect of conditions of surface on heat dissipation was made with a single brass tube $4 \mathrm{I} .5 \mathrm{~cm} \quad$ (I6.3 inches) long, with an inside diameter of $7.8 \mathrm{~mm}$ (0.3 I inch), and with walls approximately I $\mathrm{mm}$ (o.04 inch) thick. side shets on the outside of the tube at points $2,7, \mathrm{I} 2$, and $17 \mathrm{~cm}$ from the ends and heat was supplied electrically from a coil of No. 32 copper wire wound closely the entire length of the tube and insulated with baked shellac. The tube was wrapped in hair felt with a corrugated paper covering to within $\mathrm{I} \mathrm{cm}$ of each end, and the ends were wrapped with several layers of friction tape and inserted tightly into two wooden boxes or chambers in which the 
properties of the air could be measured as it entered and left the tube. These chambers were $7.6 \mathrm{~cm}$ (3 inches) square and 15.2 $\mathrm{cm}$ (6 inches) long, and each was divided into three compartments by screens. The air entering the first compartment of the inlet chamber passed through a series of screens of coarse mesh wire and finally through a thin screen of hair felt into the second compartment, where its temperature was measured. It then passed through another screen into a third compartment, into which the end of the tube projected about I $\mathrm{mm}$ (0.04 inch). This compartment was connected to one side of a vertical oil gage used to measure the pressure drop through the tube. On leaving the tube the air passed through the first compartment of the exit chamber, which was connected to the other side of the oil gage, and then through a screen of wire and a layer of hair felt into the thermometer section, and finally through another screen into the last compartment, which was connected to the inlet of the fan. The exit chamber was very carefully lagged with $2.5 \mathrm{~cm}$ of cork on the outside, also with $0.5 \mathrm{~cm}$ of hair felt on the inside, to prevent the turbulent air from striking the wooden walls directly.

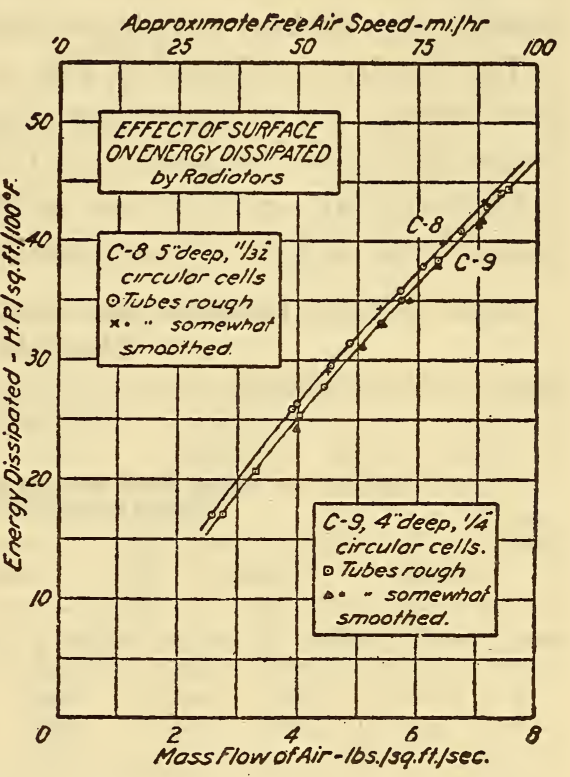

Fig. 28.-Effect of roughness of surface on heat dissipation

Neither radiator core shows any appreciable difference in heat dissipation at a given air flow for the two conditions of very rough and moderately smooth air tubes. At a given flying speed a small but appreciable difference would be in evidence, due to effect of roughness on the air flow (Art. 21, also Fig. 20)

The mass of air flowing through the tube was computer from the heat input to the coil, the rise in temperature of the air, and the specific heat of the air-that is, by using the tube itself as a Thomas meter-with the exception that pressure drop through the tube was used as the air meter for some of the runs after the pressure drop had been calibrated against the tube as a Thomas meter. The pressure-drop method was used in some of the earlier runs when mercury thermometers were used to measure the tcmperature rise, but was abandoned when thermocouples were used $75013^{\circ}-22-6$ 
for this measurement. The thermocouples were read on a "pyrovolter," and the heat input to the coil was obtained from readings of a voltmeter and an ammeter. Care was taken to obtain steady temperature conditions before beginning any set of readings.

Five conditions of service were used, namely: (I) Original surface (as the tube was drawn), (2) surface polished (considerable time and effort were expended in getting a high degree of polish), (3) polished surface lightly oiled, (4) polished surface lightly smoked, (5) surface roughened with fine sandpaper.

The results are shown in Fig. 29 and in Table I5, which shows heat dissipation in watts per degree centigrade of difference between the mean temperature of the tube and the temperature of the entering air and per cent of increase or decrease of heat dissipation over that for the original surface.

TABLE 15.-Heat Dissipation from Inside Wall of a Cylindrical Tube to High Velocity Air Stream

[Tube $0.78 \mathrm{~cm}$ inside diameter; air flow of 1 gram per second corresponds to approximate air speed 17.5 meters per second.]

\begin{tabular}{r|r|r|r|r|r|r|r|r}
\hline \multirow{2}{*}{$\begin{array}{c}\text { Air } \\
\text { flow }\end{array}$} & \multicolumn{6}{|c|}{ Heat dissipated from surface in five different conditions; also percentage differences from value for } \\
original surface (that of drawn brass tubing)
\end{tabular}

The table shows the following: (I) The highly polished surface dissipated about $\mathrm{I} 7$ per cent more heat (at the higher speeds) than one fairly rough, (2) the smoked surface dissipated about ro per cent less heat than the fairly rough one, (3) oiling the polished surface had very little effect on its heat dissipation, (4) the roughened surface was not much different in its heat dissipating qualities from the original surface.

The considerable difference in heat dissipation between the fairly rough and the highly polished surfaces may be accounted for by the fact that roughness allows a blanket of more or less stagnant air to cling to the surface, and thereby to some extent prevents the scouring of the surface that is required for the most rapid transfer of heat. The polishing was carried to an extreme entirely impracticable for radiator manufacture, and the results of the measurement may be considered to confirm those of Fig. 28, 
namely, no appreciable ( \pm 3 per cent) differences in heat dissipation for roughness and smoothness as ordinarily considered in radiator surfaces.

67. INDIRECT COOLING SURFACE.-Cooling surface not backed by flowing water, called "indirect cooling surface," is less effective per unit area in heat dissipation than direct surface, but can often be used to good advantage if proper attention be given to thermal conductivity, thermal contact between the indirect surface and the water-tube walls, and shortness of the distances over which heat must be conducted through the fins. The use of indirect cooling surface amounts to closing water tube by pressing the walls together, or to substituting a single sheet of metal for a water tube (compare Fig. 62 to Fig. $6 \mathrm{I}$ or Fig. 60 , and Fig. 83 to 84 ). The advantages are in saving the weight of water contained in the tube, and in the latter case the weight of one wall, while allowing relatively large free area of

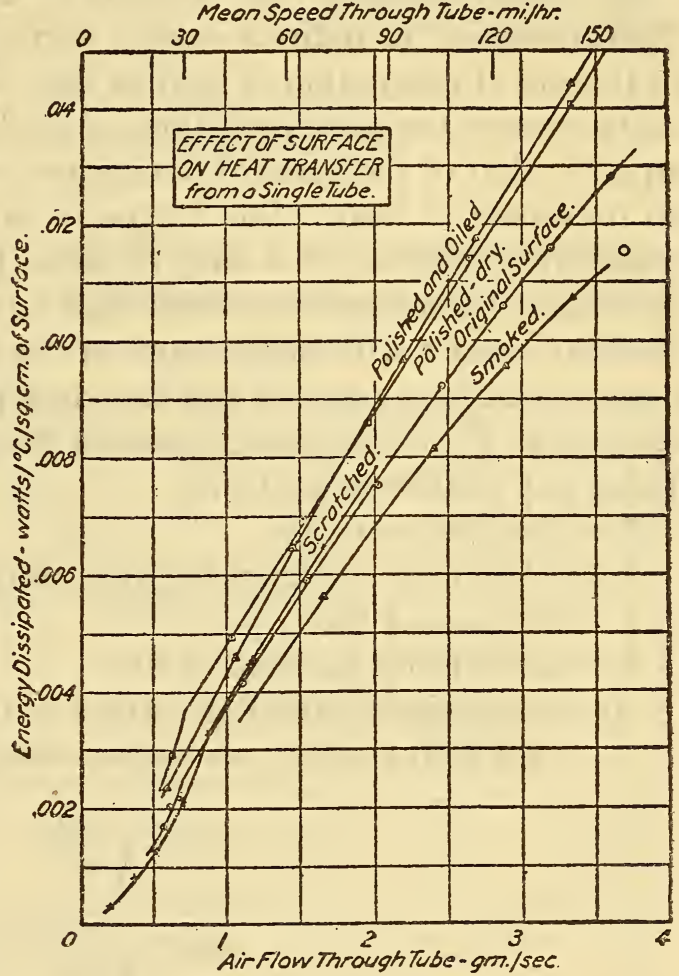

FrG. 29.-Effect of condition of surface on heat dissipated from inside wall of a single long tube

For the air flow corresponding to a linear velocity of 60 miles per hour in the tube (for which the flying speed of a radiator tube would be greater) the heat dissipation is increased !by I $_{5}$ per cent by polishing the original tube surface very highly and is decreased ro per cent by roughening it with a smoke deposit core for a given area of coiling surface. The disadvantage arises from the fact that heat must be transferred by metallic conduction for a distance along the fins and away from the water-tube walls before being dissipated to the air. Since heat can be conducted along the fins only with a drop in temperature, the temperature difference between metal and air is less for fins than for direct surface and decreases with distance from the source of heat. The temperature difference 
obviously decreases with increase in air flow, since heat dissipation increases with air flow, and greater temperature drop in the metal will be required to deliver heat to the surface as fast as it is taken up by the air. It is evident that good thermal contact is essential between the indirect cooling surfaces and the watertube walls from which they draw heat.

68. EFFECTIVENESS OF INDIRECT COOLING SURFACE.-The "effectiveness" of indirect cooling surface is defined as the ratio of its rate of dissipation of heat to that of an equal area of direct surface under the same conditions of air flow, but at a temperature equal to that of the parts of the indirect surface that are adjacent to its source of heat. For a type of fin often found in cellular radiators, consisting of a strip of metal lying between two water tubes and extending from front face to rear face of radiator, in thermal contact with one or both of the water tubes-i. e., along one or both long edges of the fin-an equation has been derived (Appendix F) for the effectiveness of the fin.

Using any consistent units, let

$E=$ effectiveness of fin,

$b=$ width of fin (distance between water tubes),

$y=$ thickness of fin,

$k=$ conductivity of metal of fin,

$q=$ heat dissipated from fin surface in units of heat per unit time, per unit surface, per degree temperature difference.

$$
\begin{aligned}
& u_{1}=\frac{b}{2} \sqrt{\frac{2 q}{y k}} \\
& u_{2}=b \sqrt{\frac{2 q}{y k}} .
\end{aligned}
$$

The effectiveness is found to be equal to

$$
E=\frac{\tanh u}{u}
$$

where $u$ takes the respective values $u_{1}$ and $u_{2}$ for the case of good thermal contact along both edges of fin and the case of good thermal contact along only one edge of fin. The quantity $u$ is dimensionless, so that any consistent units may be used in its computation. Values of the ratio $\frac{\tanh u}{u}$ are shown for different values of $u$ on Fig. 30.

69. Computation of Heat Dissipation for Surface Partli Direct AND PARTLy INDIRECT.-Differences in total heat dissi- 
pated by a radiator due to differences in amount and effectiveness of indirect cooling surface may be computed as follows:

Let

$H=$ heat dissipation of radiator,

$H_{\mathrm{d}}=$ heat dissipated by the direct cooling surface,

$H_{\mathrm{i}}=$ heat dissipated by the indirect cooling surface,

$H_{\mathrm{o}}=$ heat dissipation which the radiator would show if all the cooling surface were direct, or if the effectiveness of the indirect surface were I.oo,

$r=$ ratio of indirect to total cooling surface,

$r^{\prime}=$ ratio of indirect to direct cooling surface,

$E=$ effectiveness of the fins.

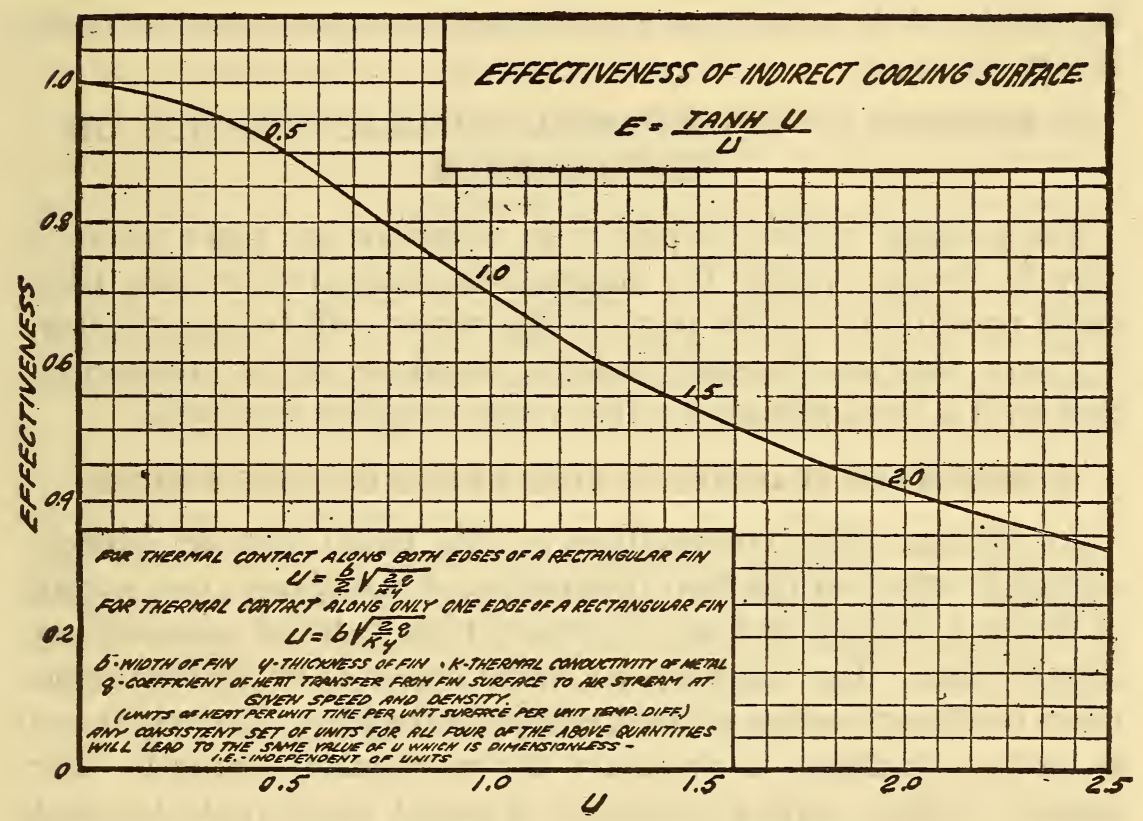

Fig. 30.-Effectiveness of indirect cooling surface

Ratio of heat dissipation by such surface to the dissipation by an equal area of direct surface under the same conditions of air flow but all at a temperature equal to the temperature of that portion of the indirect surface adjacent to its source of heat.

For indirect cooling surface supplied by a rectangular fin, the function $U$ which is the abscissa of the plot is determined by the geometrical and thermal constants of the fin in the simple formula shown in the figure

Now

and

$$
H=H_{\mathrm{d}}+H_{\mathrm{i}}
$$

so that

$$
H_{\mathrm{i}}=E r^{\prime} H_{\mathrm{d}}
$$

Also

$$
H=H_{\mathrm{d}}\left(\mathrm{I}+E r^{\prime}\right)
$$

$$
\text { (if } E=\mathrm{I} \text { ), } H_{\mathrm{o}}=H_{\mathrm{d}}\left(\mathrm{I}+r^{\prime}\right) \text {. }
$$


Then, the ratio of heat dissipated by the radiator to that which would have been dissipated were all the surface direct is

Substituting

$$
\frac{H}{H_{\mathrm{o}}}=\frac{\mathrm{I}+E r^{\prime}}{\mathrm{I}+r^{\prime}}
$$

$$
\begin{aligned}
r^{\prime} & =\frac{r}{\mathrm{I}-r} \\
\frac{H}{H_{\mathrm{o}}} & =\frac{\mathrm{I}+E \frac{r}{\mathrm{I}-r}}{\mathrm{I}+\frac{r}{\mathrm{I}-r}}=\mathrm{I}-(\mathrm{I}-E) r
\end{aligned}
$$

An assumption of indirect cooling surface as high as 60 per cent of the total and the effectiveness as low as 90 per cent would give for this ratio 0.94 .

\section{EFFECTS OF GEOMETRICAL CHARACTERISTICS ON PERFORMANCE}

The purpose of this chapter is to collect in one place where it may be found readily the information indicated by the title. Some repetition of other parts of the report will be unavoidable, but items that are discussed in detail elsewhere will be summarized here with a cross reference to the more complete discussion.

\section{A. EFFECTS OF FEATURES OF CORE DESIGN ON PERFORMANCE}

70. METAL. - The composition of the metal has an entirely negligible effect on the heat dissipation of a radiator core, except in indirect cooling surface, for which high thermal conductivity is important. The thickness of metal is also of negligible importance for direct-cooling surface, except as it adds to the weight and as proper thickness is necessary for mechanical strength. For indirect cooling surface thickness of metal is desirable for heat transfer but undesirable because of weight. The composition and thickness of the metal must be governed very largely by considerations not taken up in this report, such as mechanical strength, durability, and ease and cost of construction and repair. Such thermal considerations as do enter (indirect surface) are discussed in articles $67-69$ and 75 .

7 I. WEIGHT. - Weight of the core should evidently be the minimum that is consistent with such governing conditions as thickness of metal and thickness of water streams. In comparison with head resistance it is of rather great importance at low speeds but little importance at high speeds (see art. 44). 
72. FREE AREA.-For unobstructed positions of a radiator a large free area is desirable in order to increase air flow and decrease head resistance and should be sacrificed only for the sake of compactness of the radiator. Care taken in making the water tubes as narrow as is consistent with sufficient strength and space for ample water flow is repaid by an increase in figure of merit. For use in obstructed positions, where head resistance of the core is relatively unimportant, high free area can well be sacrificed (see arts. 23, 4I).

73. Amount of Coolrng Surface.-A large amount of cooling surface per unit frontal area is desirable for high heat dissipation at a given air flow, but can be obtained only at the expense of reduction in free area and air flow and increase in head resistance. The heat dissipated per unit frontal area is not in general proportional to the amount of cooling surface because of (I) smaller temperature difference between air and water at the rear face than at the front, (2) smaller temperature difference between air and metal for indirect than for direct cooling surface, and (3) changes in turbulence with change in construction. Indirect cooling surface can often be used to good advantage on account of its saving in weight and its relatively small reduction of free area, but attention must be given to thermal conductivity, thermal contact with the water tube walls, and proper proportions of fins (see arts. 67-69, and art. 75).

74. Condition of Cooling Surface.-Cooling surfaces in radiators should be kept clean. An accumulation of oil and dust on the surface will have a very harmful effect on the performance of the radiator. The following remarks apply only to conditions in which the cooling surfaces are reasonably clean:

(a) Heat dissipation from an ordinarily smooth surface to a high-speed air stream may be increased $\mathrm{I} 7$ per cent for a given air flow by giving the surface extremely high polish, or it may be decreased ro per cent or more by roughening the surface with a layer of smoke deposit.

(b) Surfaces likely to be obtained in radiators, if fairly clean, will not differ in smoothness enough to give appreciable difference in heat dissipation with a given flow of air through the core.

(c) Heat dissipation from a radiator at a given airplane speed may be slightly increased if special attention be given to smoothness of surface, because this results in a small increase in the air flow through the core.

(d) Heat dissipation to a high-speed air stream is practically unaffected by a light coating of clean oil on a smooth surface. 
(e) Pressure gradient in the air flowing through a radiator tube of simple geometrical form is practically independent of the roughness of the surface over a considerable range.

$(f)$ Pressure gradient in such a tube is practically unaffected by a light coating of clean oil on a smooth surface.

(g) Head resistance of a radiator may be decreased slightly by polishing the surfaces ( 8 per cent observed in one case).

(h) Figure of merit of a radiator may be somewhat increased by polishing the surfaces.

(i) In general, the performance of a radiator may be improved a little by polishing the surfaces, but if they are fairly smooth and clean a considerable polish is required to produce much change in the properties of the radiator, and there is a question whether or not such a method for improvement be practicable.

75. Proportions of Fins for Given EFfectiveness.-Since the effectiveness of indirect cooling surface is determined by the function $u$, developed in article 68, equation (2) of that article may be solved for $y$, giving

$$
y=\frac{2 b^{2} q}{u^{2} k},
$$

which shows that the thickness of fin resulting in any given effectiveness is directly proportional to the square of the width, to the coefficient $q$, which varies approximatley as the 0.8 power of the air flow, and inversely proportional to the conductivity of the metal. If the fin is formed by folding the metal over, the thickness of fin includes both thicknesses of metal. Table 16 shows the approximate thicknesses of fin that will give effectiveness of 90 and 95 per cent with different widths. The table is computed for a long, narrow fin of rectangular form, soldered or continuous with the water-tube walls on only one edge. If both edges are in good thermal contact with the walls, the thickness may be divided by four.

If the coefficient $q$ is not known for a certain type of radiator, it may be estimated by means of equations (I) and (2) article 64. In order to show the magnitude of the effectiveness of ordinary indirect cooling surface, a computation has been made for a type similar to the radiators A 7, 8, 9, I3 (Figs. 57-60), assuming 50 per cent indirect cooling surface. In this case the effectiveness is found to be 94 per cent at an air flow of 4.3 pounds per second per square foot frontal area, or a flying speed of about 70 miles per hour ( $3 \mathrm{I} \mathrm{m}$ per sec), and 9I per cent at an air flow of 8 pounds, or a flying speed of 130 miles per hour ( $60 \mathrm{~m}$ per sec). 
The reduction in heat dissipation due to the fact that the indirect cooling surface is less effective than the direct is 3 and 4 per cent for these two cases.

TABLE 16.-Thickness (in Inches) of Indirect Radiator Fins of Brass or Copper for Different Widths and Air Speeds to Give Effectiveness of 90 Per Cent and of 95 Per Cent

[For thermal contact along one edge only. If both edges of fin make good thermal contact with source of heat, divide thickness by 4 ]

\begin{tabular}{|c|c|c|c|c|c|c|c|c|}
\hline \multirow{3}{*}{$\begin{array}{l}\text { Width } \\
\text { of fin } \\
\text { (inches) }\end{array}$} & \multicolumn{4}{|c|}{ Copper fins } & \multicolumn{4}{|c|}{ Brass fins } \\
\hline & \multicolumn{2}{|c|}{$\begin{array}{l}\text { Thickness to give ef- } \\
\text { fectiveness of } 90 \text { per } \\
\text { cent at air speeds of }\end{array}$} & \multicolumn{2}{|c|}{$\begin{array}{l}\text { Thickness to give ei- } \\
\text { fectiveness of } 95 \text { per } \\
\text { cent at air speeds of- }\end{array}$} & \multicolumn{2}{|c|}{$\begin{array}{l}\text { Thickness to give ef- } \\
\text { fectiveness of } 90 \text { per } \\
\text { cent at air speeds of - }\end{array}$} & \multicolumn{2}{|c|}{$\begin{array}{l}\text { Thickness to give } \\
\text { effectiveness of } 95 \\
\text { per cent at air } \\
\text { Speeds of- }\end{array}$} \\
\hline & $\begin{array}{c}150 \\
\mathrm{mi} . / \mathrm{hr} .\end{array}$ & $\begin{array}{c}60 \\
\text { mi./hr. }\end{array}$ & $\begin{array}{c}150 \\
\text { mi. } / \mathrm{hr}\end{array}$ & $\begin{array}{c}60 \\
\mathrm{mi} . / \mathrm{hr} .\end{array}$ & $\begin{array}{c}150 \\
\text { mi. } / \mathrm{hr} \text {. }\end{array}$ & $\begin{array}{c}60 \\
\mathrm{mi} . / \mathrm{hr} .\end{array}$ & $\begin{array}{c}150 \\
\text { mi./hr. }\end{array}$ & $\begin{array}{c}60 \\
\mathrm{mi} . / \mathrm{hr}\end{array}$ \\
\hline 0.250 & 0.0068 & 0.0032 & 0.015 & 0.0072 & 0.025 & 0.012 & 0.052 & 0.026 \\
\hline .375 & .016 & .0076 & .034 & .016 & .056 & .026 & .12 & .056 \\
\hline .500 & .028 & .013 & .060 & .029 & .100 & .048 & .21 & .104 \\
\hline 1.000 & .112 & .052 & .24 & .12 & .40 & .19 & .85 & .41 \\
\hline
\end{tabular}

76. DEPTH OF RAdiatoR.-Increasing the depth (dimension parallel to the direction of air flow) of a particular type of radiator increases its heat transfer for a given air flow by increasing the cooling surface, but the added surface becomes less effective as the depth is increased on account of the rise in temperature of the air in its passage through the radiator and the consequent decrease in temperature difference between the air and the water. Furthermore, resistance to air flow increases with increase in depth, so that the air flow at a given flying speed is less. An increase in depth is accompanied by an increase in head resistance, weight, and power absorbed, but at the smaller depths the disadvantage of the increased absorption of power is less than the advantage of the increased heat dissipation, and the figure of merit rises to a maximum at a certain depth which varies with the type of core and with the speed of flight.

In order to show the effect of depth on performance, estimates have been made for various general types of radiators by means of the empirical equations as a medium for averaging the experimental data obtained from measuring individual radiator cores, differing slightly but of one general type in each case. The average performance curves for a number of types are shown in Figs. 3 I to 34. Air flow was estimated by using empirical equa- 

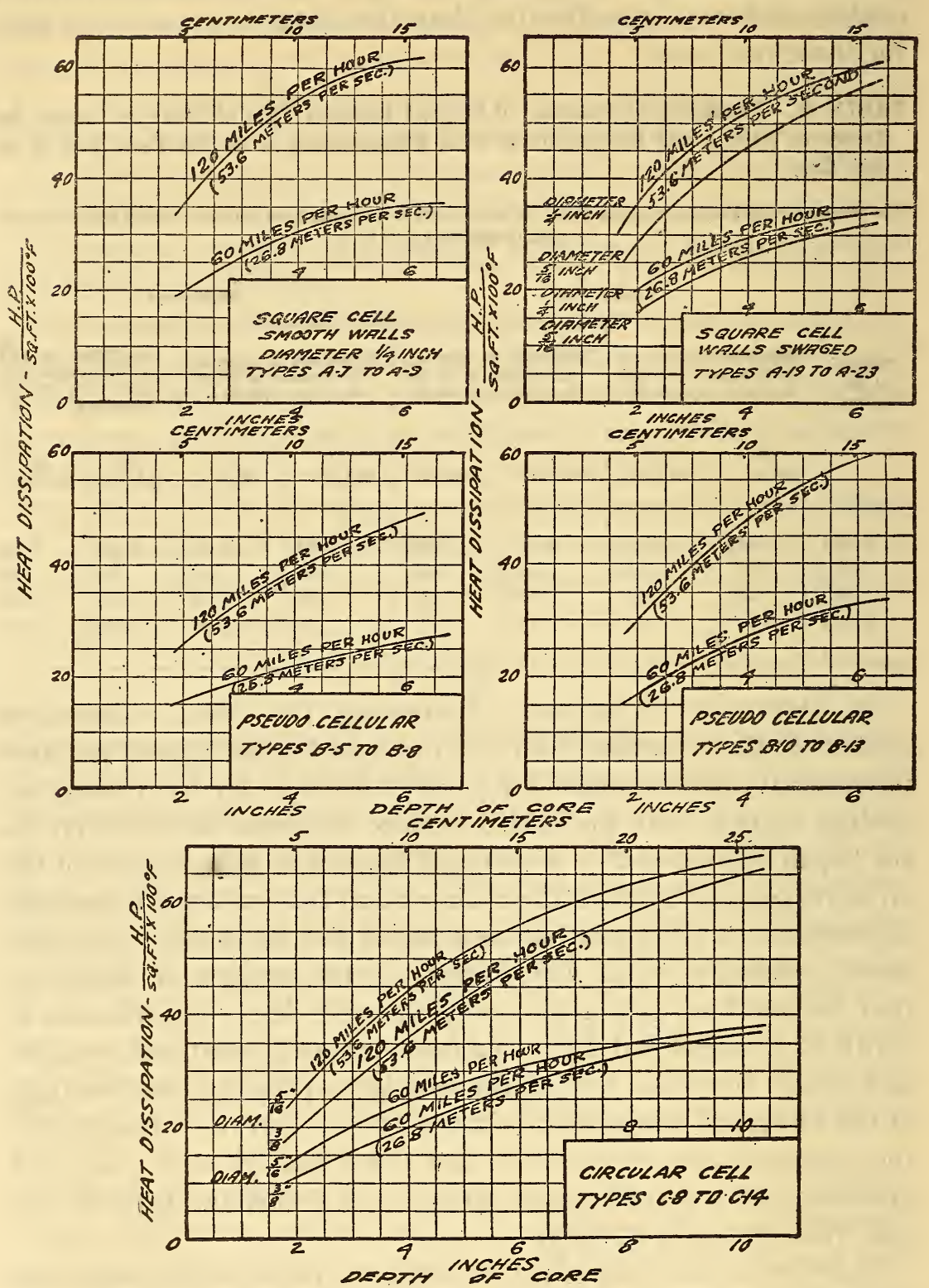

Fig. 3I.-Relation between depth of radiator core and heat dissipation, other conditions being constant

The deeper the core, the less does an additional inch of depth add to its heat dissipating ability, as indicated by the flattening of the curves toward the top. (This characteristic is, however, little in evidence for usual depth of a core with very low heat dissipation for given flying speed, such as B-5 to B-8) 
tion (I) of article 20 , and heat dissipation by using equation (7) of article 63 , taking in each case mean values of empirical constants computed from laboratory measurements with radiator cores of the type considered and mean values of geometrical characteristics for each different group.
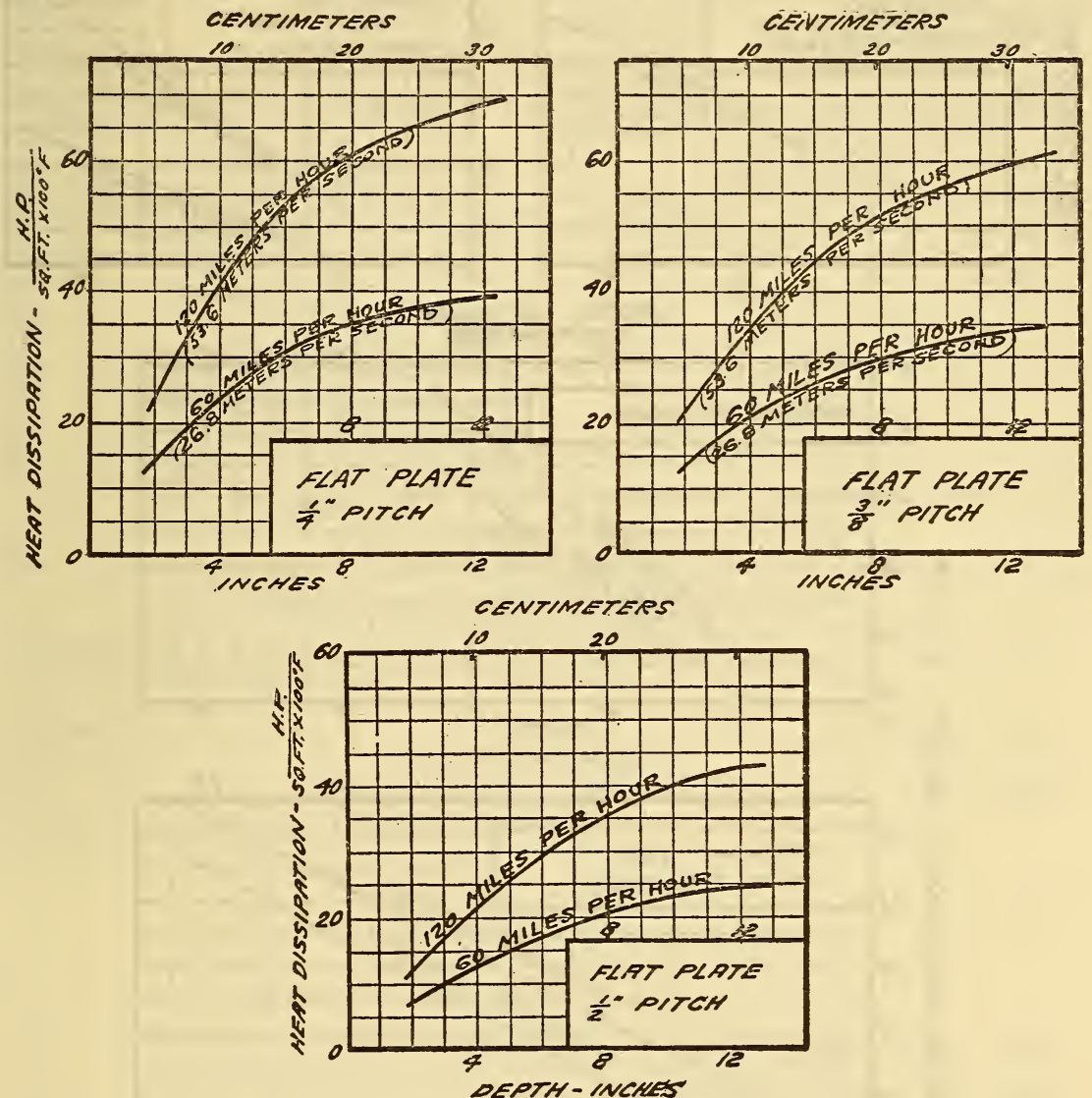

Fig. 32.-Relation between depth of radiator core and heat dissipation for flat plate cores

Curves show general trend exactly similar to those for cellular radiators plotted in Fig. 3 I

In applying equation (7) of article 63 , namely,

$$
H=M C_{\mathrm{p}} T\left(\mathrm{I}-e^{-\alpha x_{1}}\right)
$$

to this computation the following points require consideration. As noted in article 63 , the factor $\alpha$, defined by the relation

$$
\alpha=\frac{q p}{M C_{\mathrm{p}}} \quad \text { (equation } 4 \text {, art. 63) }
$$


is not a constant even for a particular type of radiator because of the variation of the quantity $q$ with changes in air flow. To reduce the labor of computing, it is very desirable to have a con-
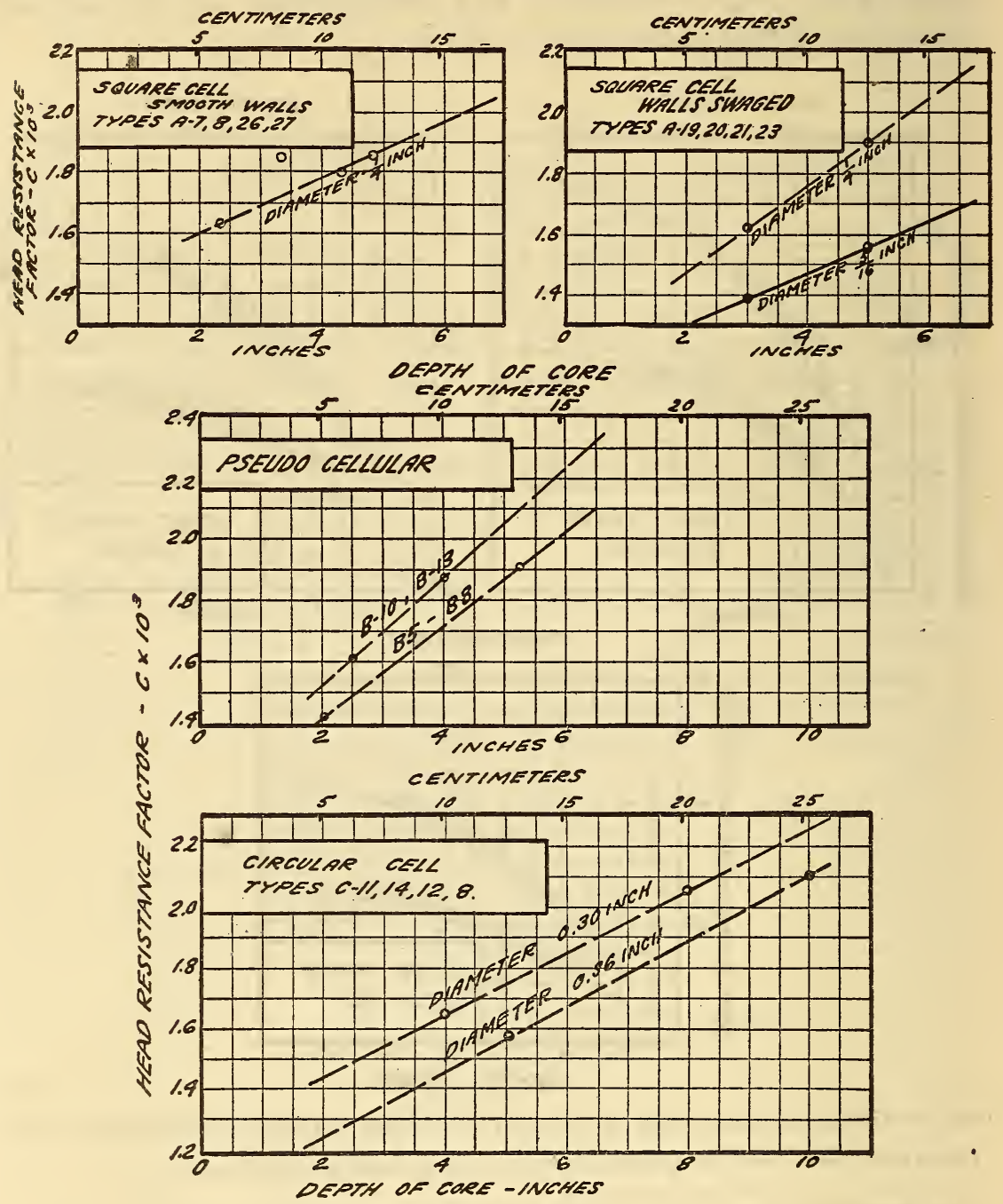

FIG. 33.-Effect of depth of radiator core on head resistance at any given speed

Head resistance factor is the head resistance per unit frontal area (lbs. per square foot for above curves) divided by the square of the flying speed (miles per hour) and is therefore nearly independent of speed. The curves indicate a linear relation between this factor and depth of radiator core. Since heat dissipation does not increase so rapidly as core depth (Figs. $3^{I-32}$ ) some optimum depth may be found for each set of conditions. See Figs. $35^{-36}$

stant for the particular core in the exponent of $e$ in the equation written above, and this is secured by introducing the empirical relation (art. 62 and Table $2 \mathrm{I}$ ), that when all other conditions are 
constant the heat dissipation of such cores varies approximately as the 0.8 power of the air flow, and $q$ is proportional to the heat dissipation.

Accordingly, for the purpose of these computations, it was assumed that $q$ varies in that manner except in the case of the flat plate radiators, for which the exponent was separately determined from the tests on each section. Thus, $q$ varies as $M^{0.8}$ and $\alpha$ varies as $M^{0.8} / M$ or as $M^{-0.2}$. Combining all the constants of
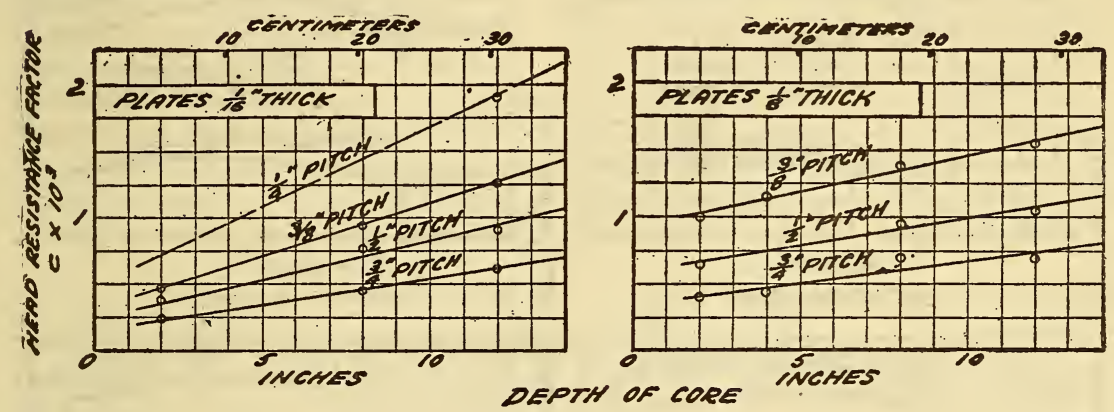

FIG. 34.-Laboratory measurements of head resistance factor for flat plate radiator cores of several depths, pitches, and plate thicknesses

The results show that over the range of depths measured, increase in head resistance is strictly proportional to increase in depth of core

proportionality into a single constant $\beta$, the computation for a given core with everything constant except air flow and depth reduces to tabulation of the product

$$
M C_{\mathrm{p}} T\left(\mathrm{I}-e^{\beta-\frac{x}{M^{0.2}}}\right)
$$

for the several values of $M$ and $x$.

For estimates of head resistance at various depths of a type core from measurements made on individual cores of the type nearly alike in other characteristics, although not identical, it was assumed that for depths greater than a certain value the resistance may be regarded as the sum of two parts-one due to eddy resistances at the front and rear faces and the other to internal resistance in the core-so that an increase in head resistance caused by increasing the depth would be proportional to the increase in depth. This assumption is supported by the results of the tests on flat plate radiators of depths from 5 to $30 \mathrm{~cm}$ ( 2 to 12 inches), which indicate that the lower limit of the range over which the assumption is valid is less than $5 \mathrm{~cm}$ ( 2 inches) of depth. Results obtained with the flat plate radiators are shown 
in Fig. 34, and Fig. 33 shows the basis of estimates for other types. In computing figure of merit the weight was assumed to increase uniformly with increase in depth.

The curves of Figs. 35-36 show (I) that the best depth for use in obstructed positions-i. e., the depth giving maximum figure
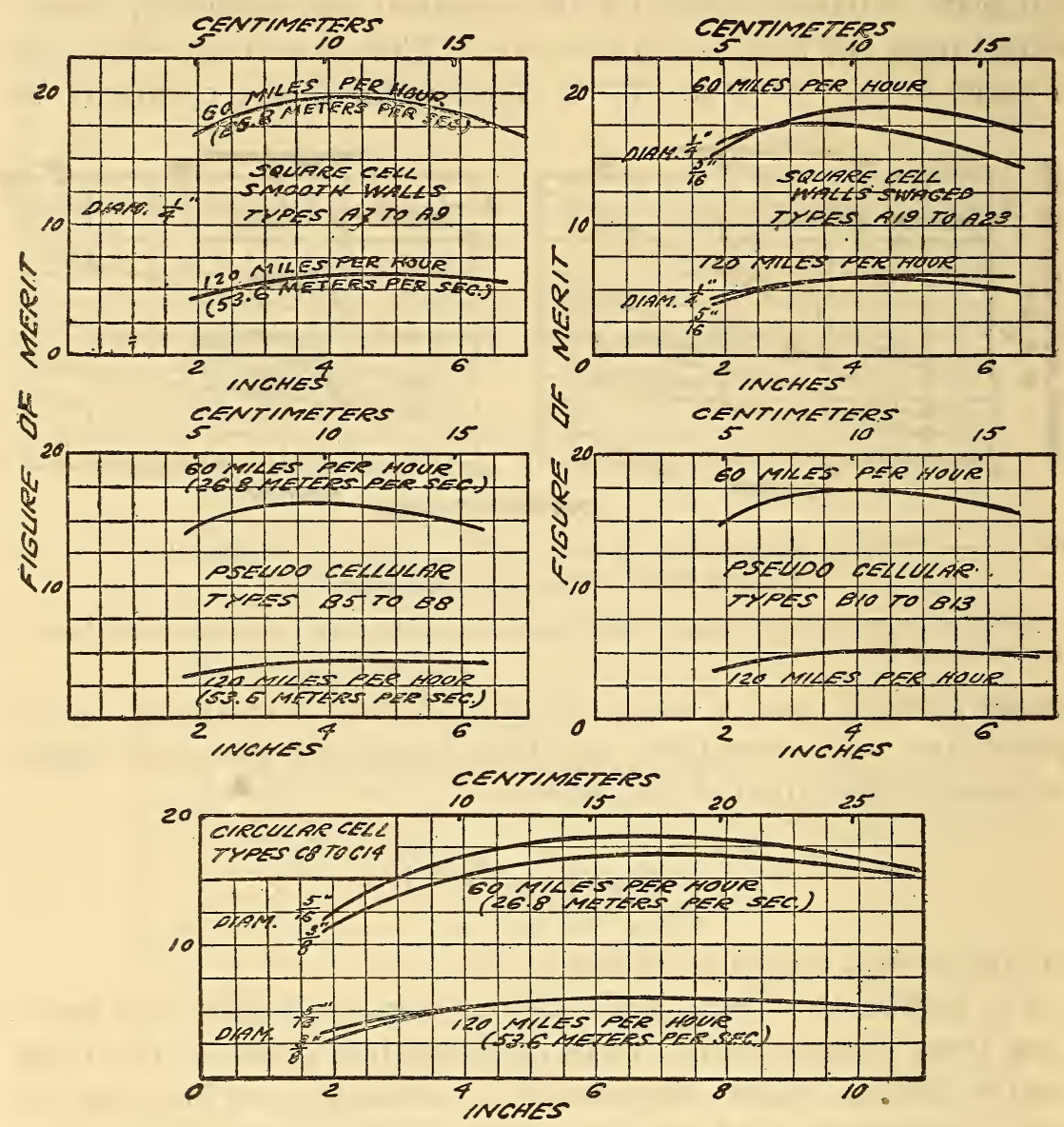

FIG. 35.-Relation between depth of radiator and figure of merit of cellular cores

Figure of merit is the ratio of power dissipated as heat to the engine power absorbed by the core in overcoming head resistance and sustaining its weight. It is therefore a measue of relative merits of radiator cores in unobstructed positions. The curves show that there is an optimum depth for given flying speed

of merit-is greater for high speeds than for low, and (2) that a small excess of depth over the optimum causes slightly less decrease in figure of merit than does an equal deficit of depth, so that an error in design on the side of too great depth is perhaps to be preferred to one on the side of too small depth. Figures for most efficient depths for the various types are collected in Table $\mathbf{7}$. 
TABLE 17.-Most Efficient Depth of Radiator Core for Use in Unobstructed Position

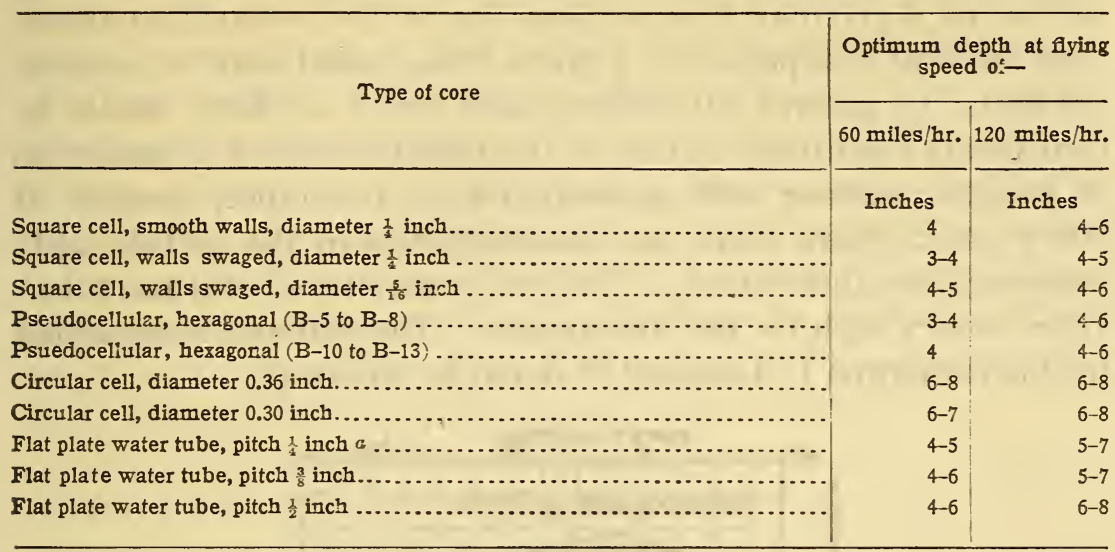

a Measured across front, center to center of plates.

77. Form and Dimensions of Water Passages.-Conditions for minimum absorption of power in pumping water through the radiator would evidently require that the water passages should be straight, with smooth walls and large cross sectional area; but the power used in driving the pump is a very small part of the engine power, and the best conditions for the pumping may well be sacrificed for those that facilitate the best relation between heat dissipation and head resistance. The thickness of the streams of water should be as small as is consistent with sufficient flow under reasonable pressure difference and security from obstruction by particles in the water, in order that the free area may be as large as possible and the head resistance and weight of water as low as possible. Pockets in the water passages in which the water is comparatively stagnant should be avoided because their heat transfer is low, they add unduly to the weight of water, and they occupy space that might better be left open for the flow of air. Cooling surface around such pockets has been rated as indirect in this report.

78. Form and Dimensions of Air Passages.-For use in unobstructed positions it is important that the air passages should be straight and as smooth as practicable to facilitate air flow and reduce head resistance. Projections, indentations, and holes in the air-tube walls add greatly to the head resistance without causing a corresponding increase in heat transfer. The slight advantage of saving in metal gained by punching holes in the indirect cooling surfaces is more than outweighted by an increased head resistance and a change in turbulence of the air which, 
although it may increase the heat dissipation per unit cooling surface for a given air flow, reduces the air flow to such an extent that the heat dissipated for a given flying speed may be actually reduced. In general, all surfaces past which air flows should be continuous from front to rear of the radiator to give a maximum of effective surface with a minimum air resistance, because at every point where there are discontinuities in the surface eddy resistances are introduced. The head resistance of "fin and tube" types is very high for just this reason. The material summarized in this paragraph is discussed in detail in article 38 .
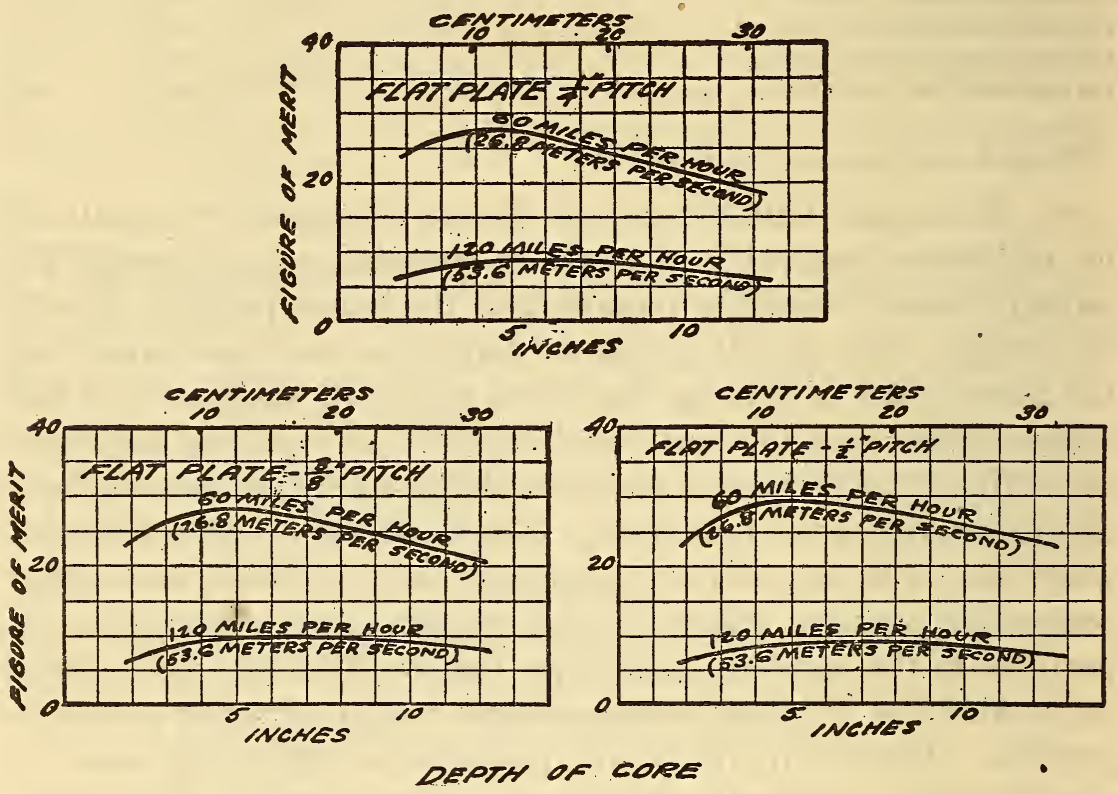

Fig. 36.-Relation between figure of merit and depth of radiator for flat plate cores

Curves show similarity to those of Fig. 35, for cellular cores. Differences in scale of abscissas account for the fact that the maxima appear to be more sharply defined than in Fig. 35

The use of spiral vanes, or other devices for increasing turbulence, although often increasing the heat transfer per unit cooling surface at a given air flow, is detrimental to the general performance of the radiator for use in unobstructed positions but advantageous in some cases for use in obstructed positions. For a comparison of square, circular, hexagonal and other types of air tubes see below, "Special types of radiators" (arts. 79 et seq.). In considering the effects of turbulence and turbulence devices on the performance of the radiator it is necessary to bear in mind the importance of the quantity of air flowing through the core and the fact that at a given speed of flight rather widely different 
amounts of air flow through different radiators. Any form of construction that imparts additional turbulence to the air may be expected to increase the resistance to flow of air and consequently to decrease the flow through the radiator for a given flying speed, while at the same time increasing the head resistance. If, then, there is to be a gain in general performance, the turbulence device must, by increasing the amount of cooling surface or by causing the air to scour the surface more thoroughly, or both, increase the heat dissipation enough to overbalance both the decrease in amount of air flow (which tends to decrease the heat dissipation) and the increase in head resistance.

The general performance of four types of radiator, each representing one of the best of its class, is shown in Fig. 37 by the "figure of merit," which is the ratio of the rate of dissipation of heat (expressed in units of power) to the power absorbed in overcoming the head resistance and sustaining the weight of the radiator. It is noticeable that at the usual flying speeds the flat plate and square cell types show much higher figure of merit than the other two types, although at a speed of $9 \mathrm{~m}$ per sec (20 miles per hour) the type with spiral vanes would probably be better than even the flat plate type. The figures of merit as drawn apply only to radiators mounted in "unobstructed" positions, such that flow of air through and around them is practically unaffected by other parts of the aircraft. For use in such positions at high speeds every form of turbulence device known to the authors is detrimental to the general performance of the radiator. On the other hand, if a radiator is to be used in such a position as the nose of the fuselage, the air flow through it at best is low and can not be increased without undue increase in head resistance of the combination of fuselage and radiator. In this case heat must be transmitted as rapidly as possible to the small amount of air that does flow through, and it may be profitable to use turbulence devices. It is possible that the rate of heat dissipation for the whole radiator may be increased, while added air resistance of the core may actually reduce the head resistance of the fuselage and radiator.

\section{B. SPECIAL TYPES OF RADIATORS}

Of the general classes into which radiators may be divided those with flat plate water tubes show the greatest figures of merit at high speeds in unobstructed positions. Comparison of different types may be made by the use of Table $2 \mathrm{I}$.

$75013^{\circ}-22-7$ 
79. Cellular Types. - The cellular types of core vary rather widely in performance, even between types with the same form of cell; but of the radiators tested the best of the square cell types are better than the others, with the separate air tube types (circular and hexagonal) ranking second. The circular cell type has

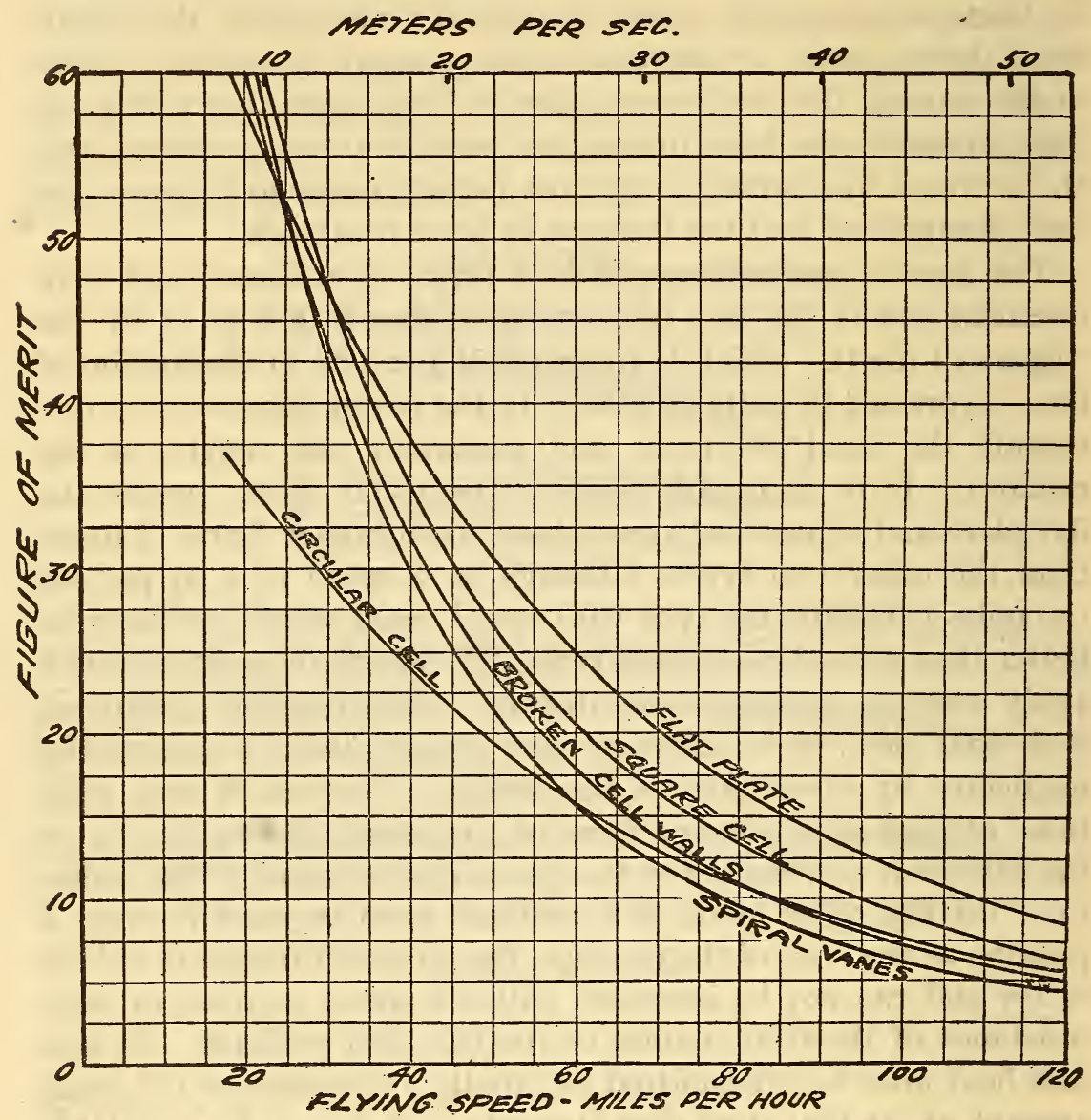

FIG. 37.-Figure of merit of typical radiator cores in unobstructed position

Ratio of power dissipated as heat to porver absorbed in overcoming head resistance and sustaining the weight. A measure of comparative merits of the several types at any given flying speed. The curves show that devices to increase turbulence in the air tubes of a radiator (spiral vanes, broken cell walls, etc.) are detrimental in unobstructed positions for all customary flying speeds

been largely used by both the American and British Governments, not because they have the highest figure of merit, but principally because of such other considerations as ease of repair.

8o. Flat Plate Types.- - Radiators whose water tubes are plain hollow plates continuous from front to rear of the core and with no indirect cooling surface show higher figure of merit at high 
speeds than any other form that has been tested. While the plates are mechanically weak, the advantages in performance are so great as to merit special study, and the types $\mathrm{E}_{-}-6, \mathrm{E}-7$, and $\mathrm{E}-8$ (Figs. IO9-III) have been used as a basis for estimation of the properties of similar types of other depths.

Computations were made as outlined in article 76 , using empirical constants computed from measurements for these three types. For head resistance a set of 22 dummy radiators was constructed using depths of $2,4,8$, and 12 inches, spacings from center to center of plate of one-fourth, three-eighths, one-half, three-fourths, and I inch, and thicknesses of one-sixteenth and one-eighth inch (depths 5.I, I0.2, I5.2, and $30.5 \mathrm{~cm}$, spacings $0.63,0.95,1.27, \mathrm{r} .9 \mathrm{r}$, and $2.54 \mathrm{~cm}$, thicknesses 0.16 and $0.32 \mathrm{~cm}$ ). The plates were made by covering cardboard of the proper thickness with thin sheet copper, and the same plates were used repeatedly. They were 12 inches long, and, with the exception of the one-fourth inch spacing, enough were used to make a radiator approximately I foot wide. The surfaces were not as smooth as might have been desired, but were fairly representative of what is found in commercially nianufactured radiators.

The measurements of head resistance are recorded in Table 30 of Appendix G. It was found that for plates one-sixteenth inch (o. $16 \mathrm{~cm}$ ) thick the head resistance factor $c$ (applicable to the equation $R=c S^{2}$ at speeds near 60 miles per hour) for the English units was well represented by the equation,

$$
c=n(1.0+2.5 x)(10-5)
$$

where $c=$ head resistance factor (for 60 miles per hour).

$n=$ number of plates per foot width of core,

$x=$ depth of core, inches.

The agreement between this equation and the observed data is indicated on the left half of Fig. 34, in which the lines represent the equation and the circled points the observations. Weight of the core was estimated from geometrical considerations, and the densities of copper and solder to be

$$
w=0.056 n x
$$

where $w=$ weight of core and contained water, pounds per square foot frontal area.

In Fig. 32 the heat dissipation and in Fig. 36 the figure of merit are shown in terms of depth of core. The curves show that the wider spacings are a little better than the narrowest, although 
there is not much difference in figure of merit between the threeeighths and one-half inch spacings. The flatness of the figure of merit curves at the higher speeds shows that if considerations of compactness make it desirable to increase the depth over the optimum value this can be done without much sacrifice in figure of merit. The flat plate types are characterized by high.air flow and low head resistance for a given flying speed and by high figure of merit at the greater speeds.

8I. Fin and TUbe Types.-In general, fin and tube types are characterized by high head resistance and low air flow and partly by the large amount and of ten the low effectiveness of the indirect cooling surface. The form of construction gives large eddy resistance, because each water tube is subjected to impact on one side and suction on the other, with the result that the total (projected) area subjected to impact and suction on all the tubes is much greater than that necessitated in a cellular radiator of the same size or cooling surface.

Excessive head resistance, accompanied by low heat dissipation, makes the fin and tube types unsuitable for use on an airplane where they will be exposed to a current of air at high speed. The more compact types, however-notably F-4, which has large water tubes with crimped spiral fins nearly touching each othershow a relatively high heat dissipation at very low air flows, which justifies their use on trucks and automobiles other than racing cars.

Figs. 38 and 39 show heat dissipation of five types in terms of air flow through the core and in terms of flying speed in unobstructed positions for the types of fin and tube construction for which the air flow constant has been measured. No air flow constant was found for $\mathrm{F}-3$ because of damage to the section before the measurement was completed, and none is given for $\mathrm{F}-4$ because the air flow was too low to be measured by the air venturi meter.

The only type for which head resistance was measured is $\mathrm{F}-6$, which is similar to $\mathrm{F}-5$, except that its depth is not quite so great. In order to give some indication of what figure of merit may be expected from a fin and tube type, the head resistance and power absorbed for F-6 have been combined with the heat dissipation and air flow of $\mathrm{F}-5$ in computing the curves shown in Fig. I 5 . This, of course, gives the figure of merit slightly higher than the type $\mathrm{F}-5$ would give, but even with an error on the side of good performance the result shows that the type is not suitable for 
general aeronautic use. It is possible that for a wing position, where the air flow is low and head resistance of the core is not a detriment, a fin and tube radiator could be used.

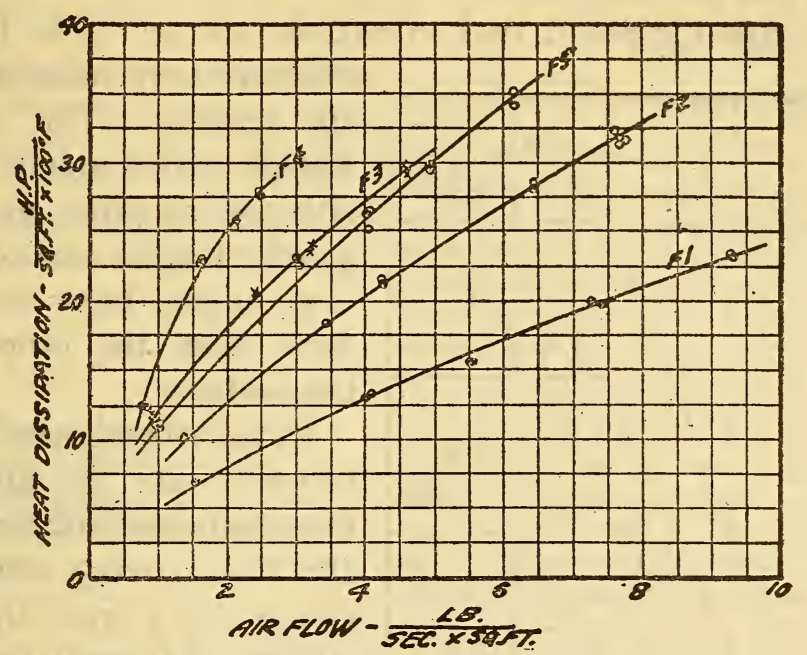

FIG. 38.- Heat dissipation of fin and tube radiators as a function of air flow through core

$\mathrm{F}-\mathrm{x}$ and $\mathrm{F}-2$ are similar except that fins on $\mathrm{F}-2$ are spaced only half as far apart as on $\mathrm{F}-\mathrm{r}$. $\mathrm{F}-3$ has fins of same spacing as $F-2$, but the water tubes are much smaller and closer, raising effectiveness of fins. $F-4$ has water tubes closely packed in comparison with the others listed above, and a relatively narrow spiral fin girdles each tube

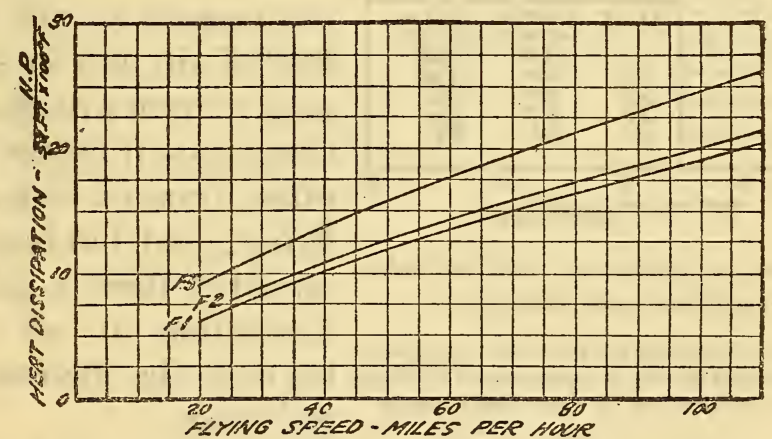

Fig. 39.-Heat dissipation of fin and tube radiator core in terms of flying speed

Comparison with Fig. 38 shows that the great difierence in heat dissipation of cores $\mathrm{F}-\mathrm{I}$ and $\mathrm{F}-2$ for the same air flow through the core makes little difference in effective heat dissipation, the reason being that the closer fin spacing of $\mathrm{F}-2$ cuts down the air flow so much more that at given flying speed the resultant effect of the two cores is nearly the same

82. WhistLING TyPes. - Three general types of construction have been found to cause the radiator to whistle in an air stream. The three classes are (I) plain water tubes separated. from each other, E-9, (2) perforated water tubes separated from each other, 
$\mathrm{E}-\mathrm{I}$ to $\mathrm{E}-5$, and (3) corrugated plates, with air flowing across the corrugations, E-IO.

The form of construction leaves passages across the air tubes in which are short columns of air over the ends of which air is blowing, with the result that vibrations are set up in the short

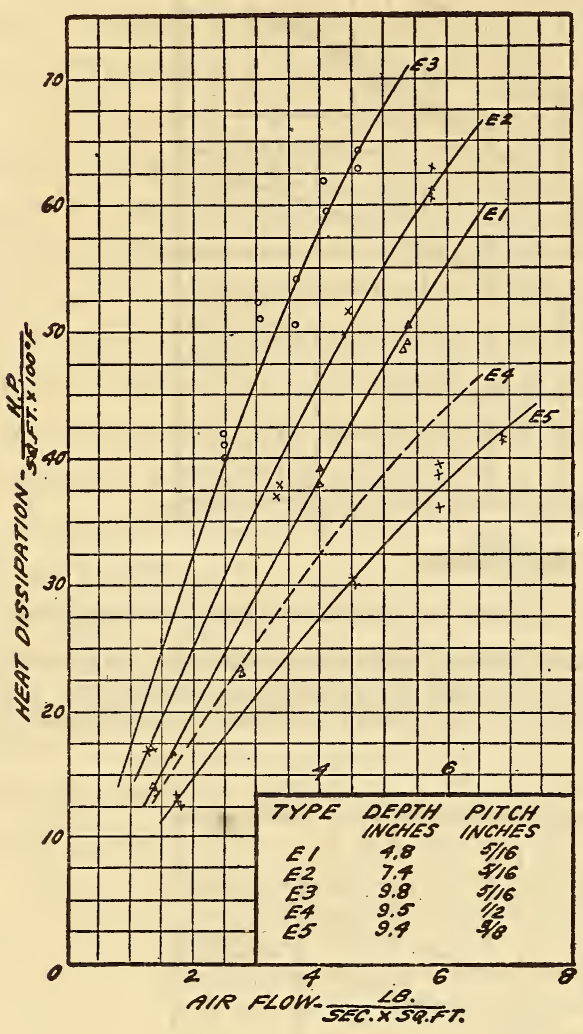

FIG. 40.-Heat dissipation of whistling radia. tors-perforated water tube type

Although heat dissipation for given air flow is high, the type is not good for use in unobstructed positions because of very low air flow for given flying speeds columns perpendicular to the air streams. The resulting whistle varies widely in intensity and in pitch as the air speed changes, and conditions of resonance have marked effects upon the properties of the radiator.

By the vibrations of the cross columns air is alternately forced into and withdrawn from the fast moving stream and causes a very great drag on the stream. As a result the air flow for a given flying speed is very low and the head resistance is very high. The very great turbulence in the air flow tends to compensate for the decreased flow of air, and in some cases may prevent a decrease in heat dissipation from the value for other forms of core at a given flying speed, but in no case observed is there a gain in heat dissipation at all comparable with the increase in head resistance.

The heat dissipation in terms of air flow is shown for the types $\mathrm{E}-\mathrm{I}$ to $\mathrm{E}-5$ in Fig. 40, the curve for $\mathrm{E}-4$ being obtained by interpolation ${ }^{26}$ between $\mathrm{E}-3$ and $\mathrm{E}-5$. The complete properties of the other types are shown in the series of general plots of properties (Part VII, art. IO9). The superiority of the flat plate (continuous from front to rear of core) type over the whistling types is indicated by the comparisons made in Table $\mathrm{I} 8$.

\footnotetext{
$2 \epsilon$ In the types $\mathrm{E}_{-3}-3$ and $\mathrm{E}_{-}-5$, which are of the same depth as $\mathrm{E}-4$, but with different pitch, the heat transfer was found proportional to the number of plates per unit width of core, and this proportionality was used for interpolation.
} 
TABLE 18.-Comparison of Flat Plate and Perforated Plate Radiators

\begin{tabular}{|c|c|c|c|c|}
\hline Type & Characteristics & Depth & Pitch & $\begin{array}{l}\text { Cooling sur- } \\
\text { face per unit } \\
\text { frontal area of } \\
\text { core }\end{array}$ \\
\hline E-4... & Perforated plate (whistling).............. & $\begin{array}{r}\text { Inches } \\
9.5\end{array}$ & $\begin{array}{l}\text { Inches } \\
0.5\end{array}$ & Ratio a \\
\hline $\mathbf{E}-8 . \ldots$ & 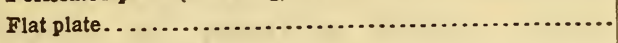 & 9.75 & .5 & 39 \\
\hline
\end{tabular}

a Ratio (square feet per square foot) independent of unit.

Properties at Flying Speeds of 60 and 90 Miles Per Hour (1 Square Foot Frontal Area)

\begin{tabular}{|c|c|c|c|c|}
\hline \multirow{2}{*}{ Properties } & \multicolumn{2}{|c|}{$60 \mathrm{mi} . / \mathrm{hr}$. } & \multicolumn{2}{|c|}{$90 \mathrm{mi} . / \mathrm{hr}}$. \\
\hline & E-4 & E-8 & E-4 & E-8 \\
\hline Air flow, pounds per second ............... & 2.63 & 3. 79 & 3.94 & 5.68 \\
\hline Heat dissipation, horsepower per $100^{\circ} \mathrm{F} .$. & 23.3 & 22.3 & 32.3 & 30.6 \\
\hline Head resistance, pounds......................... & 6.7 & 3.0 & 15.0 & 6.6 \\
\hline Power absorbed, horsepower .......... & 1.75 & .89 & 4.62 & 2.20 \\
\hline Figure of merit....................... & 13.3 & 25.0 & 7.0 & 13.9 \\
\hline
\end{tabular}

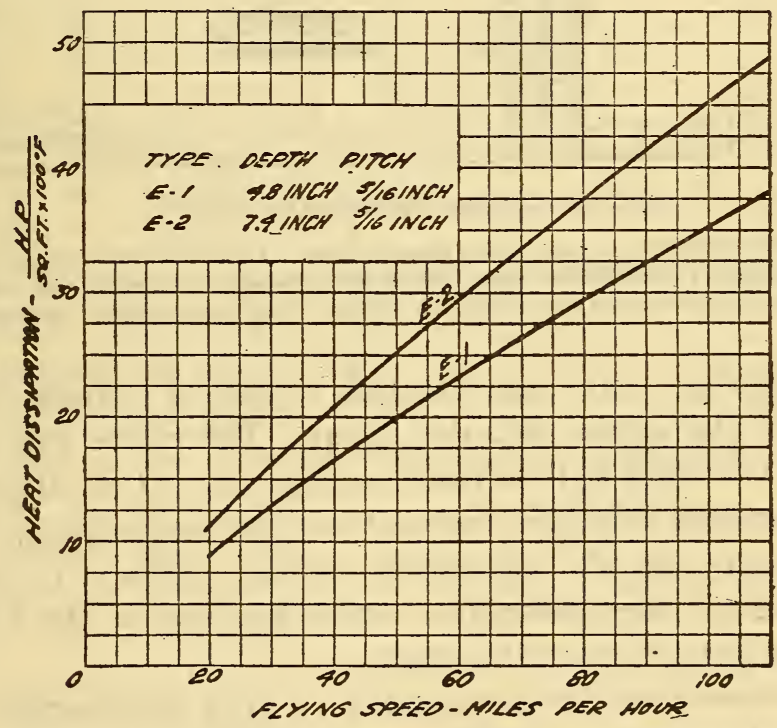

FIG. 4I.-Heat dissipation of whistling radiators (perforated tube type) in terms of flying speed

83. Wing AND Strut SURFAcE.-Various devices have been suggested, such as using the underside of the wing for the cooling surface or building the struts and other parts of the airplane with double walls and passing water through the spaces formed. While such construction might furnish heat transfer with no great increase in head resistance, it involves mechanical difficulties in 
construction. It is essentially heavy because of the two walls necessary to confine the water streams; only one is available for effective cooling surface. Furthermore, to keep frictional resistance (water) within reasonable limits the water streams must be fairly thick on account of their great length. The construction would obviously be unsuitable for military use because of its vulnerability. Such surface would probably be slightly less effective in dissipating heat than would an equal area of cooling surface made up into a good form of radiator.

84. Stream-Line CaSing FOR RAdiator.-It has been suggested that a stream-line casing around a radiator might improve its performance by reducing head resistance, and a limited study has been made of the effect of such a casing. The radiator used

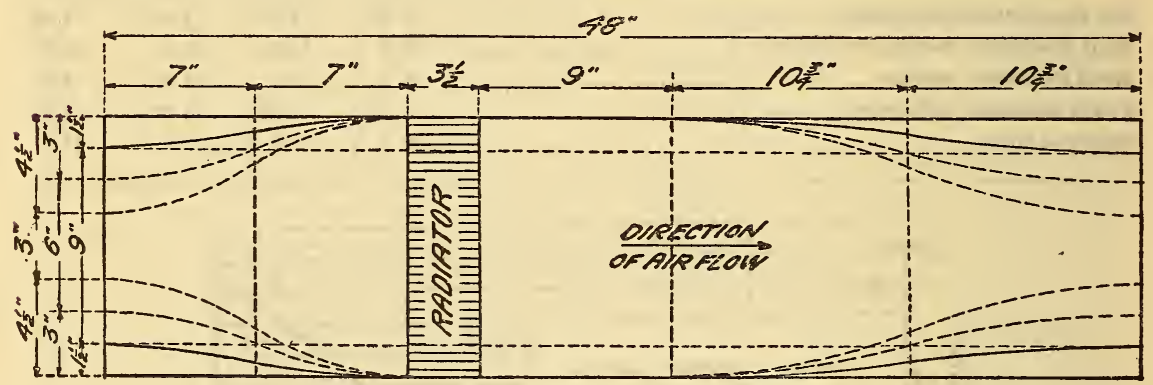

FIG. 42.-Stream-line casing for radiator

The sides of casing are curved, top and bottom of casing plane. The curved sides were set in each of the three positions shown. The stream-line casing reduces head resistance very greatly and reduces air flow through the radiator, and therefore heat dissipation. The net result under different conditions is shown in Fig. 44

was a I 2-inch $(30 \mathrm{~cm})$ square section of core of $1 / 4$ inch $(0.63 \mathrm{~cm})$ square cells $33 / 8$ inches $(8.6 \mathrm{~cm})$ deep. The casing was made of galvanized iron and is illustrated in Fig. 42 . It is stream lined in one dimension only, the top and bottom pieces being flat rectangular sheets and the side pieces curved. Arcs of circles were used instead of the stream-line curves because of the fact that air flows on both sides of the sheet.

Fig. 43 shows that the casing causes a very considerable reduction in head resistance. With certain widths of mouth it also greatly reduces the air flow through the core. As the side pieces are brought nearer together and the area of the mouth reduced, the head resistance first decreases to a minimum and then increases. The lowest resistance found was about 50 per cent of that of the unstreamlined core, with a mouth area of one-half of the frontal area of the core. Air flow through the core was measured by pitot static tubes, one at the rear mouth of the casing and the other 
at the front mouth (this latter tube was supported behind the casing, extending through the casing and core to the front mouth). The air flow obtained is represented in Table I 9 by the values of $k_{\mathrm{s}}$ for the equation

$$
M_{\mathrm{s}}=k_{\mathrm{s}} S
$$

where $\quad M_{\mathrm{s}}=$ air flow in pounds per second per square foot of core. $S=$ wind tunnel air speed, miles per hour.

(Air density $=0.0750$ pounds per cubic foot.)

TABLE 19.-Air Flow in Stream-Lined Radiator

\begin{tabular}{l} 
Mouth opening \\
\hline
\end{tabular}

By means of the head resistance, the weight and the heat transfer corresponding to the air flow, figure of merit was computed for each width of mouth and is shown in Fig. 44. In order to show the effect of weight, it has been computed for casings of galvanized iron and of aluminum. The curves show that at low speeds the increase in weight and decrease in air flow are so great as to be detrimental to the general performance. But at high speeds weight is of much less importance in comparison with head resistance, and

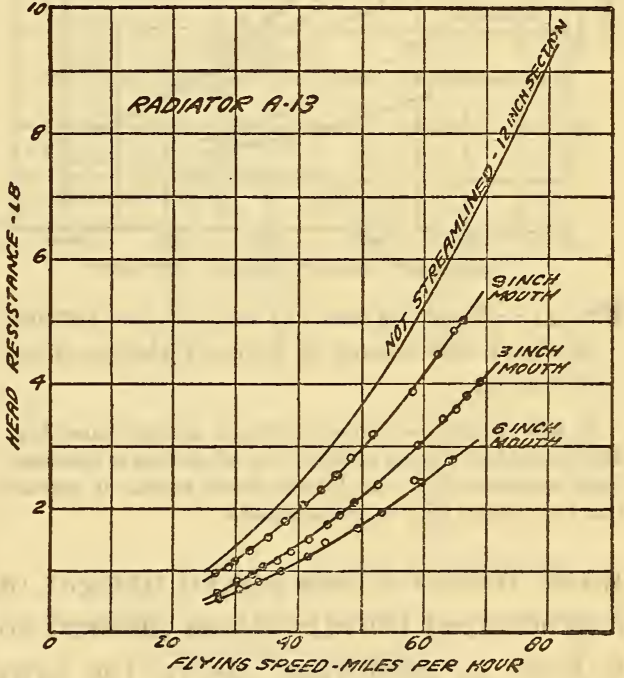

FIG. 43.-Head resistance of stream-line casing inclosing radiator core

The core section was $I_{2}$ inches square, and a casing of the form shown in Fig. 42, with 6-inch mouth, reduces the head resistance of the combination by one-half. Neither the smaller mouth area ( 3 inch) or larger mouth area ( 9 inch) is nearly so effective in this respect

the net effect of the casing seems to be a gain in performance. An easy method of shuttering could be provided by making the stream-line sides of the casing adjustable for different widths of mouth. 


\section{METHODS OF MEASURING GEOMETRICAL CHARACTERISTICS}

The following characteristics of the core were measured:

(a) Dimensions of the water tubes: Length (in the direction of water flow), depth (parallel to the direction of air flow), thickness

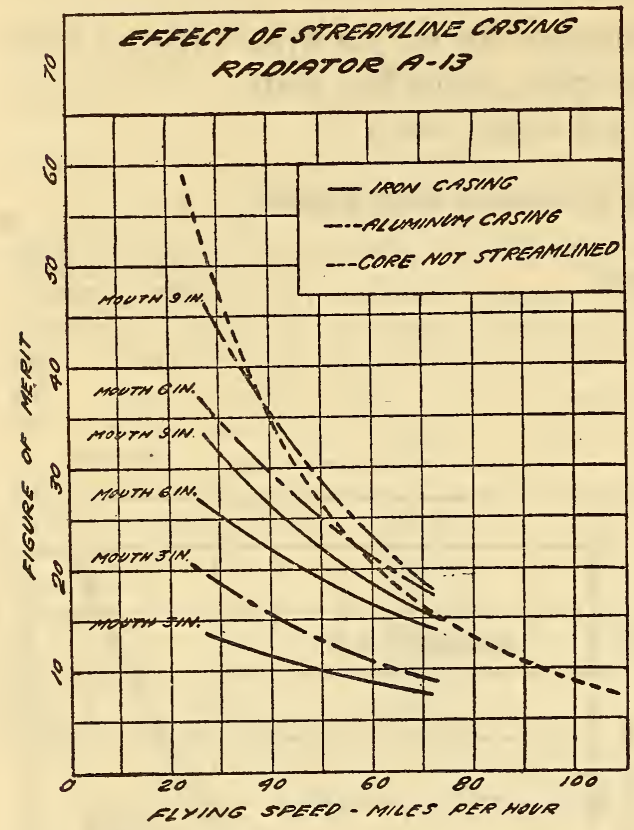

FIG. 44.-Figure of merit of radiator core inclosed in stream-line casings of different shapes, shown in Fig. 42

At low speeds, the increased weight and decreased heat dissipation fail of being offset by the advantage of decreased head resistance. At the higher flying speeds, it appears that such design may be advantageous (perpendicular to length and depth), and hydraulic radius (quotient of cross section by perimeter).

(b) Dimensions of air tubes: Depth (parallel to air flow) and hydraulic radius.

(c) Free area of air passages.

(d) Extent and distribution of cooling surface.

(e) Weight of core, empty and filled with water.

(f) Nature and thickness of metal.

(g) Shape of air and water passages.

85. Measuring DimenSIONS OF THE WATER TuBEs. - The apparatus used to measure the cross section of the water tubes is shown in Fig. 45. A radiator section $R$, with water tubes $t-t$, was placed upright on a support and water from a graduated burette $B$ was allowed to flow into it until it reached a level $h_{2}$ somewhat above the lower ends of the water tubes. This level $h_{2}$ was read on the gage $G$ and the position of the water surface on the burette scale was also read. More water was admitted and readings taken at from 6 to ro levels up to one such, as $h_{1}$, near the upper ends of the water tubes. The burette was then lowered to the position (I), and similar readings were taken while emptying the radiator. The burette was calibrated by weighing its contents at various levels, and the gage was calibrated by comparison with a dividing engine. Readings were taken to $\mathrm{I} \mathrm{cm}^{3}$ in the burette and to $0.0 \mathrm{I} \mathrm{cm}$ in the gage. 
Volumes in $\mathrm{cm}^{3}$ were plotted against heights in $\mathrm{cm}$, and since all of the water that flows out of the burette passes into the radiator and the gage the slope of the resulting line (in $\mathrm{cm}^{3}$ per $\mathrm{cm}$ ) is the area of cross section of the water tubes and the gage. The area of the gage was determined by noting the heights occupied by known volumes of water. Two or more tests were made on each section, both while filling and while emptying it.

The number of tubes was counted and their depth measured with a rule and calipers. In cases where the tubes were not vertical, as in hexagonal cell types, the horizontal area computed as explained above was projected on a plane normal to the direction of water flow. From the total cross sectional area of the water tubes, their number, and their depth, the thickness and hydraulic radius were computed.

86. MEASURING DiMENSIONS OF THE AIR TUBES. - The method of measuring the air passages was similar to

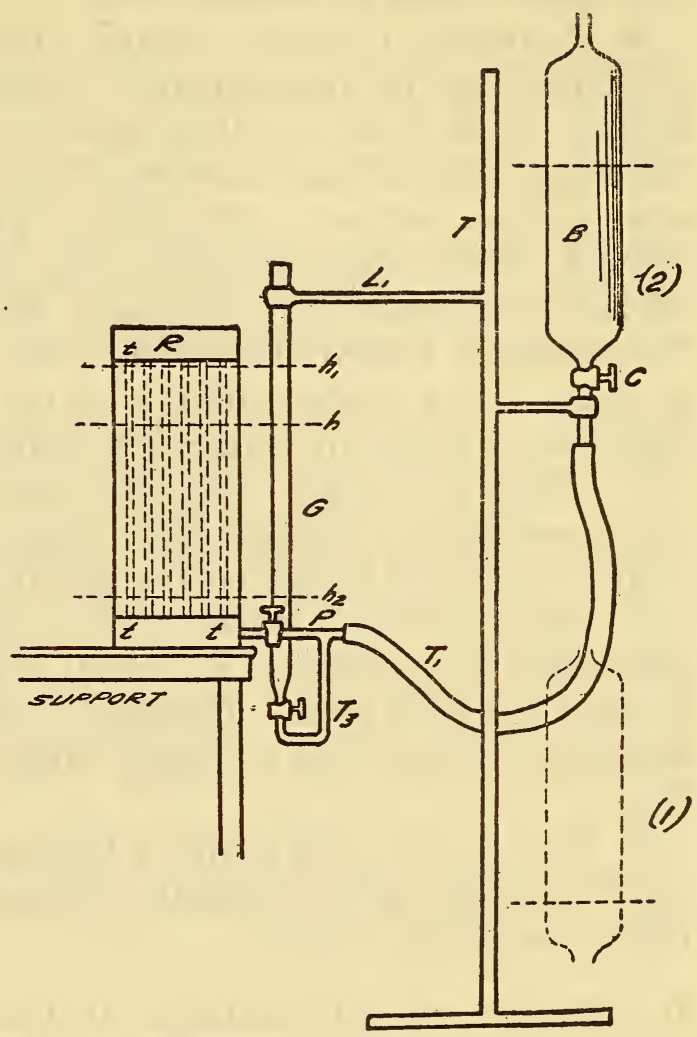

Fig. 45.-Apparatus for measuring cross sectional area of water tubes in a radiator

The principle is to measure by a burette $(B)$ the volume of water filling a selected length of a radiator tube cluster, measured on the that used for the water gage $G$

tubes. The section was clamped face down on the top rim of a shallow tank about $18 \mathrm{~cm}$ ( 7 inches) square, provided with two openings to which rubber tubes were attached, leading to the burette and the gage. The rim of the tank was $2.5 \mathrm{~cm}$ (I inch) wide and faced with a strip of soft rubber, so that the edges of the section could be sealed water-tight. The perimeter of the air tubes was determined from micrometer measurements on the 
inside of the tubes, which also furnished a check on the measurement of their area. Depth of the air tubes is identical with that of the core and was measured with a rule and calipers.

87. Computing FrEE AREA.-The free area of the air passages is the ratio of the area of the air passages to the frontal area of the core, due attention being given to the number of tubes filled with water during the measurement.

88. Computing Cooling Surface.-Cooling surface, in the case of simple types of construction, is evidently the product of the number of air tubes by their perimeter and by their length. Inspection showed what fraction of the surface was direct and what fraction indirect. The ends of the tubes were rated as indirect. When the construction was irregular, many different methods of measurement were used, depending upon the form. Two observers worked independently, and every effort was made to obtain values reliable within 2 per cent, but in few cases they could not be fixed with certainty to better than ro per cent.

89. WEIGHT OF CORE.-This was determined by weighing the empty core, without headers, on a balance. The computed water content was added to this for the weight when filled.

9o. Composition of Metal.-No attempt was made to determine exact composition of the metal, which has been described as either copper or brass. Thickness of metal was measured with micrometer calipers and is reliable within about 0.001 inch (0.02 $\mathrm{mm})$.

91. Form of Air and Water Passages.-Was determined by inspection aided by approximate measurements and is shown in Table 20 and Figs. 5 I-I 2 I.

\section{EFFECTS OF CONDITIONS OF USE ON PERFORIMANCE}

\section{A. POSITION}

This section, like the preceding chapter, is partly in the nature of a summary and will involve a certain amount of repetition of previous statements.

92. UnOBSTRUCTED Positions.-Unobstructed positions have the following advantages:

(a) Except for effects of the slip stream the air flow through the core, and consequently the heat dissipation, are greater for unobstructed than for obstructed positions.

(b) Head resistance chargeable to the radiator is considerably less for unobstructed positions than for positions in the nose of the 
fuselage, inside of the fuselage, and probably for positions in the wing. The effect of the slip stream is to increase the head resistance of a radiator that would otherwise be unobstructed.

(c) Since air flow is greater in unobstructed than in obstructed positions, and therefore heat dissipation per unit frontal area is greater, it follows that the weight of a radiator may be less for a given cooling capacity when in an unobstructed than when in an obstructed position.

(d) With reduction both in weight and in head resistance chargeable to the radiator, the power absorbed chargeable to the radiator is reduced by placing the radiator in an unobstructed position rather than in an obstructed position.

(e) With both increase in heat dissipation and decrease in power absorbed, the figure of merit of an unobstructed radiator is considerably greater than that of the same type of radiator in an obstructed position.

Some unobstructed positions have the disadvantages of requiring long connecting pipes and of being poorly adapted to shuttering the radiator. Methods of shuttering are discussed below.

93. NOSE OF THE FUSELAGE.-The location of the radiator in the nose of the fuselage is objectionable because of very large absorption of power for a given cooling capacity. Not only is the resistance of the airplane much greater with a nose radiator than with the nose properly stream lined and an unobstructed radiator of equivalent cooling capacity added, but the air flow through the core is so low that with engines of the higher powers it becomes necessary to.enlarge the fuselage to accommodate a nose radiator of size sufficient to cool the engine. Positions inside of the fuselage have been tried, but are open to the same objections as positions in the nose.

94. Wing Positions.-The performance of a radiator in the wing has not been thoroughly investigated because of experimental difficulties, but enough data are available to show that the air flow, and consequently the heat dissipation, are very low for a given flying speed, while the effect on the wing as such can only be detrimental, and the power absorbed chargeable to the radiator is probably large.

95. Slip-Stream Positions.-The effect of the propeller slip stream is to increase to some extent the air flow and heat dissipation of the radiator. No specific statement can yet be made in regard to its effects on the head resistance of radiators in obstructed positions. 
96. YAWED PosiTIONS.-The effect of yawing the radiator, or turning it so that the air strikes it at some other angle than parallel to the axis of the air tubes, is to decrease the air flow somewhat and to increase the head resistance. No experimental work has been done at this Bureau to determine the effect of yawing on heat dissipation, but both French and British reports state-that a small increase has been found with certain angles and with certain types of core. The magnitude of the effect of yawing greatly varies with different types of radiator (see art. 39).

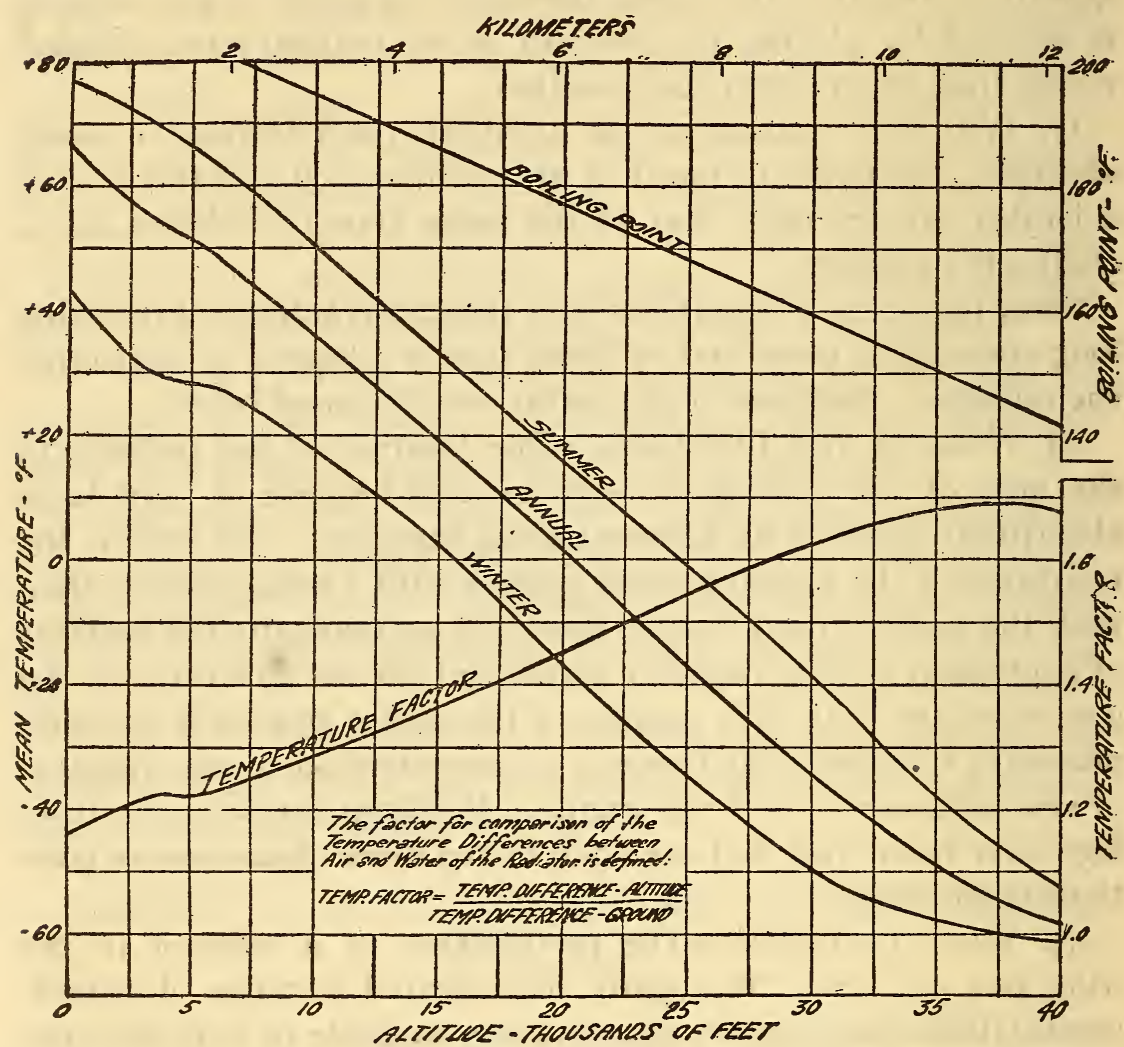

FIG. 46.-Atmospheric conditions at altitude. Temperature (mean weather for United States)

Boiling point is computed from mean pressure, the difference between summer and winter values not. affecting boiling point appreciably for this application

\section{B. EFFECTS OF ALTITUDE CONDITIONS ON PERFORMANCE}

97. Atmospheric Conditions at Altitudes.-At high altitudes both the density and the temperature of the air are less than at the ground, and changes in these conditions have important effects upon the performance of the radiator. The decrease in air 
temperature tends to increase the cooling capacity, while the decrease in density of the air tends to decrease both the cooling capacity and the absorption of power.

For a given flying speed both the air flow through the core and the head resistance are proportional to the density. Heat dissipation is practically proportional to the temperature difference between air and water, and for a given temperature difference and air flow (mass flow of air) is found to be independent of density (see art. 6I).

Since atmospheric conditions are changing continually, it is evident that any estimate of performance at an altitude must be

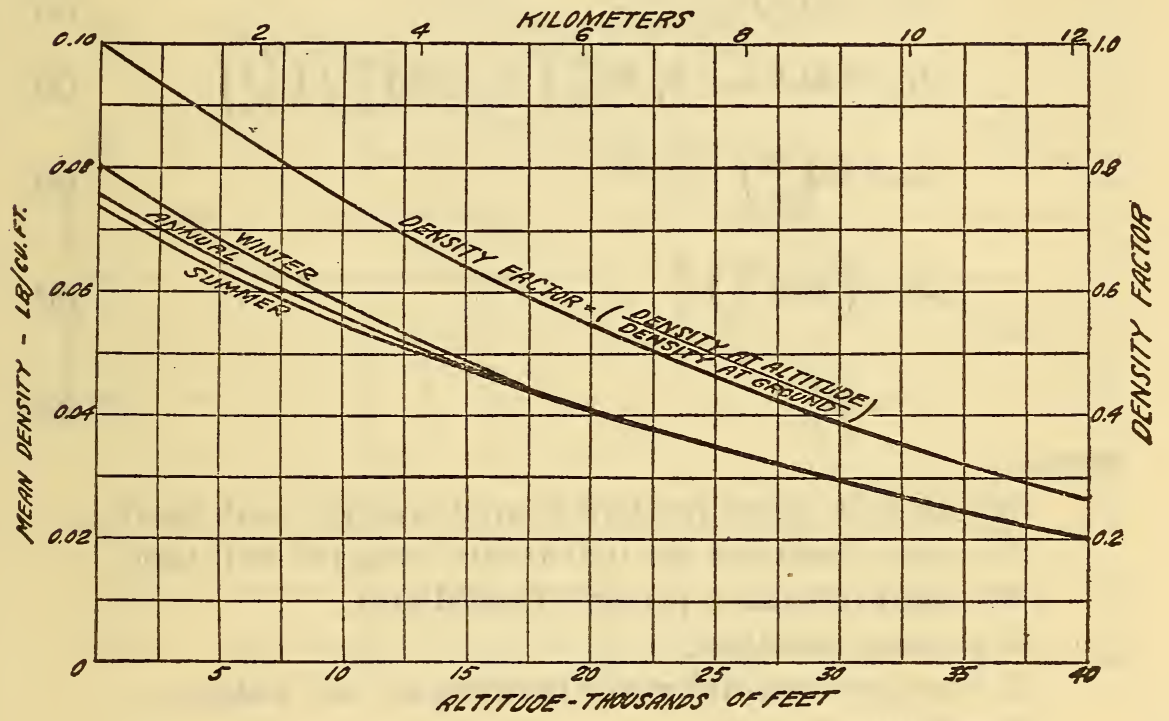

Frc. 47.-Atmospheric conditions at altitude. Density (mean weather for United States)

based upon assumed conditions, such as mean summer density and temperature, and also upon assumed requirements of operation, such as the maximum allowable water temperature. Probable atmospheric conditions at altitudes may be estimated from Figs. 46 and 47 , which are drawn from data given by W. R. Gregg in the Monthly Weather Review, January, I918, based upon observations at four stations situated in Indiana, Nebraska, South Dakota, and California. The boiling points shown correspond to mean annual pressure, only one line being plotted, because the difference between annual and summer or winter averages $\left(1.5^{\circ} \mathrm{F}\right.$ at 25,000 feet and less at lower altitudes) is so small that it may safely be neglected, in view of the uncertainties in all altitude conditions. 
98. Equations of Performance.--If the performance of a radiator at the ground is known for a particular flying speed, its performance at the same speed at altitudes may be estimated by the following equations, using the subscripts $a$ and $o$ to denote values at altitudes and at the ground, respectively, and noting that density and temperature difference "at the ground". are to be taken as the density and temperature difference to which tests at the ground are reduced:

$$
\begin{aligned}
M_{\mathrm{a}} & =M_{\mathrm{o}}\left(\frac{\rho_{\mathrm{a}}}{\rho_{\mathrm{o}}}\right) \\
H_{\mathrm{o}} & =b M_{\mathrm{o}}^{\mathrm{n}} T_{\mathrm{o}} \\
H_{\mathrm{a}} & =b M_{\mathrm{a}}{ }^{\mathrm{n}} T_{\mathrm{a}}=b\left(M_{\mathrm{o}} \frac{\rho_{\mathrm{a}}}{\rho_{\mathrm{o}}}\right)^{\mathrm{n}} T_{\mathrm{a}}=H_{\mathrm{o}}\left(\frac{\rho_{\mathrm{a}}}{\rho_{\mathrm{o}}}\right)^{\mathrm{n}}\left(\frac{T_{\mathrm{a}}}{T_{\mathrm{o}}}\right) \\
R_{\mathrm{a}} & =R_{\mathrm{o}}\left(\frac{\rho_{\mathrm{a}}}{\rho_{\mathrm{o}}}\right) \\
P_{\mathrm{a}} & =\left(R_{\mathrm{a}}+\frac{w}{r}\right) \frac{S}{c} \\
F & =\frac{H_{\mathrm{a}}}{P_{\mathrm{a}}}
\end{aligned}
$$

where

$M=$ air flow (mass per unit frontal area per unit time),

$H=$ heat dissipated per unit frontal area per unit time,

$R=$ head resistance per unit frontal area,

$P=$ power absorbed,

$T=$ temperature difference between air and water,

$F=$ figure of merit,

$\rho=$ air density,

$w=$ weight of core and contained water,

$r=$ lift-drift ratio,

$S=$ flying speed,

$c=$ a conversion factor ( 0.00267 for the English units),

$b, n=$ constants of the radiator.

In the equations above it will be noted that the performance of a radiator at any altitude in terms of its performance under standard test conditions at ground level at the same flying speed is computed by using two factors, $\rho_{\mathrm{a}} / \rho_{\mathrm{o}}$, a density factor, and $T_{\mathrm{a}} / T_{\mathrm{o}}$, a temperature factor. These factors, computed for each altitude are shown in Figs. 46 and 47 . Since the atmosphere grows colder as altitude increases, the temperature difference 
between the air and a given water temperature for the radiator becomes greater and the temperature factor increases. The factor does not start with unity at ground level, because average weather conditions impose a temperature difference somewhat greater than the standard test difference of $100^{\circ} \mathrm{F}\left(55.6^{\circ} \mathrm{C}\right)$.

99. EFFects of Altitude Conditions on Properties.-Fig. 47 , showing the ratio of density at altitudes to density at the ground, also represents the variation of both air flow and head

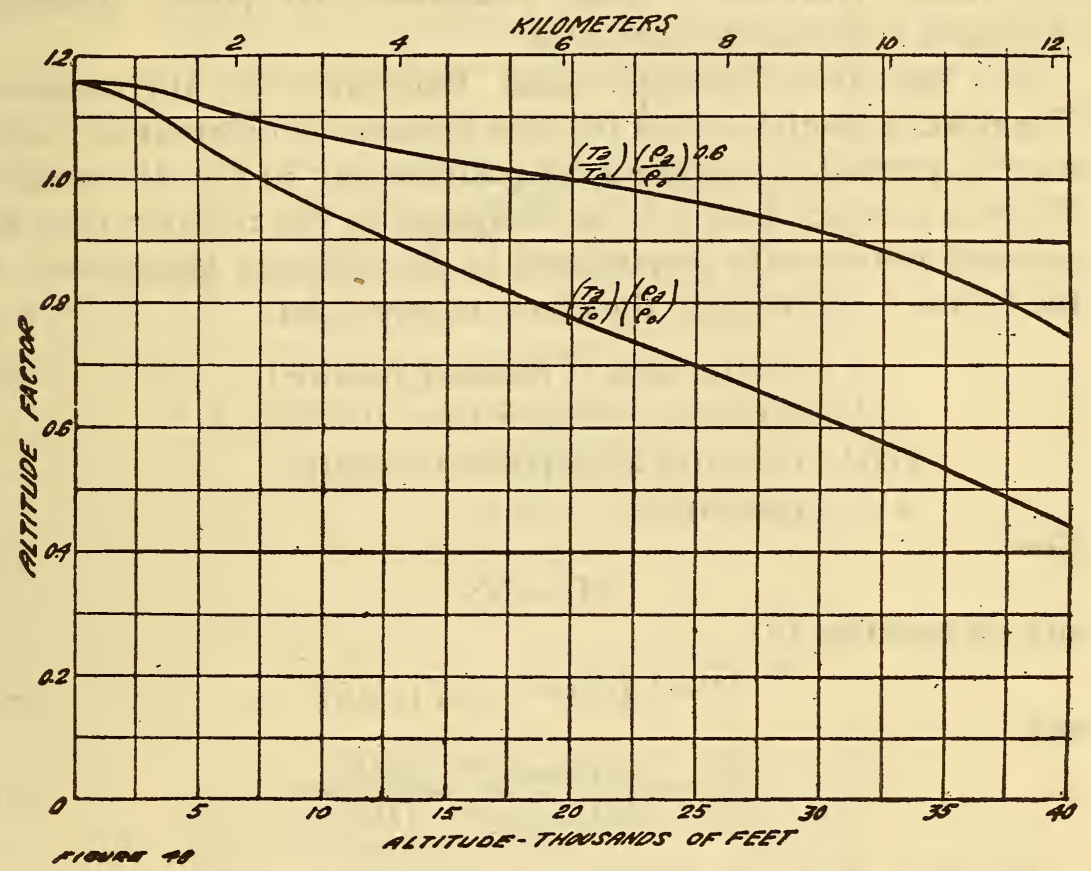

Fig. 48.- Heat dissipation factor for altitude

The heat dissipation of the radiator under conditions prevailing at altitude is computed from the heat dissipation under normal test conditions of air density $0.075 \mathrm{lbs}$. per $\mathrm{ft}^{3}$ and temperature difference $100^{\circ} \mathrm{F}$ between entrance air and average water temperatures, by multiplying by the factor plotted above. Factors are plotted for two very different types of radiator, $n=1 . \infty$ and $\circ 6 \circ$, respectively, in the empirical equation $H=b M^{n} T$

resistance with altitude for a given flying speed. (The curves are nearly identical for summer, winter, and annual means, and only that based on the annual mean is plotted.)

The variation of heat dissipation with altitude at a given flying speed depends by equation (3) both upon the exponent $n$ of the empirical equation applying to the radiator and upon the maximum allowable temperature of the water. The curves of Fig. 48 are computed on the assumption of mean annual conditions of the atmosphere and the requirement that the mean temperature of $75013^{\circ}-22-8$ 
the water in the radiator shall be $30^{\circ} \mathrm{F}$ below the boiling point, ${ }^{27}$ and for such types of core that the exponent $n$ takes the values I.oo and 0.60 .

The effect of altitude on figure of merit at given flying speeds is shown in Fig. 49 for three typical radiators, computed from the above equations and the properties of the radiators at the ground. These values are for unobstructed positions and a lift-drift ratio of 5.4. The above equations apply, however, to obstructed and slip-stream positions if head resistance and power absorbed chargeable to the radiator are used.

ioo. Relative Frontal AREa Reguired at Altitudes.The relative frontal area of radiator required at different altitudes may be estimated as follows for positions not in the slip stream. The rate at which heat is to be dissipated by the radiator may be assumed to be nearly proportional to the indicated horsepower of the engine. ${ }^{28} \quad$ Extending the above notation, let

$A=$ frontal area of radiator required,

$H^{\prime}=$ total heat transfer from the area $A$,

$I H P=$ indicated horsepower of engine, $a^{\prime}, b=$ constants.

Now

and by equation (2)

$$
M=a^{\prime} \rho S
$$

$$
H^{\prime}=b A T M^{\mathrm{n}}=b A T\left(a^{\prime} \rho S\right)^{\mathrm{n}}
$$

and

$$
\frac{H^{\prime}{ }_{\mathrm{a}}}{{H^{\prime}}_{\mathrm{o}}}=\frac{A_{\mathrm{a}} T_{\mathrm{a}}\left(\rho_{\mathrm{a}} S_{\mathrm{a}}\right)^{\mathrm{n}}}{A_{\mathrm{o}} T_{\mathrm{o}}\left(\rho_{\mathrm{o}} S_{\mathrm{o}}\right)^{\mathrm{n}}}=\frac{I H P_{\mathrm{a}}}{I H P_{\mathrm{o}}}
$$

Therefore, the ratio of the area required at an altitude to that required at the ground is given by

$$
\frac{A_{\mathrm{a}}}{A_{\mathrm{o}}}=\frac{I H P_{\mathrm{a}}}{I H P_{\mathrm{o}}}\left(\frac{T_{\mathrm{o}}}{T_{\mathrm{a}}}\right)\left(\frac{\rho_{\mathrm{o}}}{\rho_{\mathrm{a}}}\right)^{\mathrm{n}}\left(\frac{S_{\mathrm{o}}}{S_{\mathrm{a}}}\right)^{\mathrm{n}}
$$

The factors $\frac{T_{\mathrm{a}}}{T_{\mathrm{o}}}$ and $\frac{\rho_{\mathrm{a}}}{\rho_{\mathrm{o}}}$ may be read from Figs. 46 and 47 , while the factors $\frac{S_{\mathrm{o}}}{S_{\mathrm{a}}}$ and $\frac{I H P_{\mathrm{a}}}{I H P_{\mathrm{o}}}$ are obtained from the performance of the engine and plane.

\footnotetext{
${ }^{27}$ This assumption is entirely arbitrary. It may be noted, also, that it determines the starting point of the temperature factor curve of Fig. 46 , giving $182^{\circ} \mathrm{F}$ as the water temperature; mean weather temperature is $+67^{\circ} \mathrm{F}$, a difference of $I 15^{\circ} \mathrm{F}$ as against $100^{\circ} \mathrm{F}$ standard test temperature difference.

${ }^{23}$ Unless affected by such abnormal conditions as heat leak from the turbine of a supercharger or of the exhaust manifold to the cylinder jackets.
} 

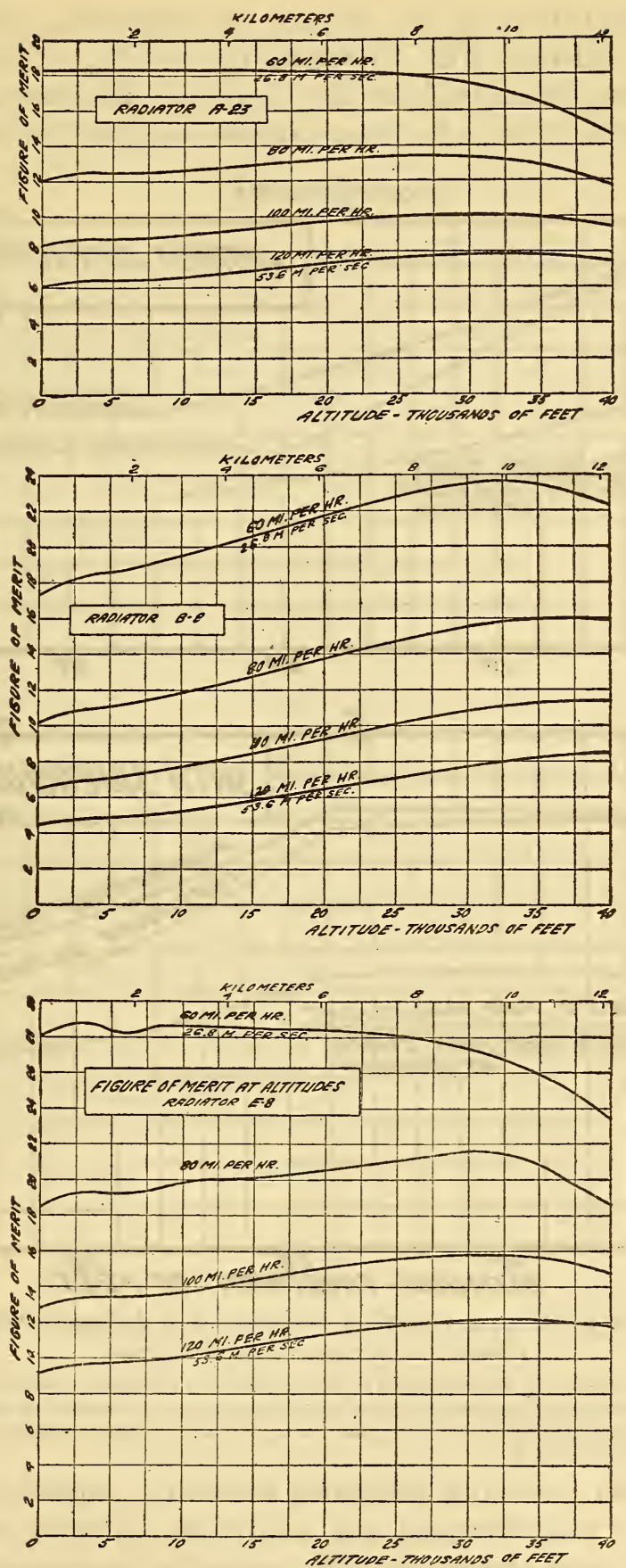

FIG. 49. - Figures of merit for typical radiators at altitude, computed from mean annual weather data for the United States and from test data under ground conditions

The curves correspond to the arbitrary specification that at each altitude the radiator size is so chosen as to maintain the mean temperature of the water in the radiator at $30^{\circ} \mathrm{F}$ below the boiling point 
As an illustration of the method of computing radiator area required at altitude, Fig. 50 shows the results of computations based on data submitted to the National Advisory Committee for Aeronautics ${ }^{29}$ for a Le Pere biplane equipped with variable-

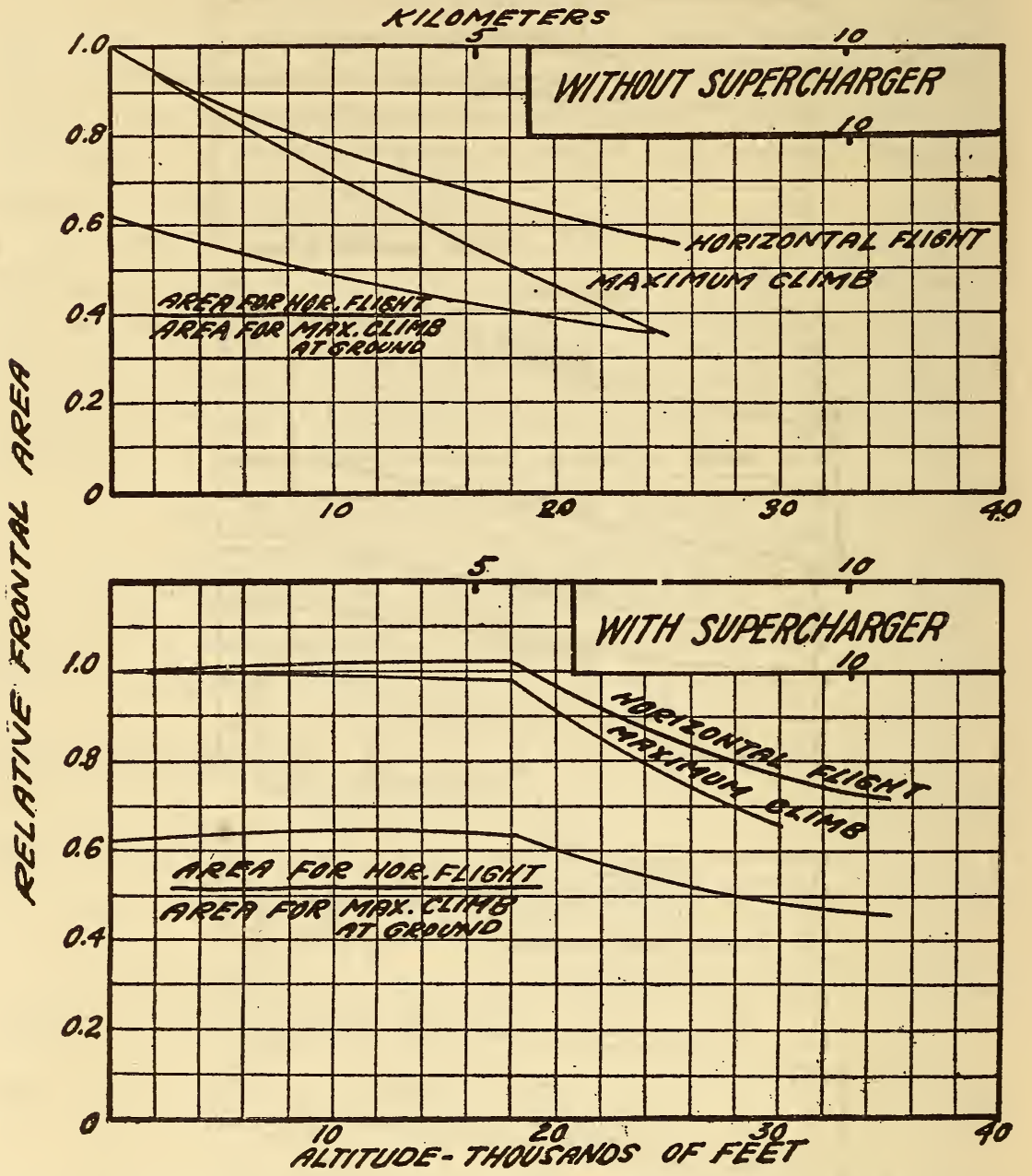

FIG. 50.-Relative frontal area of radiator core required at different altitudes, for unobstructed position not in the slip stream

Computations are based on the data shown in Figs 46 and 47 for atmospheric conditions at altitude. The airplane characteristics assumed include a lift drift ratio of 5.4, a variable pitch propeller controlled so as to hold engine speed always at $1800 \mathrm{r}$. p. m., and a supercharger designed for critical altitude of 5.5 kilometers ( $18000 \mathrm{ft}$.)

pitch propeller and both with and without a supercharger. The supercharger was designed for a critical altitude of 5500 in (I 8 ooo feet), and the pitch of the propeller was assumed so controlled that the speed of the engine was maintained constant at I 800 r. p. m. 


\section{METHODS OF SHUTTERING}

ior. Detachable Sections.-Change of radiator capacity by the use of detachable sections is good for seasonal corrections such as summer and winter conditions, and for change from one latitude to another.

IO2. RETRACTABLE TYPES.-Retractable types will allow withdrawing the radiator or a part of it into the fuselage when its full cooling capacity is not needed. This is one of the best methods from the standpoint of heat dissipation and head resistance, for only the part of the radiator really required for use need be outside of the fuselage where it will affect the resistance of the plane. Difficulties may be encountered, however, in designing the fuselage for such an installation.

IO3. SidE RADIATORS.-Two radiators may be used, one on each side of the fuselage, fitted with shutters that may be two plates for each radiator, hinged at the sides of the fuselage and swinging out to mask the front and rear faces to any desired extent. This method gives approximately the advantages of an unobstructed position, except for the slip stream, and avoids some of the difficulties in design encountered with the retractable type.

IO4. WINDOW-BLIND ShUTTERS.-Shutters of the window-blind type are objectionable because they add greatly to the resistance of the radiator when closed, and their effect in increasing head resistance and decreasing air flow is by no means negligible when open..$^{30}$

I05. Pivoted TyPes. - Some attempts have been made to use a radiator pivoted about a vertical axis, so that it can be swung into or out of the air stream. This method is unsatisfactory, because when the radiator is turned about a vertical axis or yawed the heat dissipation may even be increased rather than diminished, while the head resistance will in general be increased. The method not only fails to give satisfactory control of cooling capacity, but adds considerably to the absorption of power.

io6. Regulation of WATER Flow.-Control by regulation of the water flow has been suggested, but no satisfactory method has been proposed. If the water is cut off from a part of the radiator, that part is likely to freeze at great altitudes or in cold weather, while a change in the rate of flow is not practicable, because with the rates commonly used in practice the heat dissipated is almost independent of the rate of flow.

\footnotetext{
${ }^{30}$ Since for a radiator in the nose of the fuselage, a decrease in air flow is accompanied by a decrease in head resistance chargeable to the radiator; shuttering a nose radiator decreases the resistance of the entire plane.
} 
I07. Stream-Line Casing.- If a stream-line casing for the radiator should be found useful, it would seem to be a simple problem to stream line the core in one dimension and the headers in the other, to arrange the stream line sides to open or close, and to put the mechanism for controlling them inside of the streamline casing of the headers.

\section{GENERAL TABLES AND PLOTS}

i08. Gejometrical Characteristics of Radiator.-In the following tables and plots the geometrical characteristics and physical properties of the radiator cores measured are collected for reference. There are a number of specimens on which for various reasons complete measurements were not made, and these have been separated from the others in the tables.

Table 20 shows geometrical characteristics and Table 2 I shows physical properties, and a series of graphs show the performance of the different types of radiators. All performance figures as given apply to unobstructed positions, but the relation between heat dissipation and air flow is independent of the position.

\section{CLASSIFICATION OF CORES}

The laboratory classification of the cores was accidental rather than logical, but the general underlying subdivision is as follows, a good many exceptions occurring which can only be identified by the headings of Table 20 or by Figs $5^{\mathrm{I}-\mathrm{I} 2 \mathrm{I}}$ :

$$
\begin{array}{ll}
\mathrm{A}=\text { Square cell } & \mathrm{E}=\text { flat plate } \\
\mathrm{B}=\text { hexagonal } & \mathrm{F}=\text { fin and tube } \\
\mathrm{C}=\text { circular } & \mathrm{G}=\text { miscellaneous. }
\end{array}
$$




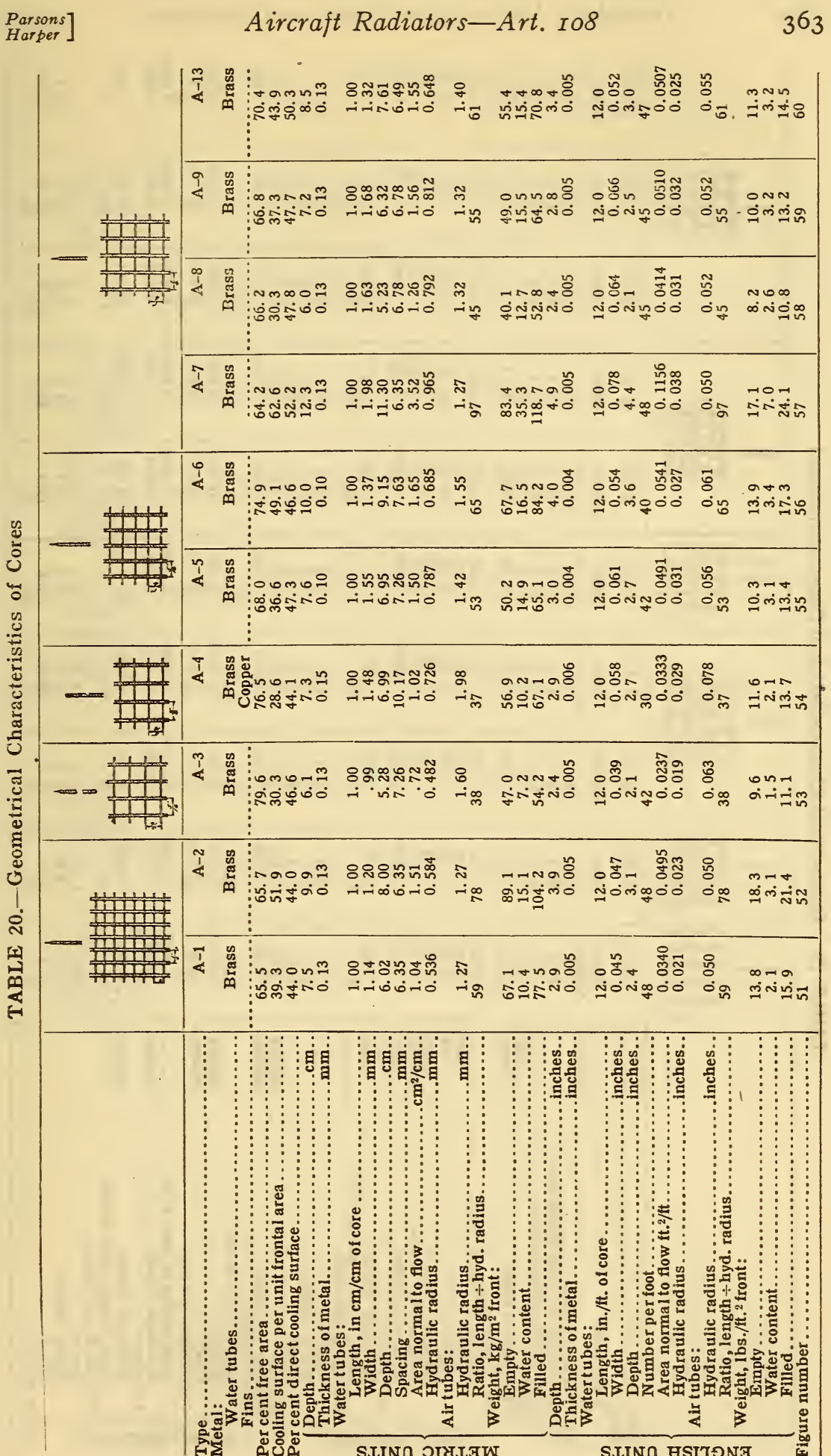




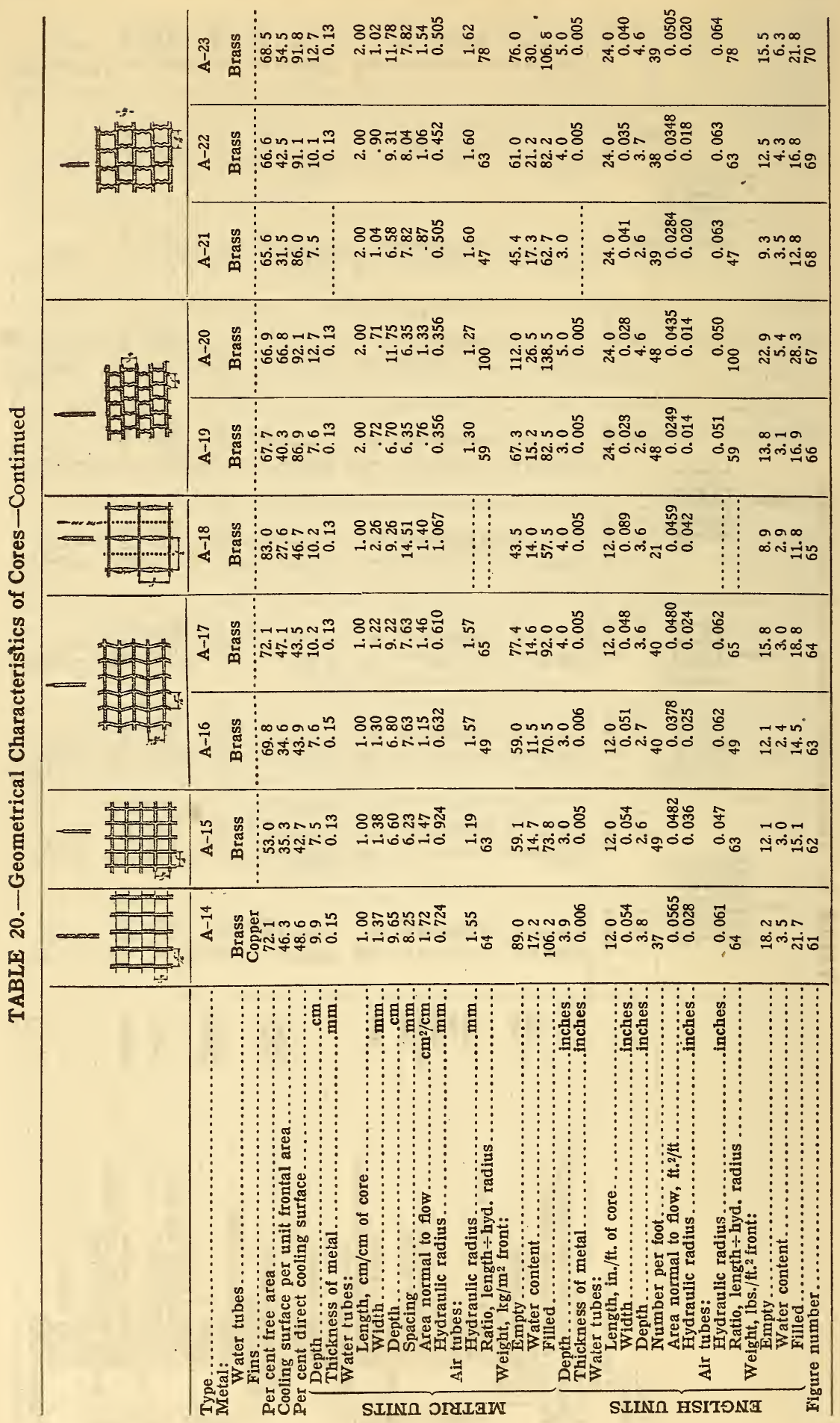




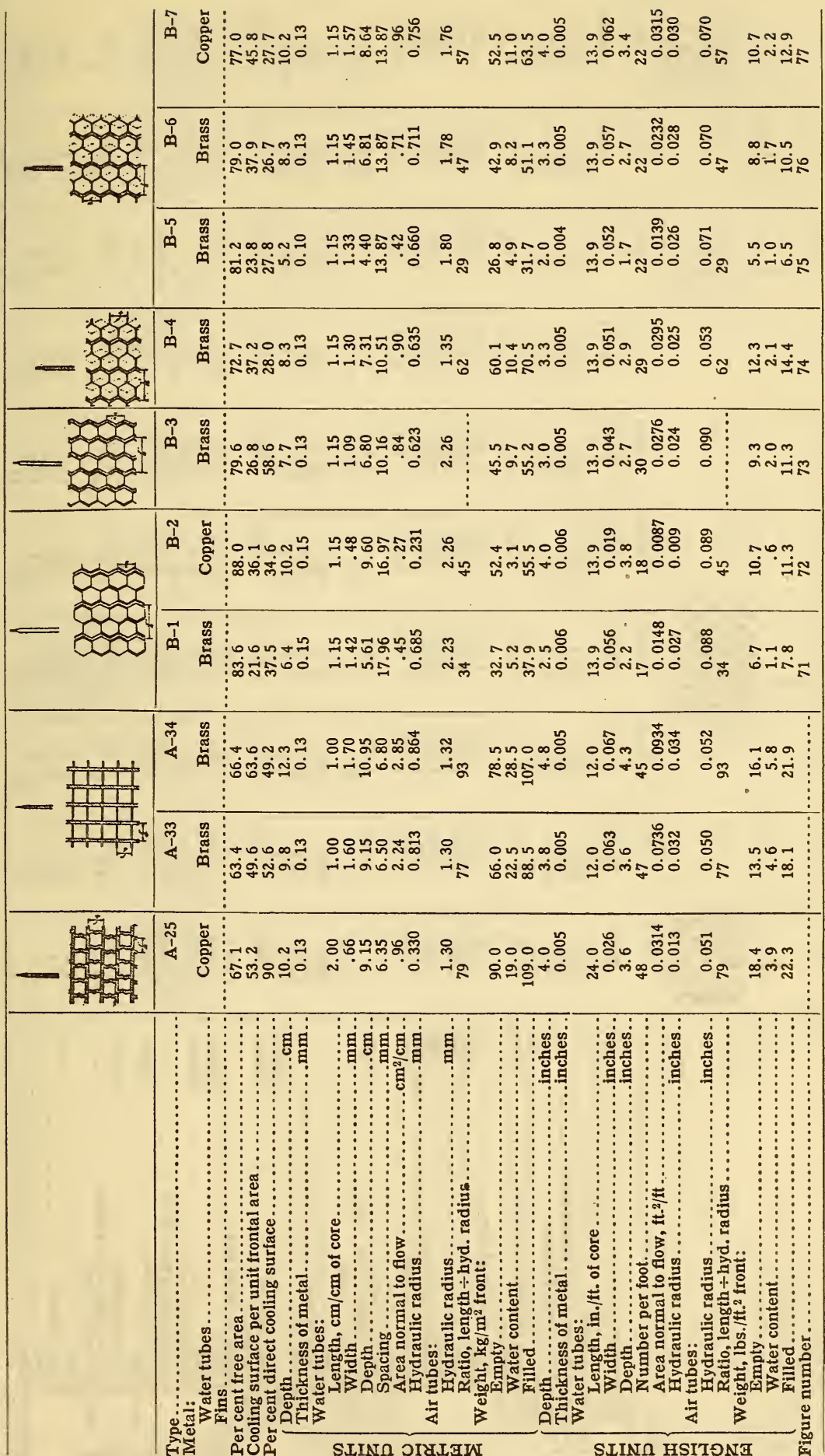




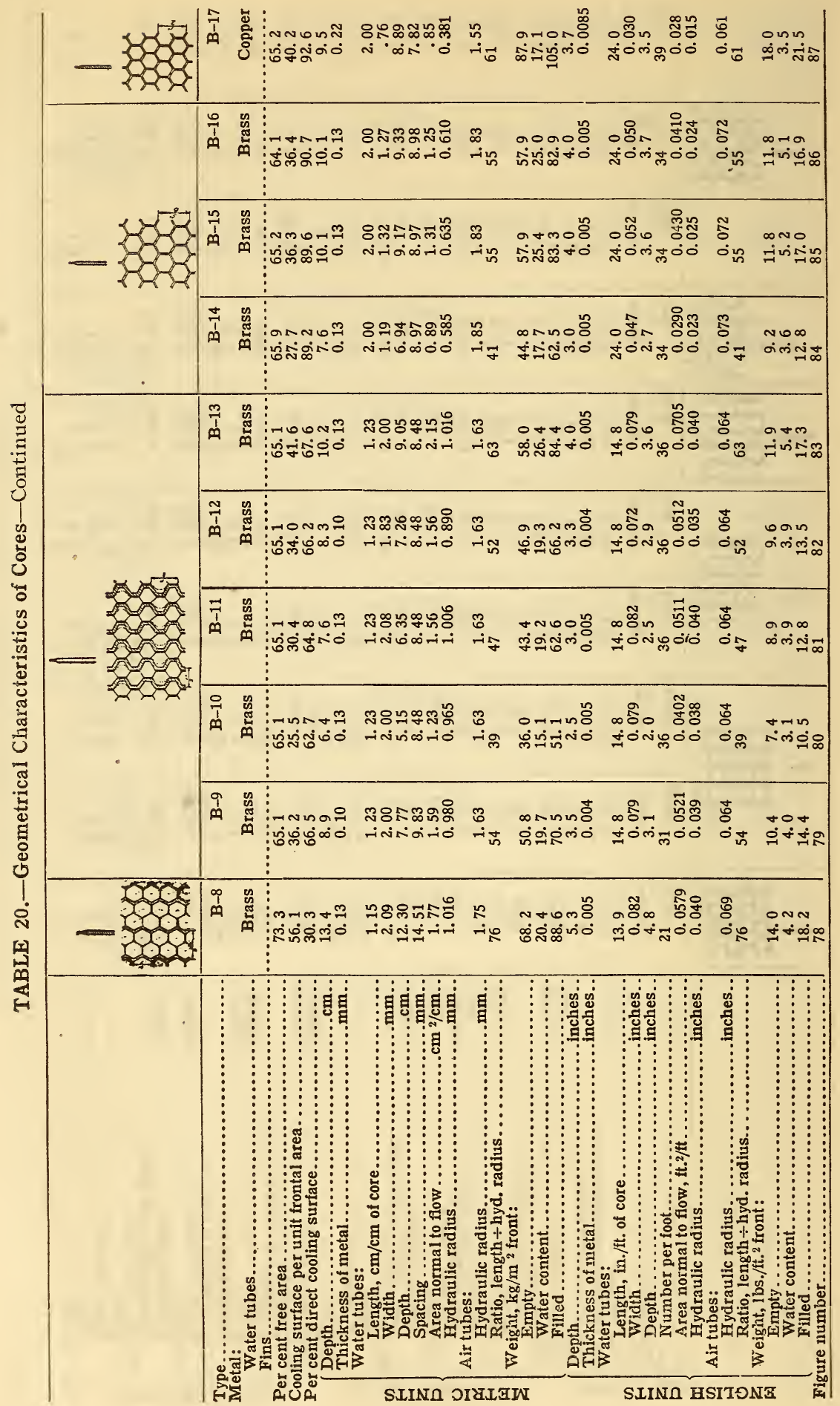




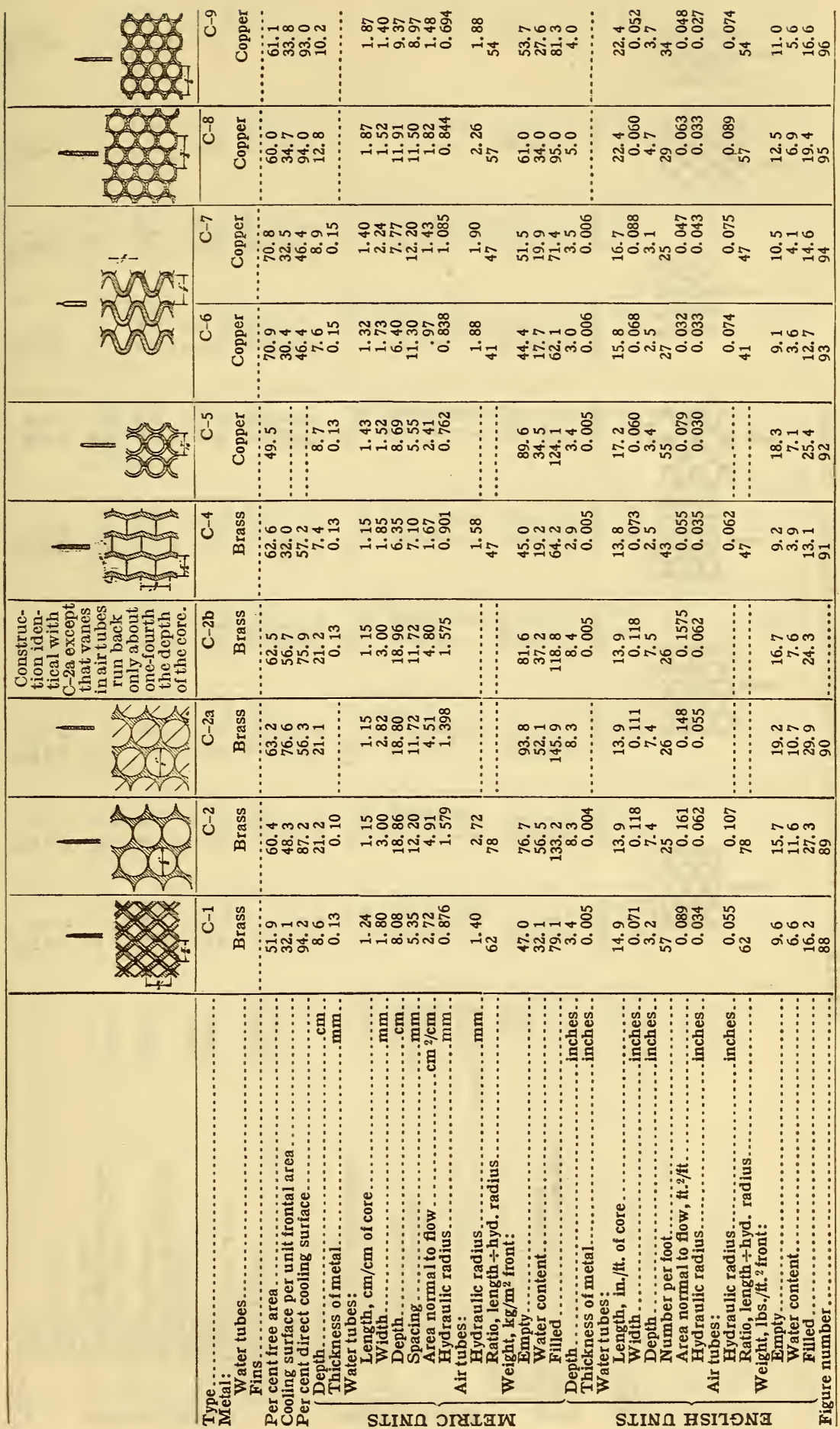




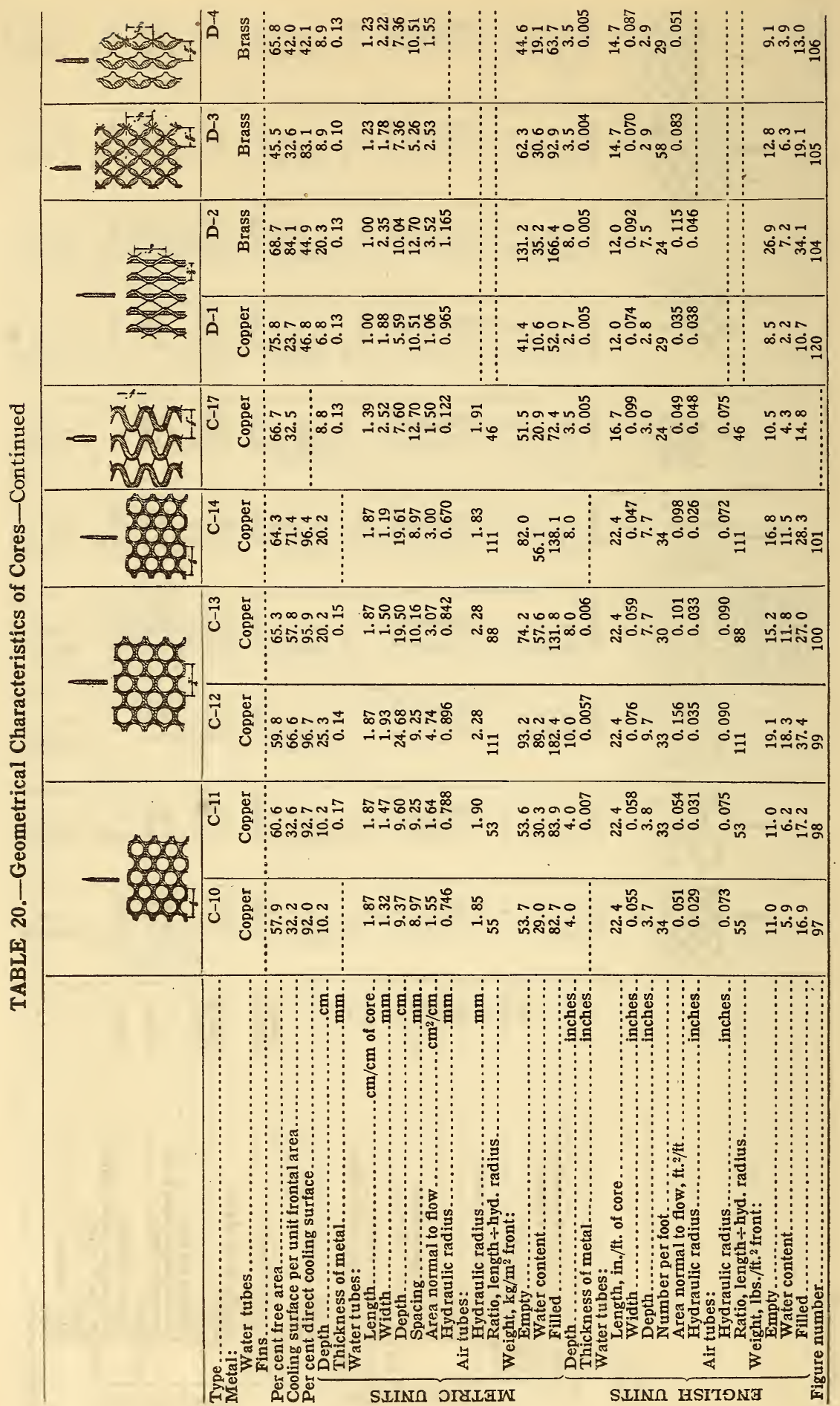




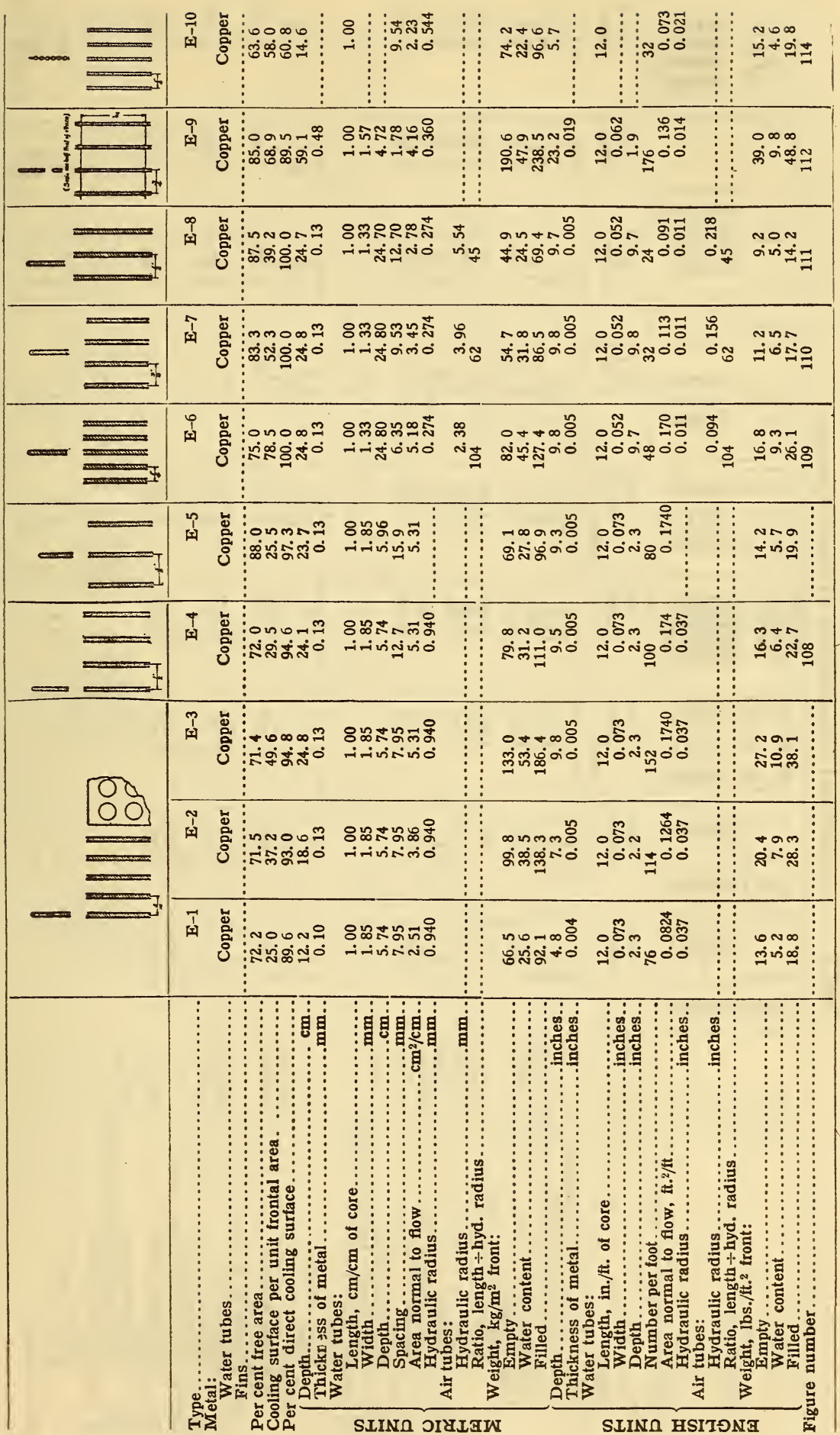




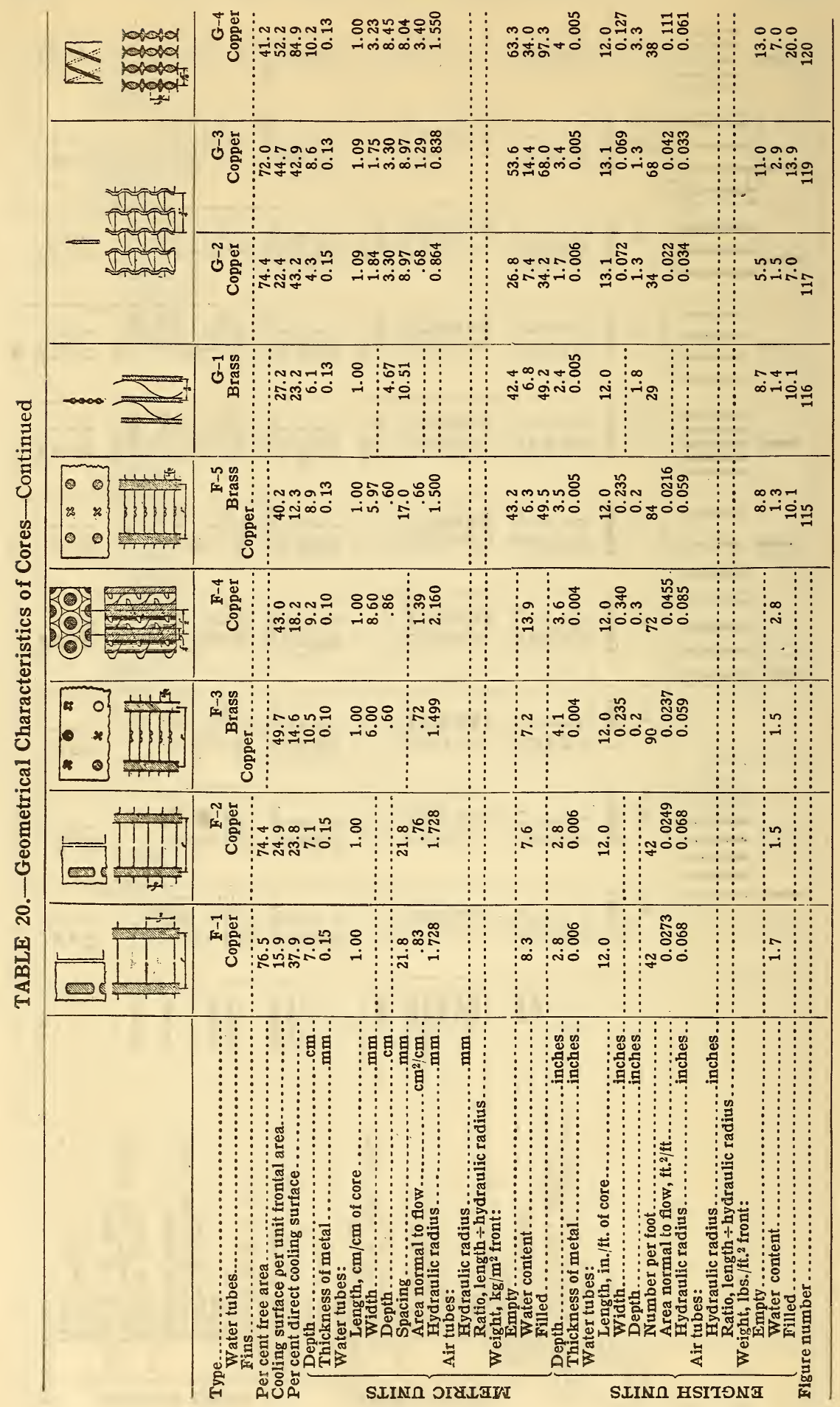




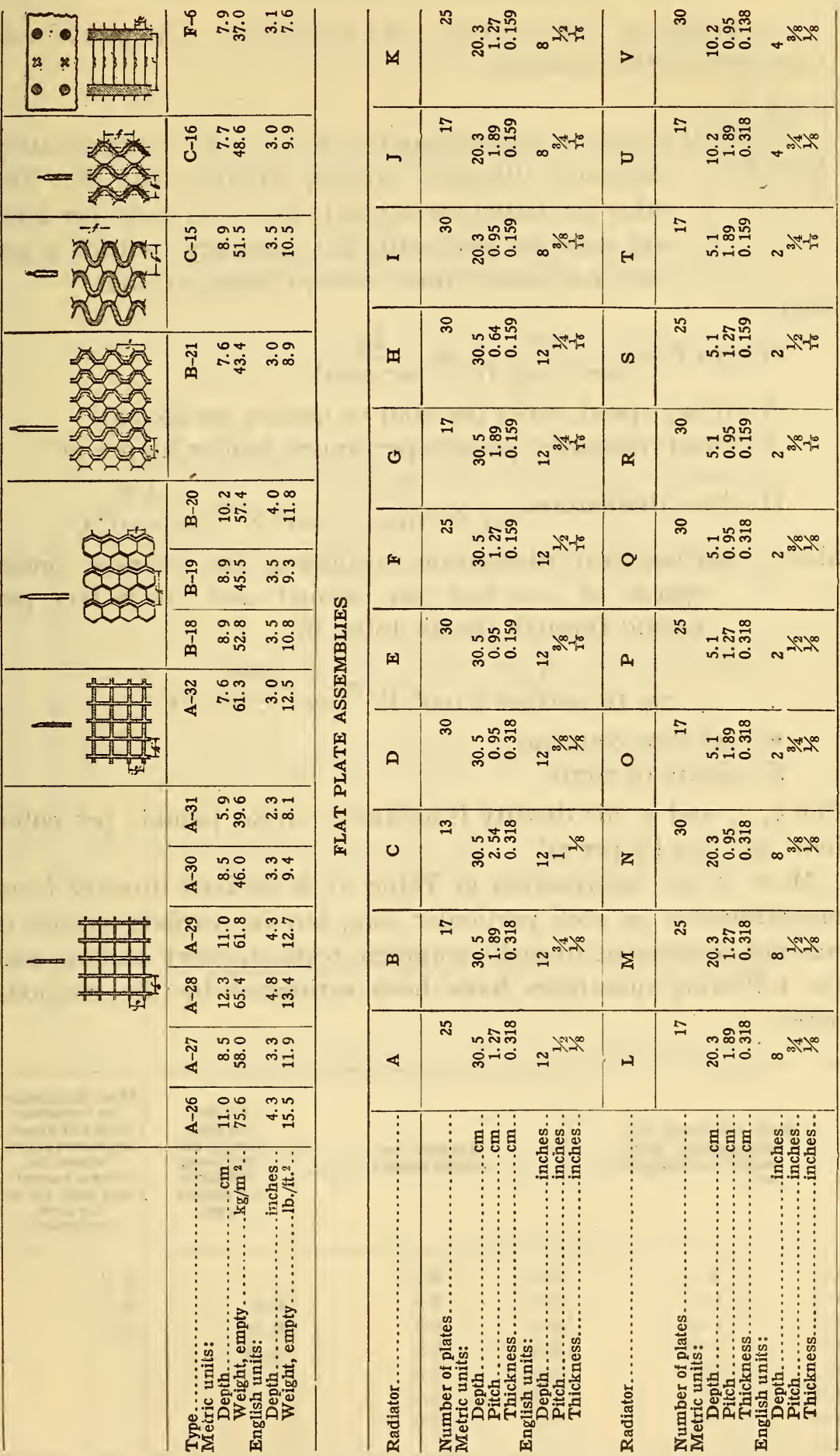


io9. Physical Properties of Radiators.-Empirical constants are for the equations

$M=k S$

$H=b M^{\mathrm{n}}\left(R\right.$ is nearly proportional to $S^{2}$ or $c$ is approximately $R=c S^{2} \quad$ constant, although varying slightly with $S$. The value tabulated corresponds to $S=60$ miles per hour and may be used with fair accuracy (within 5 per

where cent) for speeds from 40 to 90 miles per hour,

$M=$ air flow, $\frac{\mathrm{lb} \text {. }}{\text { sec. } \times \text { sq. } \mathrm{ft} \text {. }}$ or $\frac{\mathrm{kg}}{\mathrm{sec} . \times \mathrm{m}^{2}}$

$S=$ flying speed, miles per hour or meters per second,

$R=$ head resistance, pounds per square foot or $\mathrm{kg}$ per $\mathrm{m}^{2}$

$H=$ heat dissipation, $\frac{\mathrm{h} . \mathrm{p} .}{\mathrm{sq} \text {. ft. front } \times 100^{\circ} \mathrm{F}}$ or $\frac{\mathrm{kw}}{\mathrm{m}^{2} \times 50^{\circ} \mathrm{C}}$

also $q=$ surface heat dissipation coefficient for average linear speeds of 100 feet per second and 30 meters per second through the air tubes in

$$
\frac{\text { h. p. }}{\text { sq. ft. surface } \times 100^{\circ} \mathrm{F}} \text { or } \frac{\mathrm{cal}}{\sec \times \mathrm{cm}^{2} \times{ }^{\circ} \bar{C}}
$$

$m=$ air flow constant,

$F=$ figure of merit.

For $k, c$, and $q$, air density is assumed 0.0750 pounds per cubic foot, or $1.204 \mathrm{~kg}$ per $\mathrm{m}^{3}$.

Most of the information in Table $2 \mathrm{I}$ is derived directly from measurements on each particular core, but for various reasons it was not convenient to make complete tests on every section, and the following quantities have been estimated by the methods noted:

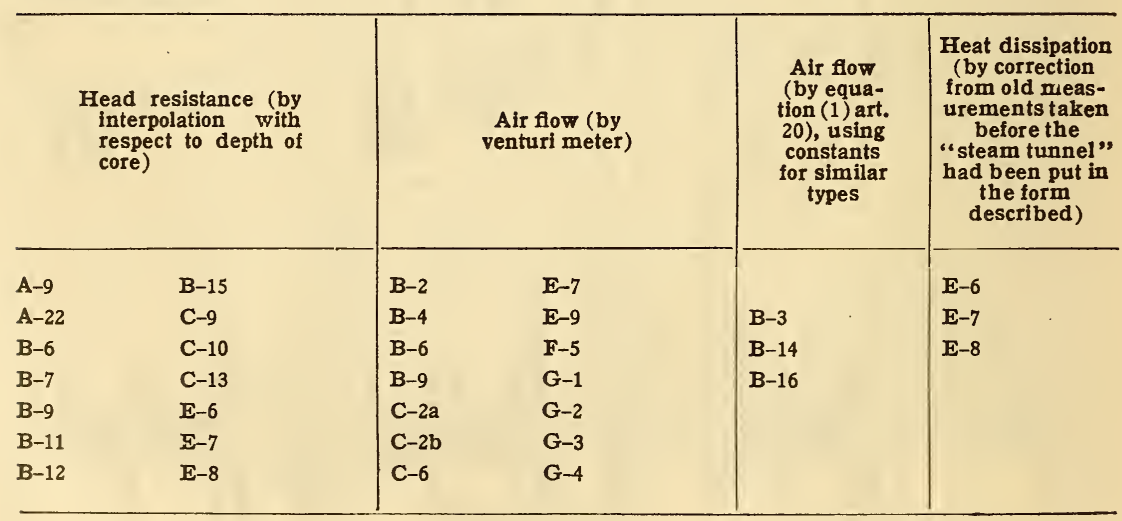




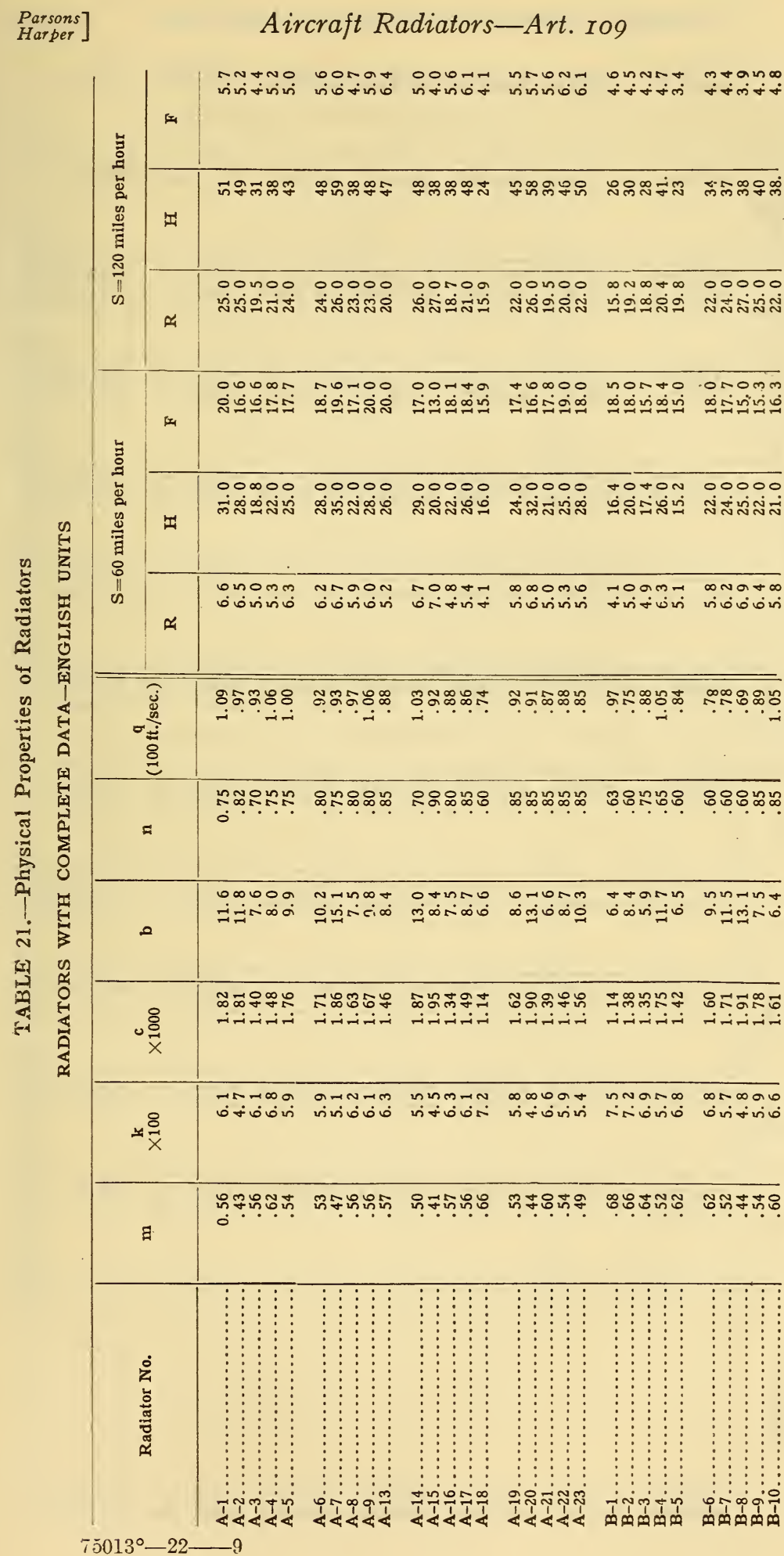




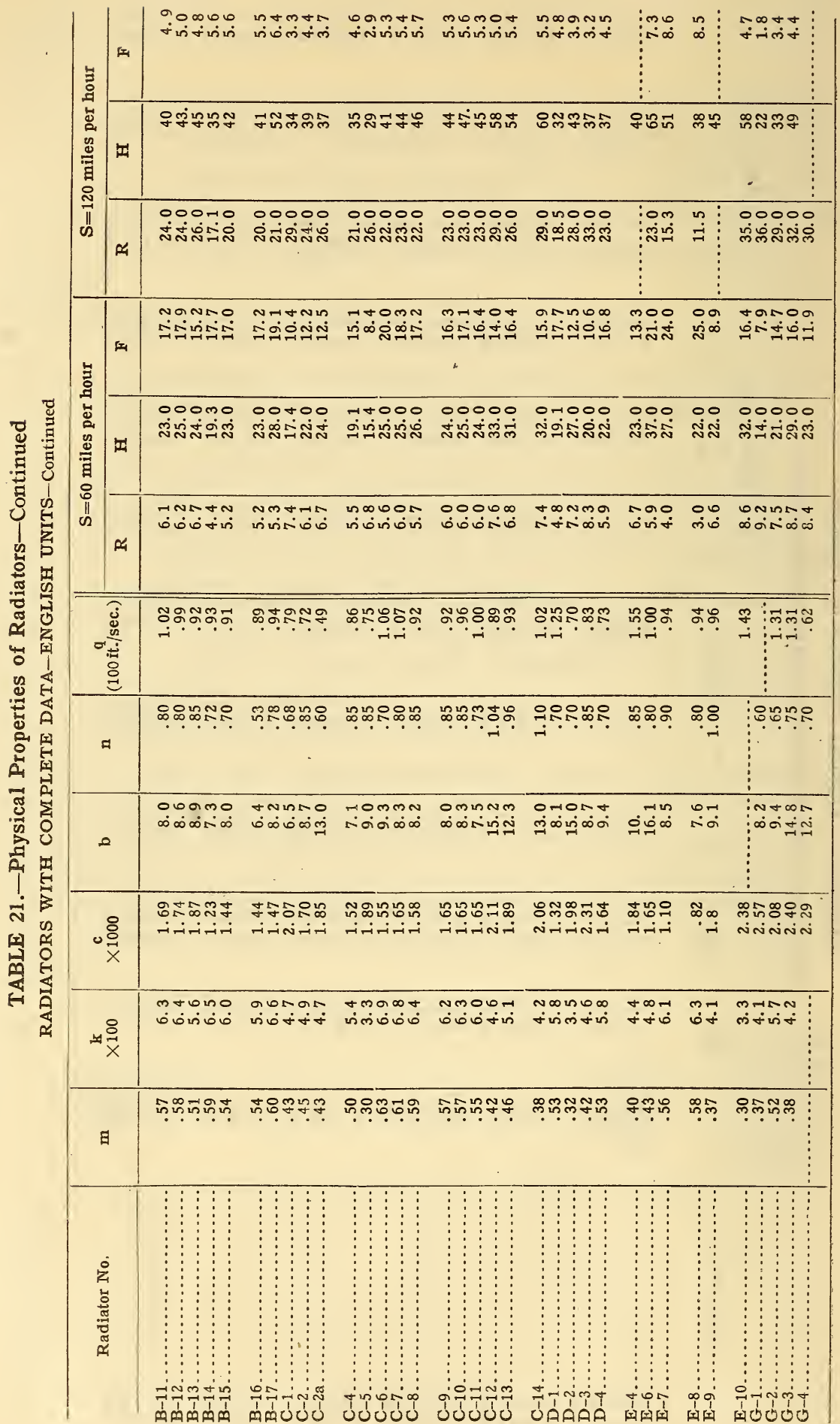


TABLE 21.-Physical Properties of Radiators-Continued RADIATORS WITH INCOMPLETE DATA-ENGLISH UNITS

\begin{tabular}{|c|c|c|c|c|c|c|}
\hline Radiator No. & $\mathbf{m}$ & $\times \frac{\mathbf{k}}{100}$ & $\begin{array}{l}\text { c } \\
\times 1000\end{array}$ & b & $\mathbf{n}$ & $(100 \mathrm{ft} . / \mathrm{sec})$. \\
\hline $\begin{array}{l}A-25 \\
A-26 \\
A-27 \\
A-28 \\
A-29\end{array}$ & $\begin{array}{l}0.50 \\
.55 \\
.56 \\
.59 \\
.55\end{array}$ & $\begin{array}{l}5.5 \\
6.0 \\
6.2 \\
6.5 \\
6.1\end{array}$ & $\begin{array}{l}1.70 \\
1.80 \\
1.85 \\
1.74 \\
1.54\end{array}$ & 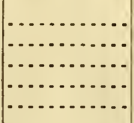 & 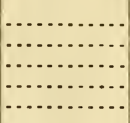 & (2. \\
\hline $\begin{array}{l}A-30 \\
A-51 \\
A-32 \\
A-33 \\
B-18\end{array}$ & $\begin{array}{l}.59 \\
.59 \\
.54 \\
.5 \\
. .\end{array}$ & \begin{tabular}{l}
6.4 \\
6.5 \\
\hdashline 6.0 \\
$\cdots \cdots$
\end{tabular} & $\begin{array}{r}1.52 \\
1.35 \\
1.45 \\
1.24\end{array}$ & 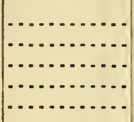 & $\begin{array}{ll} \\
\cdots \cdots \\
\cdots \cdots\end{array}$ & $\begin{array}{ll} \\
\cdots\end{array}$ \\
\hline $\begin{array}{l}\text { B-19. } \\
\text { B-20 } \\
\text { B-21 } \\
\text { C-2b. } \\
\text { C- } 15 . \ldots\end{array}$ & (45 & $\begin{array}{l}\cdots \\
\cdots \\
5.0 \\
\cdots\end{array}$ & $\begin{array}{r}1.13 \\
1.00 \\
1.86 \\
1.46\end{array}$ & 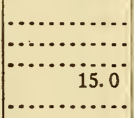 & $\begin{array}{c}\ldots \ldots \ldots \\
\cdots \ldots \ldots \\
\cdots \\
\cdots\end{array}$ & 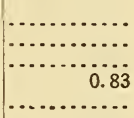 \\
\hline 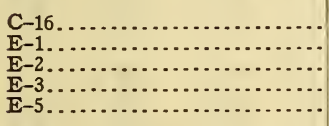 & .36 & $\begin{array}{l}3.9 \\
7.0\end{array}$ & 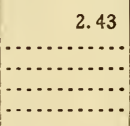 & 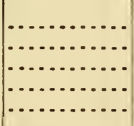 & $\begin{array}{ll} \\
\cdots\end{array}$ & $\begin{array}{r}2.25 \\
1.85 \\
1.80 \\
\ldots . . .\end{array}$ \\
\hline $\begin{array}{l}\mathrm{F}-1 \\
\mathrm{~F}-2 \\
\mathrm{~F}-5 \\
\mathrm{~F}-6.2\end{array}$ & $\begin{array}{l}.65 \\
.36 \\
.34 \\
.\end{array}$ & $\begin{array}{l}7.1 \\
3.9 \\
3.7 \\
-3\end{array}$ & 2.68 & $\begin{array}{l} \\
\cdots \\
\cdots\end{array}$ & (2) & $\begin{array}{l}\cdots \\
\cdots \\
\cdots \\
\cdots\end{array}$ \\
\hline
\end{tabular}

RADIATORS WITH INCOMPLETE DATA-METRIC UNITS

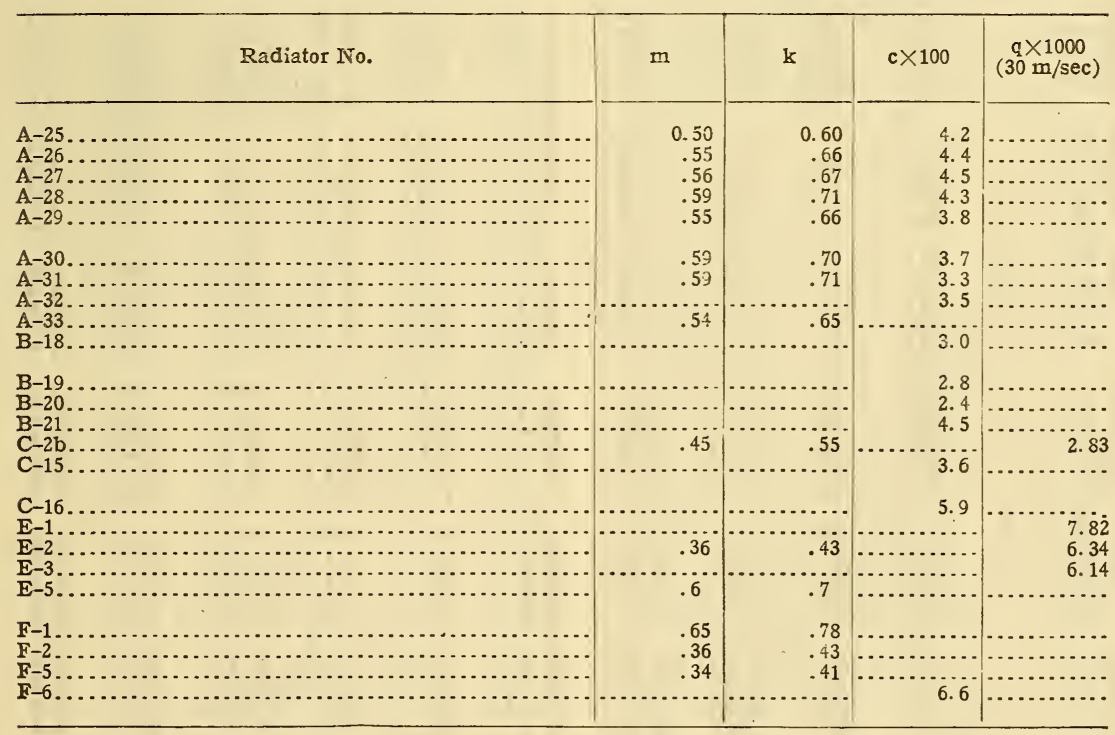


TABLE 21.-Physical Properties of Radiators-Continued

RADIATORS WITH COMPLETE DATA-METRIC UNITS

\begin{tabular}{|c|c|c|c|c|c|c|c|c|c|c|}
\hline \multirow{2}{*}{$\begin{array}{l}\text { Radiator } \\
\text { No. }\end{array}$} & \multirow{2}{*}{$\mathrm{m}$} & \multirow{2}{*}{$\mathbf{k}$} & \multirow{2}{*}{$\mathbf{c} \times 100$} & \multirow{2}{*}{$\begin{array}{c}q \times 1000 \\
(30 \mathrm{~m} / \mathrm{sec})\end{array}$} & \multicolumn{3}{|c|}{$\mathrm{S}=25$ meters per sec } & \multicolumn{3}{|c|}{$\mathrm{S}=50$ meters per sec } \\
\hline & & & & & $\mathbf{R}$ & $\mathbf{H}$ & F & $\mathbf{R}$ & $\mathbf{H}$ & $F$ \\
\hline $\begin{array}{l}A-1 \ldots \ldots . \\
A-2 \ldots \ldots . \\
A-3 \ldots \ldots . \\
A-4 \ldots \ldots \\
A-5 \ldots \ldots\end{array}$ & $\begin{array}{l}0.56 \\
.43 \\
.56 \\
.62 \\
.54\end{array}$ & $\begin{array}{l}0.67 \\
.52 \\
.67 \\
.74 \\
.64\end{array}$ & $\begin{array}{l}4.5 \\
4.4 \\
3.4 \\
3.6 \\
4.3\end{array}$ & $\begin{array}{l}3.73 \\
3.29 \\
3.15 \\
3.62 \\
3.40\end{array}$ & $\begin{array}{l}28.4 \\
29.3 \\
21.5 \\
23.5 \\
27.4\end{array}$ & $\begin{array}{l}210 \\
188 \\
132 \\
153 \\
168\end{array}$ & $\begin{array}{l}23.0 \\
18.5 \\
18.7 \\
20.0 \\
20.1\end{array}$ & $\begin{array}{r}108.0 \\
108.0 \\
83.5 \\
87.5 \\
104.0\end{array}$ & $\begin{array}{l}352 \\
334 \\
210 \\
263 \\
294\end{array}$ & $\begin{array}{l}6.4 \\
6.3 \\
5.0 \\
5.8 \\
5.7\end{array}$ \\
\hline $\begin{array}{l}A-6 \ldots \ldots . \\
A-7 \ldots \ldots . \\
A-8 \ldots \ldots . \\
A-9 \ldots \ldots . \\
A-13 \ldots \ldots .\end{array}$ & $\begin{array}{l}.53 \\
.47 \\
.56 \\
.56 \\
.57\end{array}$ & $\begin{array}{l}.64 \\
.56 \\
.68 \\
.67 \\
.69\end{array}$ & $\begin{array}{l}4.2 \\
4.5 \\
4.0 \\
4.1 \\
3.6\end{array}$ & $\begin{array}{l}3.13 \\
3.16 \\
3.29 \\
3.62 \\
2.99\end{array}$ & $\begin{array}{l}26.4 \\
28.4 \\
24.4 \\
26.4 \\
21.5\end{array}$ & $\begin{array}{l}191 \\
240 \\
148 \\
188 \\
180\end{array}$ & $\begin{array}{l}21.0 \\
22.0 \\
19.2 \\
23.3 \\
23.0\end{array}$ & $\begin{array}{r}102.0 \\
110.0 \\
96.0 \\
99.0 \\
87.5\end{array}$ & $\begin{array}{l}330 \\
405 \\
257 \\
330 \\
320\end{array}$ & $\begin{array}{l}6.4 \\
7.0 \\
5.4 \\
6.8 \\
7.2\end{array}$ \\
\hline $\begin{array}{l}A-14 \ldots . . . \\
A-15 \ldots . . \\
A-16 \ldots . . . \\
A-17 \ldots . . \\
A-18 \ldots . . .\end{array}$ & $\begin{array}{l}.50 \\
.41 \\
.57 \\
.56 \\
.66\end{array}$ & $\begin{array}{l}.60 \\
.49 \\
.69 \\
.67 \\
.79\end{array}$ & \begin{tabular}{l|}
4.6 \\
4.8 \\
3.3 \\
3.6 \\
2.8
\end{tabular} & $\begin{array}{l}3.52 \\
3.14 \\
3.00 \\
2.92 \\
2.51\end{array}$ & $\begin{array}{l}29.3 \\
30.5 \\
20.6 \\
22.5 \\
17.6\end{array}$ & $\begin{array}{l}202 \\
140 \\
149 \\
177 \\
108\end{array}$ & $\begin{array}{l}19.0 \\
14.3 \\
20.3 \\
20.2 \\
18.0\end{array}$ & $\begin{array}{r}94.0 \\
116.0 \\
80.0 \\
86.5 \\
68.0\end{array}$ & $\begin{array}{l}316 \\
262 \\
258 \\
322 \\
161\end{array}$ & $\begin{array}{l}6.6 \\
4.5 \\
6.3 \\
6.9 \\
4.7\end{array}$ \\
\hline $\begin{array}{l}A-19 \ldots . . . \\
A-20 \ldots . . \\
A-21 \ldots . . \\
A-22 \ldots . . \\
A-23 \ldots . .\end{array}$ & $\begin{array}{l}.53 \\
.44 \\
.60 \\
.54 \\
.49\end{array}$ & $\begin{array}{l}.64 \\
.52 \\
.72 \\
.65 \\
.60\end{array}$ & $\begin{array}{l}4.0 \\
4.6 \\
3.4 \\
3.6 \\
3.8\end{array}$ & $\begin{array}{l}3.14 \\
3.11 \\
2.96 \\
3.01 \\
2.91\end{array}$ & $\begin{array}{l}25.0 \\
29.9 \\
21.5 \\
22.5 \\
23.5\end{array}$ & $\begin{array}{l}165 \\
217 \\
144 \\
174 \\
190\end{array}$ & $\begin{array}{l}17.3 \\
16.0 \\
17.6 \\
18.7 \\
18.0\end{array}$ & $\begin{array}{r}96.0 \\
112.0 \\
83.0 \\
86.0 \\
93.0\end{array}$ & $\begin{array}{l}305 \\
394 \\
264 \\
312 \\
344\end{array}$ & $\begin{array}{l}5.6 \\
5.9 \\
5.6 \\
6.3 \\
6.1\end{array}$ \\
\hline $\begin{array}{l}\text { B-1 } \ldots \ldots \\
\text { B-2 } \ldots \ldots \\
\text { B-3 } \ldots \ldots \\
\text { B-4 } \ldots \ldots \\
\text { B-5 } \ldots \ldots\end{array}$ & $\begin{array}{l}.68 \\
.66 \\
.64 \\
.52 \\
.62\end{array}$ & $\begin{array}{l}.82 \\
.79 \\
.75 \\
.62 \\
.75\end{array}$ & $\begin{array}{l}2.8 \\
3.4 \\
3.3 \\
4.3 \\
3.5\end{array}$ & $\begin{array}{l}3.29 \\
2.57 \\
3.00 \\
3.59 \\
2.86\end{array}$ & $\begin{array}{l}17.1 \\
21.0 \\
22.0 \\
28.4 \\
22.0\end{array}$ & $\begin{array}{l}110 \\
140 \\
119 \\
183 \\
104\end{array}$ & $\begin{array}{l}21.3 \\
21.0 \\
17.5 \\
20.7 \\
17.0\end{array}$ & $\begin{array}{r}67.0 \\
82.0 \\
82.0 \\
105.0 \\
83.5\end{array}$ & $\begin{array}{l}176 \\
201 \\
196 \\
282 \\
159\end{array}$ & $\begin{array}{l}5.4 \\
5.2 \\
4.6 \\
5.5 \\
3.9\end{array}$ \\
\hline $\begin{array}{l}\text { B-6...... } \\
\text { B-7 ....... } \\
\text { B-8...... } \\
\text { B-9...... } \\
\text { B-10..... }\end{array}$ & $\begin{array}{l}.62 \\
.52 \\
.44 \\
.54 \\
.60\end{array}$ & $\begin{array}{l}.74 \\
.63 \\
.53 \\
.65 \\
.73\end{array}$ & $\begin{array}{l}3.9 \\
4.2 \\
4.7 \\
4.4 \\
3.9\end{array}$ & $\begin{array}{l}2.64 \\
2.67 \\
2.34 \\
3.04 \\
3.57\end{array}$ & $\begin{array}{l}24.0 \\
25.9 \\
31.8 \\
27.9 \\
25.0\end{array}$ & $\begin{array}{l}152 \\
166 \\
171 \\
152 \\
141\end{array}$ & $\begin{array}{l}21.2 \\
20.0 \\
17.8 \\
17.4 \\
18.2\end{array}$ & $\begin{array}{r}94.0 \\
102.0 \\
112.0 \\
106.0 \\
96.0\end{array}$ & $\begin{array}{l}231 \\
253 \\
260 \\
273 \\
255\end{array}$ & $\begin{array}{l}5.2 \\
5.1 \\
4.6 \\
5.4 \\
5.5\end{array}$ \\
\hline $\begin{array}{l}\text { B-11 . . . . } \\
\text { B-12 . . . } \\
\text { B-13..... } \\
\text { B-14 . . . } \\
\text { B-15..... }\end{array}$ & $\begin{array}{l}.57 \\
.58 \\
.51 \\
.59 \\
.54\end{array}$ & $\begin{array}{l}.69 \\
.70 \\
.61 \\
.71 \\
.65\end{array}$ & $\begin{array}{l}4.1 \\
4.3 \\
4.6 \\
3.0 \\
3.5\end{array}$ & $\begin{array}{l}3.47 \\
3.38 \\
3.12 \\
3.18 \\
3.11\end{array}$ & $\begin{array}{l}26.4 \\
26.4 \\
28.4 \\
19.6 \\
23.0\end{array}$ & $\begin{array}{l}158 \\
173 \\
166 \\
132 \\
155\end{array}$ & $\begin{array}{l}19.2 \\
19.9 \\
16.7 \\
20.0 \\
18.6\end{array}$ & $\begin{array}{r}100.0 \\
104.0 \\
111.0 \\
52.0 \\
87.5\end{array}$ & $\begin{array}{l}276 \\
300 \\
306 \\
207 \\
291\end{array}$ & $\begin{array}{l}5.6 \\
5.9 \\
5.4 \\
8.5 \\
6.4\end{array}$ \\
\hline 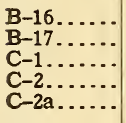 & $\begin{array}{l}.54 \\
.60 \\
.43 \\
.45 \\
.43\end{array}$ & $\begin{array}{l}.65 \\
.72 \\
.52 \\
.54 \\
.51\end{array}$ & $\begin{array}{l}3.5 \\
3.7 \\
5.1 \\
4.2 \\
4.5\end{array}$ & $\begin{array}{l}3.04 \\
3.21 \\
2.69 \\
2.44 \\
1.67\end{array}$ & $\begin{array}{l}22.5 \\
22.3 \\
32.3 \\
26.4 \\
29.8\end{array}$ & $\begin{array}{l}159 \\
192 \\
116 \\
148 \\
169\end{array}$ & $\begin{array}{l}18.8 \\
18.7 \\
11.5 \\
13.4 \\
13.7\end{array}$ & $\begin{array}{r}86.5 \\
91.0 \\
123.0 \\
100.0 \\
111.0\end{array}$ & $\begin{array}{l}280 \\
349 \\
226 \\
264 \\
257\end{array}$ & $\begin{array}{l}6.3 \\
6.4 \\
3.7 \\
4.7 \\
4.5\end{array}$ \\
\hline 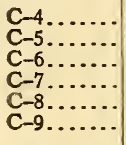 & $\begin{array}{l}.50 \\
.30 \\
.63 \\
.61 \\
.59 \\
.57\end{array}$ & $\begin{array}{l}.60 \\
.36 \\
.76 \\
.74 \\
.71 \\
.68\end{array}$ & $\begin{array}{l}3.7 \\
4.6 \\
3.8 \\
4.0 \\
3.9 \\
4.0\end{array}$ & $\begin{array}{l}2.95 \\
2.56 \\
3.63 \\
3.66 \\
3.14 \\
3.12\end{array}$ & $\begin{array}{l}23.5 \\
30.3 \\
24.4 \\
25.9 \\
23.5 \\
25.4\end{array}$ & $\begin{array}{l}128 \\
110 \\
174 \\
177 \\
170 \\
157\end{array}$ & $\begin{array}{r}16.8 \\
8.7 \\
22.9 \\
20.3 \\
18.8 \\
18.2\end{array}$ & $\begin{array}{r}91.0 \\
121.0 \\
90.0 \\
99.0 \\
93.0 \\
98.0\end{array}$ & $\begin{array}{l}235 \\
199 \\
278 \\
304 \\
315 \\
301\end{array}$ & $\begin{array}{l}5.1 \\
3.2 \\
6.4 \\
5.9 \\
6.4 \\
5.9\end{array}$ \\
\hline $\begin{array}{l}\mathrm{C}-10 \ldots . . \\
\mathrm{C}-11 \ldots . \\
\mathrm{C}-12 \ldots . . \\
\mathrm{C}-13 \ldots . \\
\mathrm{C}-14 \ldots \ldots\end{array}$ & $\begin{array}{l}.57 \\
.55 \\
.42 \\
.46 \\
.38\end{array}$ & $\begin{array}{l}.69 \\
.66 \\
.51 \\
.55 \\
.45\end{array}$ & $\begin{array}{l}4.0 \\
4.0 \\
5.2 \\
4.6 \\
5.0\end{array}$ & $\begin{array}{l}3.27 \\
3.42 \\
3.04 \\
3.18 \\
3.49\end{array}$ & $\begin{array}{l}25.4 \\
25.0 \\
32.3 \\
29.8 \\
32.8\end{array}$ & $\begin{array}{l}166 \\
163 \\
221 \\
210 \\
220\end{array}$ & $\begin{array}{l}18.9 \\
18.4 \\
15.2 \\
17.7 \\
17.0\end{array}$ & $\begin{array}{r}98.0 \\
99.0 \\
125.0 \\
113.0 \\
121.0\end{array}$ & $\begin{array}{l}316 \\
303 \\
401 \\
380 \\
406\end{array}$ & $\begin{array}{l}6.4 \\
6.0 \\
5.1 \\
6.1 \\
6.2\end{array}$ \\
\hline $\begin{array}{l}D-1 \ldots \ldots . \\
D-2 \ldots \ldots . \\
D-3 \ldots \ldots \\
D-4 \ldots \ldots \\
E-4 \ldots \ldots\end{array}$ & $\begin{array}{l}.53 \\
.32 \\
.42 \\
.53 \\
.40\end{array}$ & $\begin{array}{l}.63 \\
.39 \\
.50 \\
.64 \\
.48\end{array}$ & $\begin{array}{l}3.2 \\
4.8 \\
5.6 \\
4.0 \\
4.5\end{array}$ & $\begin{array}{l}4.25 \\
2.37 \\
2.84 \\
2.49 \\
5.30\end{array}$ & $\begin{array}{l}20.6 \\
29.3 \\
37.2 \\
25.0 \\
28.8\end{array}$ & $\begin{array}{l}130 \\
192 \\
136 \\
151 \\
156\end{array}$ & $\begin{array}{l}19.8 \\
14.1 \\
11.8 \\
19.0 \\
14.6\end{array}$ & $\begin{array}{r}66.9 \\
120.0 \\
141.0 \\
96.0 \\
\ldots \ldots . .\end{array}$ & $\begin{array}{l}206 \\
302 \\
248 \\
252 \\
275\end{array}$ & $\begin{array}{r}5.5 \\
4.5 \\
3.6 \\
5.2 \\
\ldots . .\end{array}$ \\
\hline $\begin{array}{l}\mathbf{E}-6 \ldots \ldots \\
\mathbf{E}-7 \ldots \ldots \\
\mathbf{E}-8 \ldots \ldots \\
\mathbf{E}-9 \ldots \ldots \\
\mathbf{E}-10 \ldots \ldots\end{array}$ & $\begin{array}{l}.43 \\
.56 \\
.58 \\
.37 \\
.30\end{array}$ & $\begin{array}{l}.52 \\
.67 \\
.69 \\
.45 \\
.36\end{array}$ & $\begin{array}{l}4.0 \\
2.7 \\
2.0 \\
4.0 \\
5.8\end{array}$ & $\begin{array}{l}3.42 \\
3.20 \\
3.19 \\
3.28 \\
4.88\end{array}$ & $\begin{array}{l}25.4 \\
17.1 \\
31.3 \\
27.8 \\
36.2\end{array}$ & $\begin{array}{l}254 \\
187 \\
150 \\
149 \\
220\end{array}$ & $\begin{array}{r}24.0 \\
25.5 \\
27.0 \\
9.4 \\
18.3\end{array}$ & $\begin{array}{r}99.0 \\
65.5 \\
48.9 \\
\ldots \ldots . . \\
142.0\end{array}$ & $\begin{array}{l}446 \\
345 \\
262 \\
300 \\
396\end{array}$ & $\begin{array}{r}8.2 \\
9.6 \\
9.5 \\
\hdashline . .5\end{array}$ \\
\hline $\begin{array}{l}\mathrm{G}-1 \ldots \ldots \ldots \\
\mathrm{G}-2 \ldots \ldots \ldots \\
\mathrm{G}-3 \ldots \ldots \\
\mathrm{G}-4 \ldots \ldots\end{array}$ & $\begin{array}{l}.37 \\
.52 \\
.38\end{array}$ & $\begin{array}{l}.45 \\
.63 \\
.45\end{array}$ & $\begin{array}{l}6.3 \\
5.1 \\
5.9 \\
5.6\end{array}$ & $\begin{array}{l}4.46 \\
4.45 \\
2.11\end{array}$ & $\begin{array}{l}40.1 \\
32.8 \\
36.2 \\
37.1\end{array}$ & $\begin{array}{r}95 \\
145 \\
200 \\
160\end{array}$ & $\begin{array}{r}8.6 \\
16.9 \\
18.0 \\
13.0\end{array}$ & $\begin{array}{l}\dddot{125} \\
138.0 \\
129.0\end{array}$ & $\begin{array}{l}147 \\
228 \\
336 \\
273\end{array}$ & $\begin{array}{l}2.1 \\
3.8 \\
4.9 \\
4.1\end{array}$ \\
\hline
\end{tabular}



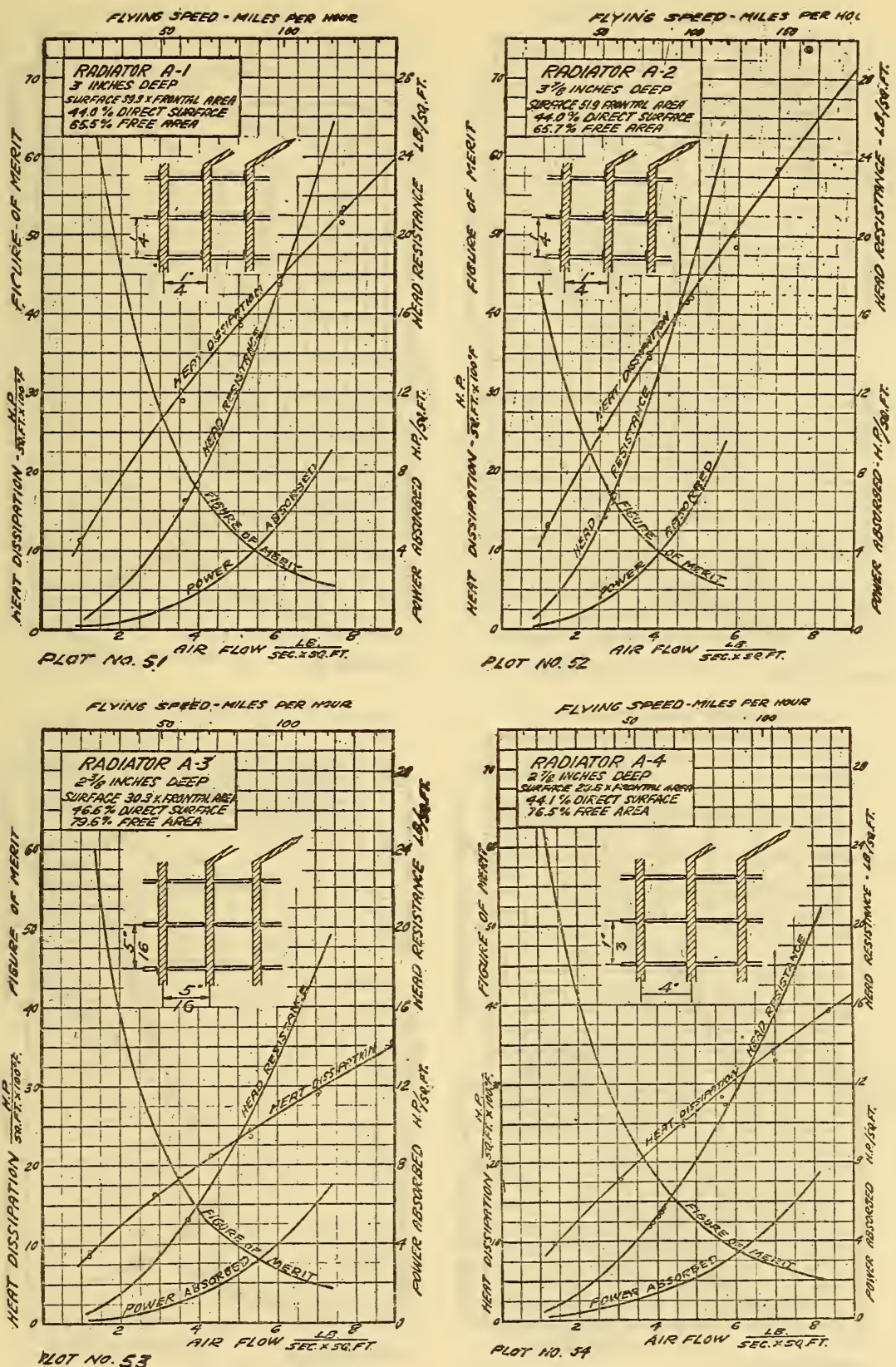

FIGS. 5I-54.-Performance curves of square cell radiator cores

Experimental data for these cores are summarized in Tables 20 and 21 . Units and definitions will be found in Arts. 2, 3, 4 

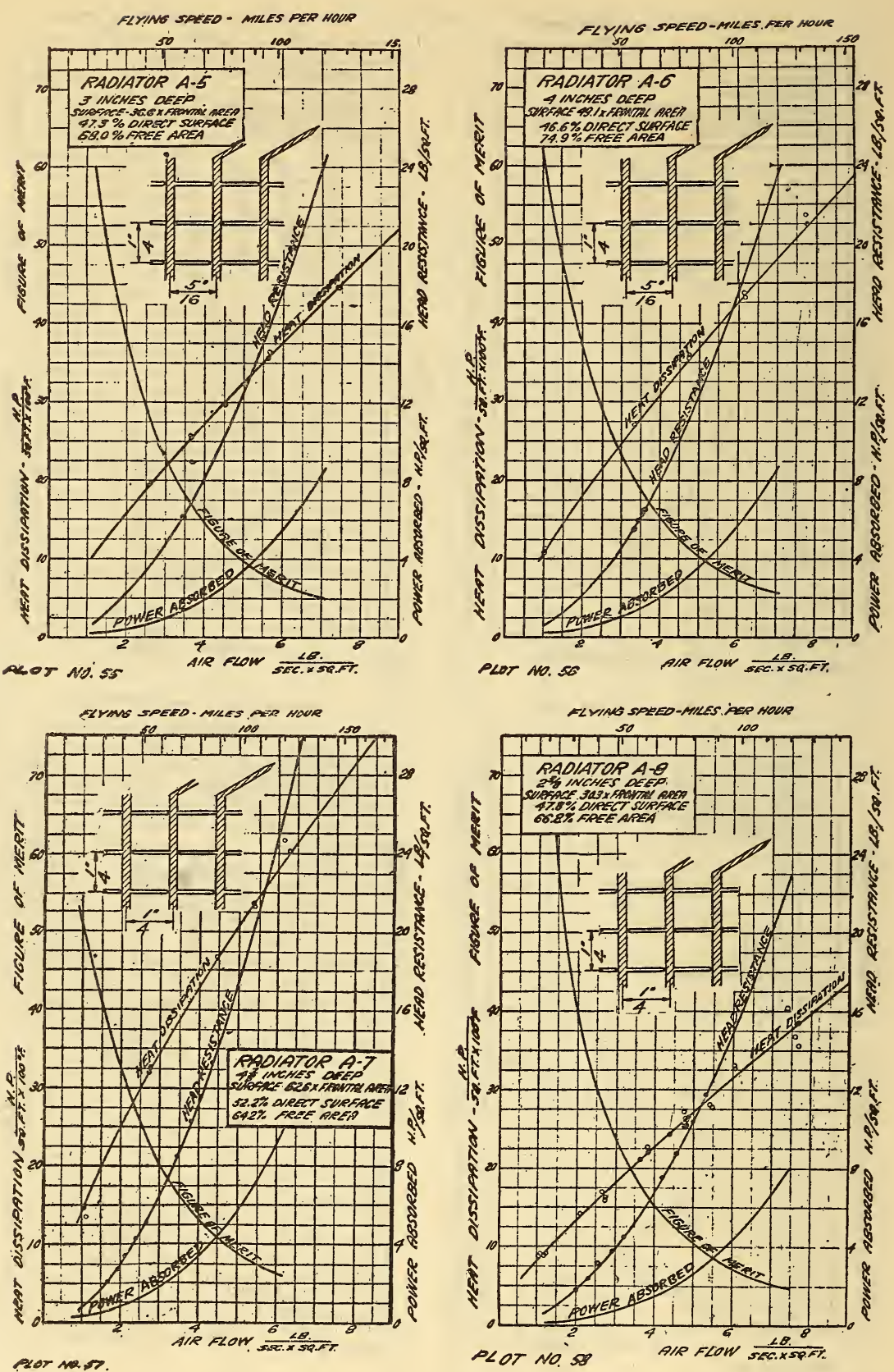

FIGs. 55-58.-Performance curves of square cell radiator cores

Experimental data for these cores are summarized in Tables 20 and $2 x$. Units and definitions will be found in Arts. 2, 3, 4 

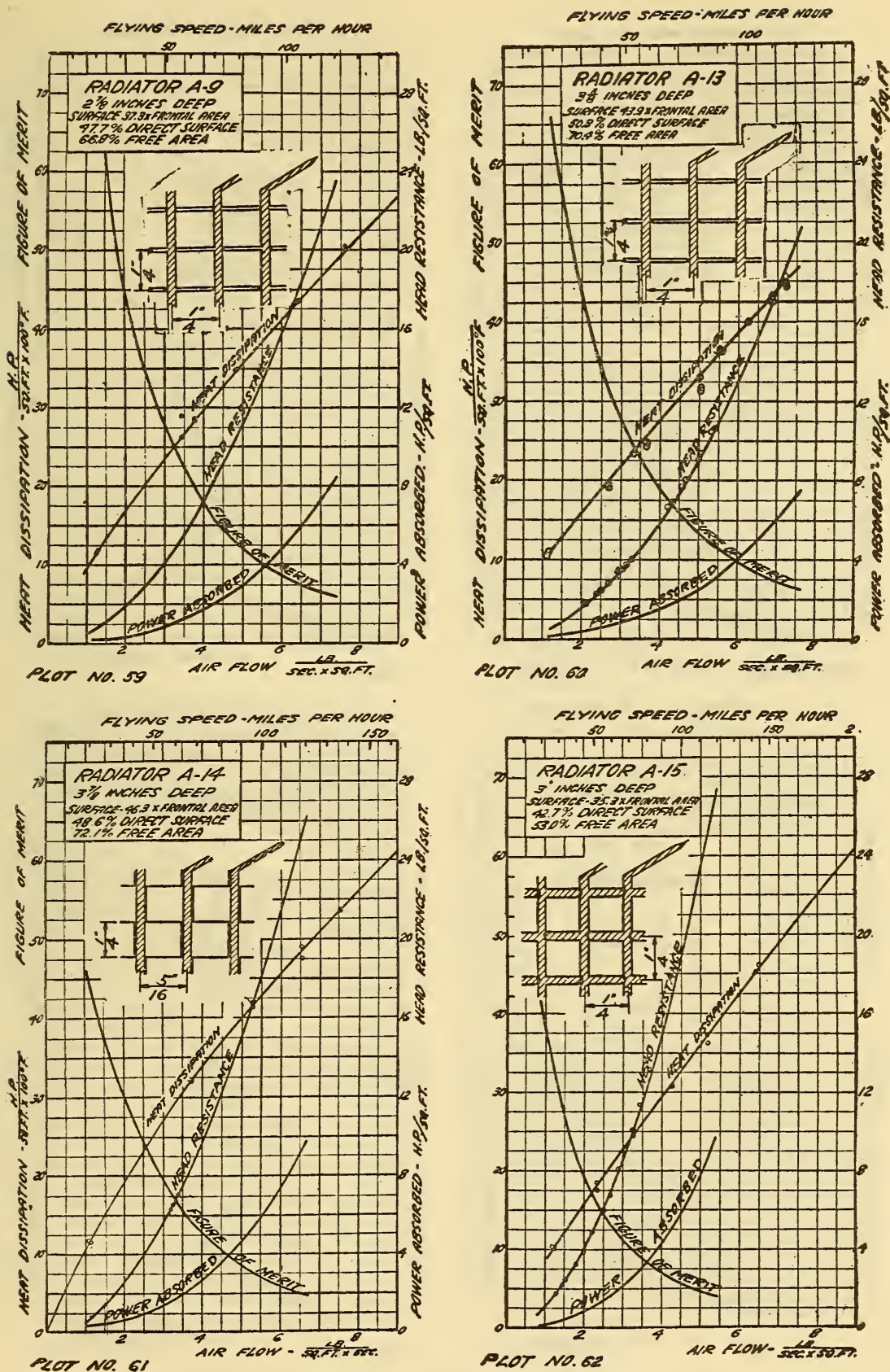

FIGS. 59-62.-Performance curves of square cell radiator cores

Experimental data for these cores are summarized in Tables 20 and $2 \mathrm{r}$. Units and definitions will be found in Arts. 2, 3, 4 

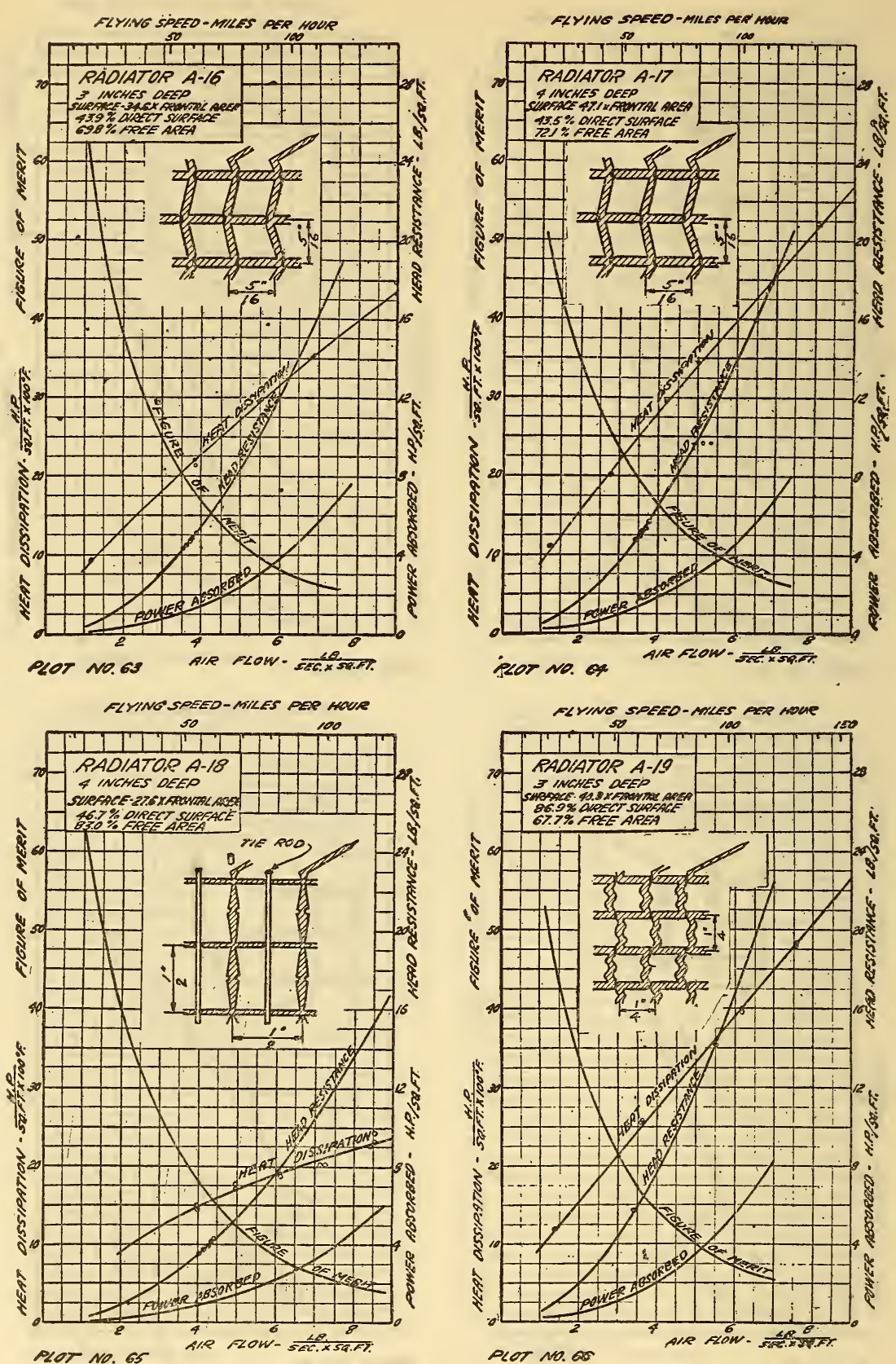

Figs. 63-66.-Performance curves of radiator cores with cells approximately rectangular

Experimental data for these cores are summarized in Tables 20 and $2 x$. Units and definitions will be found in Arts. 2, 3, 4 

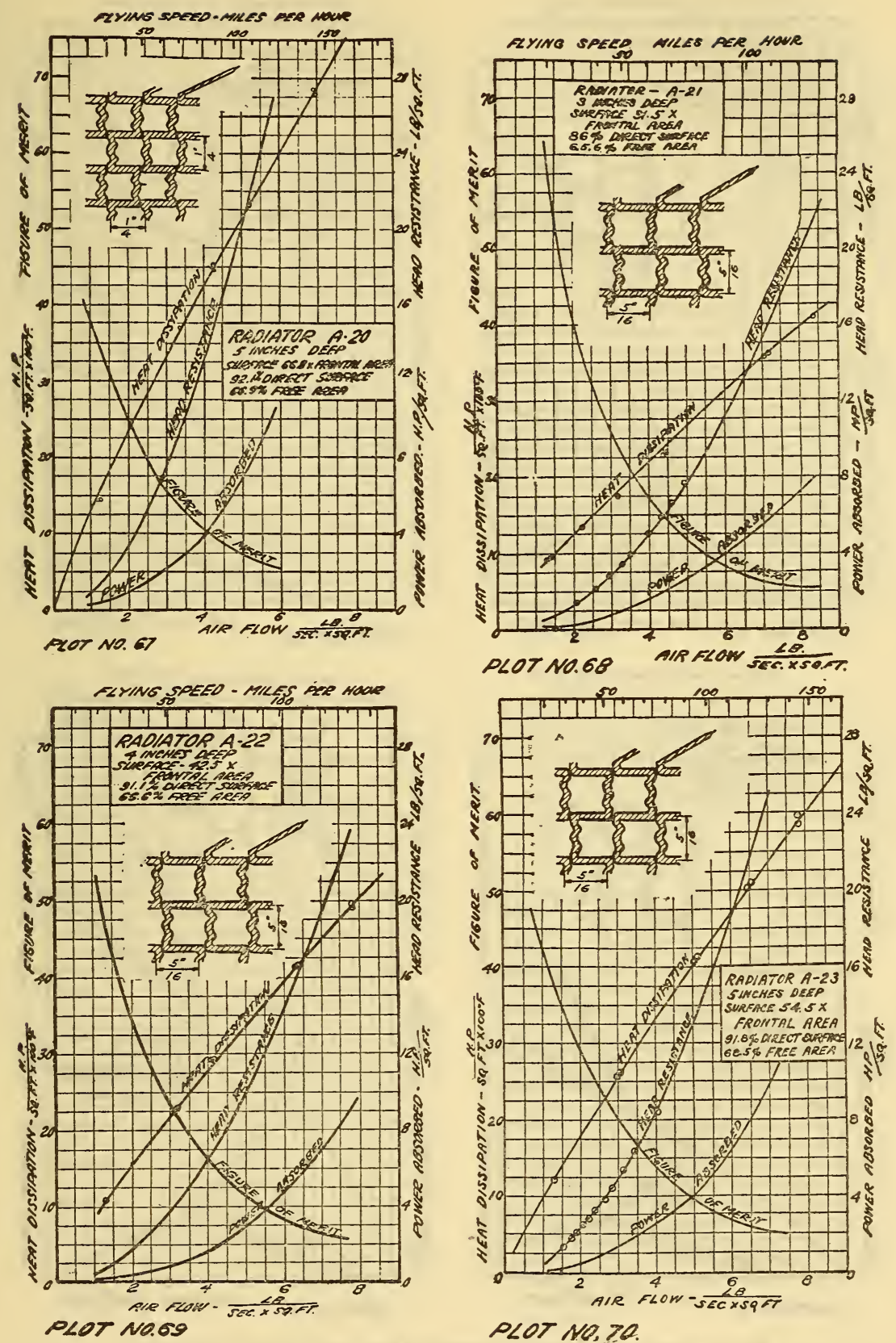

Figs. 67-70.-Performance curves of square cell radiator cores

Experimental data for these cores are summarized in Tables 20 and $2 r$. Units and definitions may be iound in Arts. 2, 3, 4 

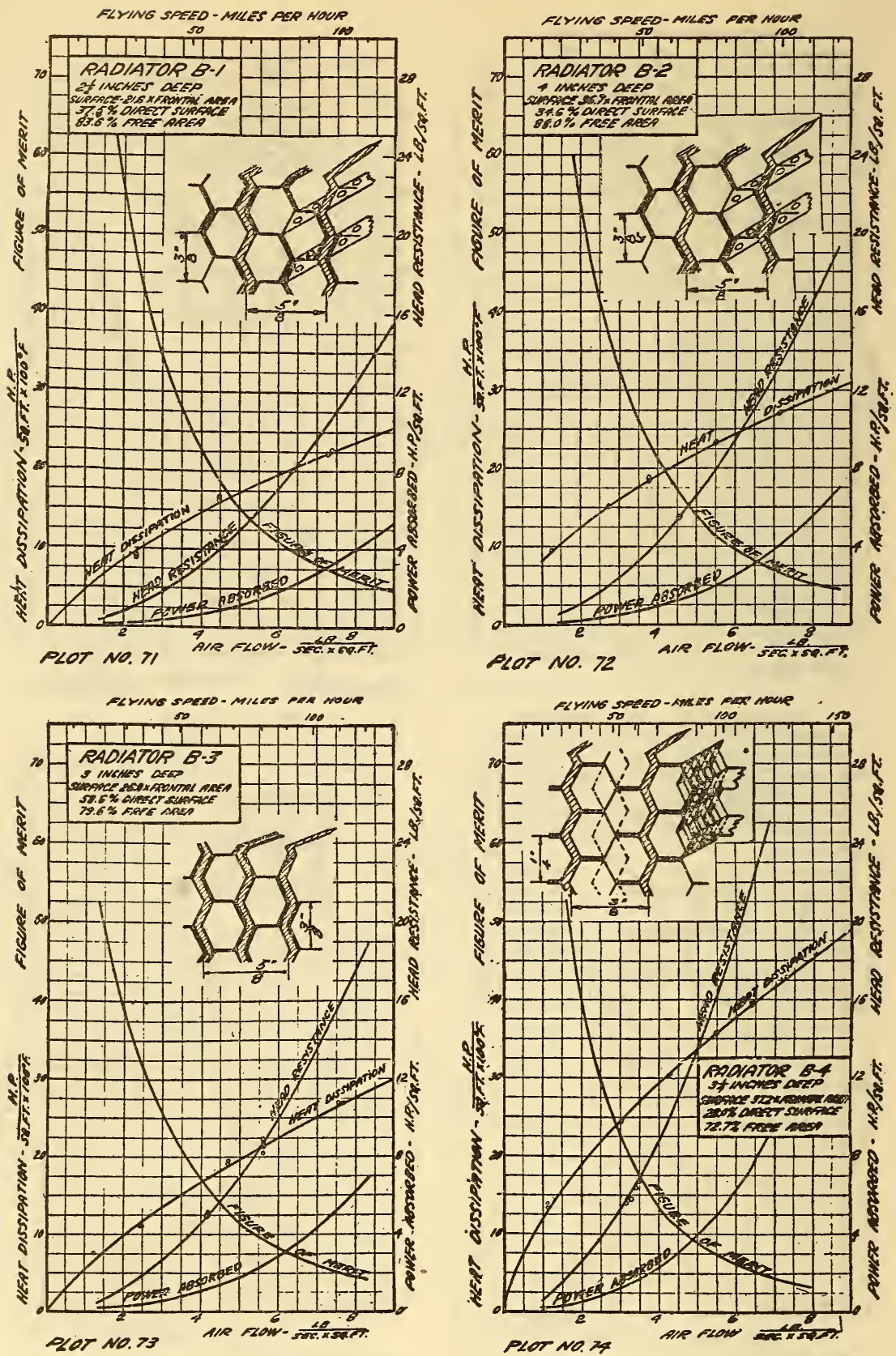

FIGS. 7I-74.-Performance curves of hexagonal cell radiator cores

Holes interconnecting the cells in three of the above four cores modify the air flow from that for a simple honeycomb of the same size. Experimental data for these cores are summarized in Tables 20 and 2I. Units and definitions may be found in Arts. 2, 3, 4 

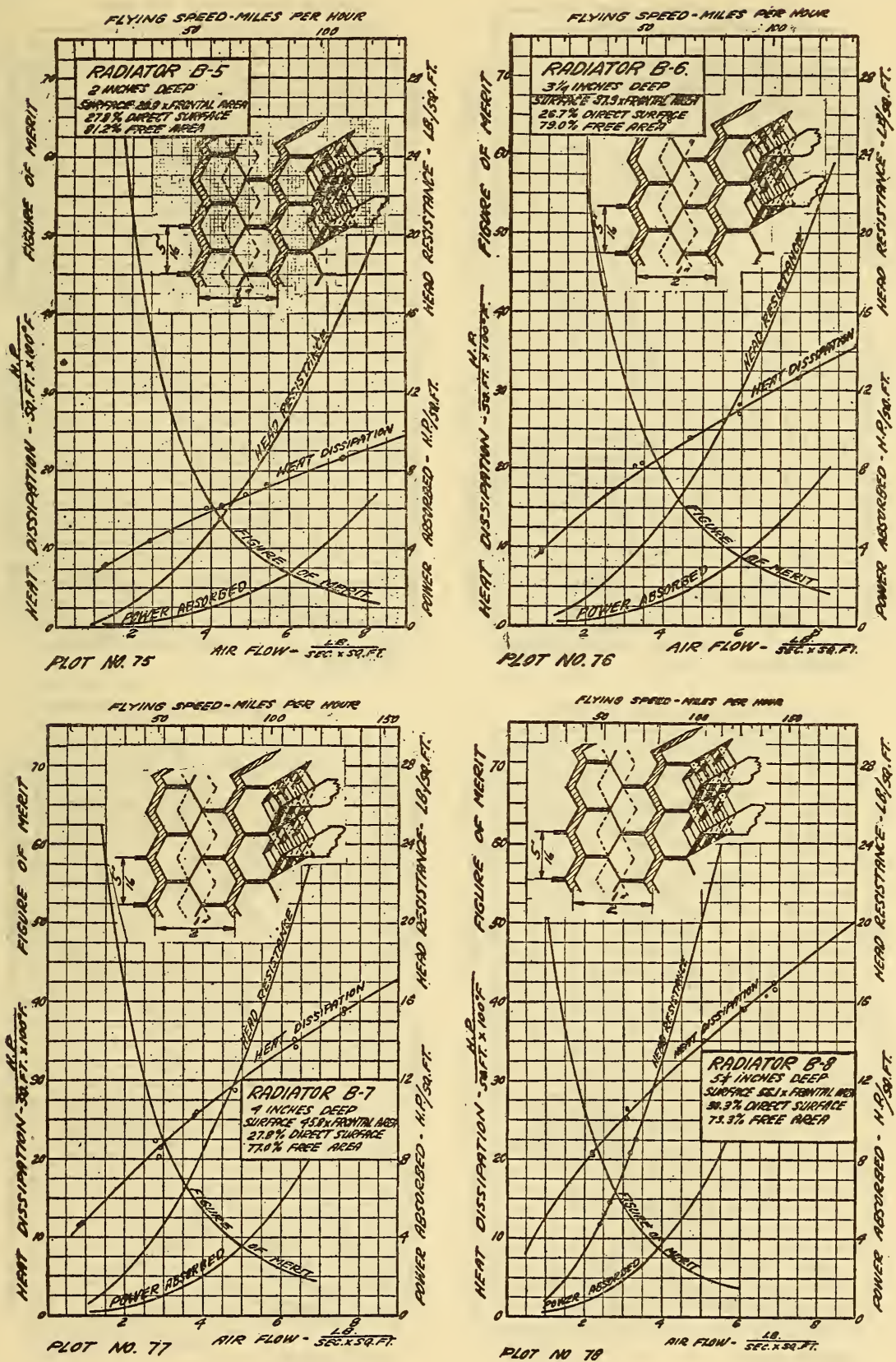

FIGs. 75-78.-Performance curves of hexagonal cell radiator cores similar in construction to those of Figs. 7I-74 except as to depth of core

The vertical walls which are not water tubes are perforated and latticed so as to modify the air flow considerably from that in a plain hexagonal honeycomb. Experimental data for these cores are summarized in Tables 20 and 21 . Units and definitions will be found in Arts. 2, 3, 4 

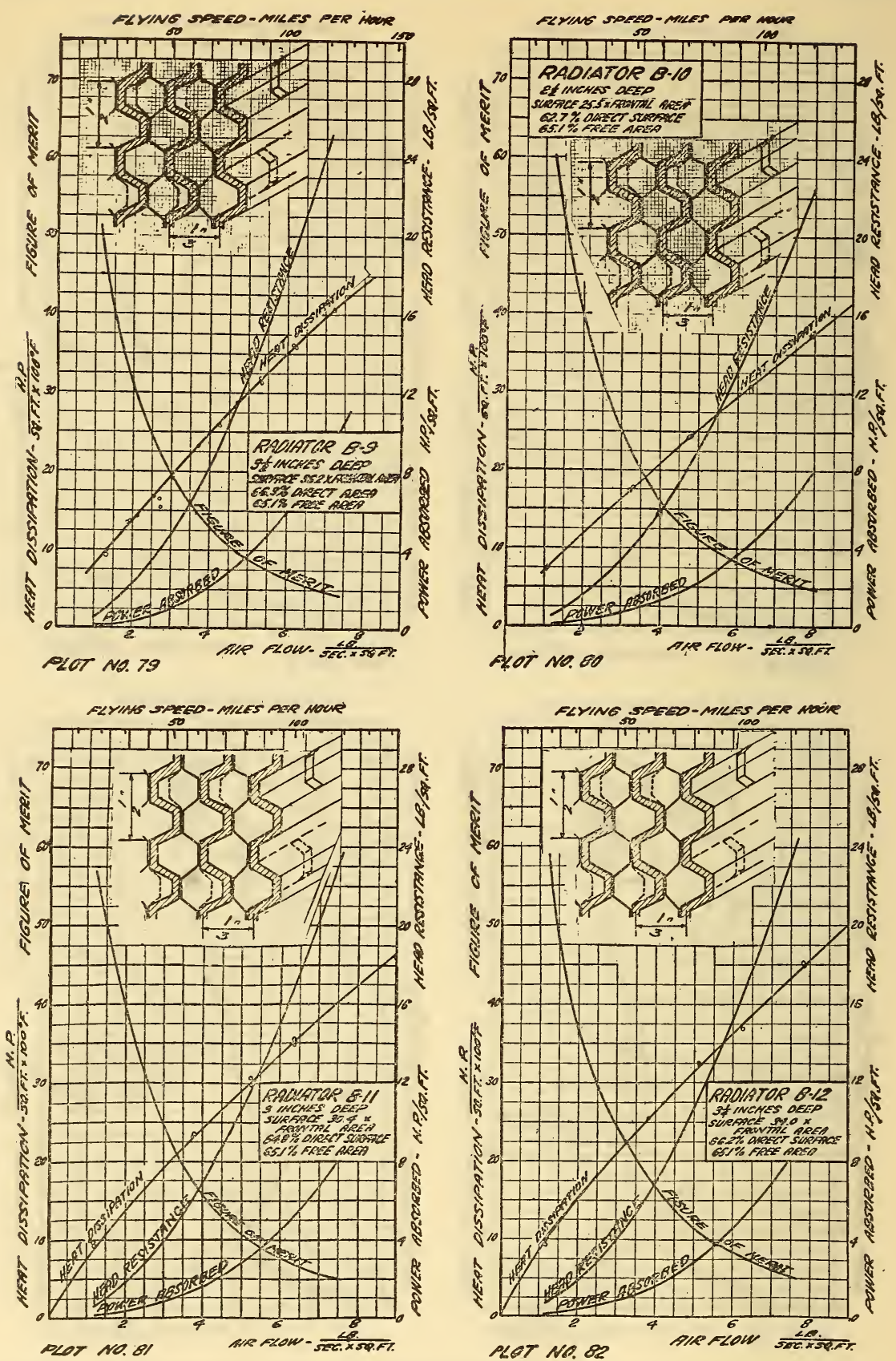

FIGS. 79-82.-Performance curves of hexagonal cell radiator cores, similar in construction to those of Figs. 7I-74, except as to depth of core

Unlike the cores of Figs. $75^{-78}$, the air tubes are independent, but small turbulence vanes are present. Experimental data for these cores are summarized in Tables 20 and 21 . Units and definitions will be found in Arts. 2, 3, 4 

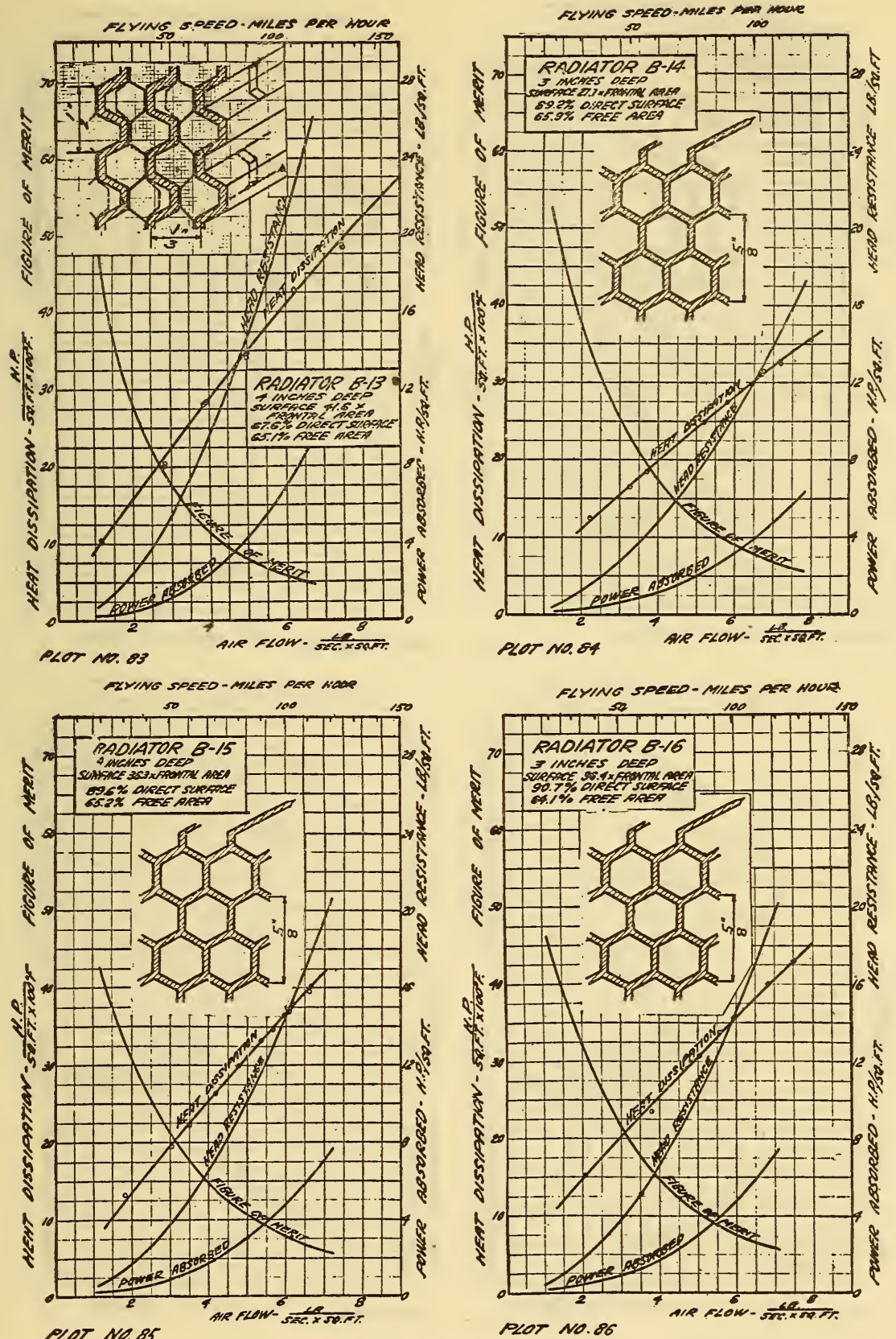

FIGS. 83-86.-Performance curves of hexagonal cell radiator cores

The core for Fig. $8_{3}$ is of construction identical with those shown in Figs. $79-82$, but deeper. The remaining three cores are of simple honeycomb construction without vanes. Experimental data for these cores are summarized in Tables 20 and 2I. Units and definitions will be found in Arts. 2, 3. 4 

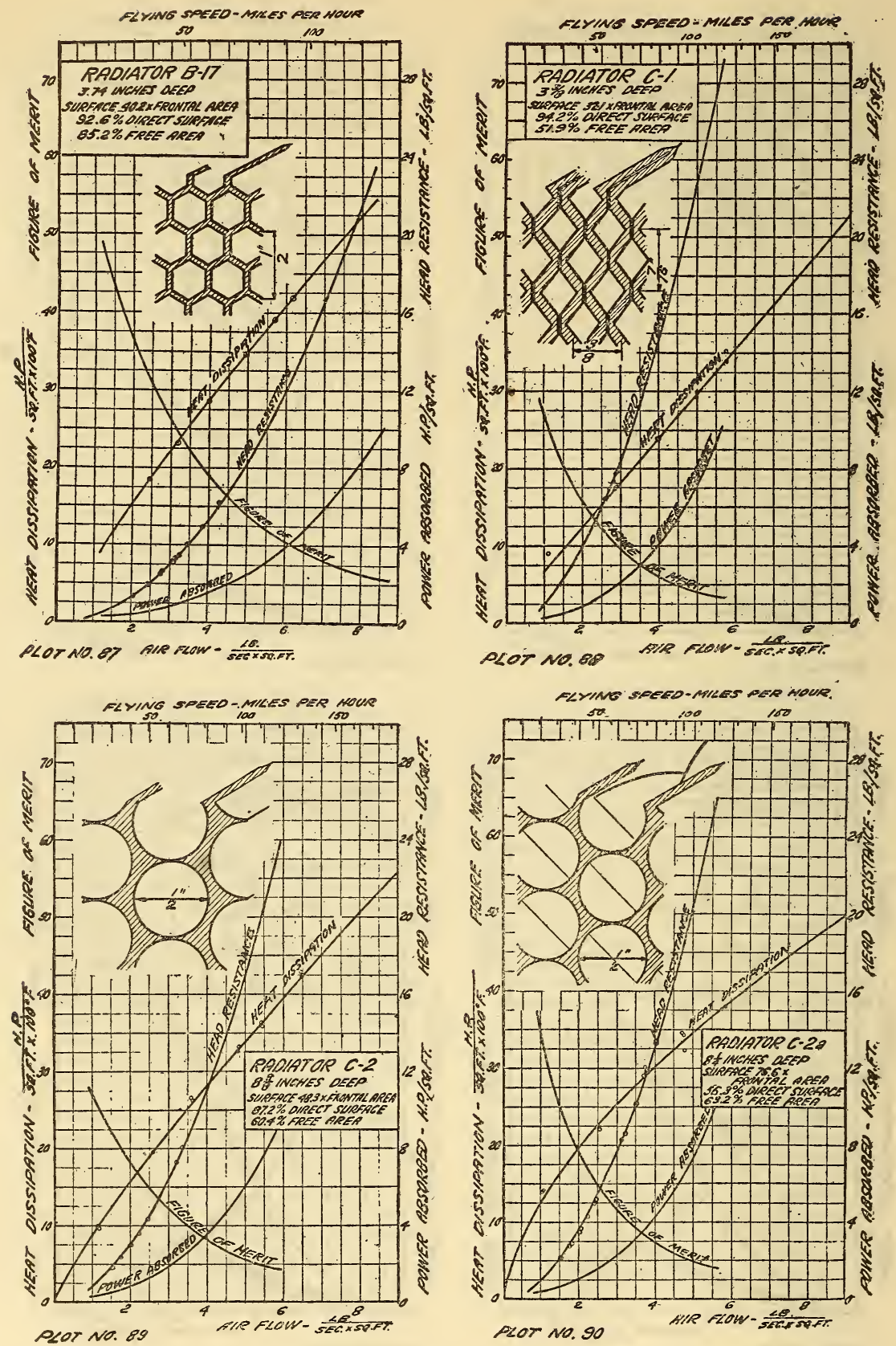

FIGs. 87-90.-Performance curves of cellular radiator cores

Cores $\mathrm{C}_{2}$ and $\mathrm{C}_{2 a}$ are identical except for a spiral turbulence vane in each air tube of $\mathrm{C}_{2}$. These reduce the air flow for given flying speed and as the curves show, figure of merit in an unobstructed position in terms of flying speed is nearly alike for both cores, the vanes introducing a slight advantage at low speeds and disadvantage at high speeds. Experimental data for these four cores are summarized in Tables 20 and 21. Units and definitions are given in Arts. 2, 3, 4. 

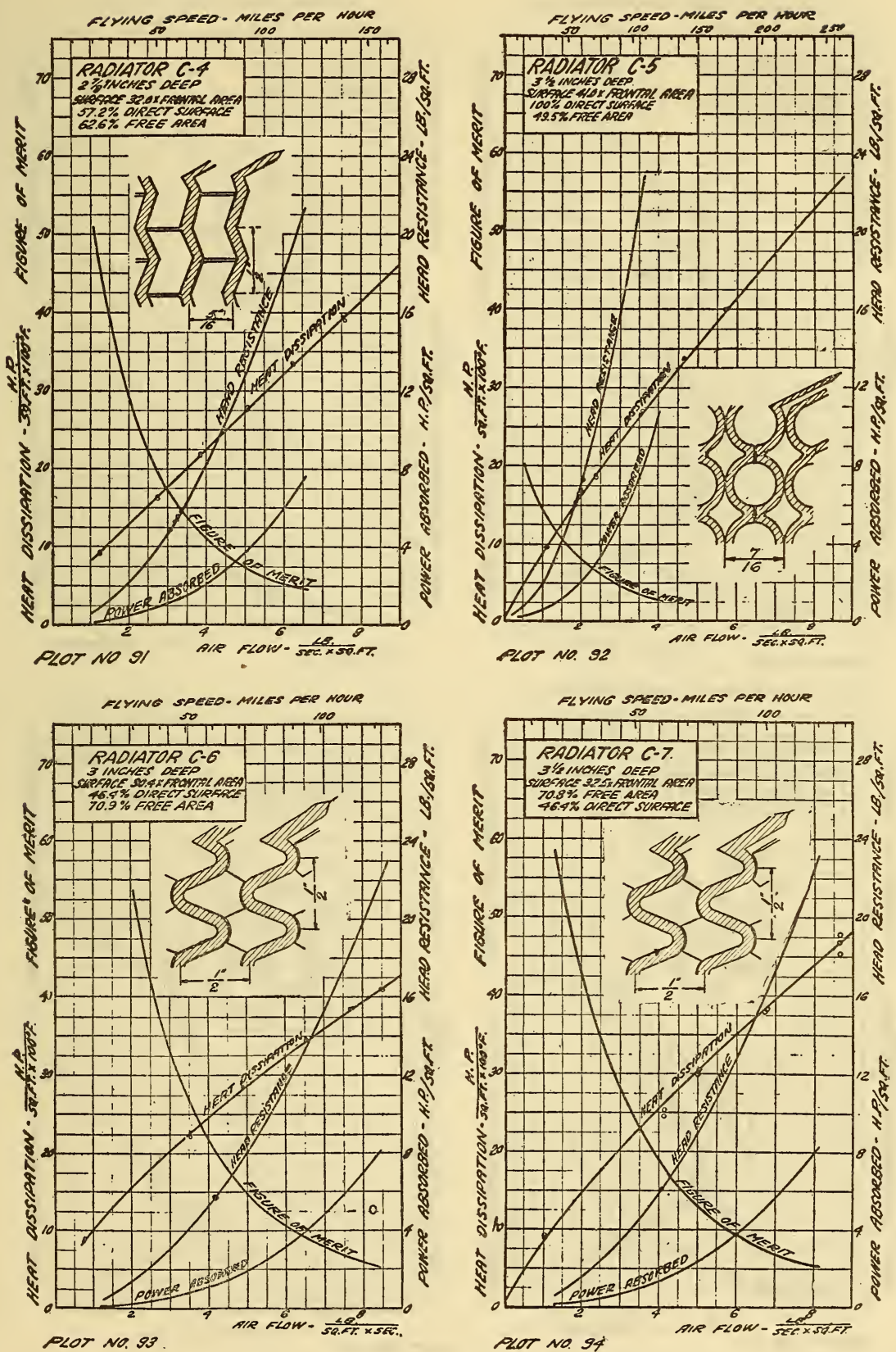

Fias. 91-94.-Performance curves of miscellaneous cellular radiator cores

Experimental data for these cores are summarized in Tables 20 and $2 \mathrm{I}$. Units and definitions may be found in Arts. 2, 3, 4 

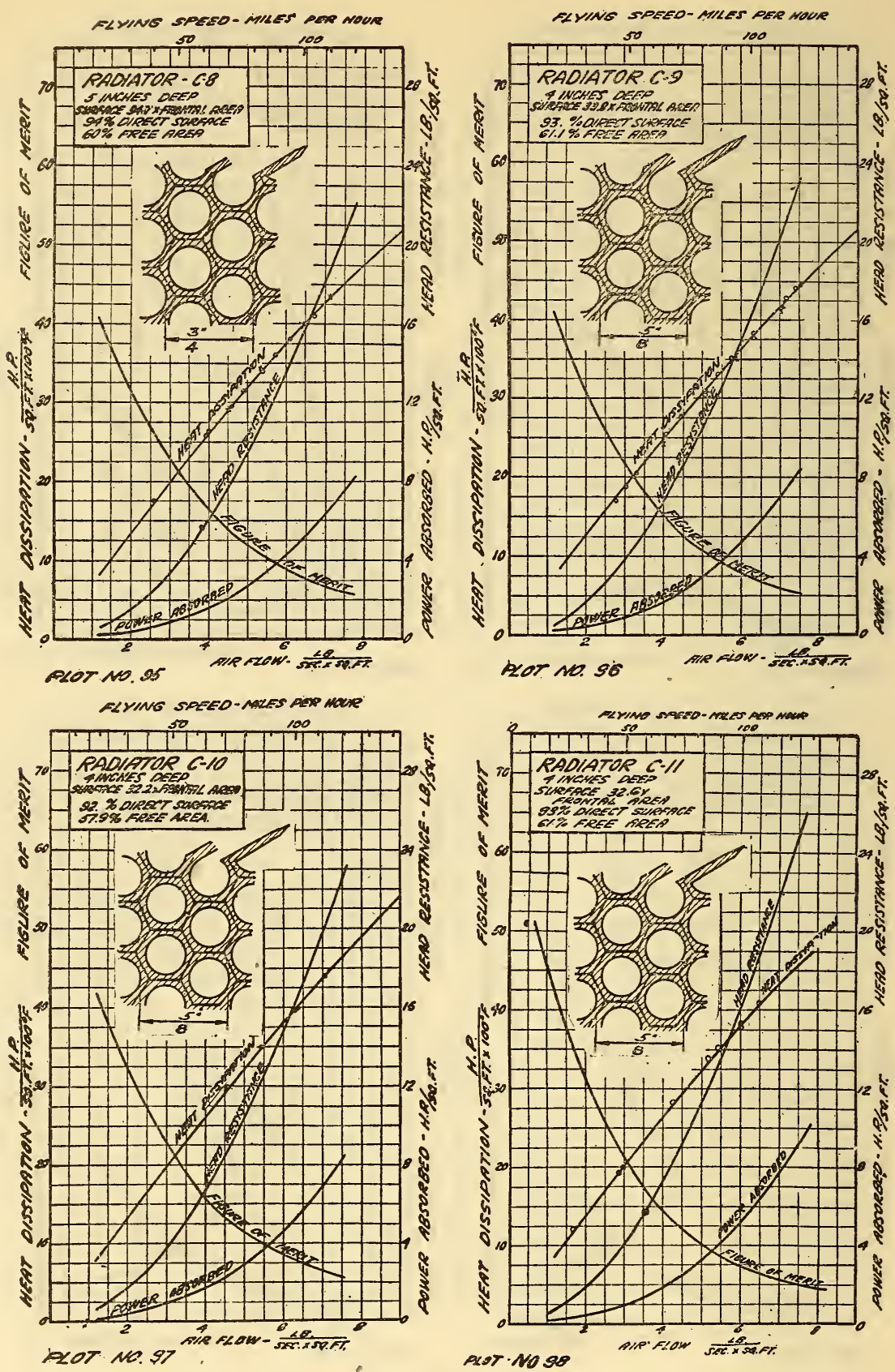

FIGs. 95-98. Performance curves of cellular radiator cores with circular air tubes, the ends of which are pressed into hexagons for joining at the core faces

Experimental data for these cores are summarized in Tables 20 and 21 . Units and definitions may be found in Arts. 2, 3, 4 

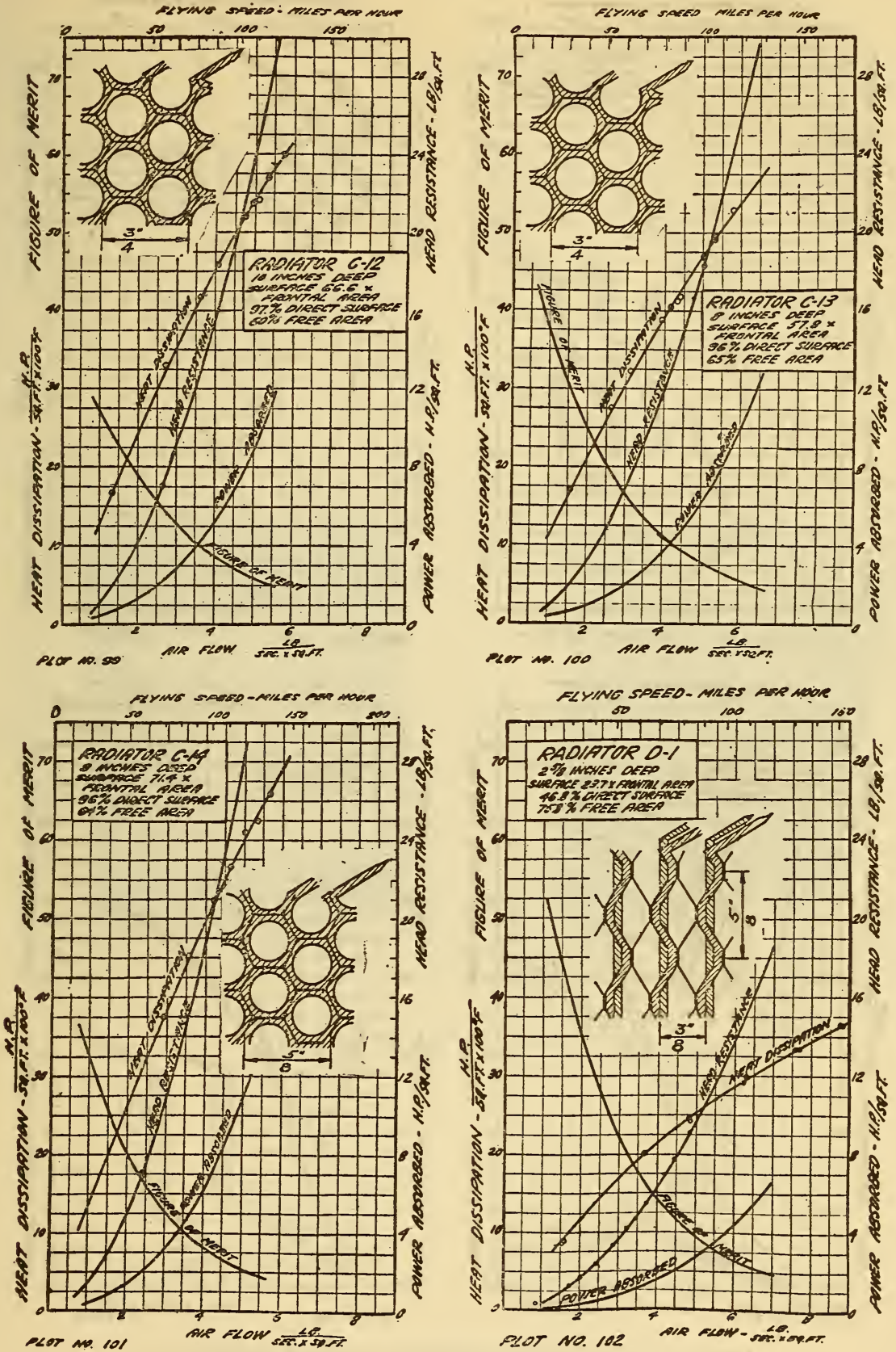

FIGS. 99-102.-Performance curves of cellular radiator cores, the circular tube cores with hexagonal ends being similar except in depth to either C-8 of Fig. 95 or C-9 of Fig. 96

The corrugated or dimpled form of water tube composing $D-I$ is not illustrated adequately by the section drawing and a photograph of the core forms Fig. 103. Experimental data for these cores are summarized in Tables 20 and $2 x$. Units and definitions may be found in Arts. 2, 3, 4 $75013^{\circ}-22-10$ 

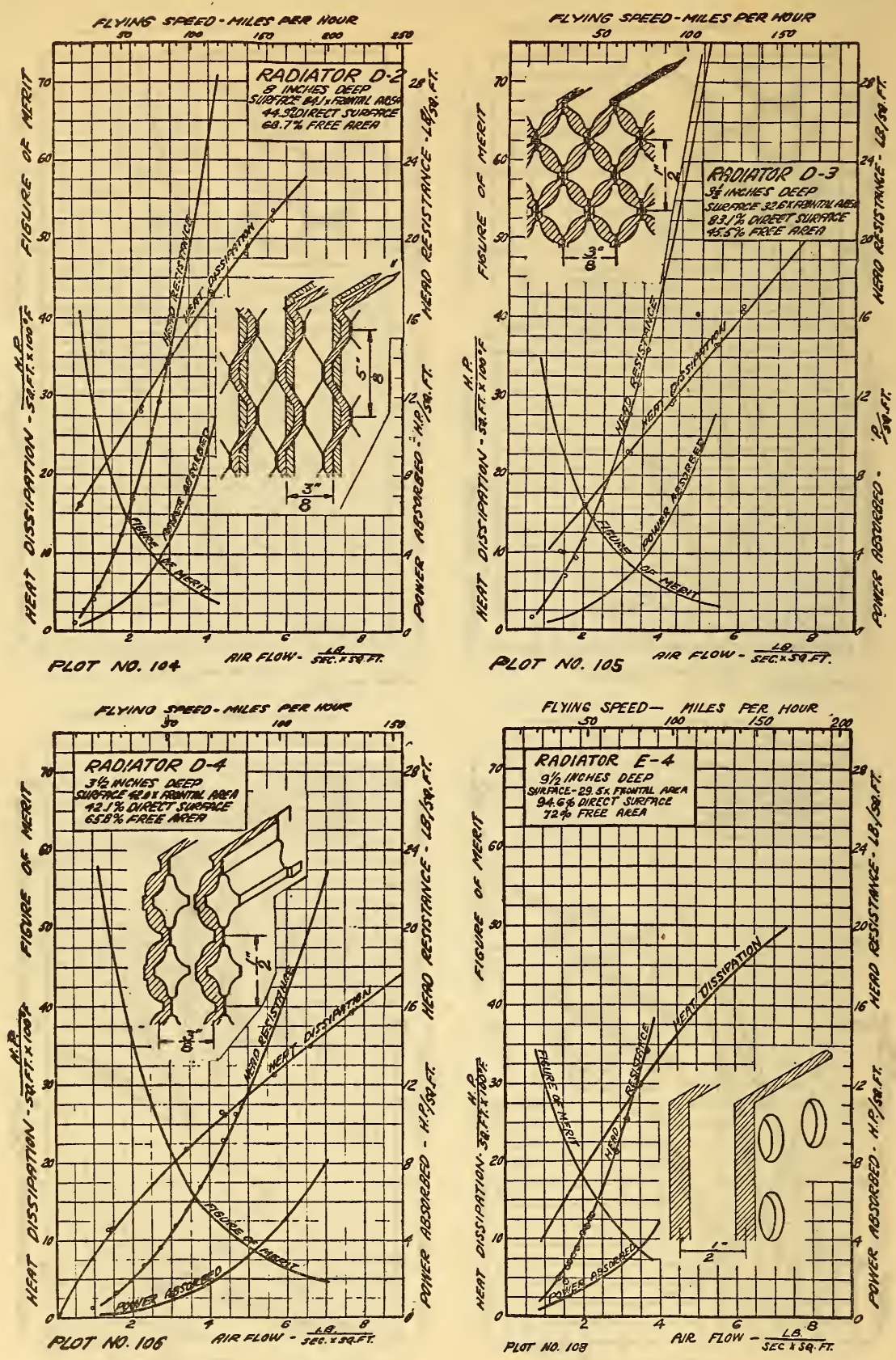

FIGS. 104-105-106-108.-Performance curves of radiator cores

Core D-2 (Fig. I04) is similar except in depth to D-r (Figs. 102, I03). Core E-4 is of flat plate construction, perforated with holes which cause it to whistle in an air stream. The construction is shown in Fig. I07, a photograph of a flat plate whistling radiator. Experimental data for these cores are sum-7 marized in Tables 20 and 2r. Units and definitions may be found in Arts. 2, 3, 4 
Technologic Papers of the Bureau of Standards, Vol. 16

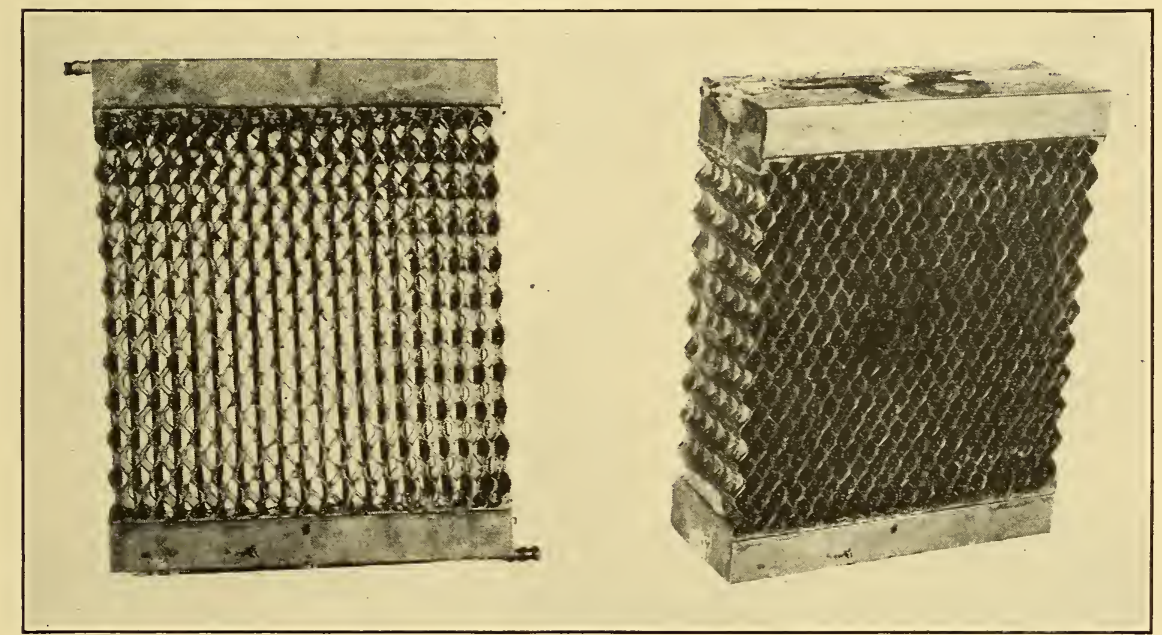

FIG. I03.-Core section D-I for which properties are shown in curves of Fig. IO2. Core D-2 (Fig. IO4) is similar construction, but deeper 
Technologic Papers of the Bureau of Standards, Vol. 16

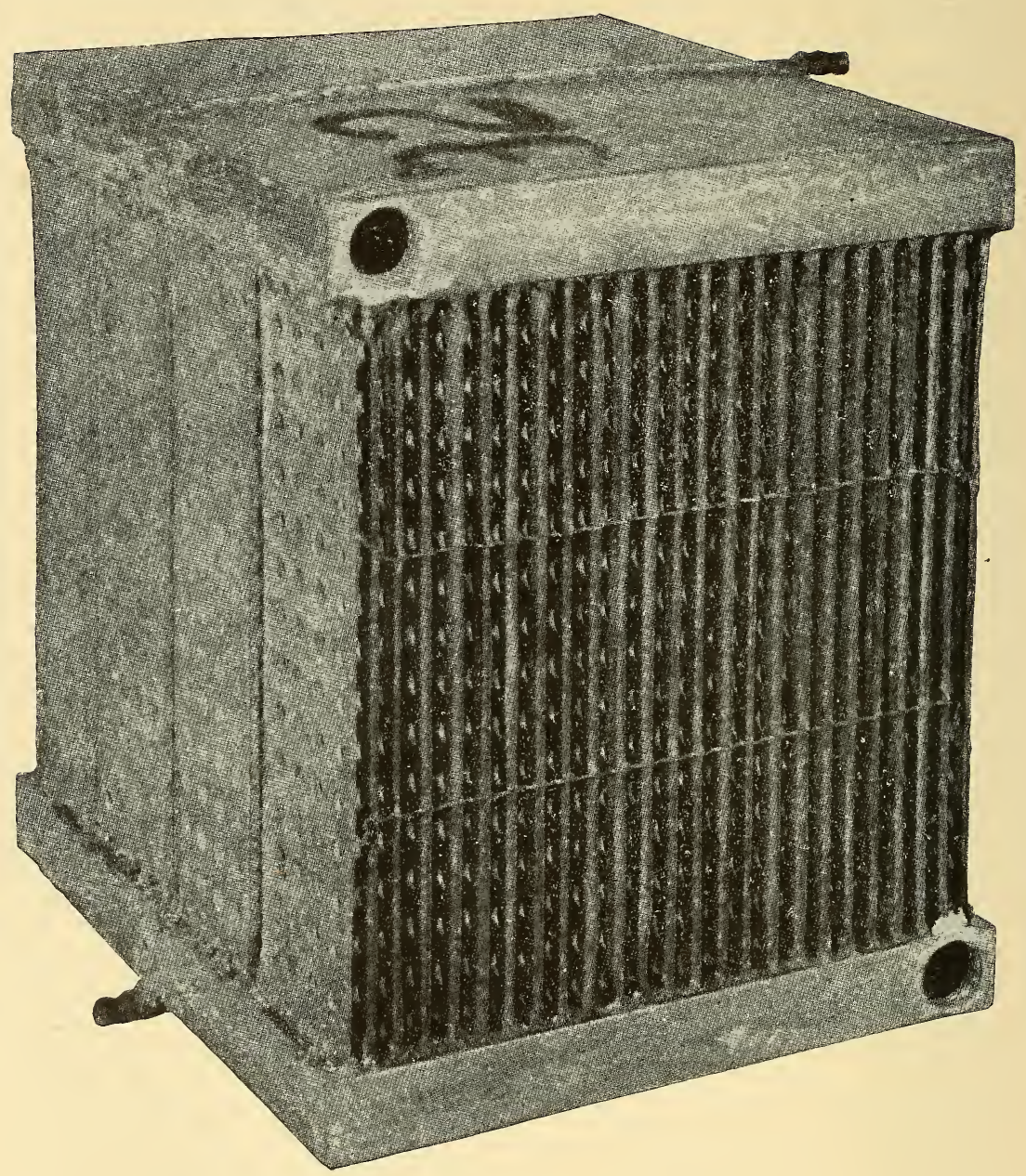

FIG. I07.-Flat plate core E-3 with perforations in plates causing the radiator to whistle

Cores $\mathrm{E}-\mathbf{1}, \mathrm{E}-2, \mathrm{E}-3$ have plates spaced $5 / 16$ in. apart, and cores $\mathrm{E}-4$ and $\mathrm{E}-5$ are similar in all respects except for the more open plate spacings of $\mathrm{I} / 2$ and $5 / 8$ in. The properties of $\mathrm{E}-4$ are shown in the curves of Fig. 108. 

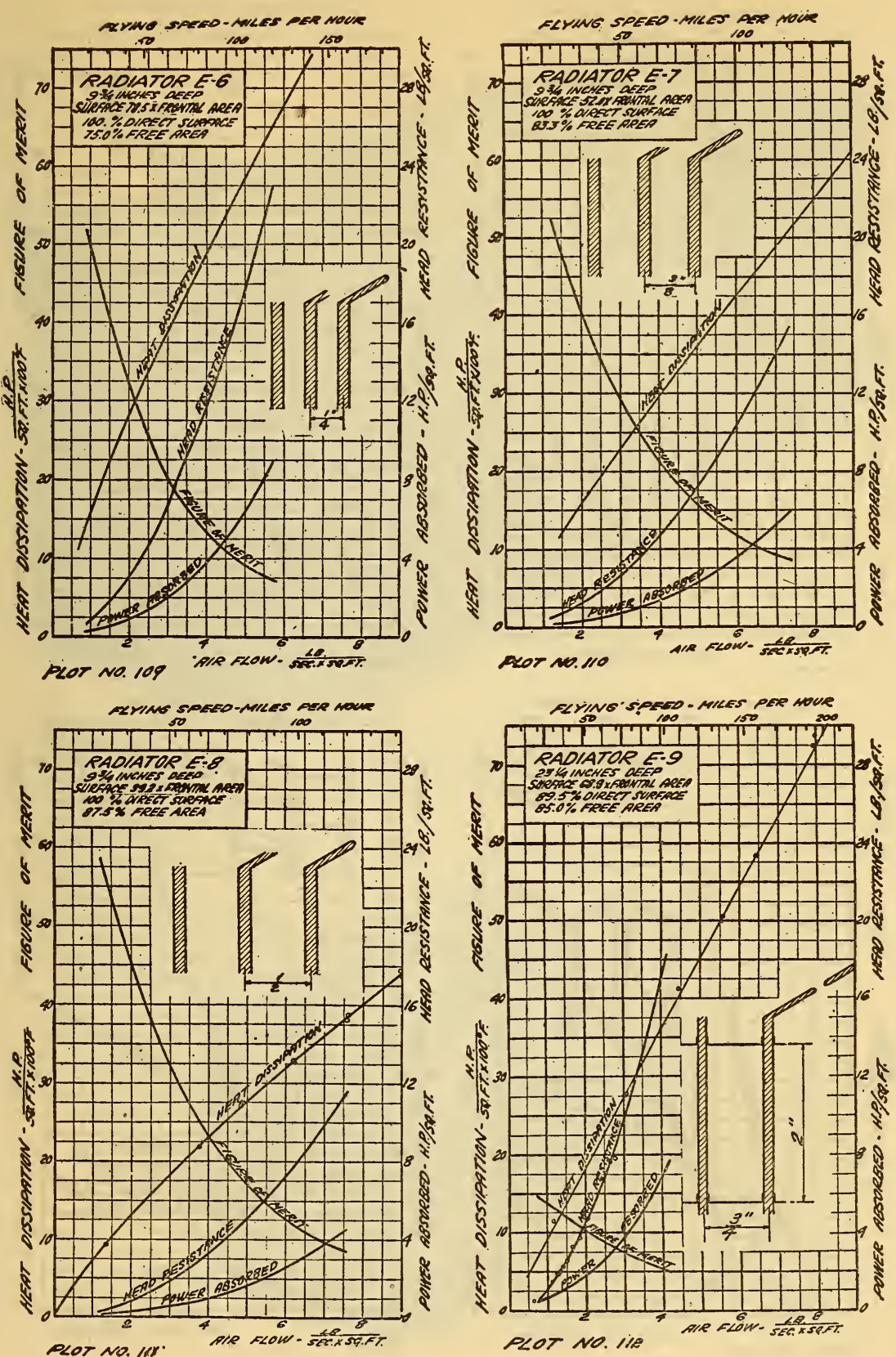

FIGS. Io9-II2.-Performance of flat plate radiator cores

This type of core shows the highest figure of merit at large fiying speeds, in an unobstructed position, of any type reported in this paper. Such disadvantages as are evident are chiefly mechanical-rigidity, ease of construction and repair, etc. The construction of E-9 (Fig. II2) is illustrated by a photograph of the test specimen, Fig. II3. Experimental data for these cores are summarized in Tables 20 and 2 I. Units and definitions may be found in Arts. 2, 3, 4 

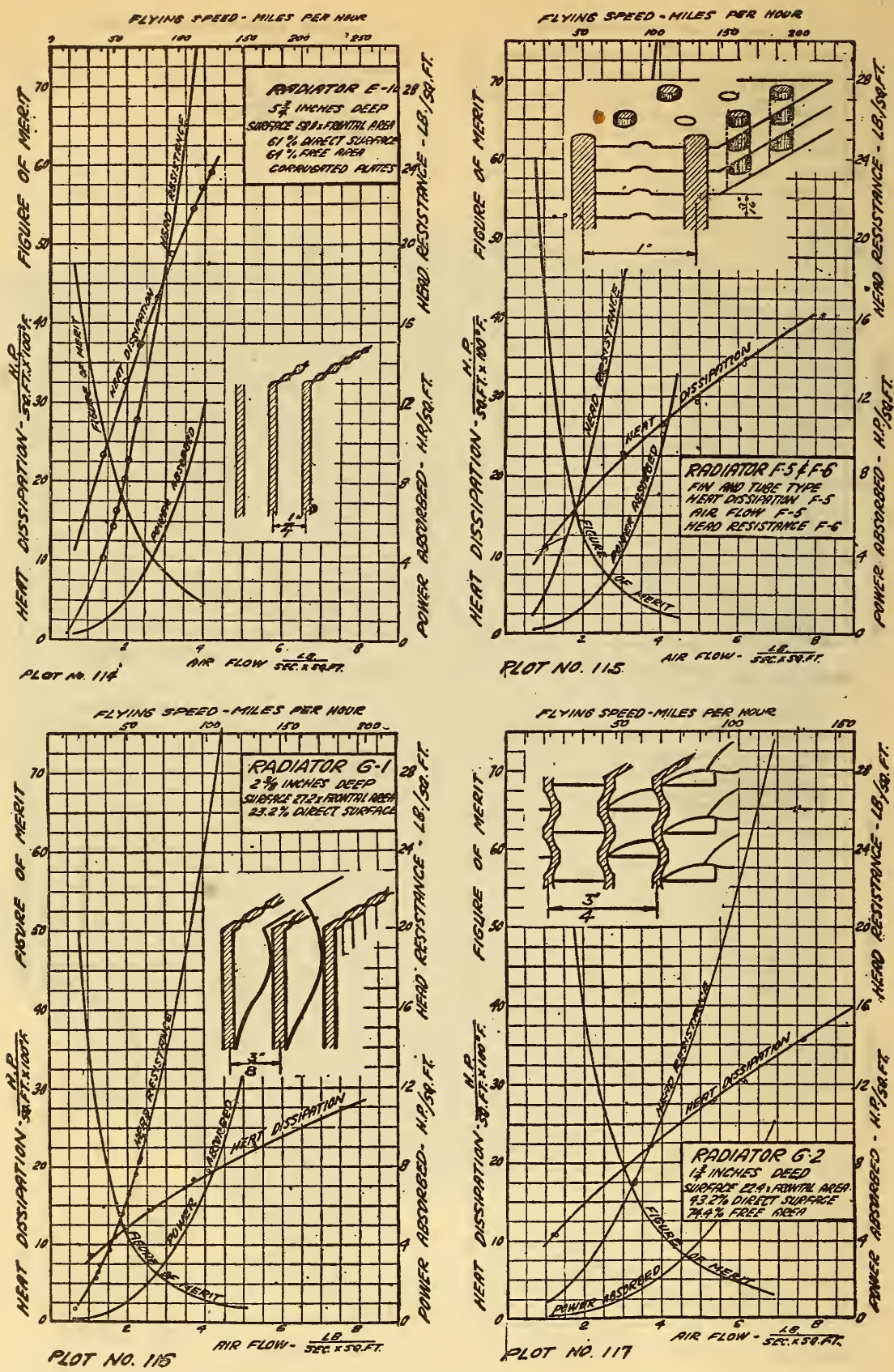

FIGs. II4-II7.-Performance curves of radiator cores

The fin and tube type (Fig. Ir5), a construction which has been common in automobile use, has a figure of merit so low at large flying speeds as to discourage its use in aviation. The spiral vane construction of core $\mathrm{G}-2$ (Fig. II 7 ) is not illustrated adequately by the section drawing, and a photograph of a similar construction $(\mathrm{G}-3)$ is given in Fig. Ir8. Experimental data for these cores are summarized in Tables 20 and 2 r. Units and definitions may be found in Arts. 2, 3, 4 
Technologic Papers of the Bureau of Standards, Vol. 16

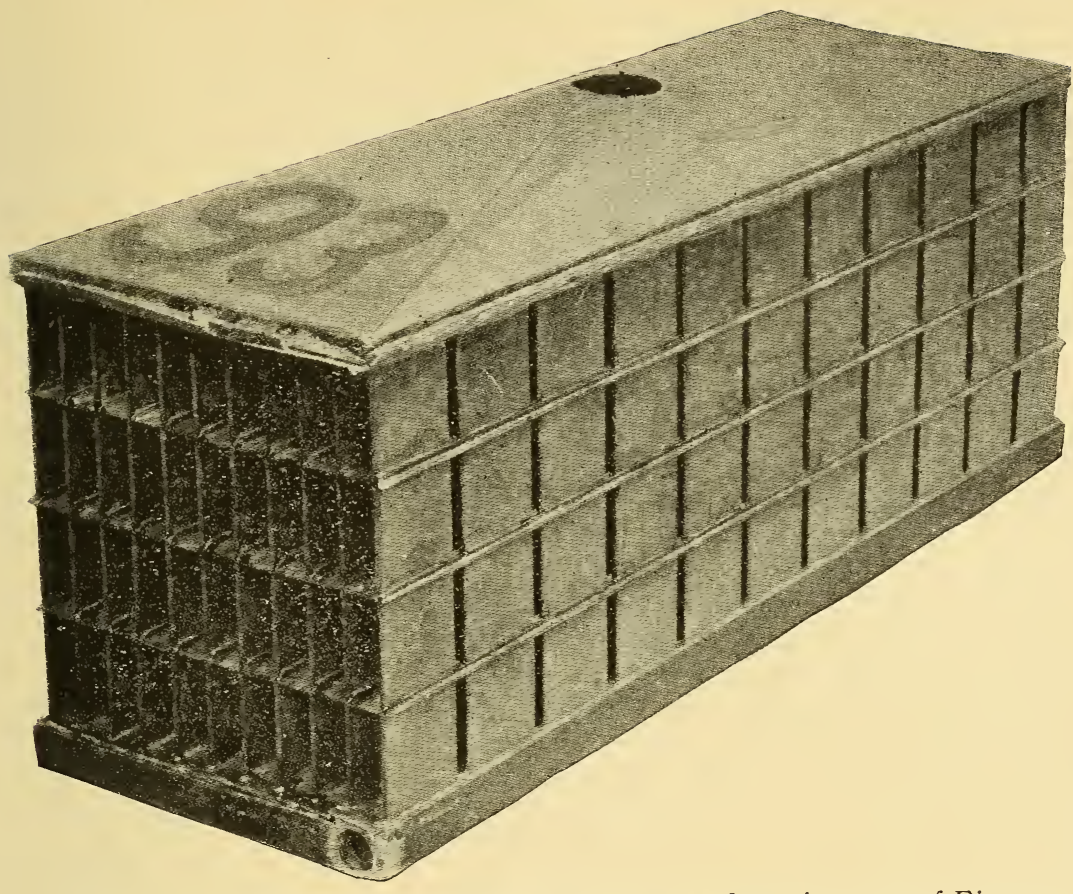

FIG. II3.-Core section E-g for which properties are shown in curves of Fig. II2

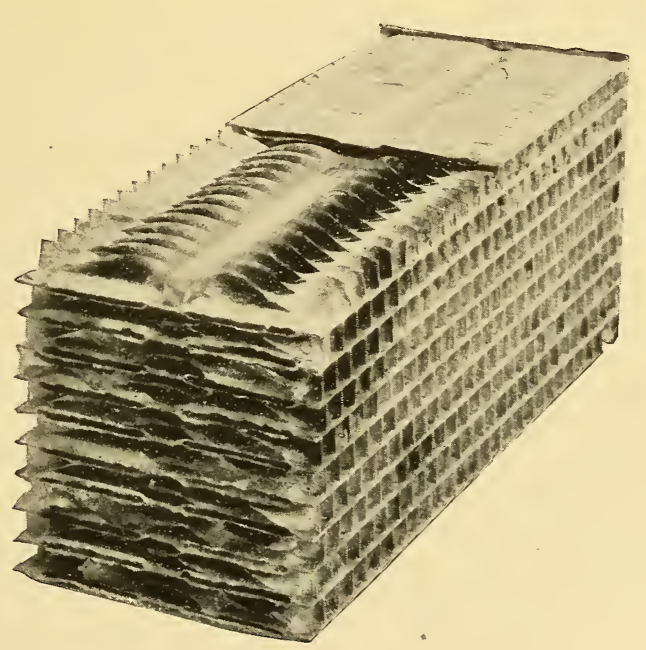

FIG. II8.-Spirex core section $G-3$ for which properties are shown in curves of Fig. IIg

Section G-2 (Fig. II ) exactly similar in construction, half as deep, has but one of the two sets of spiral vanes shown above 
Technologic Papers of the Bureau of Standards, Vol. 16
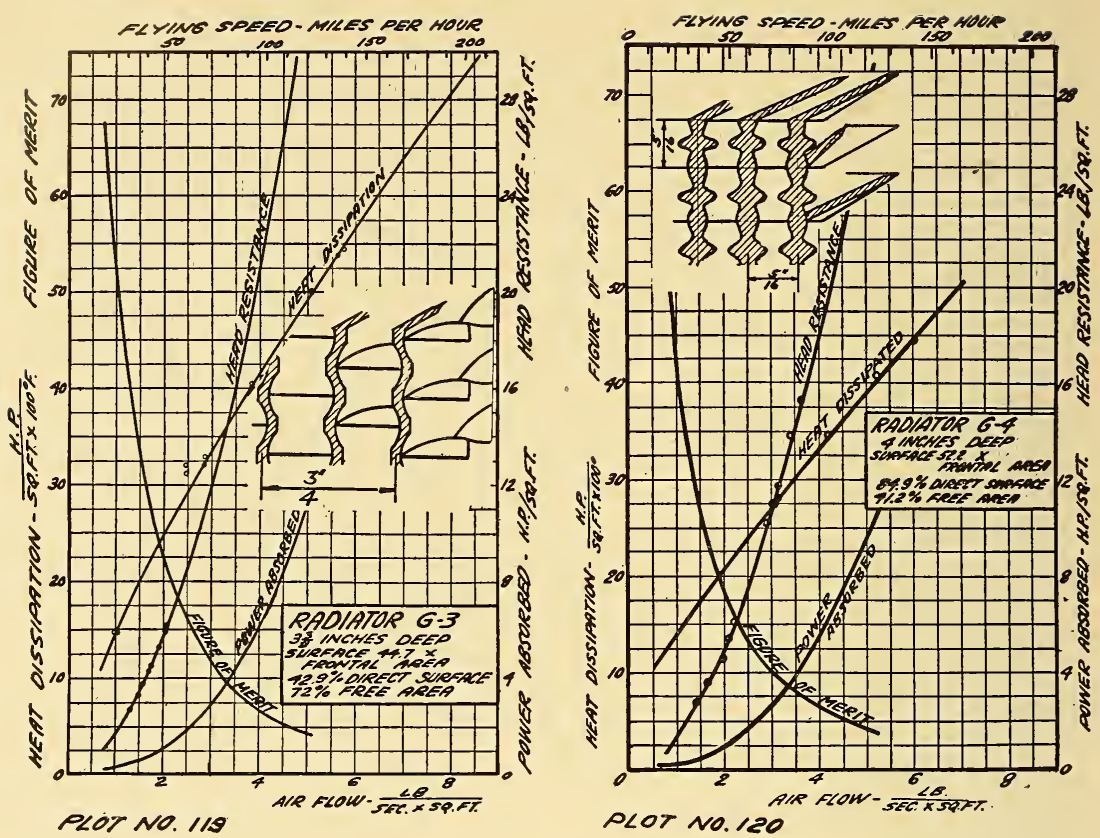

FIGS. II9-I20.-Performance curves of a spirex and a staggered cellular radiator core

The construction of the former is shown by a photograph in Fig. rr8, and of the latter in Fig. I2r. Experimental data for both cores are summarized in Tables 20 and 21 . Units and definitions will be found in Arts. 2, 3, 4

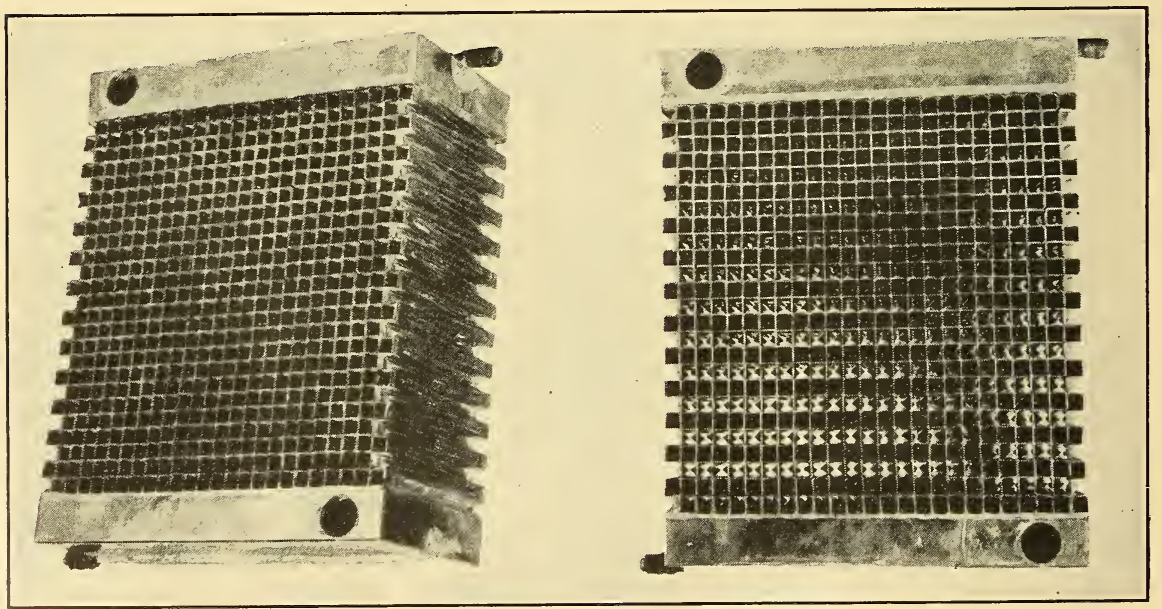

FIG. I2I.-Core section G-4 for which properties are shown in curves of Fig. I2O. The staggered arrangement of the tubes is shown in the perspective view at the right 


\section{APPENDIXES}

\section{APPENDIX A}

\section{IO. GENERAL NotATION}

$a=$ free area of radiator core.

$b=$ any constant; constant in equation $H=b M^{\mathrm{n}}$; width of fin.

$C_{\mathrm{p}}=$ specific heat of air or steam at constant pressure.

$c=$ conversion factor; constant in equation $R=c S^{2}$; correction for humidity, article I2.

$D=$ diameter of tube.

$E=$ effectiveness of indirect cooling surface; e. m. f. from thermocouples.

$e=$ base of Napierian logarithms.

$F=$ figure of merit.

$f=$ sign of a function.

$g=$ acceleration of gravity.

$H=$ heat dissipated, $\frac{\mathrm{h} . \mathrm{p} .}{\mathrm{sq} . \mathrm{ft} . \times 100^{\circ} \mathrm{F} \text {. }}$ or $\frac{\mathrm{kw}}{\mathrm{m}^{2} \times 50^{\circ} \mathrm{C}}$

$H$, with subscripts=heat dissipation in other units or particular values of $H$.

$k=$ any constant; constant in equation $M=k S$; thermal conductivity.

$l=$ length.

$M=$ air flow, $\frac{\mathrm{lb} \text {. }}{\mathrm{sec} \times \mathrm{sq} . \mathrm{ft} \text {. }}$ or $\frac{\mathrm{kg}}{\mathrm{sec} \times \mathrm{m}^{2}}$

$m=$ air flow constant.

n=exponent of equation $H=b M^{\mathrm{n}}$; number of plates per unit width in flat plate radiators; general symbol for exponent in a few other special applications.

$P=$ power absorbed; an integral used in calculation of air flow constant.

$p=$ pressure, with subscripts; perimeter of air tubes.

$q=$ surface coefficient of heat dissipation, heat per unit time per unit surface per unit temperature difference.

$R=$ head resistance; head resistance chargeable to the radiator.

$r=$ hydraulic radius; lift-drift ratio; relative humidity.

$S=$ flying speed.

$T=$ temperature difference between air and water; absolute temperature.

$t=$ time.

$u=$ see article 68 .

$v=$ velocity.

$w=$ weight of core and contained water; weight of steam condensed.

$x=$ depth of radiator; distance along air tubes; gage readings; differential pressure of pitot tube gage.

$y=$ thickness of fin.

$\alpha=$ see article 63 .

$\rho=$ air density.

\section{APPENDIX B.-TURBULENCE IN AIR TUBES}

III. EXPERIMENTAL Methods Úsed.-No attempt is here made to take up the questions of turbulence from a theoretical standpoint, but a brief record of experimental evidence bearing on the problems is presented, ${ }^{31}$ together with certain con-

31 A detailed record of these experiments has been published as Technical Report No. I06, National Advisory Committee for Aeronautics. 
clusions that seem to be warranted by the evidence, namely, that turbulent flow is found in the tubes of ordinary radiators at usual speeds, and that there are differences in nature or degree of turbulence in different radiators. Three general methods may be used for detecting turbulence-a visual method using some kind of smoke, measurements of pressure gradients, and measurements of heat transfer or of temperature.

The visual method has great advantages, but is inconvenient for work inside of the radiator because of the difficulty of arranging the apparatus so that it shall not disturb the flow of air and at the same time so that the air currents in the radiator tubes may be distinguished from currents before and behind it. The fact that the pressure gradient along an air stream is approximately proportional to the first power of the speed for stream line or viscous flow and to the square of the speed for turbulent flow may be used to determine the nature of the stream. The transfer of heat from a surface swept by a stream of air depends upon the turbulence of the stream as well as upon its velocity, and while with the present limited knowledge of coefficients of heat transfer, a single measurement might be of little value for detecting the presence or absence of turbulence considerable information may be gained from comparative measurements. Temperature measurements at different points in a stream of air that is being heated or cooled may, if reliable, furnish some indication of the condition of the air by showing how heat is transmitted through different portions of the stream.

The experimental work on which the conclusions are based was done in a $20 \mathrm{~cm}$ (8-inch) square wind tunnel, and the evidence here presented is not sufficient for a confident answer to the question whether the same kind of flow is found in a stationary radiator with air blowing through it as in a radiator moving through still air. It seems to be shown conclusively, however, that, at least when the radiator is in the wind tunnel, there are characteristic conditions of turbulence in different types of core, and it seems reasonable to suppose that such characteristic conditions would also be found in radiators moving through still air. The experimental work undertaken for the investigation of turbulence in radiator tubes includes the following parts:

I. Pressure gradients in radiator tubes.

II. Cooling coefficients of radiators.

III. Cooling of wires in an air stream.

IV. Temperature gradients.

II2. PRESSURE GRADIENTS.-An indication of turbulent flow is given in Table 2 (art. I7) by the agreement between the power of the air flow to which the pressure gradient is proportional and that computed by the Lees formula for turbulent flow in long tubes.

iI3. SURFACE HEat Dissipation CoefFicients of Radiators.-The coefficient $q$ of Table 2I, which represents heat dissipated per unit time per unit surface per unit temperature difference at an average speed of roo feet per second ( $30 \mathrm{~m}$ per sec) through the tubes, varies widely between different types of radiators. Reference to the table will show that the coefficient ranges (using the English units) from 0.7 to I.I for radiators whose air passages have straight, smooth walls, from 0.7 to I.I for pseudocellular types, from 1.0 to 2.2 for perforated plate types that whistle in an air stream, and for a type with spiral vanes is I.3. In general, the coefficient decreases as the size of the cell increases.

The wide range of coefficients even for straight tubes, the high coefficient for the section with spiral vanes, and the very high values found with some of the perforated plate types appear to be very strong evidence of varying conditions of turbulence in the different classes. The presence of a peculiar turbulence in the perforated plate types is also indicated by their whistle.

Coefficients for the radiators with true circular air tubes (type $\mathrm{C}-2$ ) if computed by the equation of Nusselt ${ }^{32}$ would be 0.003 I, the observed value for $\mathrm{C}-2$ at the same air speed being 0.0024 . Nusselt's equation represents conditions with turbulent flow in

${ }^{32}$ Zeitschrift des Vereines Deutscher Ingenieure, 53 II, I909, p. x81x; 54 II, x910, p. xx54. 
the central portions of long tubes, whence its application to radiator tubes is somewhat open to question.

II4. CoOling OF WIRES IN AN AIR STREAM.-The temperatures maintained by electrically heated wires connected in series so that each should carry the same current were used for a comparison of the cooling effect of the small streams of air flowing through a cold radiator with that of the stream in the wind tunnel in front of it.

Two pieces of $0.1 \mathrm{~mm}$ ( 0.004 inch) platinum wire, each about $6 \mathrm{~cm}(2.4$ inches) long and separated by $48 \mathrm{~cm}$ (I9 inches) of No. 36 copper wire, were strung by copper leads straight along the channel, so that one wire was in the stream some distance in front of the radiator, while the other was in one of the tubes, well back toward the rear face. The platinum wires, connected in series with a current-measuring shunt, as shown in Fig. r22, were heated by a small electric current and the potential drop across each platinum wire and across the shunt measured with a potentiometer. In order that the wires might disturb the air flow as little as possible, the potential and current leads were laid side by side (not twisted) and shellacked together for some distance from the platinum wires. The current leads supporting the wires were

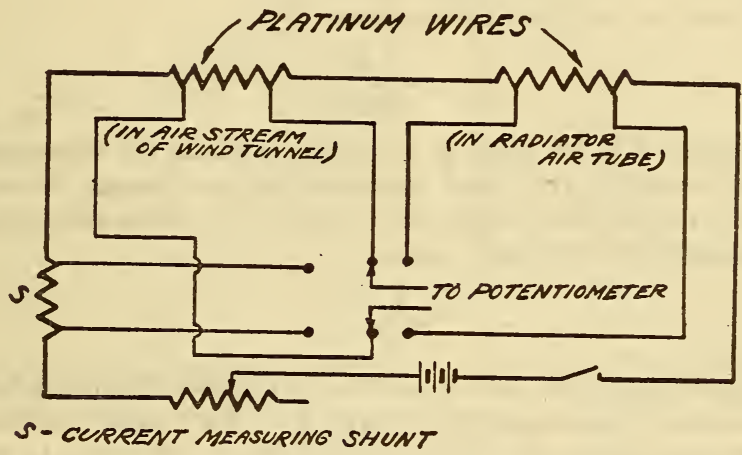

FIG. I22.-Diagram of electrical connections for the comparison of the cooling effect of the air stream in a radiator air tube and the cooling effect of the open stream in the wind tunnel some distance in front of the radiator core

Platinum wires in each of the two positions were heated by the same current and their equilibrium temperatures were measured by their resistances, obtained by a potentiometer method

passed over rods set across the channel about $55 \mathrm{~cm}(22$ inches) in front of and behind the radiator and out through small holes in the floor of the wind tunnel. The resistances when unheated were measured with small currents (about 0.006 ampere) and in an air stream of velocity about $\mathrm{I}_{3}$ meters per second ( 30 miles per hour). It seemed hardly worth while to attempt to use the wires as resistance thermometers to the extent of measuring the temperatures to which they rose, but since for small temperature changes the resistance of the platinum wire is roughly proportional to the absolute temperature, the fractional increase in resistance of each wire was used as a rough measure of its rise in temperature on being heated.

Heat dissipation from fine wires to moving fluids has been found to be proportional to the temparature difference and the square root of the velocity for a silver wire in a tube of flowing water; ${ }^{33}$ and for a platinum wire moved through air on a whirling arm, ${ }^{34}$ and if the same relation is assumed for the present case, a factor depending upon the turbulence may be found as follows: The relation may be expressed by the equation

$$
H=c \theta \sqrt{v}
$$

${ }^{33}$ Rogovsky, Comptes Rendus, r36, June 8, r903, p. r39r.

${ }^{34} \mathrm{King}$, Philosophical Transactions of the Royal Society of London, A214, 1914, p. 373. 
where $H=$ heat dissipated from the wire in units of power per unit length of wire.

$\theta=$ temperature difference between wire and air.

$v=$ linear velocity of air past wire.

$c=\mathrm{a}$ coefficient which includes a factor representing degree or nature of turbulence.

The air velocity is greater past the rear wire than past the front wire, because the radiator restricts the cross section of the stream and

$$
v_{2}=\frac{v_{1}}{a}
$$

where $a=$ the fractional part of the frontal area of the radiator that is open for the passage of air, called its "free area."

and the subscripts $\mathrm{I}$ and 2 represent the front and rear wires, respectively. Turbulence at the front wire-i. e., in the open channel of the tunnel-is represented by the coefficient

$$
c_{1}=\frac{H}{\theta_{1} \sqrt{v_{1}}}
$$

and at the rear wire-i. e., in the radiator tube-by

$$
c_{2}=\frac{H}{\theta_{2} \sqrt{v_{2}}} .
$$

The heat dissipation $H$ is the same in both cases, because the wires carry the same current and are practically the same resistance per unit length. A comparison of turbulence in the radiator with that in front of it is $\mathrm{o}^{\text {r+ }}{ }^{-}-\mathrm{d}$ by substituting equation (2) in (4) and dividing by (3), which gives

$$
\frac{c_{2}}{c_{1}}=\frac{\theta_{1}}{\theta_{2}} \sqrt{a}
$$

As indicated above, the per cent of increase in resistance on being heated is used as a rough measure of the temperature difference $\theta$. The values, including the ratio of the coefficients, are shown in Table 22 and indicate differences in turbulence in different types of radiator with the greatest turbulence in the perforated plate type, which whistles in an air stream.

\begin{tabular}{|c|c|c|c|c|c|c|c|}
\hline \multirow{2}{*}{ Type of radiator } & \multirow{2}{*}{$\underset{\text { mate) }}{\text { Air speed }}$} & \multirow{2}{*}{ Current } & \multicolumn{2}{|c|}{$\begin{array}{l}\text { Per cent increase } \\
\text { in resistance }\end{array}$} & \multirow{2}{*}{$\begin{array}{c}\text { Free area } \\
a\end{array}$} & \multirow{2}{*}{$\begin{array}{c}\text { Ratio of } \\
\text { coeffi- } \\
\text { cients } \\
\frac{\theta_{1}}{\theta_{2}} \sqrt{a}\end{array}$} & \multirow{2}{*}{$\begin{array}{c}\text { Mean } \\
\text { ratio }\end{array}$} \\
\hline & & & $\underset{\theta_{1}}{\text { Front }}$ & $\underset{\theta_{2}}{\text { Rear }}$ & & & \\
\hline \multirow{4}{*}{ Flat plate...$\ldots \ldots \ldots \ldots \ldots$} & $\mathrm{m} / \mathrm{sec}$ & amp. & Per cent & Per cent & Ratio & & \\
\hline & 13 & 0.285 & 2.3 & 2. 1 & 0.88 & 1.05 & ........... \\
\hline & 13 & .457 & 8.1 & 7.8 & (........... & .98 & (........... \\
\hline & 18 & .456 & 7.4 & 7.0 & (.......... & 1.00 & 1.01 \\
\hline \multirow[t]{5}{*}{ Circular cell .................... } & 13 & .485 & 9.6 & 9.5 & .65 & .81 & (........... \\
\hline & 17 & .472 & 9.3 & 8.5 & ........... & .88 & ........... \\
\hline & 13 & .304 & 3.7 & 3. 3 & ........... & .90 & .......... \\
\hline & 17 & .303 & 3.6 & 3.3 & ........... & .88 & .......... \\
\hline & 17 & .497 & 9.9 & 9.0 & ........... & .83 & .87 \\
\hline \multirow[t]{4}{*}{ Perforated plate................. } & 18 & .208 & .8 & .5 & ๑ 88 & 1.7 & .......... \\
\hline & 18 & .407 & 5.9 & 4. 4 & .......... & 1.26 & ........... \\
\hline & 13 & .404 & 5.5 & 4.0 & ........... & 1.30 & ........... \\
\hline & 13 & .448 & 7.6 & 5.6 & ............ & 1.29 & 1. 30 \\
\hline
\end{tabular}

TABLE 22.-Comparison of Turbulence by Relative Cooling of Two Wires in the Air Streams

(1) In open channel of wind tunnel, (2) in the air tube of a radiator core 
II5. Temperature Gradients in Air Tubes.-In order to obtain some indication of the distribution of temperature within the air passages of radiators, the following procedure was followed: A section of radiator was mounted in the $20-\mathrm{cm}$ (8-inch) wind tunnel and hot water was pumped through it as in calorimetric tests. A copper-constantan thermocouple was strung through one of the air tubes of the radiator and supported by its own copper leads in the manner described above for the platinum wires. The constantan wire was about $30 \mathrm{~cm}$ (12 inches) long, and the cold (upstreamjunction was outside of the radiator in the stream of incoming air, when the hot (down) stream) junction was in any measured position in the radiator tube or even somewhat behind the rear face. Screw threads, with a pitch of $0.16 \mathrm{~cm}(\mathrm{I} / \mathrm{r} 6 \mathrm{inch})$ on the rods
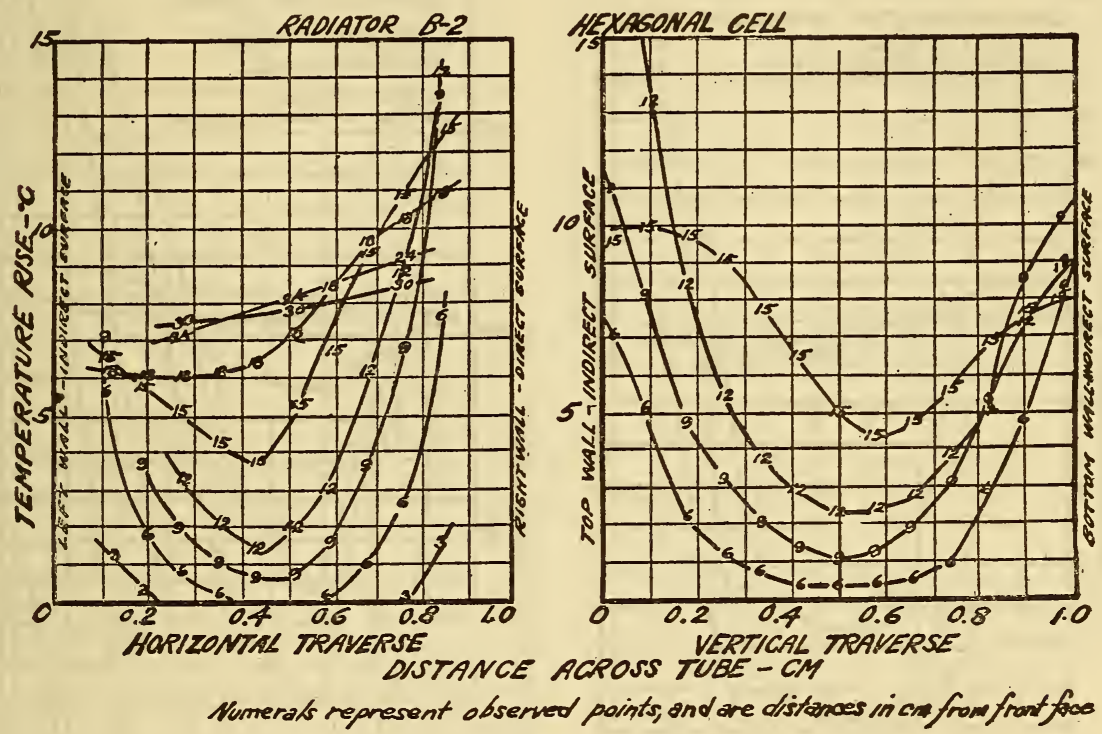

FIG. I23.-Temperature gradient across air tube of a hexagonal cell radiator in several planes at intervals of $3 \mathrm{~cm}$

The numerals represent observed points and indicate the distances of the respective planes back from the front face of the radiator. The core is $10.2 \mathrm{~cm}$ deep, and mapping of the gradients was continued far beyond the rearface of the radiator, the last plane shown in the figure being $30 \mathrm{~cm}$ back of the front face. The vertical boundaries of the plot mark the position of the walls of the air tube. Average water temperature was $55^{\circ} \mathrm{C}$ above entrance air temperature, but the tube walls formed by indirect cooling surface were at temperatures somewhat lower. Lack of symmetry in horizontal traverse is due to the fact that the right wall is direct and the left indirect, cooling surface. Lack of symmetry in vertical traverse is probably due to a slight error in alignment of radiator and thermocouple wire

supporting the wires and on the supports for the rods, furnished rough micrometers for setting the position of the thermocouple and for moving it horizontally and vertically.

The mean temperature difference between the water in the radiator and the air passing through it and the speed of the air stream were maintained approximately constant, and corrections for variations in the temperature difference were made on the assumption that the temperature rise indicated by the thermocouple was proportional to this difference. No correction was made for slight fluctuations in the speed of the air stream, for trial showed that the effect on the thermocouple readings was small, even when the speed was varied over a wide range.

In order that the air might be disturbed as little as possible, fine wires were used and were bared for some distance each side of the constantan section. At first wire of 
diameter o.10 cm (B. \& S. gage 38 ) was tried, but so much trouble was experienced with breakage that most of the work was done with wire of diameter $0 . \mathrm{I}_{3} \mathrm{~cm}(\mathrm{~B}$. \& $\mathrm{S}$. gage 36 ).

Figures $123-5$ show temperature gradients across the center of the tubes in four radiators, in planes at different distances from the front face, the sides of the plots representing the walls of the tubes. Figs. 126-7 show isothermal lines in a diameter plane of an air tube plotted from the data shown on the other curves, and the upper

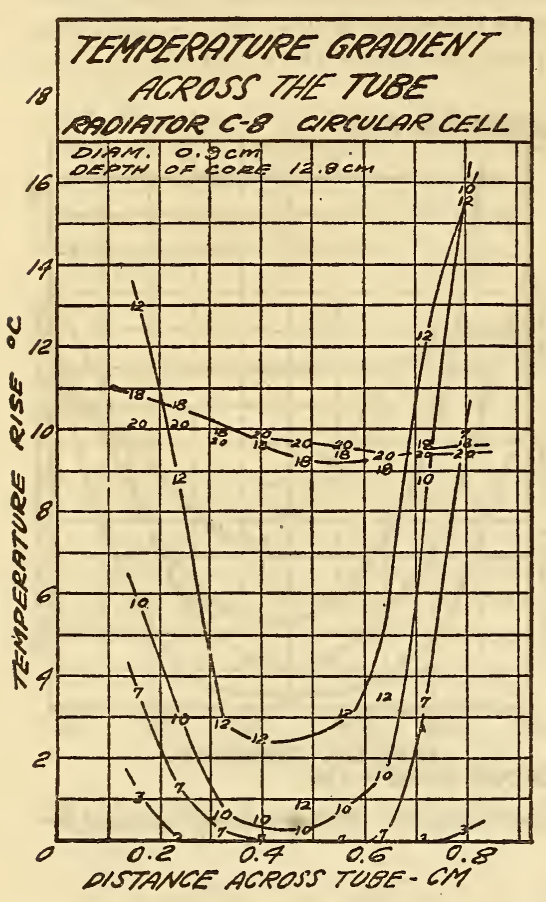

FIG. I24.-Temperature gradients across air tubes of circular cell radiators, in several planes at different distances back from the front face of the core

The numerals represent observed points and indicate the distance of the respective plane back of the front face. It will be noted that gradients are mapped in the open wind stream in planes considerably back cf the back faces of the cores. The vertical boundaries cf the plots mark positions of the walls of the tubes. Average water temperature $55^{\circ} \mathrm{C}$ above temperature of air entering the core

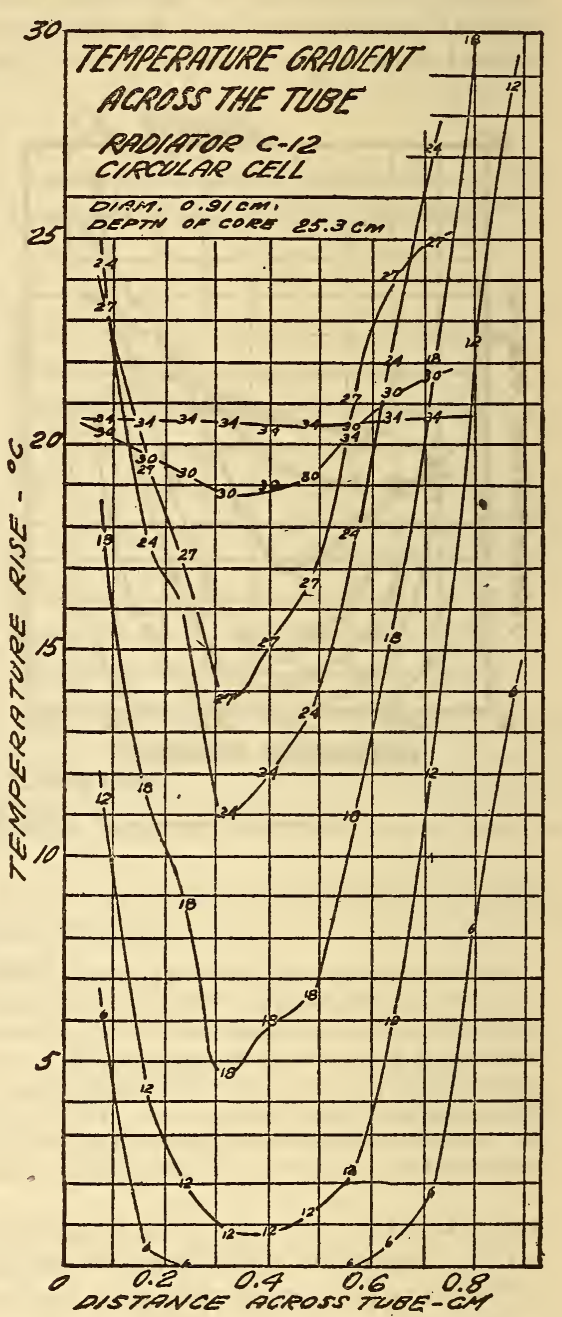

and lower sides of the plots indicate the walls of the tubes. It must be emphasizea that the quantities shown on the curves are very rough values and can be used quantitatively only with very great caution, if at all, because the steepness of the temperature gradients across the tube and the uncertainty in the position of a thermoelectric junction suspended by a meter of fine wire in an air stream make individual readings quite unreliable. Indeed, the uncertainty of position made it impossible to duplicate readings with any accuracy after the wires had been moved forward or backward, and readings were accordingly taken across the tube from one side to the other before 
moving the thermocouple to a new position along the stream. But, although individual readings are not very reliable, the qualitative indications of the plots are probably correct. ${ }^{35}$

It has been suggested that at sections of the tubes near the forward end both viscous and turbulent flow might exist, each occupying a certain part of the cross section. If such were the case, a sudden break would be expected in the temperature gradient across the tube, at the boundary between the two kinds of flow, but no indication of

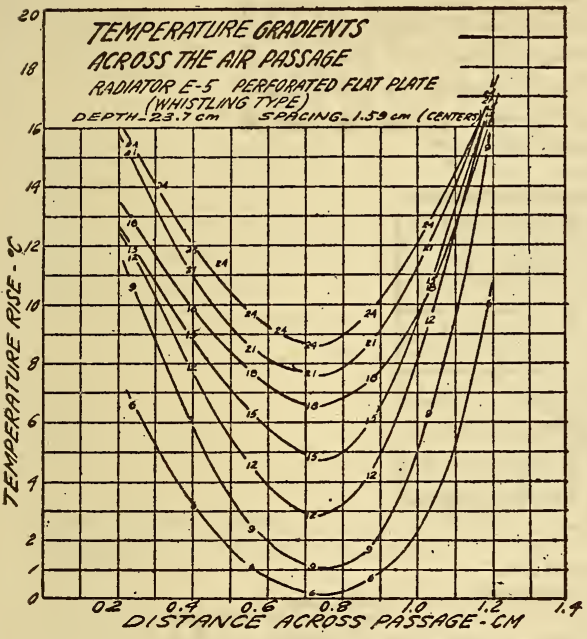

FIG. 125.-Temperature gradients across air passages of a flat plate core construction, with perforations in the plate (whistling type)

The numerals represent observed points and indicate the distance back from the front face to the plane in which the gradient is mapped. The vertical boundaries of the plot indicate the position of the side walls of the air passage. The average water temperature was $55^{\circ} \mathrm{C}$ above the temperature of the air at the front face of the radiator

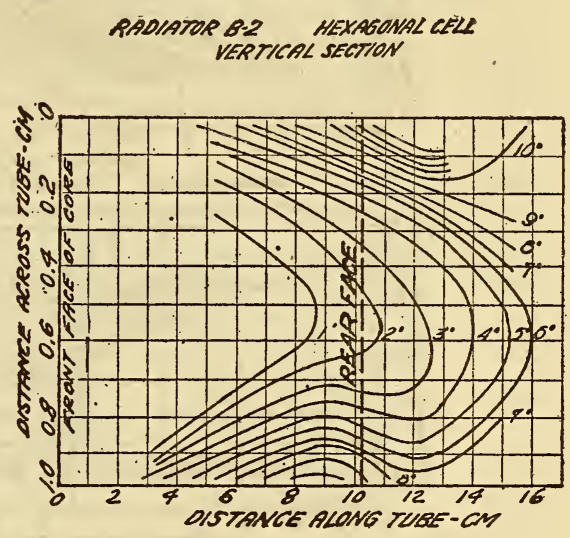

FIG. I26.-Isothermal lines in the vertical diameter plane of an air tube of a hexagonal cell radiator core

Plotted from data shown in Fig. I23. Lack of symmetry is chargeable to the inaccuracies of reading described in Article 114. Average water temperature $55^{\circ} \mathrm{C}$ above temperature of air entering the core

such a break is found in the data; and, although the inability to get reliable readings very close to the walls (because of contact between the swinging thermocouple wire and the wall) might have concealed this condition in some parts of the tubes, it seems reasonable to suppose that it would have been detected at least in the longest tube.

The curves are of the form characteristic of turbulent flow and are similar to curves of temperature gradients ${ }^{36}$ and isothermal lines ${ }^{37}$ found by other observers when working with long tubes at velocities well above the critical value.

35 The possible effect of errors due to lead conduction in the thermocouple was investigated and found to be entirely negligible in comparison with the known errors due to uncertainty of position.

${ }^{36}$ T. E. Stanton and Dorothy Marshall, British Advisory Committee for Aeronautics, Reports and Memoranda, No. 243, June, I9I6.

${ }^{37}$ Groeber, Zeitschrift des Vereines Deutscher Ingenieure, 56, March, I912, p. 421. 


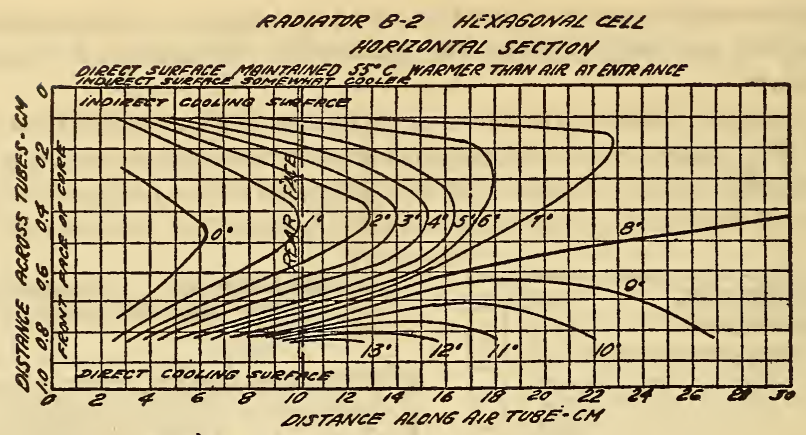

RAOIOTTR CO CIRCULAR CELL

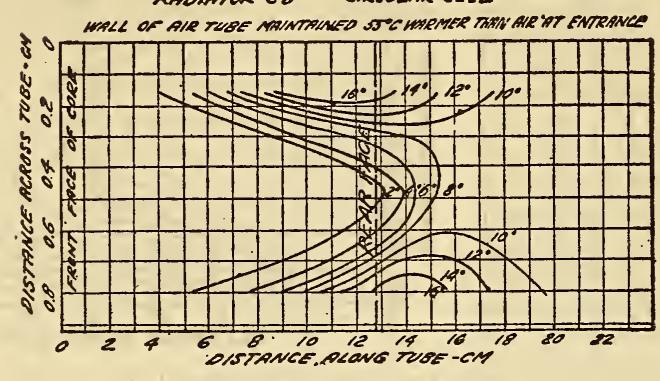

RAOIATOP C.IL CIRCULAR CELL

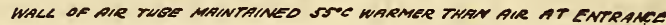
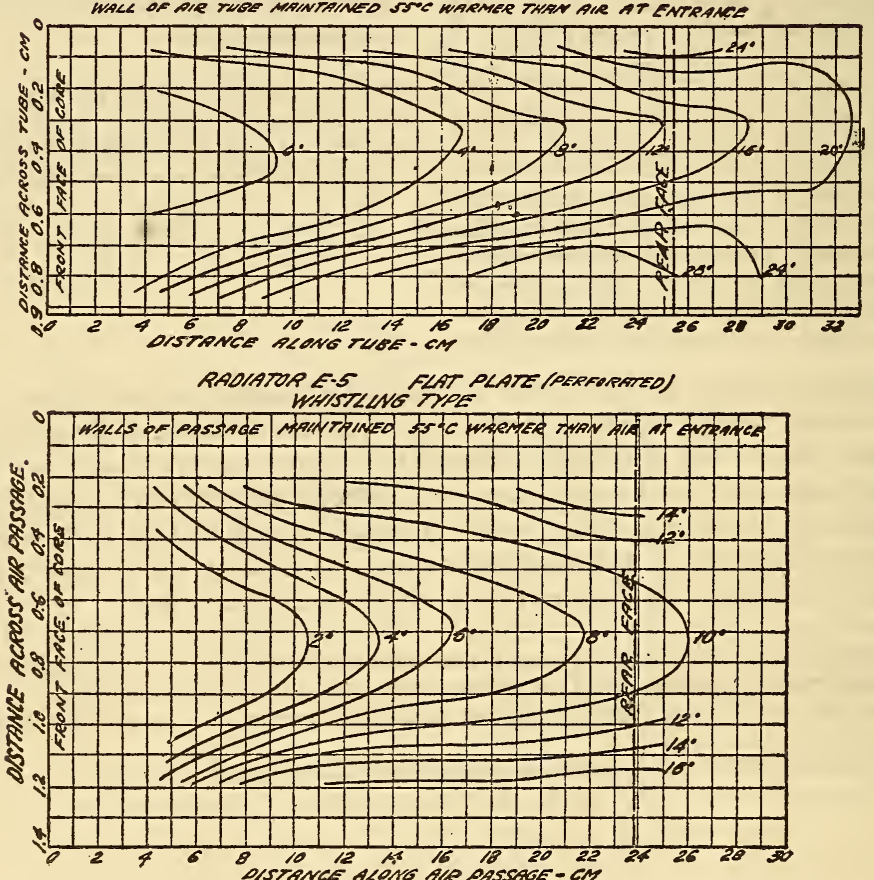

FIG. I27.-Isothermal lines in a horizontal diameter plane of an air tube fordifferent types of core. Plotted from data shown in Figs. I23-125 


\section{APPENDIX C.-REDUCTION FOR AIR DENSITY IN COMPUTATION OF AIR- FLOW CONSTANT}

II6. Extending the notation of article I7, let

$M=$ air flow, units of mass per unit time per unit frontal area of radiator,

$M_{0}=$ "unobstructed" air flow in open wind tunnel,

$\rho=$ air density,

$Q=$ pressure difference between two points in the air tube of the radiator core,

$b^{\prime}, n=$ constants for each radiator,

$c=$ calibration factor of the pitot tube, including the usual coefficient of the tube and conversion factors incident to units chosen,

$h=$ reading on pressure gage,

and let the subscripts I and 2 denote the closed or steam tunnel and the open 54 -inch tunnel, respectively. (In the tunnel (I) the radiator core fills the wind channel completely, so that the entire wind stream is forced to pass through the core; in (2) the relative transmission through the core and deflection around it are conditioned by the core construction.)

By equation (6), article I7,

and

$$
Q_{1}=b \frac{M^{\mathrm{n}}}{\rho_{1}}
$$

$$
Q_{2}=b^{\prime} \frac{M^{\mathrm{n}}}{\rho_{2}} .
$$

In the steam tunnel $Q_{1}$ and $M_{1}$ are measured, and equation ( $\mathrm{I}$ ) may be solved for $\mathrm{b}^{\prime}$, giving

Solving equation (2) for $\mathrm{M}_{2}$,

$$
b^{\prime}=\frac{Q_{1} \rho_{1}}{M_{1}^{\mathrm{n}}}
$$

$$
M_{2}=\sqrt[n]{\frac{Q_{2} \rho_{2}}{b^{\prime}}}
$$

and substituting (3) in (4)

$$
M_{2}=\sqrt[n]{\frac{Q_{2} \rho_{2}}{Q_{1} \rho_{1}}} M_{1}
$$

Now, the air flow constant is by definition

$$
m=\frac{M_{2}}{M_{0}}
$$

and the equations of the pitot tubes may be put in the forms

$$
\begin{aligned}
& M_{1}=c_{1} \sqrt{\rho_{1} h_{1}} \\
& M_{0}=c_{2} \sqrt{\rho_{2} h_{2}}
\end{aligned}
$$

Substituting (7) in (5) and (8) and (5) in (6),

$$
m=\sqrt[n]{\frac{Q_{2} \rho_{2}}{Q_{1} \rho_{1}}} \frac{c_{1}}{c_{2}} \sqrt{\frac{\rho_{1} h_{1}}{\rho_{2} h_{2}}}
$$

In the computation as outlined in article $I 7$ it is assumed that $\rho_{1}=\rho_{2}$ and the error introduced in this equation by that assumption is evidently indicated by the quantity

$$
\sqrt[n]{\frac{\rho_{2}}{\rho_{1}}} \sqrt{\frac{\rho_{1}}{\rho_{2}}}=\left(\frac{\rho_{2}}{\rho_{1}}\right)^{\frac{2-n}{2 n}}
$$

The value of $n$ hardly ever falls below I. 7 , and a change in density from 0.0750 to 0.0700 pounds per cubic foot represents a wide variation in conditions. For this extreme case the correction factor becomes

$$
\left(\frac{0.0750}{0.0700}\right)^{0.09}=1.006
$$




\section{APPENDIX D.-TEMPERATURE DROP IN WATER-TUBE WALLS}

II7. To support the statement of article $5^{8}$ that the temperature difference between inside and outside of water-tube walls is small in comparison with the temperature difference between air and water, an estimate of the magnitude of the drop in the metal may be made by estimating the heat transfer, assuming an amount of cooling surface and computing the temperature head necessary to force heat through various thicknesses of metal at the estimated rate.

As an extreme case, assume a flying speed of 200 miles per hour, or 89.4 meters per second, air density of $\mathrm{I} .2 \mathrm{~kg}$ per $\mathrm{m}^{3}$ ( 0.0750 pounds per cubic foot), an air flow constant of 0.84 , specific heat of 0.24 , cooling surface 20 times the frontal area, and a rise in air temperature of $27.7^{\circ} \mathrm{C}$ or $50^{\circ} \mathrm{F}$ in passing through the radiator.

Then, the heat taken up by the air per square meter frontal area of core is $89.4 X$ $0.84 \times 1.2 \times 0.24 \times 27.7=600$ kilogram calories per second, or per square foot frontal area is $293 \times 0.84 \times 0.0750 \times 0.24 \times 50=222$ Btu per second.

Taking the thermal conductivities ${ }^{38}$ of copper, brass, aluminum, and iron, respectively, as $0.9 \mathrm{I}, 0.26,0.49$, and $0 . \mathrm{I}_{4}$, in $\frac{\mathrm{cal}}{\mathrm{cm} \times \sec \times{ }^{\circ} \mathrm{C}}$ and computing the temperature difference required to conduct heat at this rate through 20 square meters of metal of various thicknesses, Table 23 is computed.

TABLE 23.-Temperature Drop Through Metal of a Radiator Core under Extreme Conditions (Conditions Stated in Art. 117)

\begin{tabular}{|c|c|c|c|c|c|c|c|c|c|}
\hline \multirow{2}{*}{$\begin{array}{l}\text { Thickness } \\
\text { of metal }\end{array}$} & \multicolumn{4}{|c|}{ Temperature drop through metal } & \multirow{2}{*}{$\begin{array}{l}\text { Thickness } \\
\text { of metal }\end{array}$} & \multicolumn{4}{|c|}{ Temperature drop through metal } \\
\hline & Copper & Brass & Alumi- & Iron & & Copper & Brass & $\begin{array}{c}\text { Alumi- } \\
\text { num }\end{array}$ & Iron \\
\hline $\mathrm{cm}$ & ${ }^{\circ} \mathrm{C}$ & ${ }^{\circ} \mathrm{C}$ & ${ }^{\circ} \mathrm{C}$ & ${ }^{\circ} \mathrm{C}$ & Inches & ${ }^{\circ} \mathrm{F}$ & ${ }^{\circ} \mathrm{F}$ & ${ }^{\circ} \mathrm{F}$ & ${ }^{\circ} \mathrm{F}$ \\
\hline 0.01 & 0.033 & 0.12 & 0.06 & 0.21 & 0.005 & 0.075 & 0.26 & 0.14 & 0.49 \\
\hline .02 & .07 & .23 & .12 & .43 & .010 & .15 & .53 & .28 & .98 \\
\hline .03 & .10 & .35 & .18 & .64 & .015 & .23 & .79 & .43 & 1.5 \\
\hline .04 & .13 & .46 & .25 & .86 & .020 & .30 & 1.1 & .56 & 2.0 \\
\hline .05 & .16 & .58 & .31 & 1.07 & 025 & .37 & 1.3 & .70 & 2.5 \\
\hline .06 & .20 & .69 & .37 & 1.3 & .030 & .45 & 1.6 & .84 & 2.9 \\
\hline .07 & .23 & .81 & .43 & 1.5 & & & & & \\
\hline
\end{tabular}

\section{APPENDIX E.-CHOICE OF TEMPERATURE DIFFERENCE TO BE USED IN EXPRESSING RESULTS OF HEAT DISSIPATION MEASUREMENTS}

II8. The comparatively small change in temperature of the water passing through a radiator makes it relatively easy to compute or estimate the effective average temperature under any probable conditions of use. This is not true for the air, and it would be distinctly inconvenient to have data for a given type of core expressed in terms involving the average temperature of the air, because in any use of such data it is only the entrance air temperature which is specified. The average can not, in general, be computed quickly or estimated, because too many factors enter into fixing the exit air temperature. For the intercomparison of radiator cores it is found possible to express the results of measurements conveniently in terms of entrance air temperature and average water temperature without in any way affecting the accuracy of expression of relative magnitudes. The algebraic proof of this follows.

Assume a particular radiator under conditions of constant air flow and water flow but with varying temperatures and consequently varying heat dissipation. It may

${ }^{38}$ Kaye and Laby, Physical and Chemical Constants, p. 5 I. 
also be assumed for work with not too great temperature differences that the heat dissipation is proportional to the difference between the mean water temperature and the arithmetical mean of the temperatures of the air at entrance and exit. This assumption is, of course, not strictly accurate, but is sufficiently close for the kind of work required in radiator tests.

Let

$H=$ heat dissipated, in units of power.

$T_{\mathrm{w}}=$ mean temperature of water.

$T_{1}=$ temperature of air at entrance.

$T_{2}=$ temperature of air at exit.

$T=T_{\mathrm{w}}-T_{1}$.

$k=\mathrm{a}$ constant.

Also, let accented and unaccented symbols denote, respectively, two sets of values under conditions similar, except that the temperatures may be different in the two cases. The assumption stated above may be expressed in the equation

$$
\bar{H}=\frac{T^{\prime}{ }_{\mathrm{w}}-\frac{T_{1}^{\prime}+T_{2}^{\prime}}{2}}{T_{\mathrm{w}}-\frac{T_{1}+T_{2}}{2}} .
$$

The heat taken up by the air is evidently

from which

$$
H=k\left(T_{2}-T_{1}\right)
$$

$$
T_{2}=T_{1}+\frac{H}{k}
$$

and from equation (3) equation ( $\mathrm{I}$ ) may be written

$$
\frac{H^{\prime}}{H}=\frac{T_{\mathrm{w}}^{\prime}-T_{1}^{\prime}-\frac{H^{\prime}}{2 k}}{T_{\mathrm{w}}-T_{1}-\frac{H}{2 k}}
$$

from which

$$
\frac{H^{\prime}}{H}=\frac{T^{\prime}-\frac{H^{\prime}}{2 k}}{T-\frac{H}{2 k}}
$$

Now letting

$$
\begin{gathered}
\frac{H}{2 k}=A \\
\frac{H^{\prime}}{H}=\frac{T^{\prime}-\frac{H^{\prime}}{H} A}{T-A}
\end{gathered}
$$

which is true only when

$$
\frac{H^{\prime}}{H}=\frac{T^{\prime}}{T}
$$

For ordinary purposes and under ordinary conditions of use the difference between the true mean temperature of the water and the arithmetical mean of the temperatures of the water at entrance and exit is so small that the arithmetical mean may be used safely. 


\section{APPENDIX F.-DERIVATION OF EQUATION FOR EFFECTIVENESS OF INDIRECT COOLING SURFACE}

Irg. The effectiveness of indirect cooling surface is defined as the ratio of its rate of dissipation of heat to that of an equal area of direct surface under the same conditions of air flow, but at a temperature equal to that of the parts of the indirect surface that are adjacent to its source of heat.

The form of "fin" to be considered is one found in many cellular types of radiator, extending from front face to rear face of the radiator and from one water tube to another. The fins of "fin-and-tube" types present quite a different mathematical problem, and since they are of little importance in aeronautic work will not be considered here.

Two cases of longitudinal fins will be distinguished: (I) Fin in good thermal contact with both water-tube walls, i. e., along both long edges of fin; and (2) fin in good thermal contact with only one water-tube, i. e., along only one edge of fin. Fins of the first class are found in the radiator $\mathrm{C}_{-} 4$ and of the second class in many of the square-cell types.

In each case it will be assumed (x) that the depth of the water tube is so nearly equal to the depth of the radiator as to justify neglect of any inequality, (2) that the fin is long in comparison with its width, and (3) that the temperature of the fin does not vary appreciably with depth of the radiator, although decreasing across the width of the fin.

An analysis similar to that of Appendix E would show that if heat dissipation of any element of cooling surface is proportional to the difference in temperature between such an element and the air passing it, the heat dissipation is also proportional to the difference in temperature between the surface and the entering air, provided that the temperature distribution be such that the arithmetical mean of entrance and exit air temperatures is a reliable substitute for the mean effective air temperature, properly averaged over the whole surface. The assumption is but a rough approximation to the truth, as indicated by Figs. I23-127, but it is good for the purposes of this article. The conclusions reached would not be altered greatly by the still more sweeping assumption of no appreciable change at all in the air temperature as the air traverses the tubes. For convenience all temperature differences will be referred to entering air, and in the mathematical treatment the temperature scale will be assumed so chosen as to make the temperature of the entering air equal to zero.

i20. Case I. Thermal Contact on Both Sides-In accordance with assumptions (I) and (2) the effects of the ends of the fins will be neglected and the problem reduces to the case of a long, thin metal strip with constant temperature at each long edge where the strip is in metallic contact with the water tube. The flow of heat is practically unidimensional from the edges toward the center line of the fin, and if the fin is uniform in thickness and conductivity, and the temperature the same at the two edges, it is clear that no heat will flow across the center line of the fin and the temperature gradient will vanish along that line. Under these conditions the heat dissipated by the fin may be found as follows:

Using any consistent units, let

$x=$ distance measured across the fin, away from one water-tube wall.

$b=$ width of fin (distance between water tubes).

$y=$ thickness of fin.

$l=$ length of fin.

$s=$ cross sectional area of fin over a section normal to the direction of flow of heat.

$p=$ perimeter of the section $s$.

$k=$ thermal conductivity of the fin.

$q=$ heat dissipated from surface of fin in units of heat per unit time, per unit surface, per unit temperature difference between surface and entering air.

$\theta=$ temperature of metal of fin (temperature of entering air assumed zero). 
$\theta_{0}=$ temperature of water-tube wall.

$Q=$ total heat dissipation of fin in units of heat per unit time.

$E=$ effectiveness of fin.

Considering a section through the fin at a distance $x$ from one water-tube wall, the heat conducted per unit time through the fin across this section is equal to

$$
k s \frac{d 6}{d x}
$$

and the heat conducted past a near-by section at the distance $(x+\Delta x)$ from the wall is

$$
k s\left(\frac{d \theta}{d x}+\frac{d^{2} \theta}{d x^{2}} \Delta x\right) .
$$

The heat dissipated from the surface between these sections is the difference between (I) and (2), or

and is also equal to

$$
k s\left(\frac{d^{2} \theta}{d x^{2}}\right) \triangle x
$$

Equating (3) and (4) and letting

gives

$$
\begin{aligned}
& q 9 p \Delta x . \\
& \alpha=\sqrt{\frac{q p}{k s}}
\end{aligned}
$$

$$
\frac{d^{2} \theta}{d x^{2}}=\alpha^{2} \theta
$$

A solution of this, equation may be put in the form

$$
\theta=A \cosh (\alpha x-B)
$$

where $A$ and $B$ are constants which may be evaluated from the boundary conditions

$$
\begin{aligned}
\theta & =\theta_{0} \text { when } x=O \\
\frac{d \theta}{d x} & =O \text { when } x=\frac{b}{2}
\end{aligned}
$$

These constants are found to be

$$
\begin{aligned}
& B=\alpha \frac{b}{2} \\
& A=\frac{\theta_{0}}{\cosh \alpha \frac{b}{2}}
\end{aligned}
$$

The heat dissipated per unit time by the whole fin is given by

$$
\begin{aligned}
Q & =2 \int_{0}^{\frac{b}{2}} q \theta p d x \\
Q & =2 q p A \int_{0}^{\frac{b}{2}} \cosh (\alpha x-B) d x \\
& =\frac{2 q p A}{\alpha}[\sinh (\alpha x-B)]_{0}^{\frac{b}{2}} \\
& =\frac{2 q p \theta_{0}}{\alpha} \frac{\sinh \alpha \frac{b}{2}}{\cosh \alpha \frac{b}{2}}=\frac{2 q p \theta_{0}}{\alpha} \tanh \alpha \frac{b}{2}
\end{aligned}
$$


Now, the average heat dissipation for the fin per unit time, per unit surface, per unit temperature difference between water-tube wall and entering air is

$$
\frac{Q}{b p \theta_{0}}
$$

and the effectiveness of the fin is by definition the ratio of this quantity to the rate, $q$, of dissipation per unit area of water-tube wall.

$$
\begin{aligned}
E & =\frac{Q}{b p \theta_{0} q}=\frac{2 q p \theta_{0}}{b p \theta_{0} q \alpha} \tanh \alpha \frac{b}{2} \\
& =\frac{\tanh \alpha \frac{b}{2}}{\alpha \frac{b}{2}}
\end{aligned}
$$

or letting

$$
\begin{aligned}
& u_{1}=\alpha_{2}^{\frac{b}{2}}=\frac{b}{2} \sqrt{\frac{p q}{k s}} \\
& E=\frac{\tanh u_{1}}{u_{1}}
\end{aligned}
$$

Since the perimeter $p$ is practically equal to twice the length of the fin, the equation for $\alpha$ may be put in slightly more convenient form by means of the substitution

$$
\frac{p}{s}=\frac{2 l}{y l}=\frac{2}{y}
$$

and the value of $u_{1}$ may be put in the form

$$
u_{1}=\frac{\alpha b}{2}=\frac{b}{2} \sqrt{\frac{2 q}{y k}}
$$

i2 i. Case II. Thermal Contact on ONe Side OnLy.-If the fin is in good thermal contact with the water-tube walls along only one edge, a very good approximation may be made by neglecting the heat dissipated from the free edge, since the thickness of the fin is seldom greater than 2 per cent of its width. In this case the derivation is changed in the following respects:

(I) The conditions (8) become

$$
\begin{aligned}
\theta & =\theta_{0} \text { when } x=O \\
\frac{d \theta}{d x} & =O \text { when } x=b,
\end{aligned}
$$

(2) $B$ becomes $(\alpha b)$,

$$
Q=\int_{0}^{b} q \theta p d x
$$

which integrates to

$$
Q=\frac{q p \theta_{0}}{\alpha} \tanh \alpha b,
$$

(4) the effectiveness becomes

$$
E=\frac{\tanh \alpha b}{\alpha b},
$$

which, letting

$$
u_{2}=\alpha b=b \sqrt{\frac{2 q}{y k}}
$$

becomes

$$
E=\frac{\tanh u_{2}}{u_{2}} \text {. }
$$




\section{APPENDIX G.-EXPERIMENTAL DATA}

122. In this appendix are collected the laboratory data upon which are based the conclusions forming the body of this paper. Data which have been incorporated explicitly into the material of the foregoing pages are not repeated in the following tables and graphs, but are cross referenced, as, for example, in Table 30 . To reproduce log sheets of the laboratory work would require a volume many times as thick as this; and, in general, it will be noted that the material given is condensed to a single line for each experimental run in the laboratory.

After this paper was prepared for publication it developed that the funds available for printing would not permit of the publication of this appendix, the tables for which are therefore given by title. The reader who is interested in reviewing the vast amount of experimental laboratory data upon which conclusions in this paper are based may do so by consulting the original manuscript at the Bureau of Standards. The elimination of these data from the present paper is regretted, but there seems to be no other alternative, since the appropriation allotted to the Bureau for printing falls considerably short of that required to publish its scientific and technical work. If funds should become available in the near future, and there appears to be a demand for it, the appendixes to the present paper may then be published separately as a supplement.

Table 24. Summary of Data Obtained in Heat Dissipation Measurements. Measurements made in closed or "steam" tunnel using steam as source of heat for 64 cores, and using hot water as the source of heat for I2 cores.

Table 25. Summary of Heat Dissipation Data Obtained in Reduced Pressure Tunnel.

Table 26. Air Flow Data-Venturi Meter.

Table 27. Air Flow Data for Radiator in Nose of Model Fuselage.

Table 28. Air Flow and Air Flow Distribution in Streamlined Radiator.

Table 29. Observed Data for Study of Surface Friction in a Single Long Tube.

Table 30. Head Resistance Data.

Table 3r. Head Resistance of Cores Yawed at Various Angles.

Much data given graphically will be found on pages $409-426$.

\section{APPENDIX H.-BIBLIOGRAPHY}

Since a complete bibliography of subjects allied even most intimately with the subject matter of this paper would be a compendium of dictionary size, it has been difficult to choose a suitable limitation to listing. The authors have concluded that the interests of the reader would be served best by indicating the few papers which have proven of greatest value in their relation to the work. Specific reference to a number of these will be found scattered through footnotes in the several sections of the paper.

Black, A., Aircraft radiators, Society of Automotive Engineers, March 7, I9r9.

BryanT, L. W., and IRving, H. P., Experiments on the resistance and cooling of radiators. Great Britain Advisory Committee for Aeronautics, Reports and Memoranda, No. $22 \mathrm{I}$.

CARRIER, W. H., Rational psychrometric formulæ. Transactions, American Societyof Mechanical Engineers, I9II, pp. 1005-1053.

Gibson, A. H., Radiators for water-cooled aero engines. Great Britain Advisory Committee for Aeronautics. Internal Combustion Engine Subcommittee Reports, No. I75.

Griffiths, E., Effect of enameling on heat transmission. Great Britain Advisory Committee for Aeronautics. Light Alloy Subcommittee Reports, No. 93.

GroEbER, H., Der Wärmeübergang von Heisser Luft an Rohrwandlungen. Zeitschrift des Vereines Deutscher Ingenieure, 56, I., I9I2, pp. 42I-426. 
KING, L. V., On the convection of heat from small cylinders in a stream of fluid: Determination of convection constants of small platinum wires with applications to hot wire anemometry. Philosophical Transactions Royal Society of London, 2 I4, r9r4, pp. 373-432.

KREMLIN, A., Reports on tests simulating effect of radiator placed within a wing section with investigation of sheltering fins.

LEA, F. C., Effect of enameling on the conductivity of aluminum cylinders. Great Britain Advisory Committee for Aeronautics. Light Alloy Subcommittee Reports, No. 82 .

LEES, C. H., Flow of viscous fluids through smooth circular pipes. Proceedings Royal Society of London, A-91, I914, pp. 46-53.

LIPTROT, Radiators and cooling systems for aircraft engines. Aeronautics, April 29, May 6, r920,

NUSSELT, W., Die Abhängigkeit der Wärmeübergangszah1 von der Rohrlänge. Zeitschrift des Vereines Deutscher Ingenieure, 54, II, I910, pp. II54-II 58.

RAYLEIGH, On the suggested analogy between the conduction of heat and momentum during the turbulent motion of a fluid. Great Britain Advisory Committee for Aeronautics, Reports and Memoranda, No. 497.

REynolds, O., Circumstances which determine the motion of water and the law of resistance in parallel channels. Philosophical Transactions Royal Society of London, $\mathrm{r} 883$, pp. 935-953.

Rogovsky, M. E., Sur la conductibilité Extérieure des Fils d'Argent Plonges dans 1'Eau. Comptes Rendus I36, I903, pp. I391-I393.

Rowse, W. C., Pitot tubes for gas measurement. Transactions American Society of Mechanical Engineers, I9I3, pp. 633-703.

Stanton, T. E., Note on relation between skin friction and surface cooling. Great Britain Advisory Committee for Aeronautics, Technical Report rgr2-I3, pp. 45-47.

Stanton, T. E., and Marshali, D., Heat transmission over surfaces. Great Britain Advisory Committee for Aeronautics. Reports and Memoranda, No. 243.

Stanton, T. E., Booth, H. C., and Marshall, D., On effect of surface roughness of the heat transmitted from hot surfaces to fluids flowing over them with special reference to the case of the gills of an air-cooled engine. Great Britain Advisory Committee for Aeronautics. Report and Memoranda, No. $27 \mathrm{x}$.

Stanton, T. E., and Panneli, J. R., Similarity of motion in relation to the surface friction of fluids. Philosophical Transactions Royal Society of London, 214, I9I4, pp. $199^{-223}$.

TAYLOR, G. I., Conditions at the surface of a hot body exposed to the wind. Great Britain Advisory Committee for Aeronautics. Reports and Memoranda, No. 272.

Etude experimentale sur les radiateurs d'avions. Bulletin de la Section Technique de 1'Aeronautique Militaire, Aout, I9I8.

Experiments on aeroplane radiators. Eiffel laboratory, September, I9I7.

Radiators for airplane engines. Aviation and Aeronautical Engineering, December I5, I917, pp. 686-687.

Radiators for water-cooled engines. Great Britain Advisory Committee for Aeronautics. Reports and Memoranda, No. 407.

Slipstream corrections in performance computation. National Advisory Committee for Aeronautics. Technical Report No. $7 \mathrm{I}$.

Turbulence. National Advisory Committee for Aeronautics. Technical Report No. 106. 

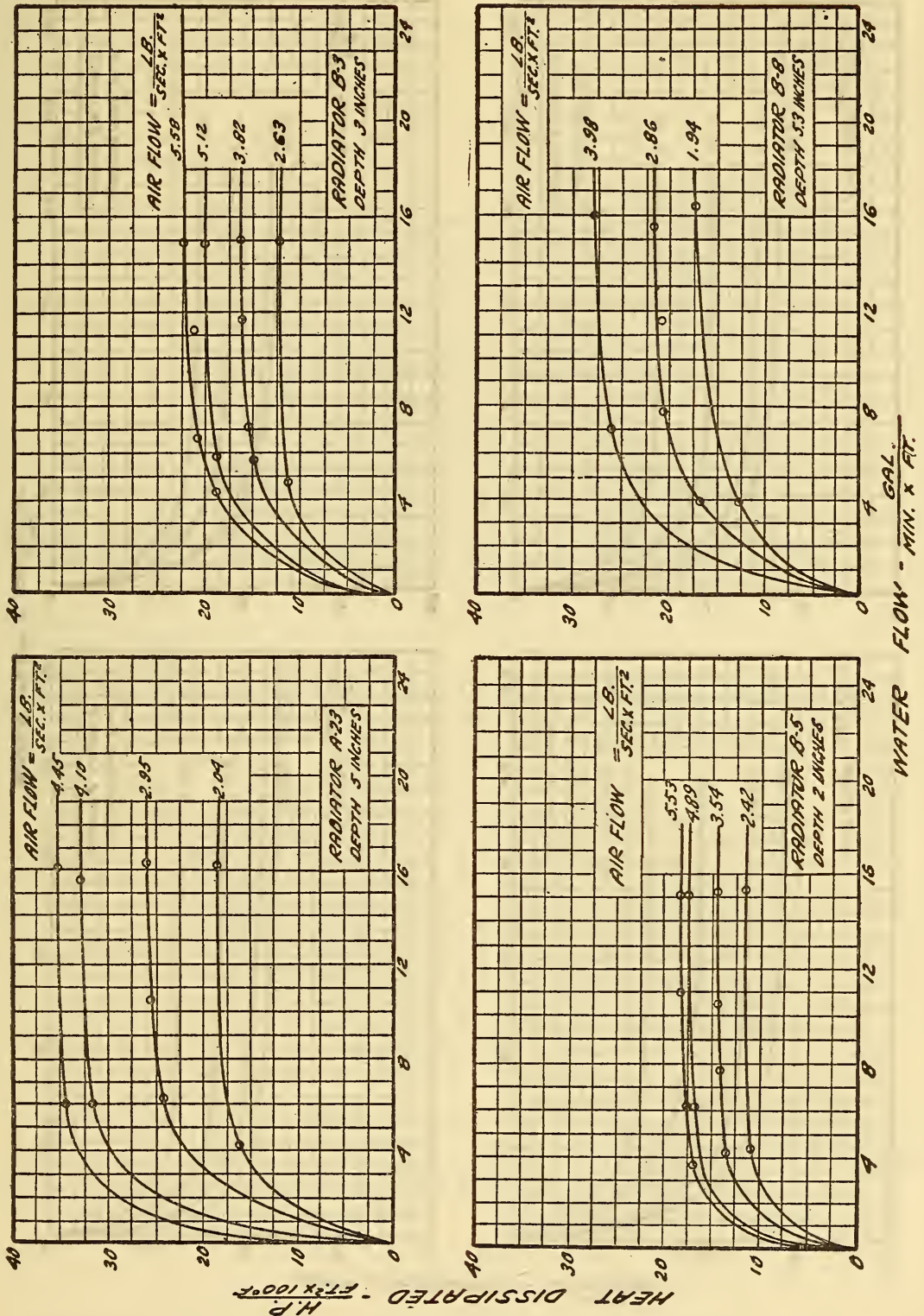

FIGS. I28-3I.-Heat dissipation as a function of rate of water flow in a radiator core

Data from which the figures were drawn will be found in Table 25, under the respective core listings $\mathrm{A}-23, \mathrm{~B}-3, \mathrm{~B}-5, \mathrm{~B}-8$. Conclusions drawn from the curves are discussed in Art. 56 , Table ro. 

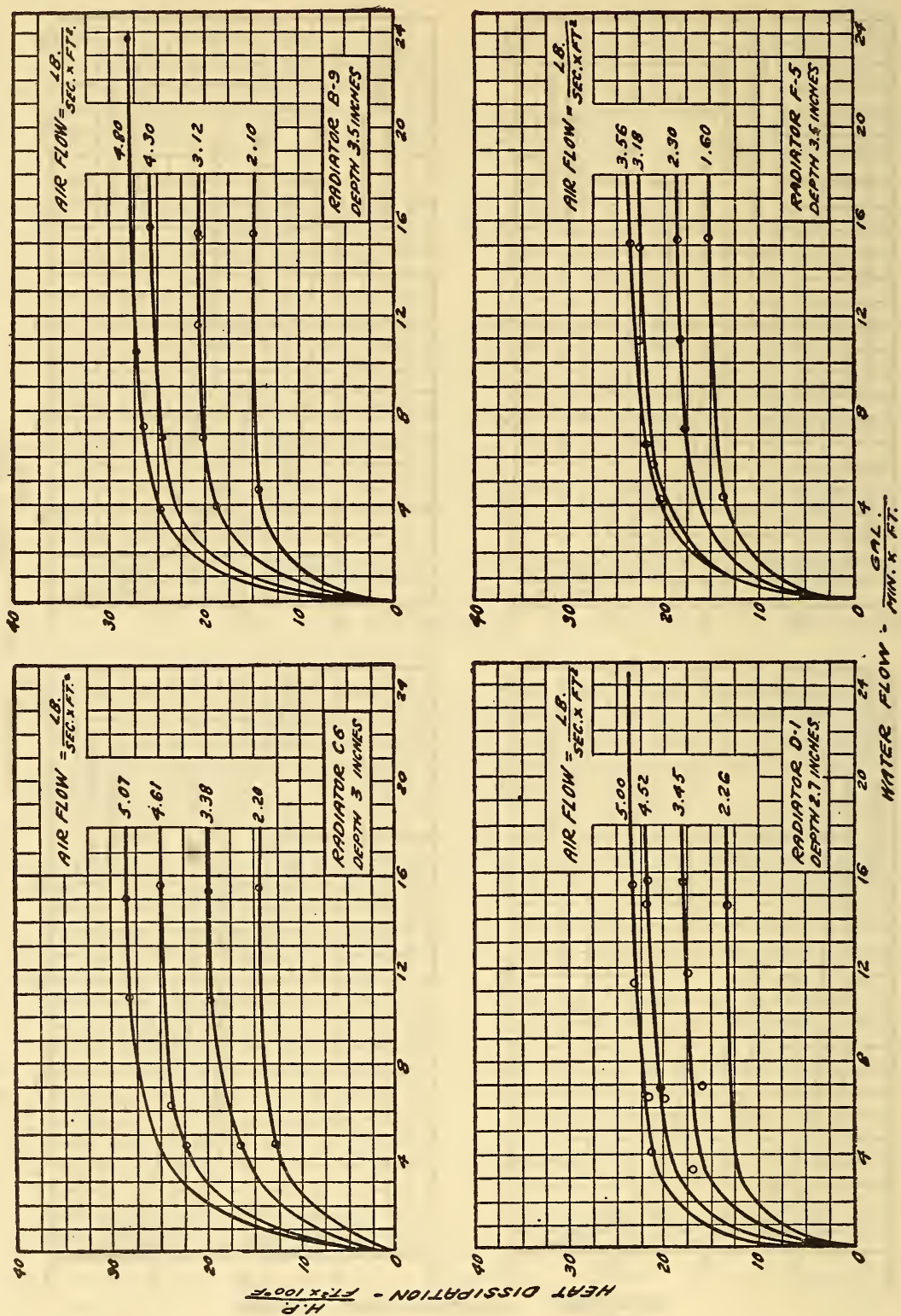

FIGS. I32-135.- Heat dissipation as a function of rate of water flow in a radiator core Data from which the figures were drawn will be found in Table 25, under the respective core listings B-9, C-6, D-r, F-5. Conclusions drawn from the curves are discussed in Art. 56, Table Io. 


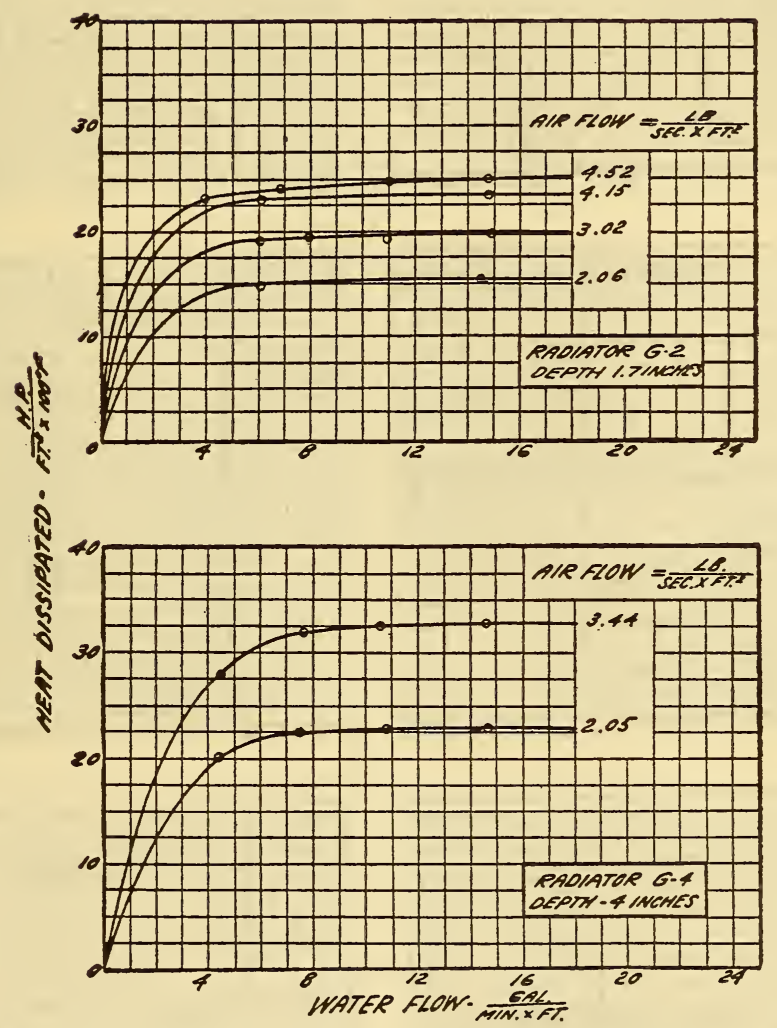

FIGS. 136-I37.-Heat dissipation as a function of rate of water flow in a radiator core

Data from which the figures were drawn will be found in Table 25 , under the respective core listings $\mathrm{G}-2, \mathrm{G}-4$. Conclusions drawn from the curves are discussed in Art. 56, Table ro 

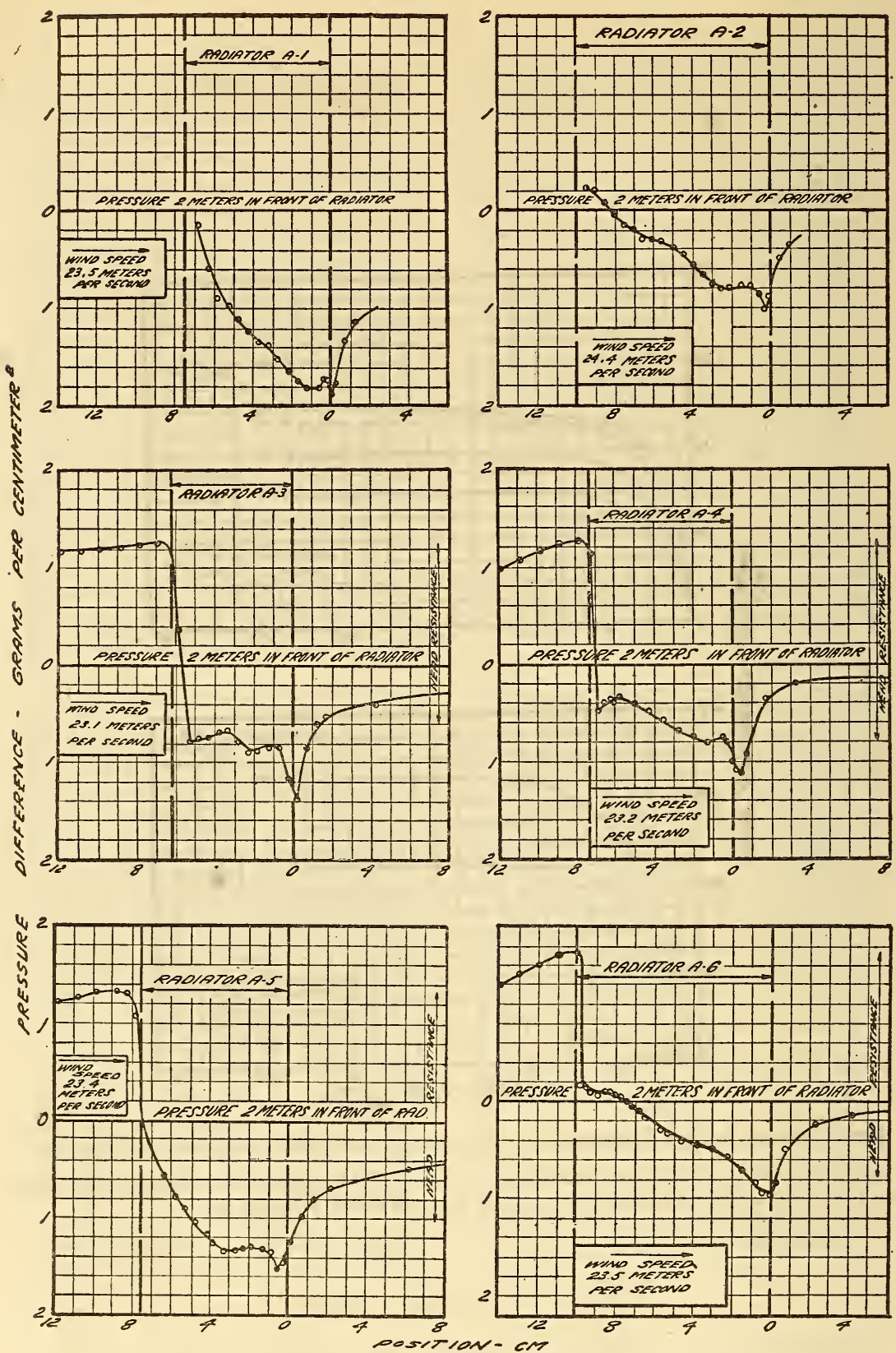

FIG. I38.-Air flow data for six square cell cores $(A-I$ to $A-6)$

By mapping the pressure gradient in the air tubes as described in Arts. $15-17$, the rate of air flow was determined. The graphic record of original observations is reproduced in lieu of tabulating the data. The wind speed is that in the large "open" wind tunnel some distance in front of the radiator 

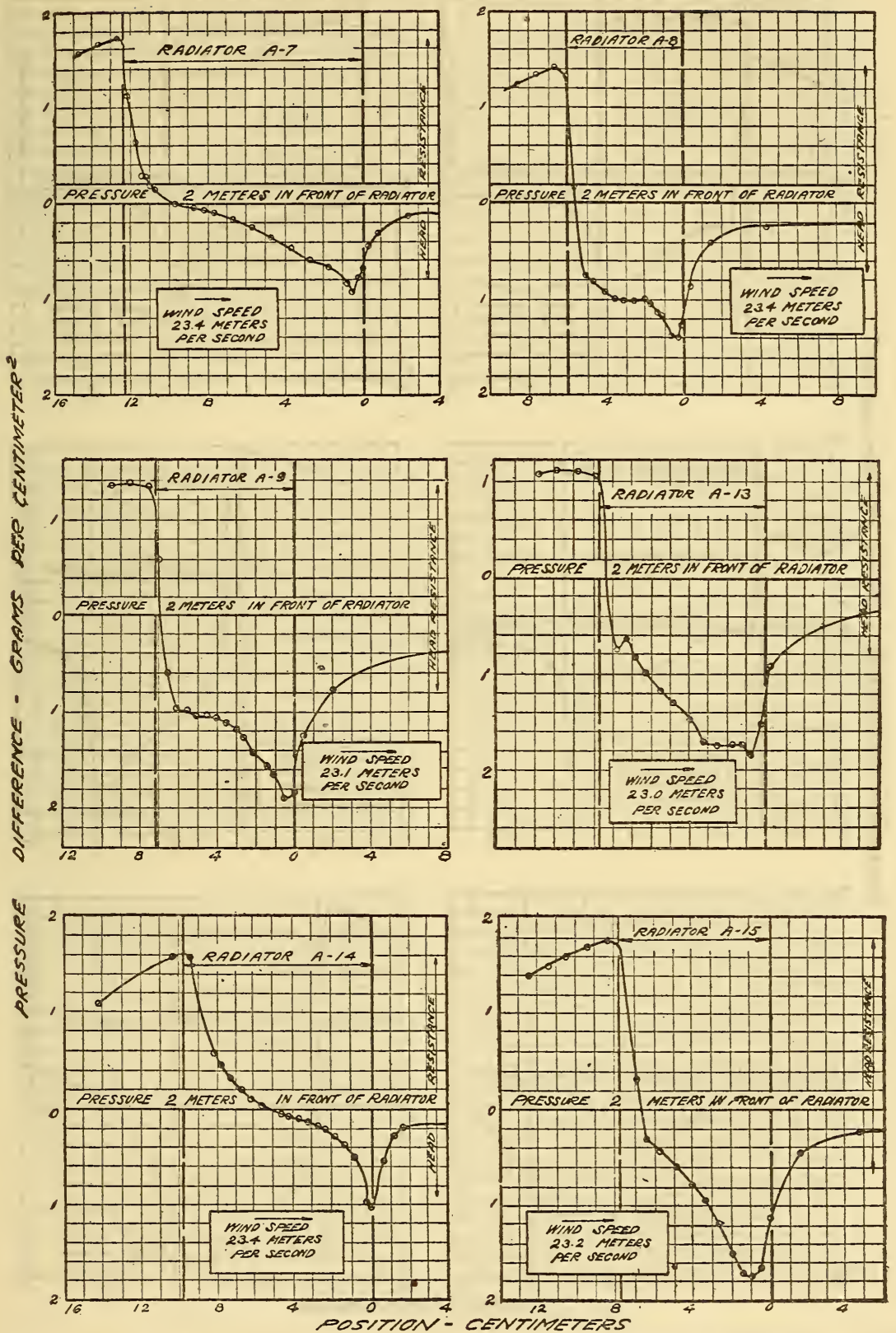

FIG. I39.-Air flow data for six square cell cores $\left(A-7,8,9, I_{3}, I_{4}, I_{5}\right)$

By mapping the pressure gradient in the air tubes as described in Arts. $\mathrm{I}_{5}^{-1} \mathrm{I}_{7}$, the rate of air flow was determined. The graphic record of original observations is reproduced in lieu of tabulating the data. The wind speed is that in the large "open" wind tunnel some distance in front of the radiator 


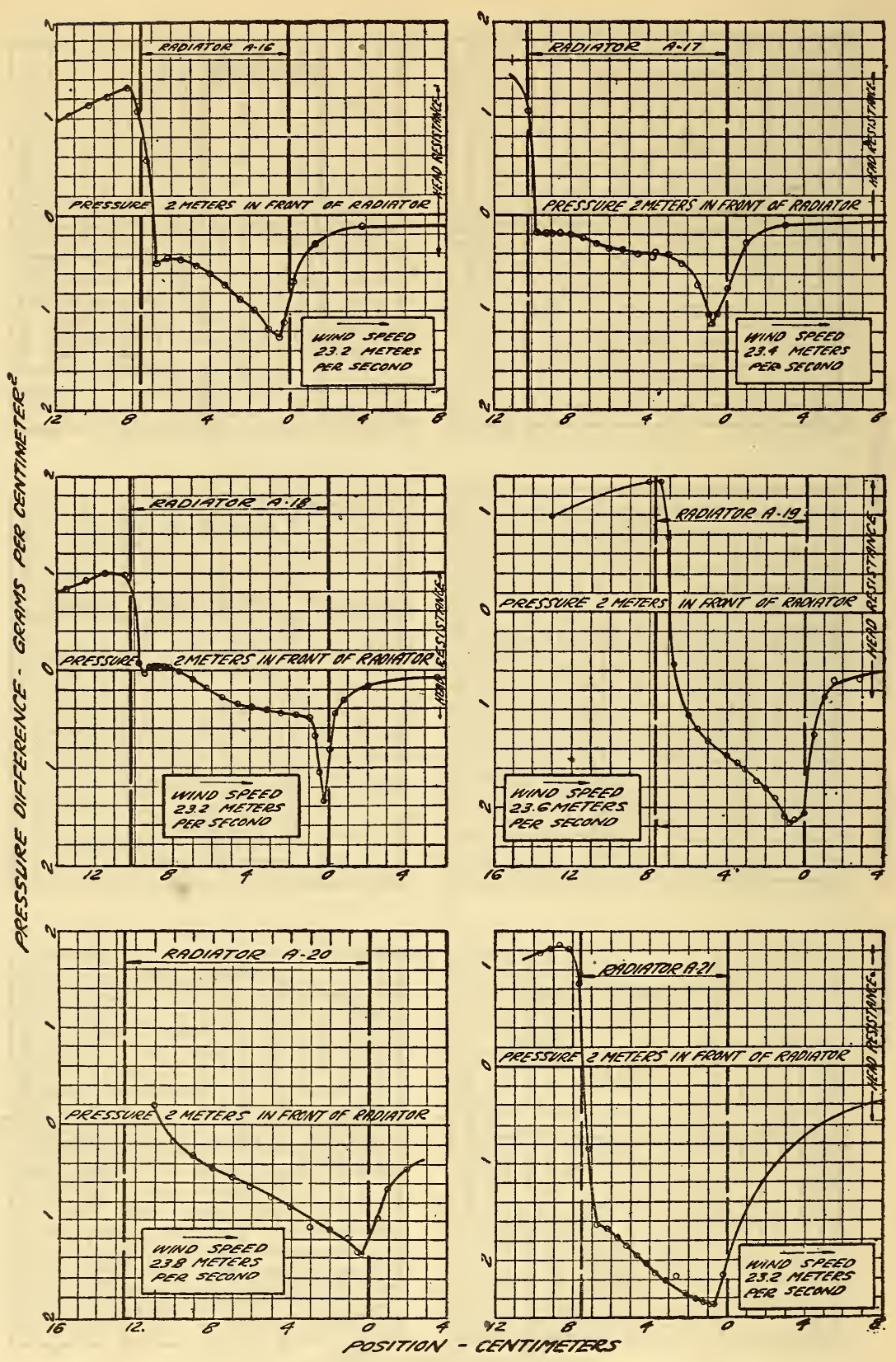

FIG. I40.- Air flow data for six cellular cores $(A-I 6$ to $A-2 I)$ with air tubes of rectangular (nearly square) cross section

By mapping the pressure gradient in the air tubes as described in Arts. $15^{-17}$, the rate of air flow was determined. The graphic record of original observations is reproduced in lieu of tabulating the data. The wind speed is that in the large "open" wind tunnel some distance in front of the radiator 

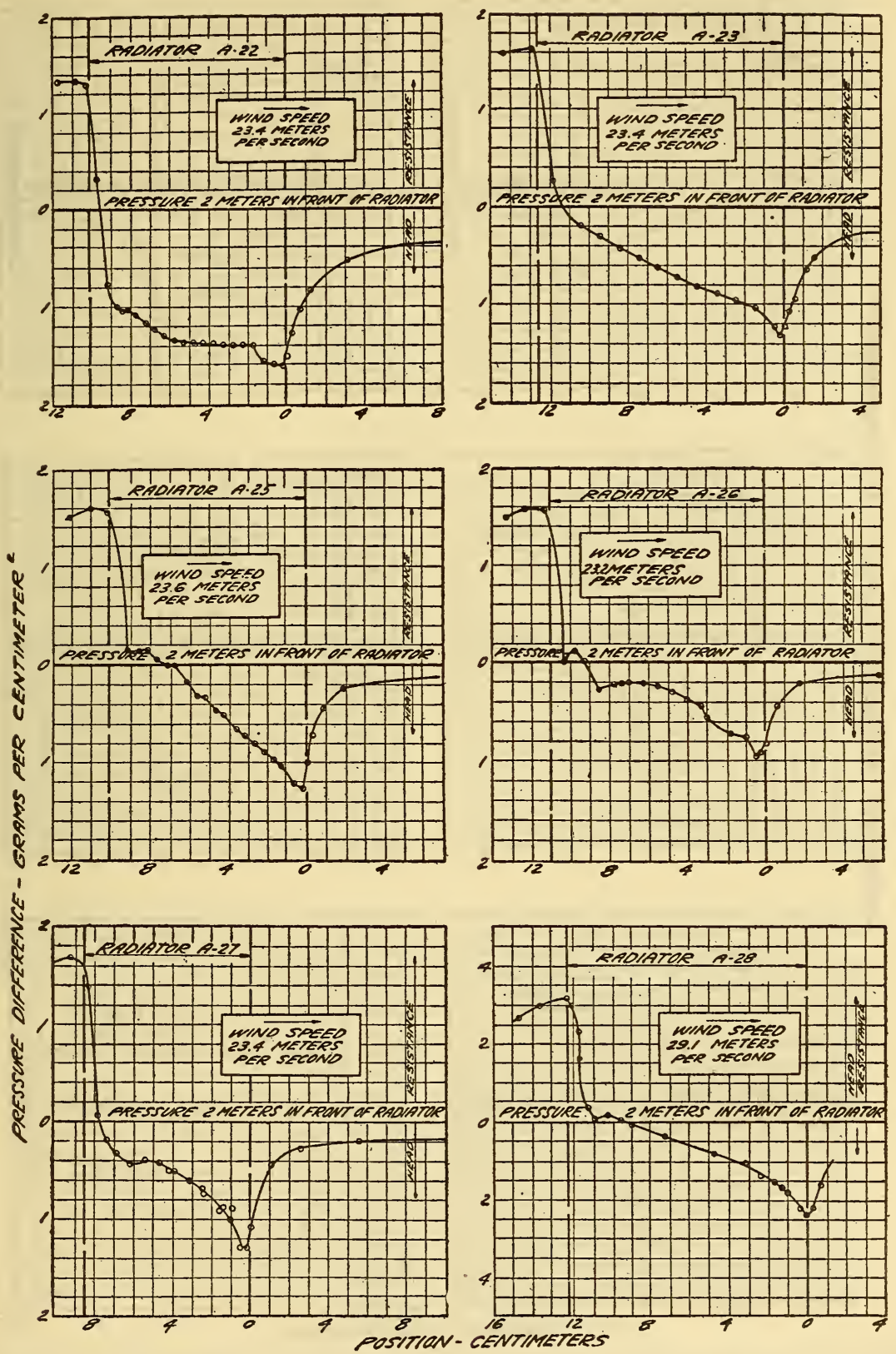

FIG. I4I.--Air flow data for six square cell cores $(A-22$ to $A-28)$

By mapping the pressure gradient in the air tubes as described in Arts. ${ }^{5}-17$, the rate of air flow was determined. The graphic record of original observations is reproduced in lieu of tabulating the data. The wind speed is that in the large "open" wind tunnel some distance in front of the radiator 


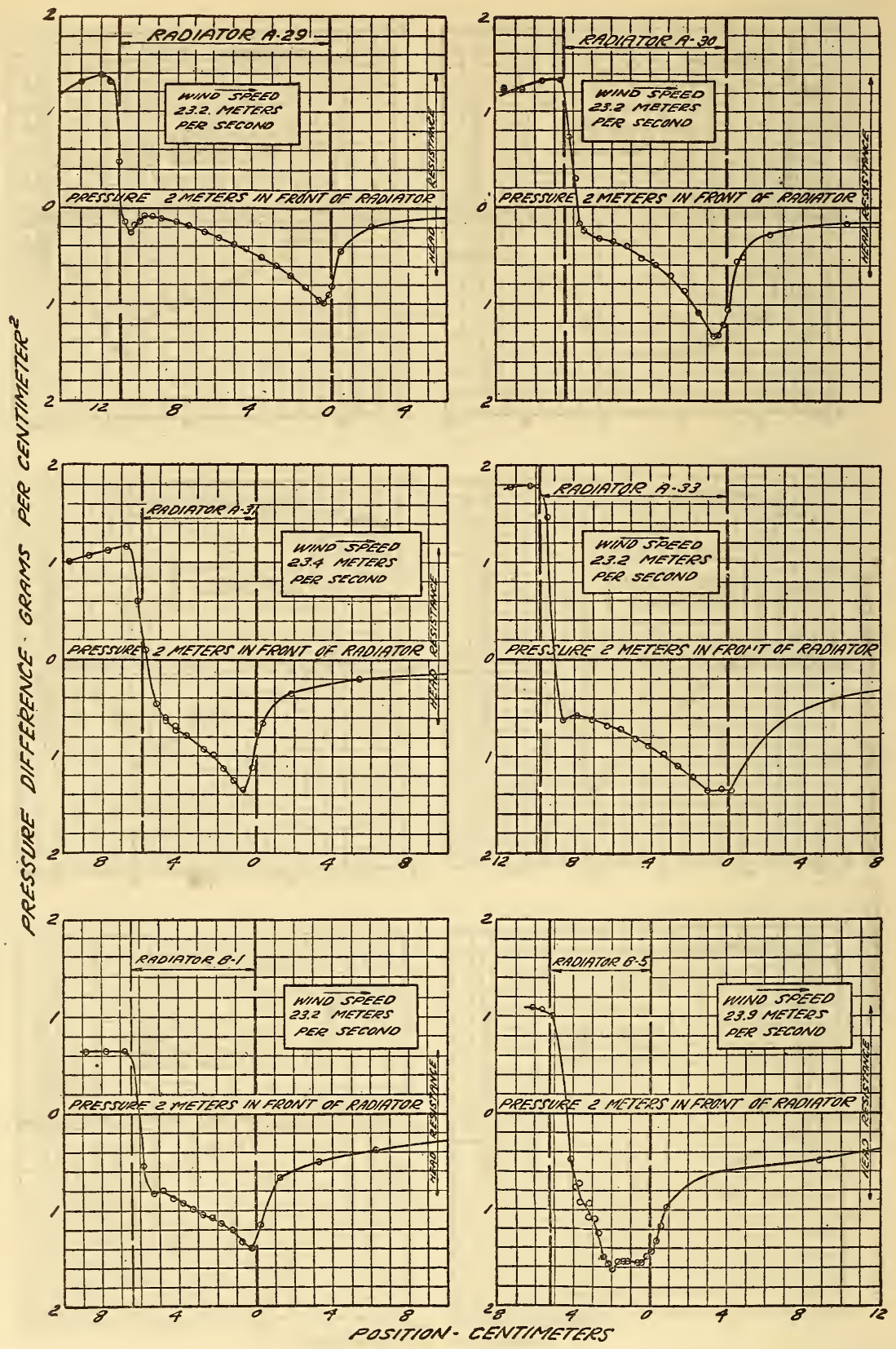

Fig. I42.-Air flow data for four square cell cores $(A-20$ to $A-33)$ and two hexagonal cell cores $(B-I, B-5)$, the first of simple type and the second with a lattice of turbulence vanes

By mapping the pressure gradient in the air tubes as described in Arts. $15-17$, the rate of air flow was determined. The graphic record of original observations is reproduced in lieu of tabulating the data. The wind speed is that in the large "open" wind tunnel some distance in f ront of the radiator 

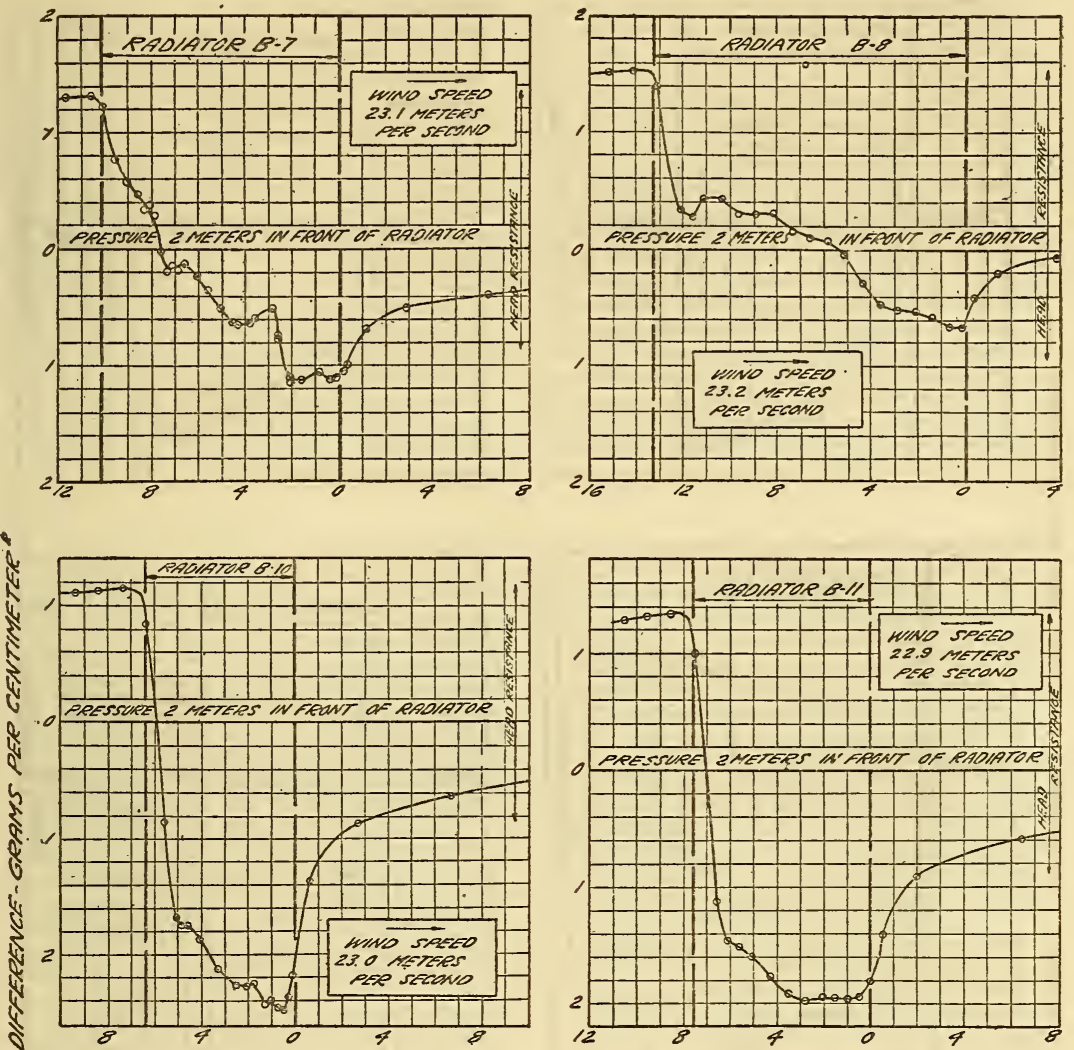

है
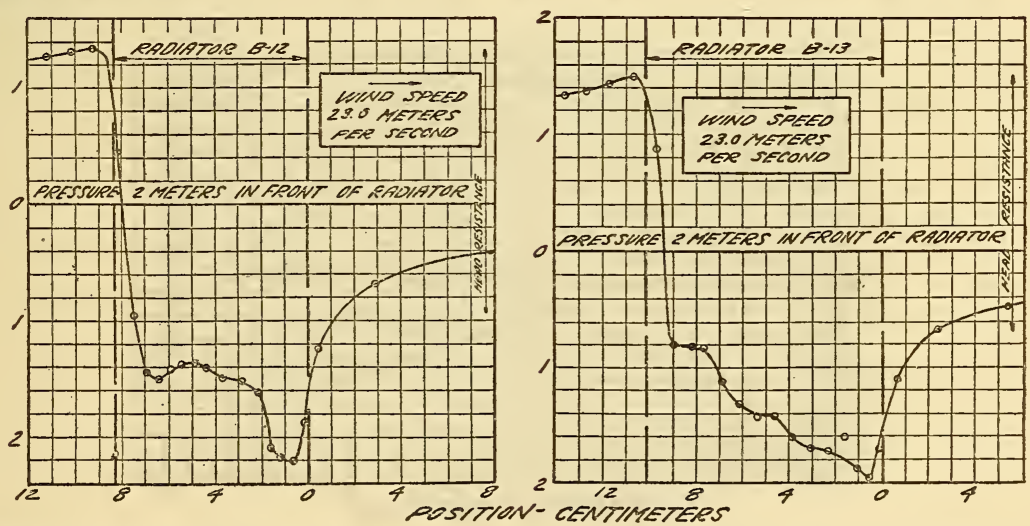

FIG. I43.-Air flow data for six hexagonal cell cores (B-7 to B-I3), the air tube walls being latticed or provided with turbulence vanes

By mapping the pressure gradient in the air tubes as described in Arts. $15^{-17}$, the rate of air flow was determined. The graphic record of original observations is reproduced in lieu of tabulating the data. The wind speed is that in the large "open" wind tunnel some distance in front of the radiator 

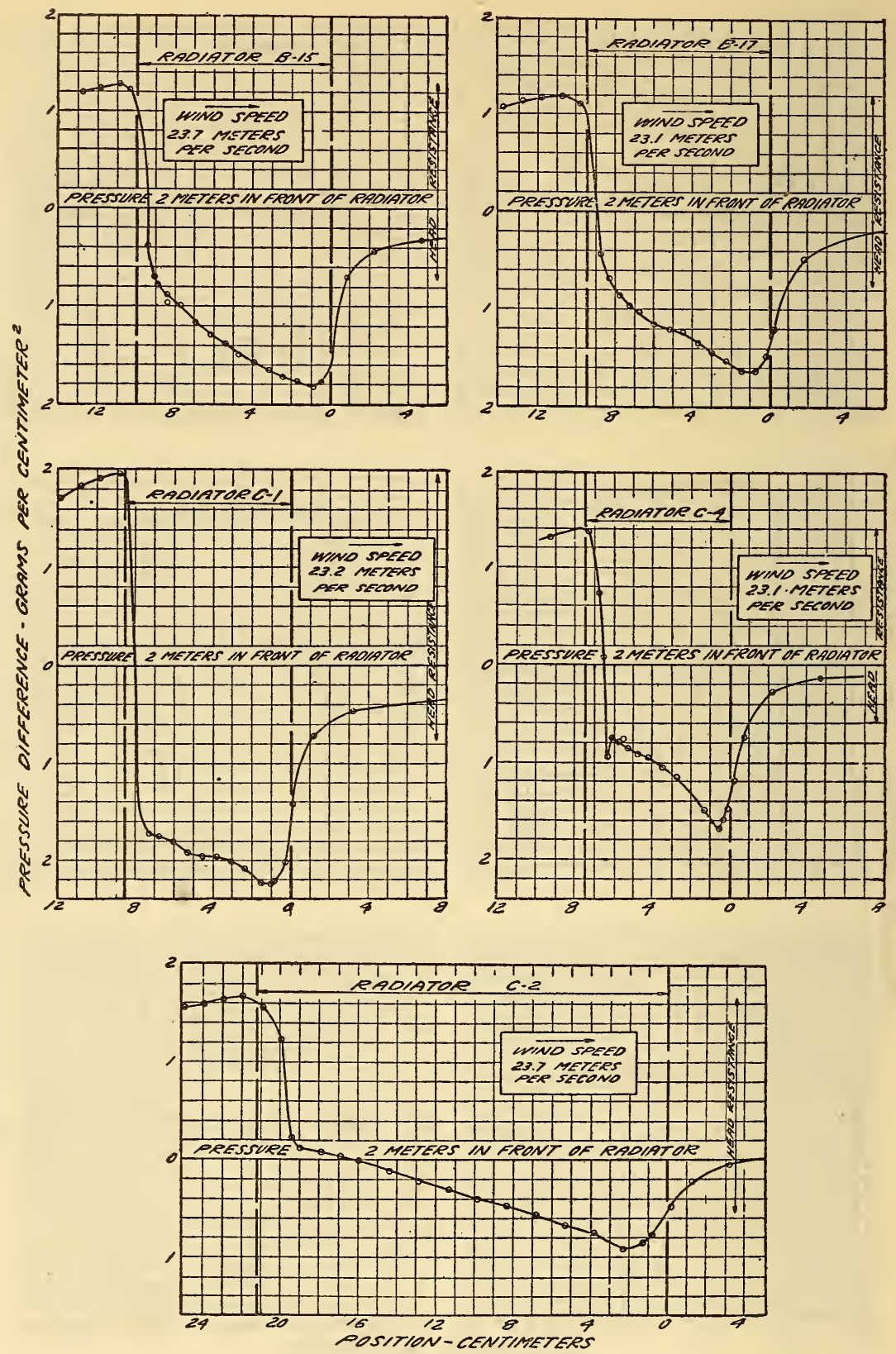

FIG. I44.-Air flow data for two hexagonal cell cores $(B-I 5, B-I 7)$ of simple type without any turbulence vanes or other distorting elements, and of three other cellular cores $(C-I$, $C-4, C-2)$ of irregular form, illustrated in Figs. 88, 9I, 89. C-2 has plain circular air tubes and it will be noted that the pressure gradient curve is very smooth and regular

By mapping the pressure gradient in the air tubes as described in Arts. 15-17, the rate of air flow was determined. The graphic record of original observations is reproduced in lieu of tabulating the data. The wind speed is that in the large "open" wind tunnel some distance in front of the radiator 


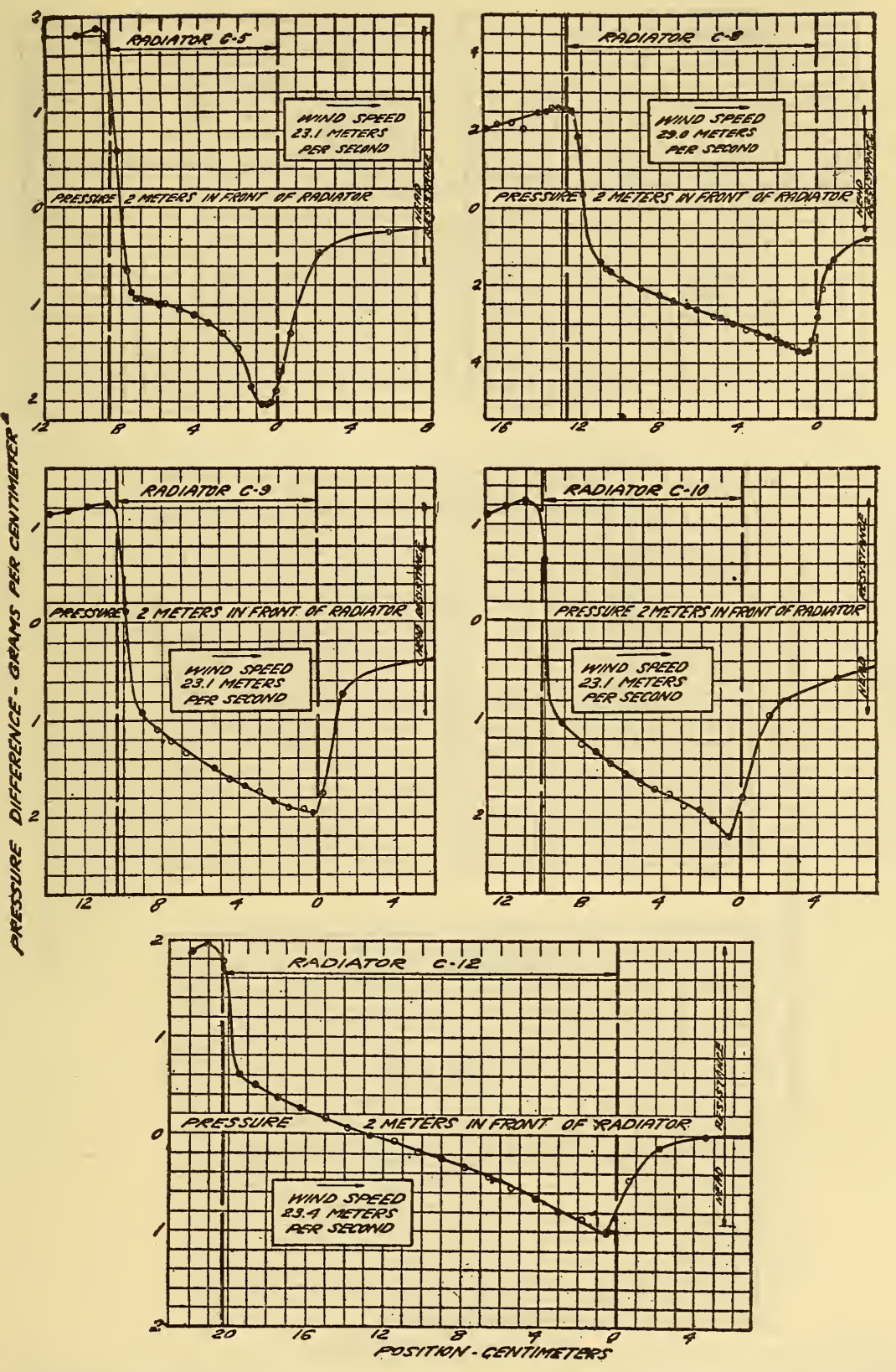

Fig. 145.-Air flow data for five circular cell cores. $C-5$ is of peculiar construction illustrated in Fig. 92. Air flow in the smooth circular tubes is modified by the entrance condition incident to having the "cushion" shaped cell adjacent to it. C-8 to C-I2 are circular tube cores with the ends of the air tubes pressed'into hexagons for joining together

By mapping the pressure gradient in the air tubes as described in Arts. $15^{-17}$, the rate of air flow was determined. The graphic record of original observations is reproduced in lieu of tabulating the data. The wind speed is that in the large "open" wind tunnel some distance in front of the radiator 

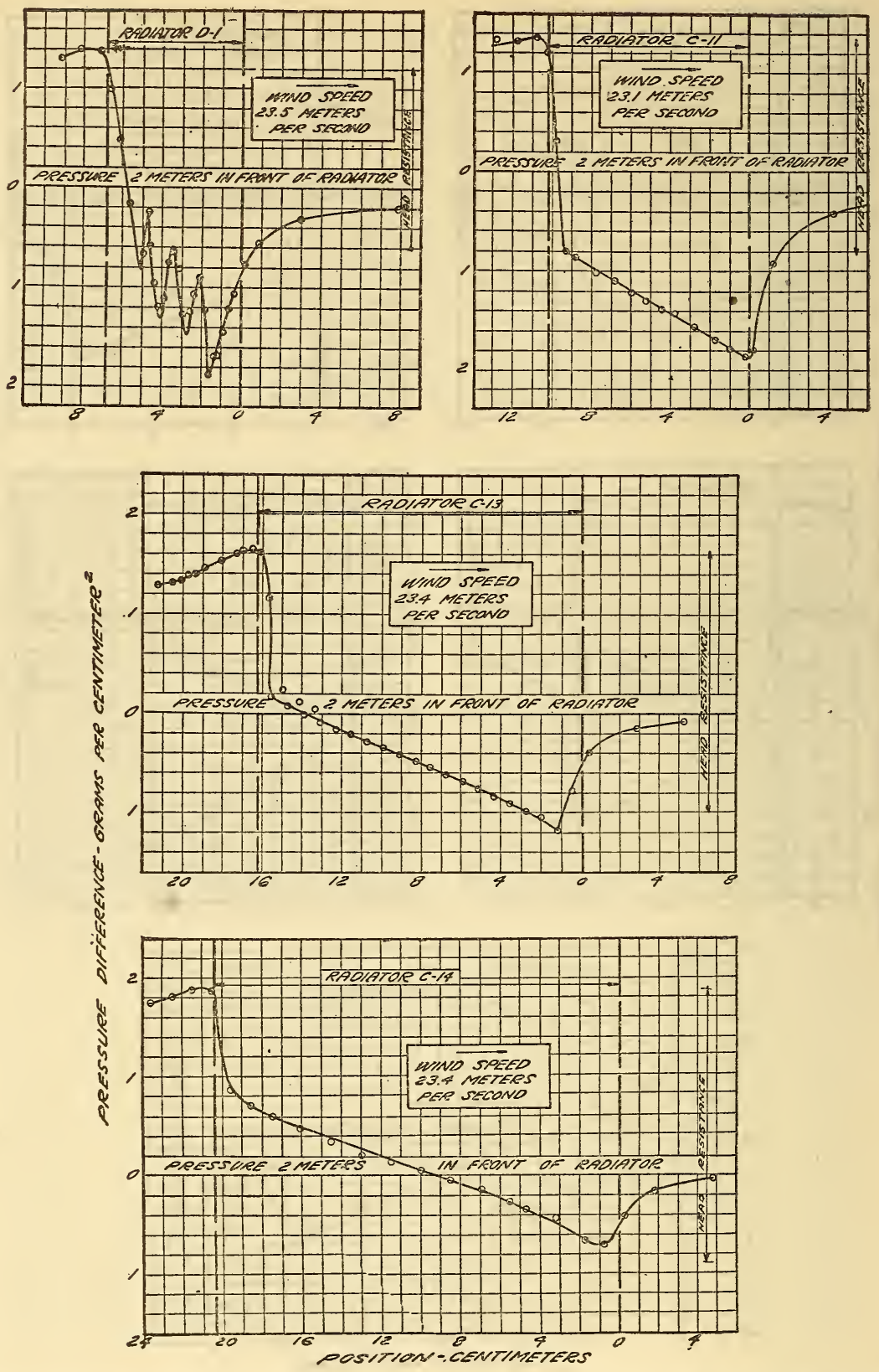

FIg. 146.-Air flow data for three circular cell cores $(C-I I, I 3, I 4)$ in which the circular tubes are pressed to hexagonis at the ends, and for an irregular core of construction shown in Figs. IOZ and IO3. The oscillating character of the pressure gradient curve is due to the dimples shown in Fig. 103

By mapping the pressure gradient in the air tubes as described in Arts. $15^{-17}$, the rate of air flow was determined. The graphic record of original observations is reproduced in lieu of tabulating the data. The wind speed is that in the large "open" wind tunnel some distance in front of the radiator 


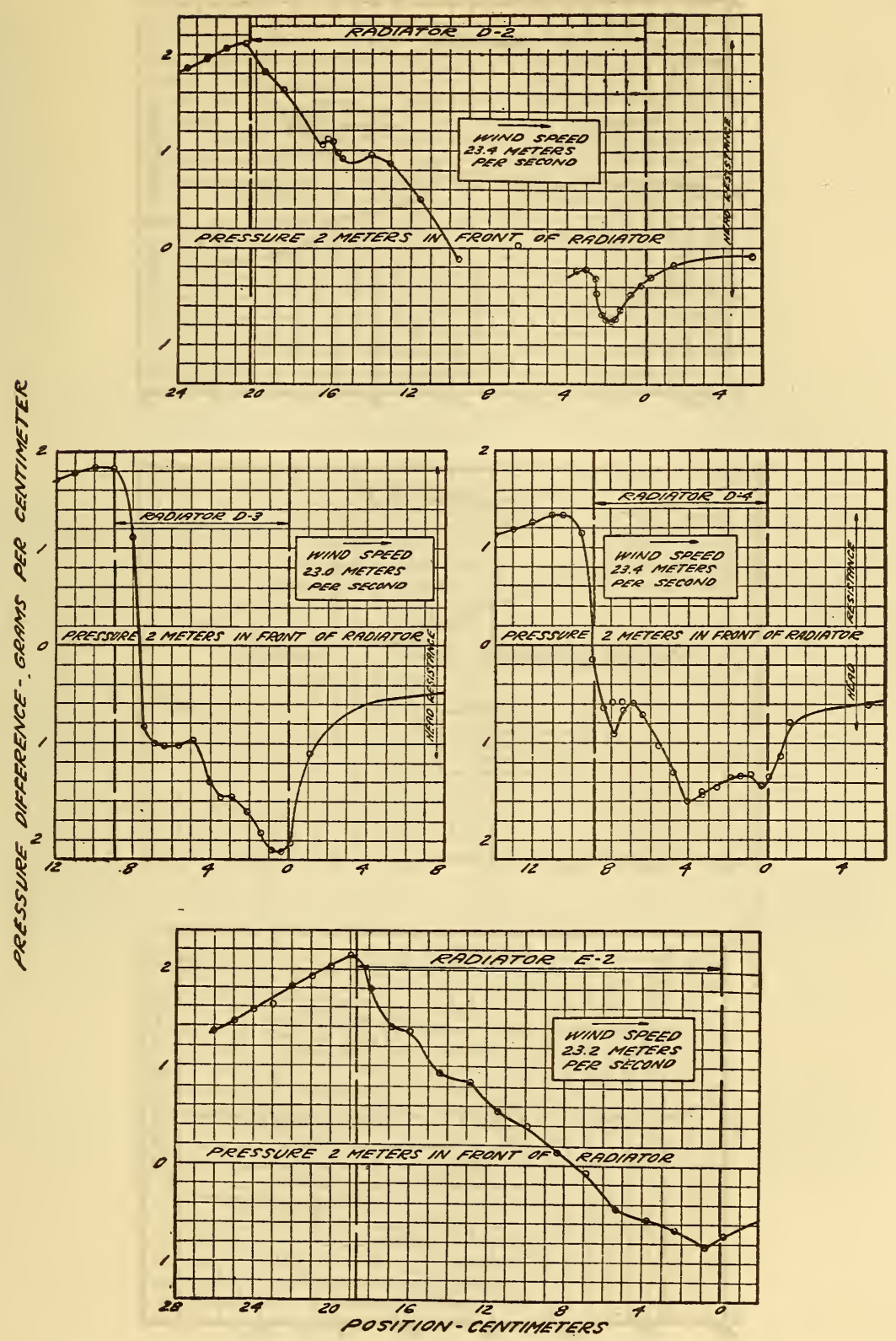

FIG. 147.-Air flow data for miscellaneous cores of construction shown in the heading of Table $20(p .368)$. E-2 is a flat plate core with perforated water tubes giving irregularities in the air flow

By mapping the pressure gradient in the air tubes as described in Arts. $15^{-17} 7$, the rate of air flow was determined. The graphic record of original observations is reproduced in lieu of tabulating the data. The wind speed is that in the large "open" wind tunnel some distance in front of the radiator $75013^{\circ}-22-12$ 

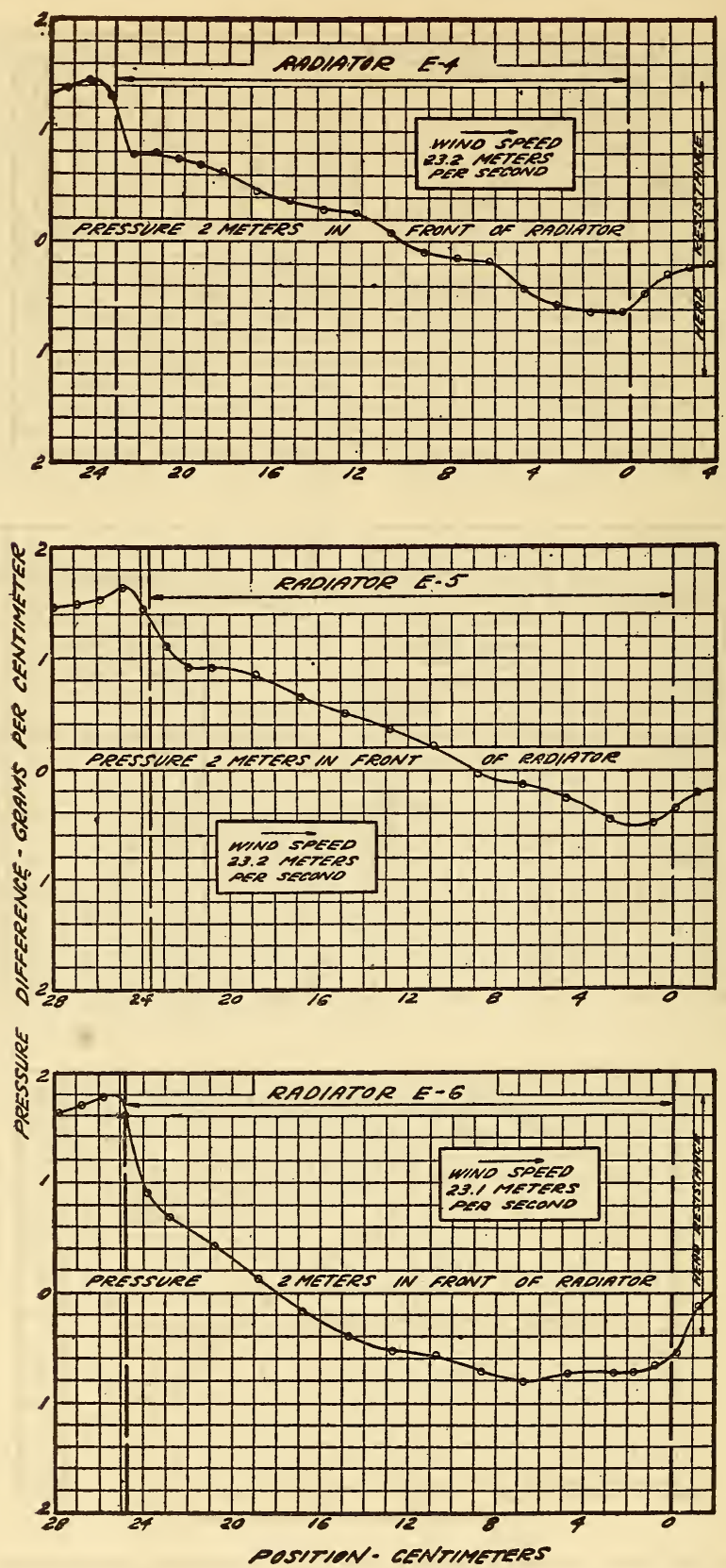

FIG. I48.-Air flow data for two perforated plate cores $(E-4, E-5)$ and flat plate core E-6

By mapping the pressure gradient in the air tubes as described in Arts. $15-17$, the rate of air flow was determined. The graphic record of original observations is reproduced in lieu of tabulating the data. The wind speed is that in the large "open" wind tunnel some distance in front of the radiator 

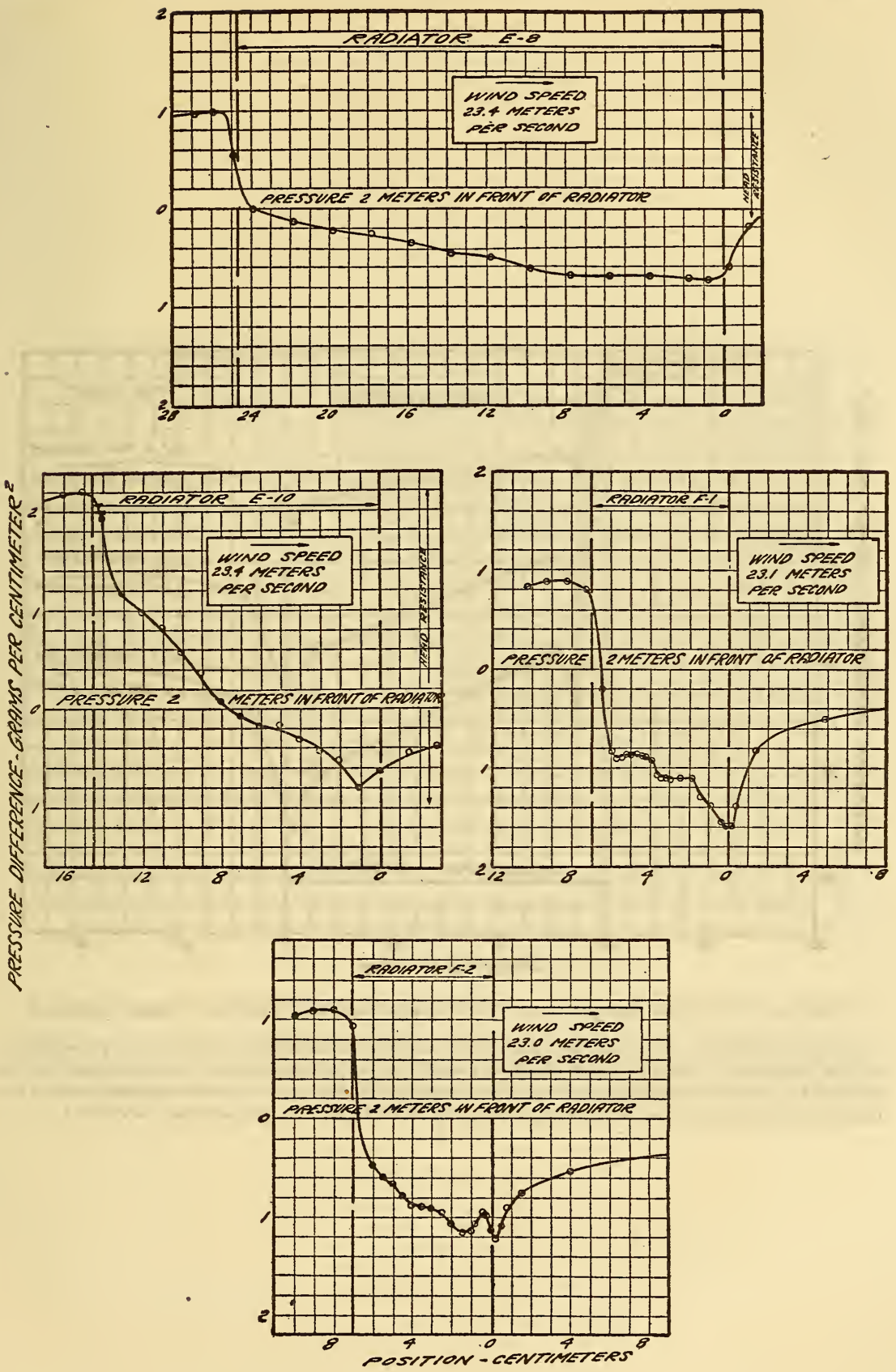

FIG. I49.-Air flow data for flat plate core E-8, corrugated plate core E-Io, and two fin and tube cores $(F-I, F-2)$

By mapping the pressure gradient in the air tubes as described in Arts. $15^{-17}$, the rate of air flow was determined. The graphic record of original observations is reproduced in lieu of tabulating the data. The wind speed is that in the large "open" wind tunnel some distance in front of the radiator 


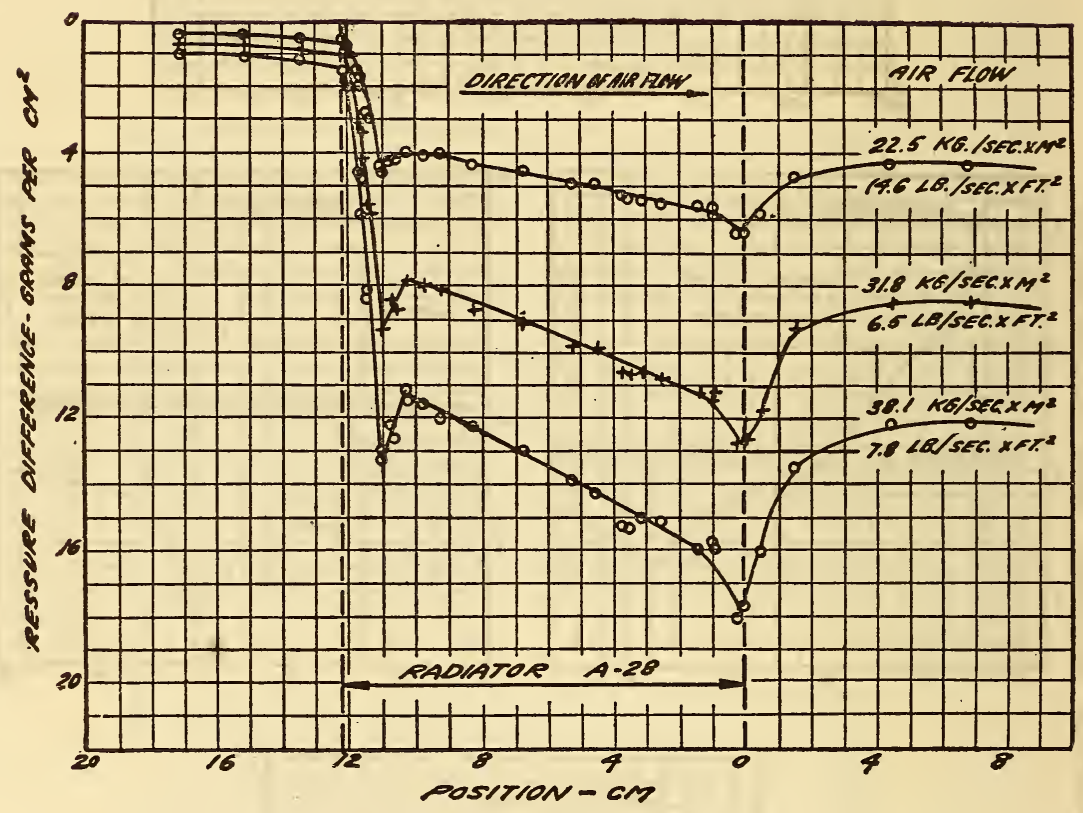

Fig. I 50.-A ir flow data for core A-28 (square cell) in closed (or "steam") tunne?

This figure illustrates the method of using the pressure tube as described in Arts. 15-17. Three rates of air flow, measured by the pitot grid in the closed tunnel, give the calibration of the "pressure tube" in this core and by comparing the pressure drop curve for core $\mathrm{A}-28$ in Fig. I4I with an interpolated curve of the family defined by the above three curves, a computation of the air flow constant may be effected 


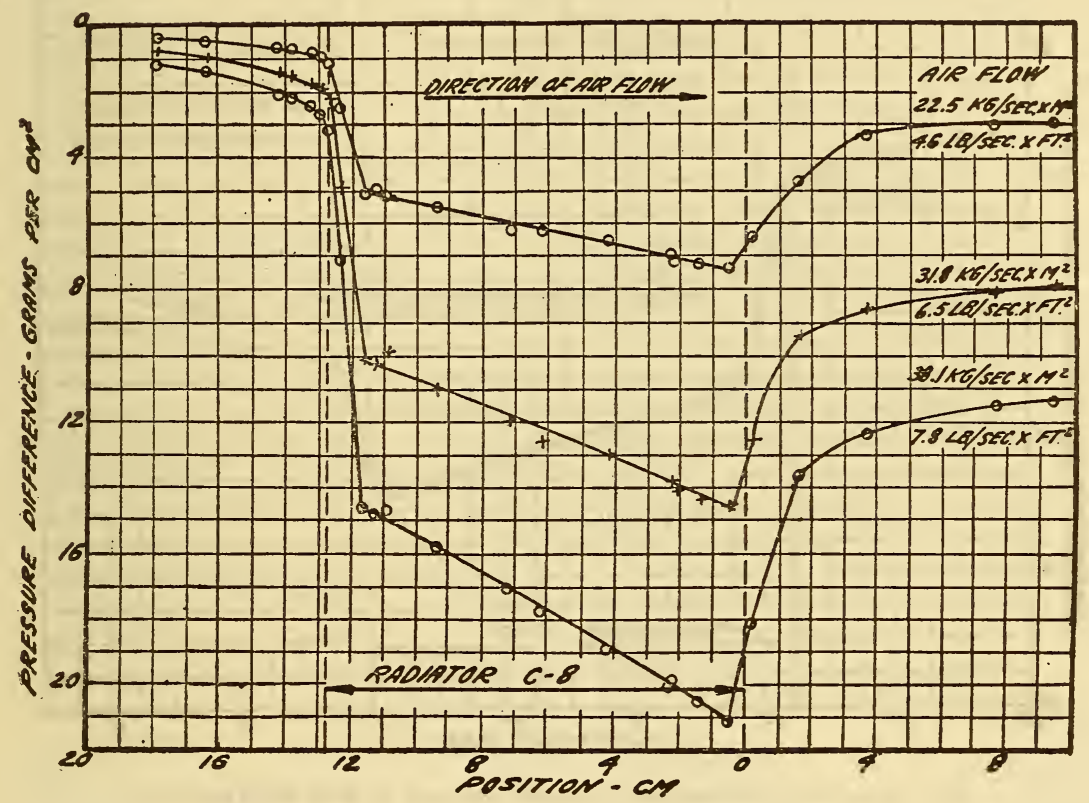

FIG. I5I.-Air flow data for a circular cell core in the closed (or "steam") tunnel

Data used exactly as described above for Fig. 150. The figure is introduced to show how very regular is the pressure drop through a simple cylindrical air tube. Curves of this same nature, at three or more rates of air flow, were required for each core, being combined with Figs. $138-149$ to evaluate the air flow constants 


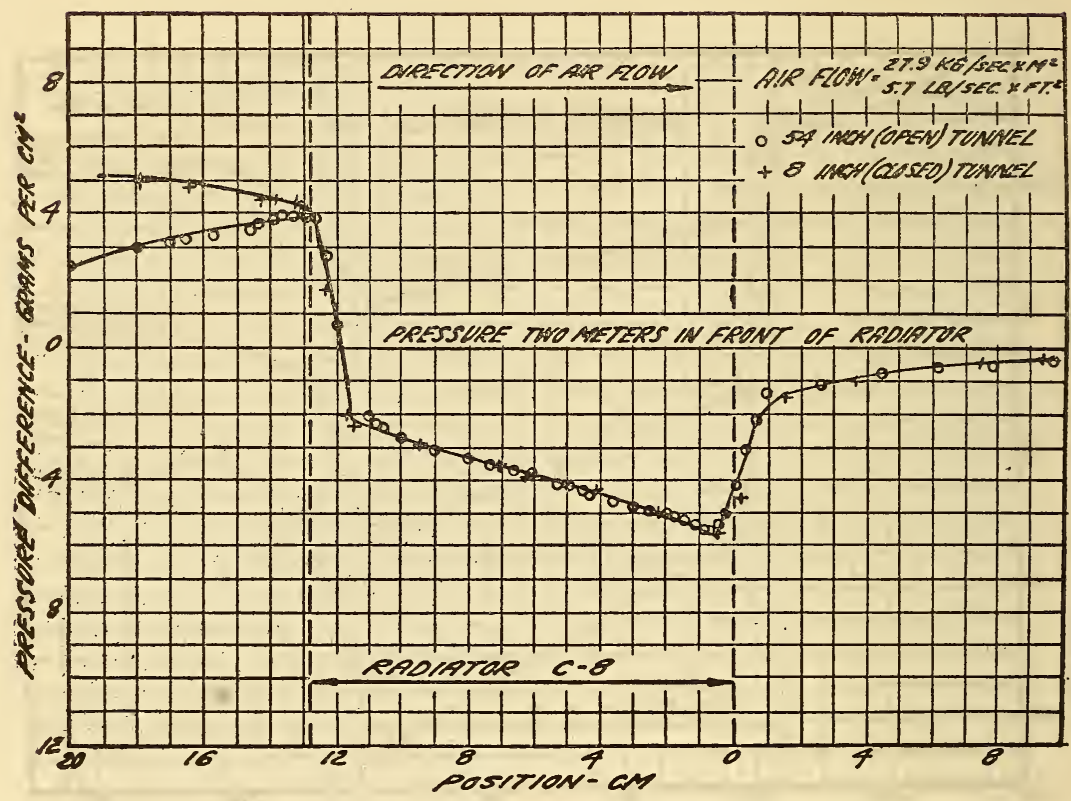

Fig. 152.-Air flow data for a circular cell core in both wind tunnels

A laboratory interpolation of curves usually interpolated by graphic or analytic means. Having mapped pressure gradient in the closed tunnel for a given air flow, the core was mounted in the open tunnel and the wind speed adjusted to give the same pressure drop at some point, and as the curves show this means coincidence at all points (except immediately in front of core). This procedure furnished an excellent test of the validity of the assumptions on which air-flow-constant determinations were made.

WASHINGTON, June 2 I, $192 \mathrm{I}$. 


\section{INDEX}

Air flow (Chapter

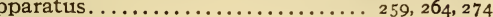

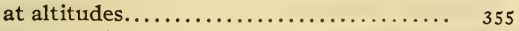
computation. ................... 262,27I

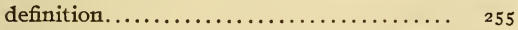

density of air................. 257,263, 275

depth of core...................... 332

empirical equation. .............. 276, 277 experimental data for cores (in graphic

form, Figs. $\left.\mathrm{r}_{3} 8-\mathrm{r}_{52}\right) \ldots \ldots \ldots \ldots \ldots \ldots 4^{\mathrm{r} 2-426}$

fin and tube types................... 344

flat plate types. . . . . . . . . . . . . . . . . 344

geometrical characteristics........ 276, 330-349

head resistance.................. $28 \mathrm{I}$

heat dissipation $\ldots \ldots \ldots \ldots \ldots \ldots \ldots 3$ I6, 3 I 8,356

instruments. See Apparatus.

measurements. See Measurements.

nose radiator................... $\quad 279$

obstructed positions.............. $278-28$ r

position of radiator. . . . . . . . . .

pressure difference............. 258, 272-275

slip stream. . . . . . . . . . $\ldots \ldots \ldots \ldots$ 280, 28r, 353

speed of flight .................... 275

stream-line casing ................ 348, 349

stream-line flow............... 258, 394, 399

surface, condition of . . . . . . . . . 276, 277

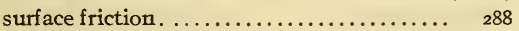

temperature difference.............. 290, 29I

turbulence, effect on air flow. ......... 340, 34r

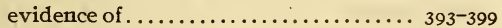

unobstructed positions ........... 275-279, 353

values ('Table 21 ).................. 372-376

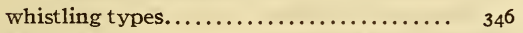

wing positions.............. 279, 280, 345, 353

yaw.......................... 278, 354

Air-flow constant, computation......... 27 $1-275$

definition........................ ${ }_{255}$

empirical equation................. $276-278$

nose position................... $\quad 279$

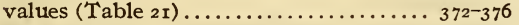

wing position.................... 280

Air tubes, air-flow constant........... 276-278

form and dimensions............... 339

measurement. (See Measurements.)

smoothness of surface............. 332

surface friction................ 277, 287, 289

Altitude, atmospheric conditions and their

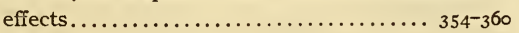

Anemometer, hot wire.............. 268

Apparatus. See Measurements.

air flow, open wind tunnel........ 267-270, 275 reduced pressure tunnel.............. ${ }_{26}$ steam tunnel...................... 259 gages..................... 262, 268, 274 head resistance............... 282, 283, 296, 299

heat transfer, reduced pressure tunnel..... 308 single tube...................... 324 steam tunnel.................... 303
Apparatus, resistance thermorneter.... . Page. 264,308 thermocouples. See Thermocouples.

water pump......................... 310

Balance, aerodynamic............... 282, 283

Boiling point of water at altitudes ........ 355

Calibrations, head resistance balance........ ${ }_{2} 8_{3}$

pitot grid....................... 260-262

thermocouples.................... 309

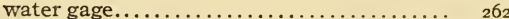

Calorimetric measurements. See Measurements.

Carrier, W. H...................... ${ }_{26} 66$

Cellular radiators. See Types of core.

Circular cells. See Types of core.

Comparison of steam and water.......... 313,314

Condensation of steam.............. 305

Conductivity, thermal, direct cooling surface. $3 \mathrm{I} 4$

$315,330,402$

indirect cooling surface........ 3 I 4,3 I $5,327-330$

Contact, thermal. See Thermal contact.

Cooling capacity. See Heat dissipation.

Cooling coefficient. See Heat dissipation

coefficient.

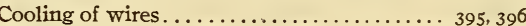

Cooling surface, heat transfer....... 303, 322, 33 I

indirect cooling surface............. 327

effectiveness.............. 328-330, 332, 333

derivation of equation. . . . . . . . . . 404-406

proportions for fins. . . . . . . . . . 332, 333

measurements.................... 352

metal. See Conductivity, thermal.

nature of surface............... 331, 332

air flow............................ 277

head resistance. . . . . . . . . . . 288, 289, 293

heat transfer................... 323-326

wing and strut surface. . . . . . . . . 347,348

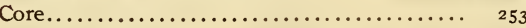

Corrugated plates................... ${ }_{346}$

Definitions....................... 253-257

Density of air, air flow................ $\quad{ }_{275}$

at altitudes........................ 355

head resistance................. 29I, 292

heat dissipation. ....................... 3I5, 3 I 6

humidity correction. . . . . . . . . . . . . $26_{3}-26_{5}$

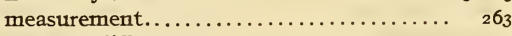

pressure difference................ 272, 273

properties of radiators at altitudes....... $357,35^{8}$ reductions to standard density, air flow... 4 or

head resistance................. 285,286

"standard"........................ ${ }_{28} 8_{5}$

surface friction................... 258, 287

Depth of core, air flow.............. 276, 333

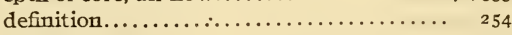

figure of merit.................... 333

head resistance.................. 293, 337

heat dissipation. ............... 317, 323, 333

optimum........................338, 339 


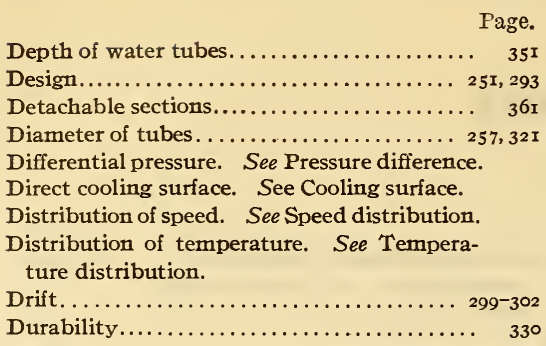

Ease of repair.................. 25I, 315, 330

Eddy resistance. See Head resistance.

definition...................... ${ }_{282}$

depth of core.................... 333

fin and tube types. . . . . . . . . . . . . . . . 344

irregular tubes.................... 339

speed........................ $29 \mathrm{I}$

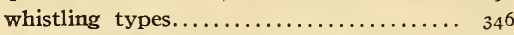

Effectiveness of indirect cooling surface..... 323 derivation.................... 404

Empirical equations, air flow......... 276, 277

head resistance. . . . . . . . . . . . . . . 292

heat transfer................... $3{ }^{17}$

performance........................ 356

Entrance air temperature. . 306, 3 II, 315, 319, 402, 403

Expansion of air in radiator tube......... 290

Experimental procedure. See Measurements.

Fifty-four inch wind tunnel. See Wind tunnels, open tunnel.

Figure of merit, see Chaps, V and VI...... 330 definition ........................ 257 values (Table 21, graphic, Figs. $5^{1-120}$ ) $372-376$,

Fin. See Cooling surface, indirect.

Fin and tube radiators (see also Types of

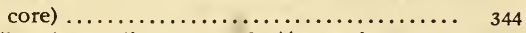
Flat plate radiators (see also Types of core).. 342-344

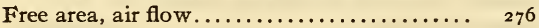
definition.......................... 254 head resistance . . . . . . . . . . . . . . 292

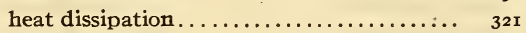
measurement................... 352 performance........................ $33 \mathrm{I}$

Frontal area, head resistance ........... 292

requirements at altitudes............. $35^{8}$

Fuselage, model................. 279, 296

Gage (see also Apparatus) . . . . . . . . . . 259 Geometrical characteristics (Chap. V)...... 330

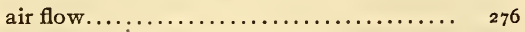
definitions..................... 253, 254 heat dissipation .................. 317 measurement...................... 350 performance (Chap. V) ............. 330 values (Table 20 ) ............... 363-37

Gregg, W. R.................. 253, 355

Head resistance (Chap. III) . . . . . . . . 28 I air flow.................... ${ }_{28} 8 \mathbf{r}$ at altitudes...................... 355, 357 apparatus..................... ${ }_{282}$ chargeable to radiator................ 294

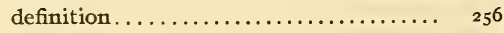
computation. ................... 284 cooling surface.................. 293, 332
Head resistance, definition. . . P.......

density of air.................. 291, 355 depth of core. ................. 293, 337 empirical equation................ 292 experimental data (Figs. $51-120$ ) . . . . 377-392 fuselage, model.................. 296 geometrical characteristics (Chap. V) . . 293, 330 measurement. See Measurements.

nose position . . . . . . . . . . . . . . . . . 296 obstructed positions............ 296-300, 353 power absorbed.................. 300 shuttering........................ 36 I slip stream. ................... 300 special types of radiators. . . . . . . . . . 343 speed....................... 291, 292, 300 stream-line casing. . . . . . . . . . . . . 348 surface friction. . . . . . . . . . . . . . ${ }_{28} 8$ temperature difference............ 290, $29 \mathrm{I}$ turbulence................... 25 I, 34 I unobstructed positions.......... 294, 352, 353 values (Table $2 \mathrm{r}$ ) ................. $372-376$

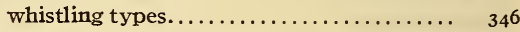
wing radiator....................... 299 yaw........................ 294, 354 Head resistance constant. . . . . . . . 255, 292 Head resistance factor . ........... 256, $28 \mathrm{r}, 343$

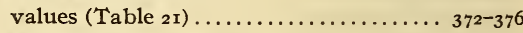

Heat dissipation (Chap. IV) ........... 303 air flow............................... 318 apparatus................. 303, 304, 308, 312

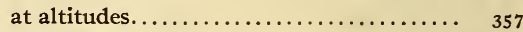
comparison of steam and water......... $3{ }_{34}$ computation.................... 306, 3 II cooling surface........ 303, 314, 315, 322, 323, 33 I

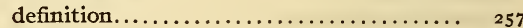
densiry of air................. 315,316, 355 depth of core................ 317,323, 333 empirical equations................ 317,356 experimental data. See Reduced pressure tunnel, Steam tunnel.

fin and tube radiators. . . . . . . . . . . . . 344 geometrical characteristics............ 339 indirect cooling surface. See Cooling surface, indirect.

measurement. See Measurements, heat dissipation.

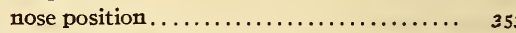

reduced pressure wind tunnel.......... 308

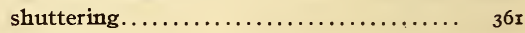
slip stream. ..................... 353 speed of flight . . . . . . . . . . . . . . . 315 steam tunnel................... 303, 3 I2 $_{2}$ stream-line casing. . . . . . . . . . . . . 348 temperature difference, in metal......... 314 water to air........... 253, 315,317, 319, 402 turbulence in air flow............... $257,34^{\circ}$ unobstructed positions............ 352, 353 values (Table 21 , graphic, Figs. $5 x-120$ ) .. 372$376,377-392$ water flow..................... 312, 31

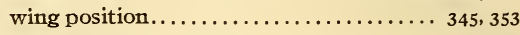
yaw.................................. 354 Heat dissipation coefficient........... 3I $7^{-32 I}$

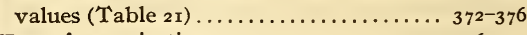
Heat of vaporization............... 306, 314 Heat transfer. See Heat dissipation. 
Hexagonal cell radiators. See Types of core.

Humidity ........................... 262-265

Hydraulic radius of air tubes, definition..... 255 equation for air flow.................. $\quad 276$ equation for heat dissipation........... 320, 322

Hydraulic radius of water tubes. ........ 255, 35 I

Hygrometer.................. 262, 266, 308, 3 I0

Indirect cooling surface. See Cooling surface, indirect.

Isothermal lines in air tubes. 398, 399

Lanchester, F. W.

Latent heat. See Heat of vaporization.

Lees, C. H........................... 287 equation for surface friction............. 287,320

Length of core.......................... $\quad 254$

Length of fin ........................ 404

Lift .......................... 299, 300, 302

Lift-drift ratio ....................... 302, 356

Long tubes, theory of................. 272, 286

Mass flow of air. See Air flow.

Mean temperature of steam and water.... 306,314

Mean temperature of water............. 3II, 3I4

Measurements. See Apparatus.

air flow, reduced pressure wind tunnel... 259, 264 steam tunnel...................... 259

air tubes.......................... 35 r, 352 cooling surface....................... 352

density of air...................... ${ }_{263}$ geometrical characteristics.............. 350 head resistance................ 282, 296, 299 heat dissipation, reduced pressure tunnel.. 308 steam tunnel..................... 303,312 pressure difference.................... $\quad 262$ temperature difference, reduced pressure

$$
\text { tunnel....... }
$$

steam tunnel.

temperature distribution in air tubes...... 397 temperature gradients in air tubes........ 397 water tubes........................... 350

Metal............................. 314, 330

Nose radiator.................... 279, 296, 353

Notation.......................... 393

Number of water tubes (Table 20).... 351, 363-37I

Nusselt, W....................... 394

Obstructed positions, air flow. . . . . . . . . 279-28r definition............................ 253 general performance................... 353 head resistance.................... 296, 300 heat dissipation. . . . . . . . . . . . . . . . . Oiled surface. . . . . . . . . . . . 288, 289, 326, 33 I Open wind tunnel, air flow measurements... 259 , $267-270,275$ head resistance measurements......... 282, 299

Perforated tube (whistling) radiators (see also Types of core) .................... 345 Performance of radiator (graphic, Figs. 51I20) ................... 332, 354, 377-392 Physical properties, definitions........... 255

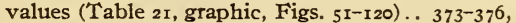
$377-392$

Piezometer rings 259,304

Pitot grid.......................... 259, 304 Pitot-static tubes. $267,268,282,348$
Pivoted radiators....................... 36

Polished surface, air flow................ 277

head resistance.................... 288, 293

heat transfer....................... 323

performance..................... 331, 332

Positions, definitions................... 253

performance.......................... 352-354

Power absorbed........................ 300

at altitudes.......................... 354

definition..............................

position.......................... 353

special types of core...................

values (graphic, Figs. 51-I20) ......... 377-392

Pressure difference, air flow............... 273 measurements........................ ${ }_{262}$

pressure tube........................ 268, 269

surface........................ 325

temperature difference................... 290

viscous or turbulent flow................ $\quad 258$

wing radiator......................... 280

Pressure gradient. See Pressure difference.

comparison of radiator tubes with long

tubes.

experimental data (Figs. $\left.13^{8}-152\right) \ldots \ldots 44^{12-426}$

single long tube ...................... $\quad 288$ surface................... 288, 289, 331, 332 Pressure tube (see also Apparatus) ........ 268, 269 comparison with air venturi meter....... 275 Propeller. See Slip-stream positions.

Pseudocellular radiators................ 339, 394

Rate of water flow. See Water flow.

Reduced pressure wind tunnel, air flow measurements.................. 259, 26

calorimetric measurements.............. 308 Resistance thermometer................ 264, 308 Retractable radiators...................... $36 \mathrm{I}$ Roughened surface.................. 288, 324 Rowse, W. C....................... ${ }_{26} 6_{3}$

Shape, effect on head resistance.......... 285,292 Shuttering. .......................... 349, 36r Side radiators ......................... $36 x$ Size, effect on head resistance........... 285, 292 Slip-stream positions, air flow ............ 280, 28 head resistance....................... 300 performance......................... 353 power absorbed....................... 303 Smoke stream ........................ 26r, 394 Smoked surface, heat transfer............. 326 performance......................... pressure gradient .................. 288,289

Source of heat for tests, hot water.. 303, 309, 312, 314 steam ........................ 303, 304, 307, 31 Special types of core (see also Types of core)... 341 Speed of flight, air flow............... 275, 276 definition.......................... 253 distribution, open wind tunnel........... 267 radiator tube........................ $\quad 270$ reduced pressure tunnel............... $\quad 264$ steam tunnel....................... 260 head resistance. ................. 291, 292,300 heat dissipation $\ldots \ldots \ldots \ldots \ldots \ldots \ldots \ldots \ldots, 250,395$ power absorbed ...................... 300 pressure differences..................... $\quad{ }_{273}$ slip-stream speed ..................... 280 Spiral vanes, head resistance............. 294 


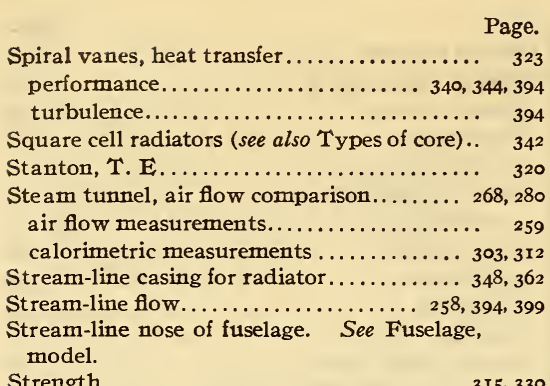

Strength............................ 3 I5, 330

Surface cooling coefficient. See Heat dissipation coefficient.

Surface friction ................. 257, 28r, 286

relation to heat dissipation............ 320

Surface transfer coefficient. See Heat dissipation coefficient.

Temperature, air................... ${ }_{26}$ at altitudes........................ 354 mean, steam and water............. 306, 307 water in radiators. . . . . . . . . . . . . . 314

Temperature difference, air and metal.... 327,404 air and water, at altitudes............ 355 definition $\ldots \ldots \ldots \ldots \ldots \ldots \ldots \ldots \ldots, 253,318,402$ heat dissipation.................. 315 pressure difference................ 290 air passage through radiator....... 3II, 3I2, 397 metal........................ 315,402 water...................... 309, 3 I I, 3 I2

Temperature distribution in air tubes..... 397

Temperature gradients. . . . . . . . . . . . . 397, 404

Temperature measurements. See Measurements.

Thermal conductivity. See Conductivity, thermal.

Thermal contact.................. 327 both sides of fins................. 328, 404 one side of fins.................. 328, 406

Thermocouples, air temperature difference... 308 calibration........................ 300 temperature distribution. . . ........... 397 water temperature difference........... 3 Io

Thickness, fins.................. 332, 404 water tubes....................... 343, 35 I water tube walls.................. 330, 402

Thomas meter................... 264, 288, 325

Turbulence, air flow.................. 257 evidence........................... 394 head resistance. ................. 287, 294 heat dissipation . . . . . . . . . . . . . . 303, 323 performance...................... 340

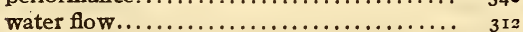

Turbulence, whistling radiators.......... ${ }_{346}$

Types of core....................... 34 I

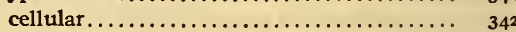

circular cell....................... 342

fin and tube............. 292, 294, $315,340,344$

flat plate...................... 342,343

hexagonal cell. .................. 340, 342

perforated plate types. . . . . . . . . . . . 294, 345

pseudocellular types................. 342

square cell....................... 342

whistling types. . . . . . $\ldots \ldots \ldots \ldots \ldots \ldots, 345$

Unobstructed positions, advantages ..... 352.353

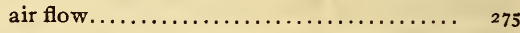

definition......................... 253

head resistance.................... 291, 294

performance of radiator.............. 352

Velocity distribution. See Speed, distribution.

Venturi meter, air............ 268, 274, 275, 279

water............................ 310

Viscosity of air................... 257, 320

Viscous flow.................. 258, 394, 399

Vulnerability.................. 251, 348

Water content of core............... 254,350

Water flow .................... 309, 310, 36 I effect on heat dissipation (graphic data, Figs.

I 28-137.............. 312-314, 36I, 409-4II

Water gage.................... 262, 270, 274

Water pump....................... 302, 310

Water tubes, definitions of dimensions....... 255

effects on performance............... 339

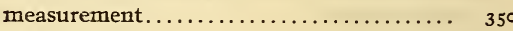

temperature drop in ................ $\quad 402$

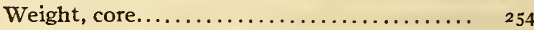
effect on power absorbed ........... 300, 302 effect on properties.............. 330 measurement.................. 352 steam condensed.................. 306 stream-line casing....................... 348 Whistling radiators (see also Types of core).. 345 Width of core................... 254, 348 Width of fin ................. 328, 332, 404 Wind tunnels, open tunnel. . . . . . 267, 282, 299 reduced pressure tunnel. . . . . . . . . . . . . ${ }_{264}$ steam tunnel. .................. 259, 260 54-inch. See Wind tunnels, open tunnel.

Window-blind shutters.............. $36 \mathrm{I}$

Wing position, air flow ................ 279

head resistance. . . . . . . . . . . . . . . . 299

performance...................... 345, 353

Yaw, air flow................... 278, 28 head resistance................. 284, 294, 299 performance...................... 354 